Universidade de São Paulo

Instituto de Astronomia, Geofísica e Ciências Atmosféricas

Departamento de Astronomia

Carla Martinez Canelo

\title{
O Mundo Aromático - dos PAHs no meio interestelar às condições bióticas
}

São Paulo 

Carla Martinez Canelo

\section{O Mundo Aromático - dos PAHs no meio interestelar às condições bióticas}

Dissertação apresentada ao Departamento de Astronomia do Instituto de Astronomia, Geofísica e Ciências Atmosféricas da Universidade de São Paulo como requisito parcial para a obtenção do título de Mestre em Ciências.

Área de Concentração: Astronomia Orientador(a): Prof. Dr. Amâncio César Santos Friaça

Versão Corrigida. O original encontra-se disponível na Unidade.

São Paulo 

Aos meus pais e minha irmã, que sempre me acompanharam e me apoiaram nesta jornada. A todos e todas que têm coragem de seguir seus sonhos. 



\section{Agradecimentos}

Ao meu pai Claudio por ter me dado a inspiração e a força, à minha mãe Silvana pela companhia e pelo suporte, e à minha irmã Carolina pela paciência e carinho. Vocês sempre serão a base do meu ser e da minha existência;

Ao Prof. Dr. Carlos Alexandre Wuensche de Souza por ter me mostrado o caminho para a Astrobiologia;

Ao Prof. Dr. Amâncio César Santos Friaça por me orientar desde a Iniciação Científica e por me apresentar a temas tão interessantes;

Aos pesquisadores Profa. Dra. Miriane G. Pastoriza, Dr. Daniel Ruschel-Dutra e, especialmente, Profa. Dra. Dinalva A. Sales, pela colaboração e por toda a ajuda e experiência;

Ao Prof. Dr. Amaury Almeida por ter acompanhado todo este trabalho e por sua atenção;

Aos colegas e amigos, Karin Fornazier, Miguel A. P. Murcia, Nathalia Cibirka, Patrícia de Novaes, Patricia da Silva, Roberto B. Menezese e Tiago Vecchi Ricci, pela ajuda com programação e com o dia-a-dia da pós-graduação;

A todo o pessoal da Secretaria e da Informática, por sempre estarem dispostos a fornecer assistência;

À CAPES e ao CNPq, pelo apoio financeiro;

Ao Instituto de Astronomia, Geofísica e Ciências Atmosféricas - IAG/USP.

Esta tese/dissertação foi escrita em ATEX com a classe IAGTESE, para teses e dissertações do IAG. 

"[...] humanity will reach maturity and wisdom on the day that it begins not just to tolerate, but take a special delight in differences in ideas and differences in life forms. [...] If we cannot learn to actually enjoy those small differences, to take a positive delight in those small differences between our own kind, here on this planet, then we do not deserve to go out into space and meet the diversity that is almost certainly out there."

Gene Roddenberry 



\section{Resumo}

Uma fração considerável do carbono no meio interestelar (ISM, da siga em inglês) $20 \%$ ou mais - está na forma de hidrocarbonetos policíclicos aromáticos (PAHs, na sigla em inglês), e as linhas de emissão do ISM no infravermelho médio são dominadas por bandas relacionadas a este tipo de moléculas (Joblin et al., 1992). Quando um PAH incorpora um ou mais átomos de nitrogênio, que substituem átomos de carbono, ele se torna um heterociclo policíclico aromático nitrogenado (PANH, da siga em inglês). Eles podem fornecer o elo perdido entre a química abundante dos PAHs no ISM e as nucleobases que compõem todos os seres vivos. A análise das características dos perfis de PAH, especialmente o de $6.2 \mu m$, poderia indicar a presença do nitrogênio incorporado aos anéis.

Peeters et al. (2002) sugeriu a divisão dos espectros de PAH em três classes - A, B e C

- dependendo da interpretação da variação da posição do pico dos perfis. A identificação da classe da banda de $6.2 \mu \mathrm{m}$ pode mostrar se PANHs estão presentes e quão importantes são para esta emissão. Neste trabalho, 206 galáxias (no geral, dominadas por starbursts), extraídas do projeto Spitzer/IRS ATLAS (Hernán-Caballero e Hatziminaoglou, 2011), tiveram seus perfis de $6.2 \mu m$ ajustados e distribuídos nas classes de Peeters.

Um total de 124 (60\%) galáxias foram classificadas como classe A, 42 (20\%) galáxias como classe B e 3 (1.5\%) galáxias como classe C. A classe A, correspondente a um comprimento de onda central perto de $6.22 \mu m$, só foi explicada pela substituição de carbono por nitrogênio, apesar de outras tentativas de explanação (Hudgins et al., 2005). Além disso, a classe B pode representar uma mistura entre PAHs e PANHs. Logo, estes espectros sugerem a presença significativa de PANHs, o que pode indicar outro reservatório de nitrogênio no Universo, com condições de densidade e temperatura diferentes das fases gasosas e gelos. 



\section{Abstract}

A considerable fraction of carbon in the interstellar medium - $20 \%$ or more - is in the form of polycyclic aromatic hydrocarbons (PAHs), and their mid infrared emission lines are dominated by bands related to this class of molecules (Joblin et al., 1992). When a PAH incorporates one or more atoms of nitrogen, that substitute the carbon atoms, it becomes a polycyclic aromatic nitrogen heterocycle (PANH). They can provide the missing link between the abundant PAHs chemistry at the ISM and the nucleobases that compose all living beings. Analyses of the PAHs features profiles, especially the $6.2 \mu \mathrm{m}$ feature, could indicate the presence of nitrogen incorporated to the rings.

Peeters et al. (2002) has suggested the division of PAH spectra into three classes - A, $\mathrm{B}$ and $\mathrm{C}$ - depending on the variation's interpretation of the profile peak positions. Identification of the feature class can show if PANHs are present and how important they are for this emission. For this work, 206 galaxies (starburst-dominated, in general), extracted from the Spitzer/IRS ATLAS project (Hernán-Caballero e Hatziminaoglou, 2011), have their 6.2 $\mu \mathrm{m}$ profiles fitted and distributed into the Peeter's classes.

A total of $124(60 \%)$ galaxies were classified as class A, 42 (20\%) galaxies as class $\mathrm{B}$ and $3(1.5 \%)$ galaxies as class $\mathrm{C}$. The class A, corresponding to a central wavelength near $6.22 \mu \mathrm{m}$, has only been explained by carbon replaced by nitrogen, despite other attempts of explanation (Hudgins et al. 2005). Besides, class B can represent a mix between PAHs and PANHs. Therefore, these spectra suggest a significant presence of PANHs, that could indicate another reservoir of nitrogen in the Universe, with density and temperature conditions different from those of gas phase and ices. 



\section{Lista de Figuras}

1.1 Exemplos de classificação da estrutura de PAHs (Imagem retirada de Andrews et al. (2015))

1.2 Evolução de moléculas carbonáceas e PAHs mediante radiação UV em am-

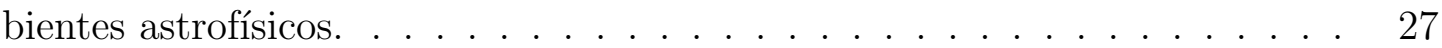

1.3 Diagrama da química interestelar do carbono top-down (Tielens, 2013). . . 28

1.4 Dois espectros são apresentados em densidade de fluxo versus comprimento de onda... (Yan et al., 2005) . . . . . . . . . . . . 30

1.5 Possíveis monômeros que poderiam ter constituído uma molécula genética primordial $($ Ehrenfreund et al. 2006) . . . . . . . . . . . . 31

1.6 Visão geral das possíveis variações no perfil das bandas (a) $3.3 \mu m$, (b) $6.2 \mu \mathrm{m}$, (c) $7.7 \mu \mathrm{m}$ e (d) $11.2 \mu \mathrm{m}$, divididas em três classes (A, B e C). . . . 34

1.7 Espectros calculados de três possíveis cátions de coroneno com substituição de $\mathrm{N}($ Hudgins et al., 2005). . . . . . . . . . . . 35

1.8 Distribuição interna do Spitzer . . . . . . . . . . . . . . . . . . . . . 37

1.9 Distribuição dos módulos do IRS. . . . . . . . . . . . . . . . . 37

1.10 Resumo das propriedades dos módulos do IRS . . . . . . . . . . . . . . . 38

2.1 Imagem BCD do objeto MIPS 289. . . . . . . . . . . . . 43

2.2 Teste de redução do background com o objeto MIPS 78. . . . . . . . . . . 4 43

2.3 Logotipo do SPICE . . . . . . . . . . . . . . . . . 45

2.4 Logotipo do SMART . . . . . . . . . . . . . . . . . . 45

2.5 Espectro da fonte MIPS289 extraído com o SMART. . . . . . . . . . . 46

3.1 Espectro do objeto 3C31 decomposto com o PAHFIT. . . . . . . . . 51 
3.2 Gráfico de RMS do objeto 3C31. . . . . . . . . . . . . . 51

3.3 Espectro do objeto $3 C 293$ decomposto com o PAHFIT. . . . . . . . . 552

3.4 Gráfico de RMS do objeto $3 C 293 \ldots \ldots \ldots$. . . . . . . . . . . . .

3.5 Espectro do objeto IRAS_01494-1845 decomposto com o PAHFIT. . . . . 52

3.6 Gráfico de RMS do objeto IRAS_01494-1845. . . . . . . . . . . . . . . 52

3.7 Espectro do objeto NGC7714 decomposto com o PAHFIT. . . . . . . 53

3.8 Gráfico de RMS do objeto NGC7714. . . . . . . . . . . . 53

3.9 Gráfico do valor parcial absoluto de RMS para as fontes por seus respectivos redshifts. ............................. 53

3.10 Gráfico do valor parcial normalizado de RMS para as fontes por seus respectivos redshifts.......................... 54

3.11 Perfil da banda de $6.2 \mu m$ do objeto 3C31. . . . . . . . . . 57

3.12 Perfil da banda de $6.2 \mu m$ do objeto GN26. . . . . . . . . . . 5 57

3.13 Perfil da banda de $6.2 \mu m$ do objeto MIPS8327. . . . . . . . . . 58

3.14 Perfil da banda de $6.2 \mu m$ do objeto MIPS22530 . . . . . . . . . . 58

3.15 Perfil da banda de $6.2 \mu m$ do objeto Arp220. . . . . . . . . . . 58

3.16 Perfil da banda de $6.2 \mu m$ do objeto NGC5033. . . . . . . . . . 58

3.17 Perfil da banda de $6.2 \mu m$ do objeto MIPS180. . . . . . . . . . . 59

3.18 Perfil da banda de $6.2 \mu m$ do objeto IC342. . . . . . . . . . . . . 59

3.19 Perfil da banda de $6.2 \mu m$ do objeto NGC2273. . . . . . . . . . 59

3.20 Perfil da banda de $6.2 \mu m$ do objeto NGC3227. . . . . . . . . . . . 60

3.21 Perfil da banda de $6.2 \mu m$ do objeto SST172458.3+591545 . . . . . . . . . 60

3.22 Perfil da banda de $6.2 \mu m$ do objeto EIRS-9. . . . . . . . . . . . 60

3.23 Perfil da banda de $6.2 \mu m$ do objeto EIRS-14. . . . . . . . . . . . 60

3.24 Perfil da banda de $6.2 \mu m$ do objeto MIPS289. . . . . . . . . . . . . 61

3.25 Perfil da banda de $6.2 \mu m$ do objeto MIPS15928. . . . . . . . . . . . . 61

3.26 Gráfico do comprimento de onda central da banda de $6.2 \mu m$ ajustado com o pah62_curvefit.py pelo redshift das fontes. . . . . . . . . . . . 6 62

3.27 Recorte da Figura 3.26 com valores de redshift $<0.25$. . . . . . . . . 62

3.28 Recorte da Figura 3.26 com valores de redshift $<0.035$. . . . . . . . . 62

3.29 Gráfico do comprimento de onda central da banda de $6.2 \mu m$ ajustado com o pah62_opt2.py pelo redshift das fontes. 
3.30 Recorte da Figura 3.29 com valores de redshift $(<0.25)$.

3.31 Gráfico do comprimento de onda central da banda de $6.2 \mu m$ pelo redshift das ULIRGs.

3.32 Recorte da Figura 3.31 com valores de redshift $<0.25$. . . . . . . . . . 65

3.33 Gráfico do comprimento de onda central da banda de $6.2 \mu \mathrm{m}$ pelo redshift das Seyferts.

3.34 Recorte da Figura 3.33 com valores de redshift $<0.04$.

3.35 Gráfico do comprimento de onda central da banda de $6.2 \mu \mathrm{m}$ pelo redshift dos Starbursts.

3.36 Recorte da Figura 3.35 com valores de redshift $<0.025 \ldots$. . . . . . . . 66

3.37 Gráfico do comprimento de onda central da banda de $6.2 \mu m$ pelo redshift de outros tipos de galáxias. . . . . . . . . . . . 67 67

3.38 Perfil da banda de $6.2 \mu m$ do objeto 3C293 ajustado com curve_fit. . . . . 68

3.39 Perfil da banda de $6.2 \mu m$ do objeto 3C293 ajustado com minimize. . . . . 68

3.40 Perfil da banda de 6.2 $\mu \mathrm{m}$ do objeto IRAS_00091-0738 ajustado com curve_fit. 68

3.41 Perfil da banda de 6.2 $\mu \mathrm{m}$ do objeto IRAS_00091-0738 ajustado com minimize. 68

3.42 Perfil da banda de $6.2 \mu m$ do objeto IRAS_11387+4116 ajustado com curve_fit. 69

3.43 Perfil da banda de $6.2 \mu m$ do objeto IRAS_11387+4116 ajustado com $m i$ -

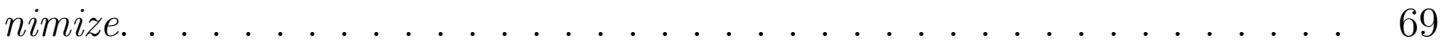

3.44 Perfil da banda de $6.2 \mu m$ do objeto IRAS_15225+2350 ajustado com curve_fit. 69

3.45 Perfil da banda de $6.2 \mu m$ do objeto IRAS_15225+2350 ajustado com mi-

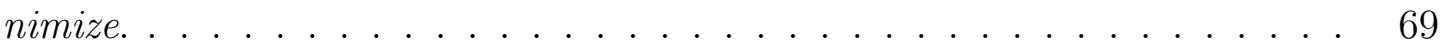

3.46 Perfil da banda de $6.2 \mu m$ do objeto MIPS16144 para $6.1 \leq \lambda \leq 6.27$.. . . 70

3.47 Perfil da banda de 6.3 $\mu m$ do objeto MIPS16144 para $6.26 \leq \lambda \leq 6.47$. . 70

3.48 Perfil da banda de $6.2 \mu m$ do objeto NGC7603. . . . . . . . . . . . . . . 70

3.49 Perfil da banda de $6.2 \mu m$ do objeto MIPS506. . . . . . . . . . . . . . 71

3.50 Perfil da banda de $6.2 \mu m$ do objeto MIPS22699. . . . . . . . . . . . . . 71

3.51 Perfil da banda de 6.2 $\mu m$ do objeto EIRS-37 ajustado com curve_fit. . . . 71

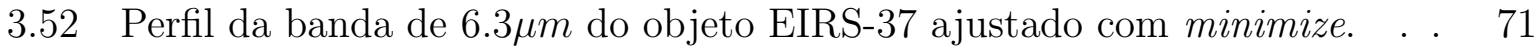

3.53 Perfil da banda de $6.2 \mu m$ do objeto IRAS_11028+3130 ajustado com curve_fit. 72

3.54 Perfil da banda de $6.3 \mu m$ do objeto IRAS_11028+3130 ajustado com $m i$ nimize. 
3.55 Perfil da banda de 6.2 $\mu m$ do objeto IRAS_11095-0238 ajustado com curve_fit. 72

3.56 Perfil da banda de 6.3 $\mathrm{m}$ do objeto IRAS_11095-0238 ajustado com minimize. 72

3.57 Perfil da banda de $6.2 \mu m$ do objeto MIPS16059 . . . . . . . . . . 73

3.58 Perfil da banda de $6.2 \mu m$ do objeto NGC3031. . . . . . . . . . . 73

3.59 Perfil da banda de $6.2 \mu m$ do objeto SJ103809.18+583226.2 . . . . . . 73

3.60 Banda de $6.0 \mu m$ presente no objeto EIRS-9. . . . . . . . . . . . 74

3.61 Banda de $6.0 \mu m$ presente no objeto IRAS03209-0806. . . . . . . . . . 74

3.62 Banda de $6.0 \mu m$ presente no objeto $\mathrm{M}+0-29-23 . \ldots \ldots$. . . . . 75

3.63 Banda de $6.0 \mu m$ presente no objeto Mrk938. . . . . . . . . . . . 75

4.1 Imagem representativa dos espectros das classes de Peeters, retirada de Tielens (2008), mostrando as distâncias entre cada banda que podem ser calculadas. . . . . . . . . . . . . . . . . . . . 78

4.2 Bandas ajustadas de PAHs do objeto 3C31. . . . . . . . . . 80

4.3 Bandas ajustadas de PAHs do objeto 3C293. . . . . . . . . . . 80

4.4 Espectro do objeto NGC4818 ajustado com o PAHTAT. . . . . . . . . . 81

B.1 Espectro do objeto Arp220 decomposto com o PAHFIT. . . . . . . . . 95

B.2 Gráfico de RMS do objeto Arp220. . . . . . . . . . . . . 95

B.3 Espectro do objeto EIRS-14 decomposto com o PAHFIT. . . . . . . . . 95

B.4 Gráfico de RMS do objeto EIRS-14. . . . . . . . . . . . . . 95

B.5 Espectro do objeto EIRS-37 decomposto com o PAHFIT. . . . . . . . . 96

B.6 Gráfico de RMS do objeto EIRS-37. . . . . . . . . . . . . . . . 96

B.7 Espectro do objeto EIRS-9 decomposto com o PAHFIT. . . . . . . . . . 96

B.8 Gráfico de RMS do objeto EIRS-9. . . . . . . . . . . . . . 96

B.9 Espectro do objeto GN26 decomposto com o PAHFIT. . . . . . . . . 96

B.10 Gráfico de RMS do objeto GN26. . . . . . . . . . . . . . . . 96

B.11 Espectro do objeto IC342 decomposto com o PAHFIT. . . . . . . . . . . 97

B.12 Gráfico de RMS do objeto IC342. . . . . . . . . . . . . . 97

B.13 Espectro do objeto IRAS03209-0806 decomposto com o PAHFIT. . . . . . 97

B.14 Gráfico de RMS do objeto IRAS03209-0806. . . . . . . . . . . . 97

B.15 Espectro do objeto IRAS_00091-0738 decomposto com o PAHFIT. . . . . 97

B.16 Gráfico de RMS do objeto IRAS_00091-0738. . . . . . . . . . . . . . 97 
B.17 Espectro do objeto IRAS_11028+3130 decomposto com o PAHFIT. . . . . 98

B.18 Gráfico de RMS do objeto IRAS_11028+3130. . . . . . . . . . . . . . . 98

B.19 Espectro do objeto IRAS_11095-0238 decomposto com o PAHFIT. . . . . 98

B.20 Gráfico de RMS do objeto IRAS_11095-0238. . . . . . . . . . . . . . . 98

B.21 Espectro do objeto IRAS_11387+4116 decomposto com o PAHFIT. . . . . 999

B.22 Gráfico de RMS do objeto IRAS_11387+4116. . . . . . . . . . . . . . . 99

B.23 Espectro do objeto IRAS_15225+2350 decomposto com o PAHFIT. . . . . 99

B.24 Gráfico de RMS do objeto IRAS_15225+2350. . . . . . . . . . . . . . . 99

B.25 Espectro do objeto M+0-29-23 decomposto com o PAHFIT. . . . . . . . . 99

B.26 Gráfico de RMS do objeto M+0-29-23. . . . . . . . . . . . . . . 999

B.27 Espectro do objeto MIPS15928 decomposto com o PAHFIT. . . . . . . . 100

B.28 Gráfico de RMS do objeto MIPS15928. . . . . . . . . . . . . . . . . . 100

B.29 Espectro do objeto MIPS16059 decomposto com o PAHFIT. . . . . . . . 100

B.30 Gráfico de RMS do objeto MIPS16059. . . . . . . . . . . . . . . 100

B.31 Espectro do objeto MIPS16144 decomposto com o PAHFIT. . . . . . . . 100

B.32 Gráfico de RMS do objeto MIPS16144. . . . . . . . . . . . . . . . . . . 100

B.33 Espectro do objeto MIPS180 decomposto com o PAHFIT. . . . . . . . . . 101

B.34 Gráfico de RMS do objeto MIPS180. . . . . . . . . . . . . . . . . 101

B.35 Espectro do objeto MIPS22530 decomposto com o PAHFIT. . . . . . . . 101

B.36 Gráfico de RMS do objeto MIPS22530. . . . . . . . . . . . . . . . 101

B.37 Espectro do objeto MIPS22699 decomposto com o PAHFIT. . . . . . . . 101

B.38 Gráfico de RMS do objeto MIPS22699. . . . . . . . . . . . . . . . . . . 101

B.39 Espectro do objeto MIPS289 decomposto com o PAHFIT. . . . . . . . . . 102

B.40 Gráfico de RMS do objeto MIPS289. . . . . . . . . . . . . . . 102

B.41 Espectro do objeto MIPS506 decomposto com o PAHFIT. . . . . . . . . . 102

B.42 Gráfico de RMS do objeto MIPS506. . . . . . . . . . . . . . . . . . 102

B.43 Espectro do objeto MIPS8327 decomposto com o PAHFIT. . . . . . . . . 102

B.44 Gráfico de RMS do objeto MIPS8327. . . . . . . . . . . . . . . . . 102

B.45 Espectro do objeto Mrk938 decomposto com o PAHFIT. . . . . . . . . . . 103

B.46 Gráfico de RMS do objeto Mrk938. . . . . . . . . . . . . . 103

B.47 Espectro do objeto NGC2273 decomposto com o PAHFIT. . . . . . . . 103

B.48 Gráfico de RMS do objeto NGC2273. . . . . . . . . . . . . . . . . . 103 
B.49 Espectro do objeto NGC3031 decomposto com o PAHFIT. . . . . . . . 103

B.50 Gráfico de RMS do objeto NGC3031. . . . . . . . . . . . . . . 103

B.51 Espectro do objeto NGC3227 decomposto com o PAHFIT. . . . . . . . . 104

B.52 Gráfico de RMS do objeto NGC3227. . . . . . . . . . . . . . . . . . . . 104

B.53 Espectro do objeto NGC5033 decomposto com o PAHFIT. . . . . . . . 104

B.54 Gráfico de RMS do objeto NGC5033. . . . . . . . . . . . . . . 104

B.55 Espectro do objeto SJ103809.18+583226.2 decomposto com o PAHFIT. . 105

B.56 Gráfico de RMS do objeto SJ103809.18+583226.2. . . . . . . . . . . . 105

B.57 Espectro do objeto SST172458.3+591545 decomposto com o PAHFIT. . . 105

B.58 Gráfico de RMS do objeto SST172458.3+591545 . . . . . . . . . . . . 105

C.1 Perfil da banda de $6.2 \mu m$ do objeto EIRS-2. . . . . . . . . . . . 107

C.2 Perfil da banda de $6.2 \mu m$ do objeto EIRS-13. . . . . . . . . . . . . 107

C.3 Perfil da banda de $6.2 \mu m$ do objeto IRAS_23129+2548. . . . . . . . . . . 108

C.4 Perfil da banda de $6.2 \mu m$ do objeto LH_H901A. . . . . . . . . . . . . . . 108

C.5 Perfil da banda de $6.2 \mu m$ do objeto MIPS22432. . . . . . . . . . . . . 108

C.6 Perfil da banda de $6.2 \mu m$ do objeto MIPS8242. . . . . . . . . . . . . . 108

C.7 Perfil da banda de $6.2 \mu m$ do objeto Mrk938. . . . . . . . . . . . . . . 109

C.8 Perfil da banda de $6.2 \mu m$ do objeto Murphy22. . . . . . . . . . . . . 109

C.9 Perfil da banda de $6.2 \mu m$ do objeto Murphy3. . . . . . . . . . . . . . 109

C.10 Perfil da banda de $6.2 \mu m$ do objeto Murphy8. . . . . . . . . . . . . . . 109

C.11 Perfil da banda de $6.2 \mu m$ do objeto NGC1056. . . . . . . . . . . . . 109

C.12 Perfil da banda de $6.2 \mu m$ do objeto NGC1097. . . . . . . . . . . . . . 109

C.13 Perfil da banda de $6.2 \mu m$ do objeto NGC1365. . . . . . . . . . . . . . . 110

C.14 Perfil da banda de $6.2 \mu m$ do objeto NGC1566. . . . . . . . . . . . . . 110

C.15 Perfil da banda de $6.2 \mu m$ do objeto NGC3256. . . . . . . . . . . . . 110

C.16 Perfil da banda de $6.2 \mu m$ do objeto NGC3511. . . . . . . . . . . . . . . 110

C.17 Perfil da banda de $6.2 \mu m$ do objeto NGC5135. . . . . . . . . . . . . 111

C.18 Perfil da banda de $6.2 \mu m$ do objeto NGC5953. . . . . . . . . . . . . . 111

C.19 Perfil da banda de $6.2 \mu m$ do objeto NGC7469. . . . . . . . . . . . . 111

C.20 Perfil da banda de $6.2 \mu m$ do objeto NGC7582. . . . . . . . . . . . . . 111

C.21 Perfil da banda de 6.2 $\mu m$ do objeto SWIRE4_J104057.84+565238.9 . . . 112 


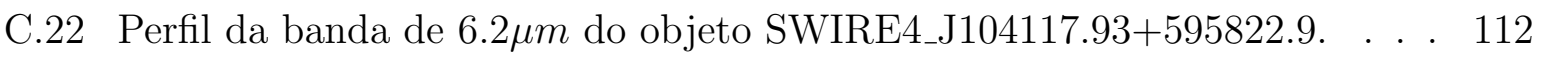

C.23 Perfil da banda de $6.2 \mu m$ do objeto AGN15. . . . . . . . . . . . . . 113

C.24 Perfil da banda de $6.2 \mu m$ do objeto E12-G21 . . . . . . . . . . . . 113

C.25 Perfil da banda de $6.2 \mu m$ do objeto IRAS14121-0126. . . . . . . . . . . . 113

C.26 Perfil da banda de $6.2 \mu m$ do objeto IRAS14197+0813. . . . . . . . . . . . 113

C.27 Perfil da banda de $6.2 \mu m$ do objeto IRAS14202+2615 . . . . . . . . . . 114

C.28 Perfil da banda de $6.2 \mu m$ do objeto IRAS14485-2434. . . . . . . . . . . . 114

C.29 Perfil da banda de $6.2 \mu m$ do objeto IRAS_02411+0353. . . . . . . . . . . 114

C.30 Perfil da banda de $6.2 \mu m$ do objeto IRAS_03250+1606. . . . . . . . . . . 114

C.31 Perfil da banda de $6.2 \mu m$ do objeto IRAS_13539+2920 . . . . . . . . . . . 114

C.32 Perfil da banda de $6.2 \mu m$ do objeto IRAS_14060+2919. . . . . . . . . . 114

C.33 Perfil da banda de $6.2 \mu m$ do objeto IRAS_14252-1550 . . . . . . . . . . 115

C.34 Perfil da banda de $6.2 \mu m$ do objeto IRAS_14348-1447. . . . . . . . . . . 115

C.35 Perfil da banda de $6.2 \mu m$ do objeto IRAS_17028+5817. . . . . . . . . . . 115

C.36 Perfil da banda de $6.2 \mu m$ do objeto IRAS_17068+4027. . . . . . . . . . . 115

C.37 Perfil da banda de $6.2 \mu m$ do objeto IRAS_20414-1651. . . . . . . . . . . 116

C.38 Perfil da banda de $6.2 \mu m$ do objeto IRAS_21208-0519 . . . . . . . . . . 116

C.39 Perfil da banda de $6.2 \mu m$ do objeto MIPS8342 . . . . . . . . . . . 116

C.40 Perfil da banda de $6.2 \mu m$ do objeto MIPS8493. . . . . . . . . . . . 116

C.41 Perfil da banda de $6.2 \mu m$ do objeto MIPS22277. . . . . . . . . . . . . 117

C.42 Perfil da banda de $6.2 \mu m$ do objeto MIPS22482 . . . . . . . . . . . 117

C.43 Perfil da banda de $6.2 \mu m$ do objeto MIPS22651. . . . . . . . . . . . . 117

C.44 Perfil da banda de $6.2 \mu m$ do objeto Mrk273. . . . . . . . . . . . . 117

C.45 Perfil da banda de $6.2 \mu m$ do objeto NGC1222. . . . . . . . . . . . 118

C.46 Perfil da banda de $6.2 \mu m$ do objeto NGC1614. . . . . . . . . . . . . . . 118

C.47 Perfil da banda de $6.2 \mu m$ do objeto NGC3079. . . . . . . . . . . . . 118

C.48 Perfil da banda de $6.2 \mu m$ do objeto NGC3310 . . . . . . . . . . . . 118

C.49 Perfil da banda de $6.2 \mu m$ do objeto NGC3556. . . . . . . . . . . . . . 119

C.50 Perfil da banda de $6.2 \mu m$ do objeto NGC4088. . . . . . . . . . . . . 119

C.51 Perfil da banda de $6.2 \mu m$ do objeto NGC4194. . . . . . . . . . . . . 119

C.52 Perfil da banda de $6.2 \mu m$ do objeto NGC4676. . . . . . . . . . . . . . 119

C.53 Perfil da banda de $6.2 \mu m$ do objeto NGC5194. . . . . . . . . . . . . . . 120 
C.54 Perfil da banda de $6.2 \mu m$ do objeto NGC5256. . . . . . . . . . . 120

C.55 Perfil da banda de $6.2 \mu m$ do objeto CGCG381-051. . . . . . . . . 121

C.56 Perfil da banda de $6.2 \mu m$ do objeto EIRS-55 . . . . . . . . . . . 121

C.57 Perfil da banda de $6.2 \mu m$ do objeto GN39a. . . . . . . . . . . . 121

C.58 Perfil da banda de $6.2 \mu m$ do objeto GN39b. . . . . . . . . . . . . 121

C.59 Perfil da banda de $6.2 \mu m$ do objeto IRAS08474+1813 . . . . . . . . . 122

C.60 Perfil da banda de $6.2 \mu m$ do objeto IRAS_00482-2721. . . . . . . . . . . 122

C.61 Perfil da banda de $6.2 \mu m$ do objeto IRAS_01298-0744 ajustado com curve_fit.122

C.62 Perfil da banda de 6.2 $\mu m$ do objeto IRAS_01298-0744 ajustado com minimize.122

C.63 Perfil da banda de 6.2 $\mu \mathrm{m}$ do objeto IRAS_01569-2939 ajustado com curve_fit.122

C.64 Perfil da banda de 6.2 $\mu m$ do objeto IRAS_01569-2939 ajustado com minimize.122

C.65 Perfil da banda de $6.2 \mu m$ do objeto IRAS_10035+2740 ajustado com curve_fit.123

C.66 Perfil da banda de $6.2 \mu m$ do objeto IRAS_10035+2740 ajustado com minimize. . . . . . . . . . . . . . . . . . . . 123

C.67 Perfil da banda de $6.2 \mu m$ do objeto MIPS8521 . . . . . . . . . . . 123

C.68 Perfil da banda de $6.2 \mu m$ do objeto NGC1241 . . . . . . . . . . . . 123

C.69 Perfil da banda de $6.2 \mu m$ do objeto NGC4594. . . . . . . . . . . . 123

C.70 Perfil da banda de $6.2 \mu m$ do objeto NGC5929 . . . . . . . . . . . 123 


\section{Lista de Tabelas}

1.1 Modos de vibração de algumas bandas de PAHs. . . . . . . . . . . . . . 33

2.1 Nomes e redshifts das ULIRGs utilizadas por Yan et al. (2007). . . . . . . 41

3.1 Cores utilizadas nos gráficos com os ajustes do PAHFIT e as componentes do modelo à que se atribuem. . . . . . . . . . . . . . . . 50

3.2 Intervalo para cada classe de Peeter. . . . . . . . . . . . . 56

3.3 Quantidade de galáxias categorizadas em cada classe de Peeters. . . . . . . 61

3.4 Distribuição das classes de Peeters de acordo com o tipo de galáxia. . . . . 64

4.1 Palpites iniciais para o ajuste das bandas de PAHs. . . . . . . . . . 79

D.1 Fontes e suas respectivas informações retiradas do catálogo do projeto Spit$z e r / I R S$ ATLAS. . . . . . . . . . . . . . . . 126

D.2 Resultados parcias e totais de RMS. . . . . . . . . . . . . 135

D.3 Resultados do ajuste da banda de $6.2 \mu m$ com o pah62_curvefit.py. . . . . . 141

D.4 Resultados do ajuste da banda de $6.2 \mu m$ com o pah62_opt2.py. . . . . . . . 149

D.5 Resultados das galáxias que apresentaram os melhores ajustes. . . . . . . 157 



\section{Sumário}

1. Introdução . . . . . . . . . . . . . . . . . . . . 25

1.1 PAHs no meio interestelar . . . . . . . . . . . . . 25

1.2 PAHs e objetos extragalácticos . . . . . . . . . . . . . 28

1.3 O Mundo Aromático e os PANHs . . . . . . . . . . . . . . . . . . . 30

1.4 Observando PAHs e PANHs . . . . . . . . . . . . . . . . . . . 32

1.4.1 As Classes de Peeters . . . . . . . . . . . . . . . . . . 32

1.4.2 Spitzer Space Telescope e o IRS . . . . . . . . . . . . . 36

1.5 Visão geral dos objetivos . . . . . . . . . . . . . . . . . . . . . . . . . . . . 39

1.6 Organização da Dissertação . . . . . . . . . . . . . . . . . . . . 39

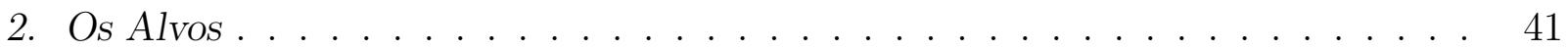

2.1 Primeiras opções . . . . . . . . . . . . . . . . . . 41

2.1.1 Redução de dados . . . . . . . . . . . . . . . . . . . . . . . . 42

2.1.1.1 Background .................. 42

2.1.1.2 Rogue pixels ................. . . . 44

2.1.1.3 Coad ....................... 44

2.1.1.4 Extração espectral . . . . . . . . . . . . . . . 44

2.1.2 Considerações . . . . . . . . . . . . . . . . . . 45

2.2 O projeto Spitzer $/ I R S$ Atlas . . . . . . . . . . . . . . 46

$2.2 .1 \quad$ A amostra MIR starbursts . . . . . . . . . . . . 47

3. Análise de dados e resultados . . . . . . . . . . . . . . . . . . . . . . 49

3.1 PAHFIT . . . . . . . . . . . . . . . . . . 49

3.1 .1 RMS ......................... 50 
3.1 .2 Resultados . . . . . . . . . . . . . . . . . . 51

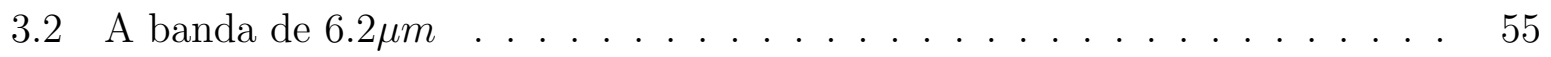

3.2 .1 Resultados . . . . . . . . . . . . . . . . . . 57

3.2.1.1 Melhor ajuste pelo pah62_curvefit.py _ . . . . . . 57

3.2.1.2 Melhor ajuste pelo pah62_opt2.py . . . . . . . . . . 59

3.2.1.3 Visão geral dos resutados . . . . . . . . . . . . . 61

3.2.1.4 Espectros peculiares . . . . . . . . . . . . 67 67

3.2.1.5 Ajustes inconclusivos . . . . . . . . . . . . . . 71

3.3 A banda de $6.0 \mu m \ldots \ldots \ldots \ldots \ldots \ldots \ldots \ldots$

4. Conclusões e perspectivas . . . . . . . . . . . . . . . . . . 77

4.1 Novas abordagens . . . . . . . . . . . . . . . . . . . . 78

4.2 Outros ambientes astrofísicos e novas observações . . . . . . . . . . . 81

Referências ....................... 83

Apêndice 91

A. Lista de abreviações . . . . . . . . . . . . . . . . . . . . . . 93

B. Espectros decompostos pelo PAHFIT e seus respectivos RMS . . . . . . 95

C. Perfis da banda de $6.2 \mu m$. . . . . . . . . . . . . . . 107

C.1 Melhor ajuste com o pah62_curvefit.py . . . . . . . . . . . . 107

C.2 Melhor ajuste com o pah62_opt2.py . . . . . . . . . . . . . . 113

C.3 Ajustes inconclusivos . . . . . . . . . . . . . . . . . 121

D. Fontes: identificação e propriedades derivadas . . . . . . . . . . . . 125 
Capítulo 1

\section{Introdução}

\subsection{PAHs no meio interestelar}

Uma fração considerável do carbono no meio interestelar (20\% ou mais) encontra-se na forma de PAHs (sigla em inglês para hidrocarbonetos policíclicos aromáticos), e a emissão em bandas no infravermelho médio é dominada pelas bandas desta classe de moléculas (Joblin et al., 1992), conhecida como AIBs (sigla em inglês para Bandas Aromáticas no Infravermelho). PAHs, basicamente, são diversos anéis aromáticos unidos, formados por átomos de carbono. Eles podem apresentar diversos tipos de estruturas (Figura 1.1), sendo os PAHs pericondensados e os simétricos os mais estáveis do ponto de vista da reatividade química e da fragmentação (Andrews et al., 2015).

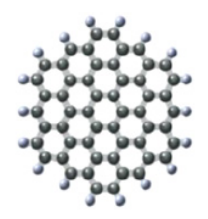

$\mathrm{C}_{54} \mathrm{H}_{18}$

a) Compact Pericondensed

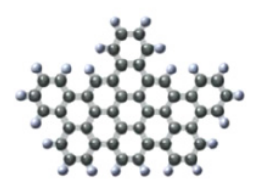

$\mathrm{C}_{48} \mathrm{H}_{22}$

e) Irregular

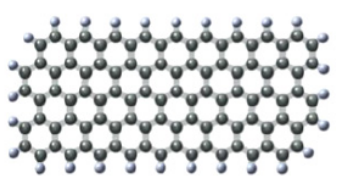

$\mathrm{C}_{98} \mathrm{H}_{28}$

b) Edged Pericondensed

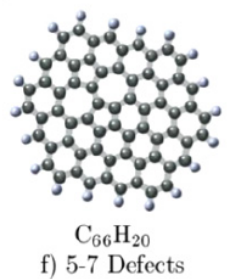

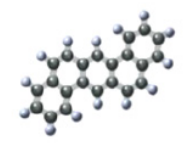

$\mathrm{C}_{22} \mathrm{H}_{14}$

c) Not Branched Catacondensed

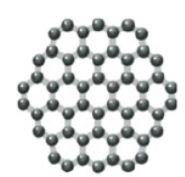

$\mathrm{C}_{54}$

g) Completely Dehydrogenated

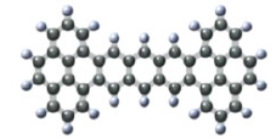

$\mathrm{C}_{42} \mathrm{H}_{22}$

d) Branched Catacondensed

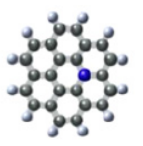

$\mathrm{C}_{23} \mathrm{H}_{12} \mathrm{~N}$

h) PANH

Figura 1.1: Exemplos de classificação da estrutura de PAHs. (a) e (b) - PAHs pericondensados: núcleos compactos de carbono. (c) e (d) - PAHs catacondensados: PAHs linares. (e) - PAHs cuja forma não se assemelha às demais classificações. (f) - PAHs com 5 a 7 defeitos de anéis fundidos. (g) - PAHs completamente desidrogenados: todos os hidrogênios foram removidos. (h) - PAHs nitrogenados: o nitrogênio substituindo um átomo de carbono do núcleo do PAH. (Imagem retirada de Andrews et al. (2015)) 
Até $50 \%$ da luminosidade emitida no infravermelho médio pode ser devida às bandas dos PAHs, as mais fortes estando a 3.3, 6.2, 7.7, 8.6, 11.3 e $12.7 \mu m$ (Li, 2004), e as mais

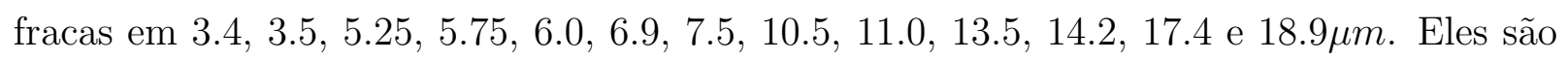
abundantes e onipresentes no meio interestelar das galáxias, incluindo quase todos os objetos astrofísicos correlacionados com poeira e gás e com iluminação por fótons ultravioleta (UV), como regiões HII, nebulosas de reflexão, estrelas jovens e AGBs (ramo assimptótico das gigantes), superfícies de nuvens escuras, entre outros (Tielens, 2008). Particularmente, PAHs dominam o aquecimento de gás neutro e o balanço de ionização em nuvens moleculares e são importantes traçadores de regiões de formação estelar.

Normalmente essas emissões são atribuídas à fluorescência de PAHs bombeados por fótons do UV longínquo, de cerca de 50 átomos de carbono. Isto porque as espécies menores de PAHs possuem uma baixa capacidade de calor e, ao aborverem um único fóton, elas ficam muito quentes, vibram e emitem no infravermelho médio (mid-IR), mesmo se estiverem distante da estrela. E, o perfil de muitas destas bandas é típico da anarmonicidade associada a emissões moleculares (Tielens, 2008). Estas emissões não dependem da intensidade do campo de radiação, e sim somente da energia dos fótons e das propriedades das partículas (Guimarães, 2006).

O principal parâmetro de um PAH para se calcular o quanto ele absorve de energia do campo de radiação, a que temperatura chega e como ocorre a reemissão, é a eficiência de absorção. Ela varia com a temperatura do grão e os dois modelos mais usados para seu cálculo são descritos em Dwek et al. (1997) e Li e Draine (2001). Já a capacidade térmica está intimamente relacionada com a configuração espacial dos anéis de carbono do PAH (Guimarães, 2006).

Acredita-se que PAHs e macromoléculas carbonáceas exercem um papel fundamental na física e química do meio interestelar (ISM, na sigla em ingês) (Berné et al., 2015). A Figura 1.2 apresenta um diagrama da evolução destas moléculas em diferentes ambientes astrofísicos. Em nuvens densas, grãos de poeira conseguem bloquear boa parte da radiação UV de estrelas próximas e há uma maior complexidade molecular. Contudo, a partir das bordas da nuvem, o campo de radiação começa a atuar cada vez mais e os grãos são processados e fragmentados em outras espécies moleculares menores.

Em relação à sua formação, processos de condensação de fuligem em material ejetado de estrelas podem criar grandes moléculas de PAHs. Os radicais iniciais são gerados 


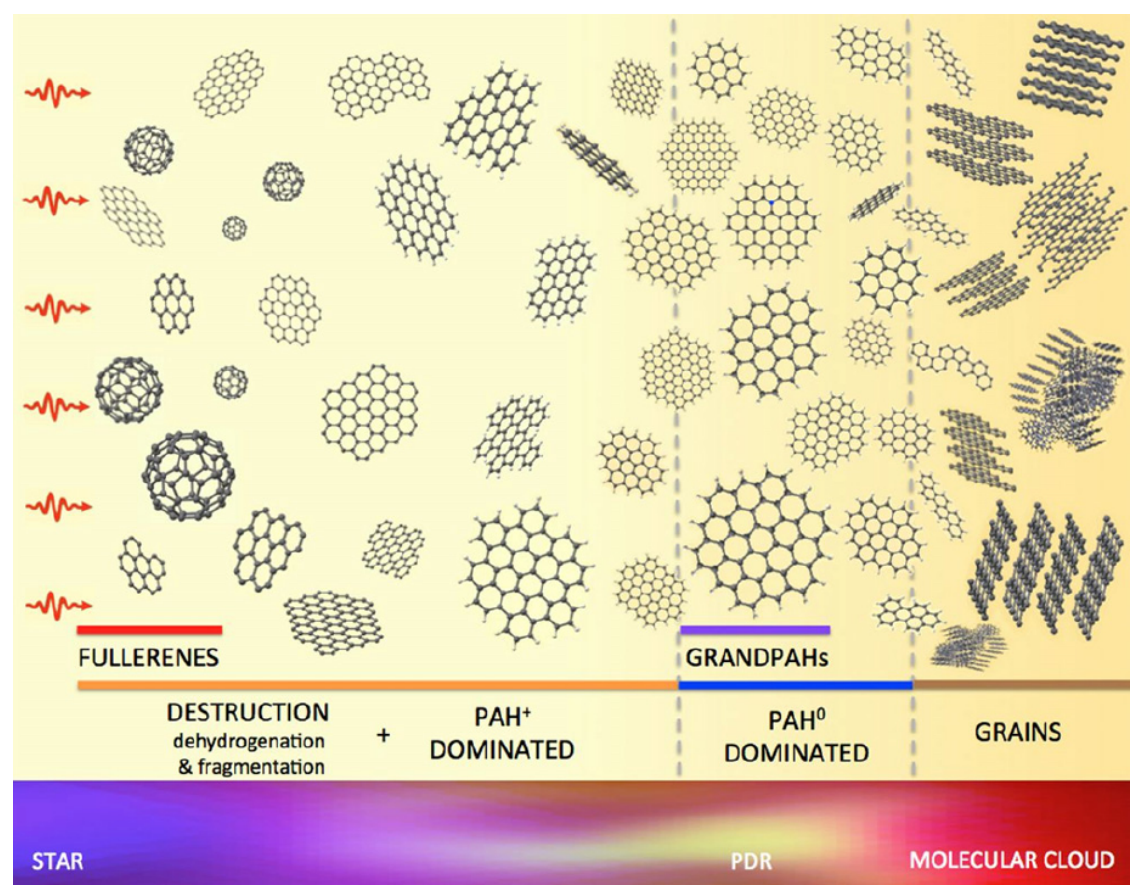

Figura 1.2: Evolução proposta de moléculas carbonáceas e PAHs mediante radiação UV em ambientes astrofísicos. (Figura retirada de Andrews et al. (2015)). No limite entre a nuvem molecular e a PDR (sigla em inglês para região de fotodissociação), PAHs são altamente processados e formam a população grandPAH, com as estruturas mais estáveis (pericondensados). Conforme maior a proximidade da estrela, mais os PAHs são ionizados e destruídos, gerando outras moléculas (por exemplo, o fulereno).

a partir de reações simples com o acetileno $\left(C_{2} H_{2}\right)$ e depois, com rápida adição de hidrogênio e reações de abstração, o fenil $\left(C_{6} H_{5}\right)$ estabelece um curso estável para o benzeno $\left(\mathrm{C}_{6} \mathrm{H}_{6}\right)$. Repetindo-se o mesmo processo (conhecido como HACA - Hydrogen-Abstraction aCetylene-Addition), anéis aromáticos passam a se adicionar. O ISM é enriquecido conforme PAHs se aglomeram em grandes estruturas moleculares e em grãos de poeira (Tielens, 2013). Por outro lado, no caso de nuvens moleculares, estas rotas químicas de íon-molécula (por exemplo, Equações 1.1 e 1.2) são mais consideradas para o crescimento de PAHs já existentes no ISM. Elas podem abranger diversas reações rápidas entre radicais de PAH e hidrocarbonetos como, por exemplo, a interação entre o radical fenil e o vinil acetileno $\left(\mathrm{CH}_{2}=\mathrm{CH}-\mathrm{C} \equiv \mathrm{CH}\right)$ que resulta no naftaleno (dois anéis benzênicos) (Tielens, 2013).

$$
\begin{aligned}
& P A H+X^{+} \rightarrow P A H^{+}+X \\
& P A H^{-}+X^{+} \rightarrow P A H+X
\end{aligned}
$$

Estes são exemplos da abordagem bottom-up, na qual pequenas moléculas reagem para 
formar espécies cada vez mais complexas. Seu oposto é a top-down, em que macromoléculas vão sendo quebradas em menores, como no caso da produção do fulereno $\left(C_{60}\right)$ a partir de PAHs (Berné et al., 2015). Esta última é muito utilizada quando se trata da fotoquímica de PAHs no ISM (Equação 1.3, onde R é um grupo lateral mas também poderia ser um H (Tielens, 2005)). A evolução química de PAHs no ISM se dá pela presença de fótons UV e pela combinação de efeitos de desidrogenação e fragmentação com os de isomerização, como esquematizado na Figura 1.3 (Tielens, 2013). Afinal, as densidades moleculares predominantes nestas regiões são ordens de magnitudes menores para um cenário bottomup.

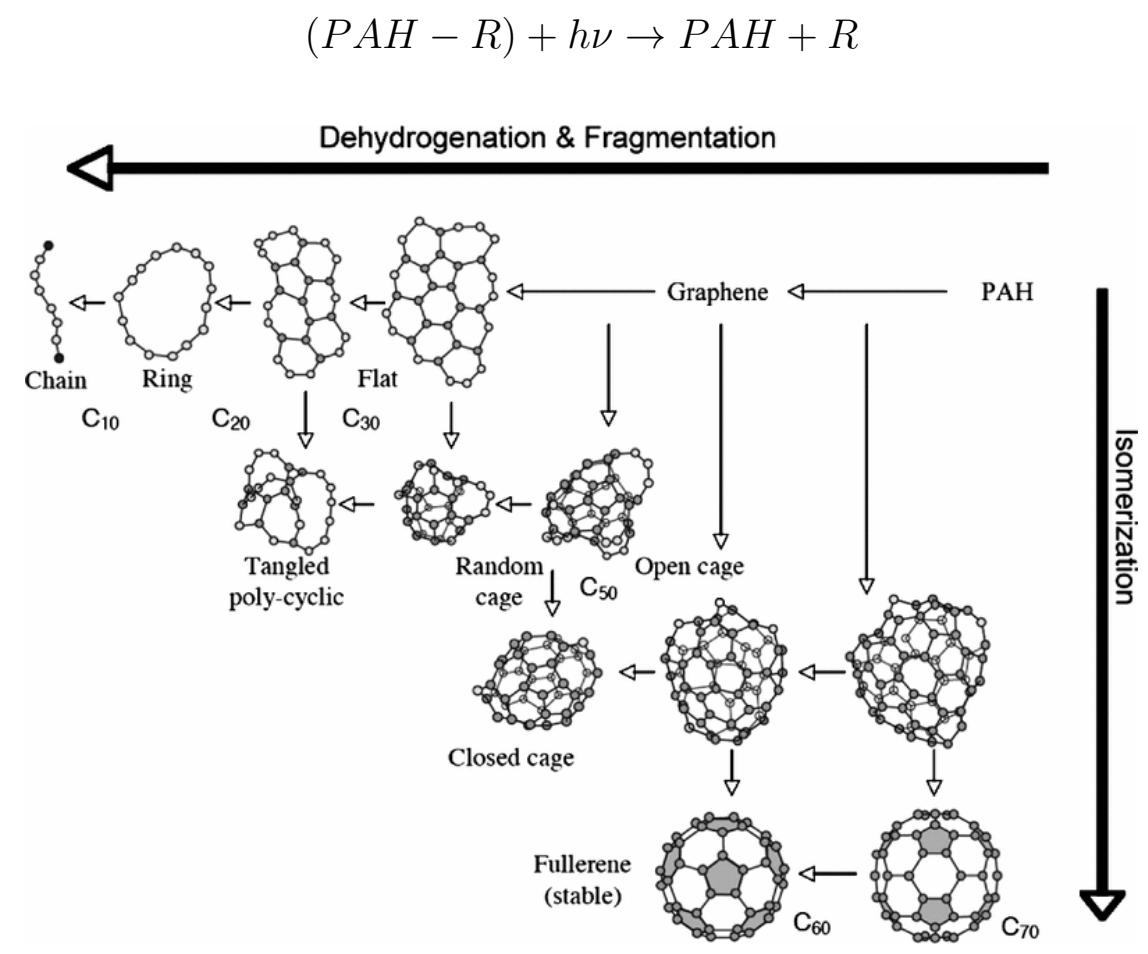

Figura 1.3: Diagrama da química interestelar do carbono top-down, extraído de Tielens (2013)).

\subsection{PAHs e objetos extragalácticos}

A alta luminosidade das bandas de PAHs permitem que estas sejam visíveis em objetos a alto redshift, onde dominariam o espectro no infravermelho (IR) (Papovich et al., 2006; Teplitz et al., 2007). Até recentemente, o redshift mais elevado para o qual bandas dos PAHs foram detectadas era de $z=3.074$ para a galáxia Cosmic Eye, uma Lyman Break Galaxy com forte lente gravitacional (Siana et al., 2009). Lyman Break Galaxies são 
galáxias com formação estelar intensa a altos redshifts. Anos depois, a banda de $6.2 \mu m$ foi observada na galáxia submilimétrica GN20, com $z=4.055$ (Riechers et al., 2014).

Inclusive, dados de arquivo do instrumento IRS (Houck et al., 2004) a bordo do telescópio Spitzer (Werner et al., 2004), cuja faixa espectral concentra as mais fortes bandas de emissão dos PAHs, já foram usados para determinar os redshifts de objetos a partir das bandas de PAHs identificadas. Em Yan et al. (2005, 2007), foram escolhidos diversos alvos, compreendendo valores de redshifts de 0.61 a 3.2, para os quais os respectivos espectros foram basicamente reduzidos a partir do IRS pipeline do Spitzer Science Center (SSC), submetidos a novos processamentos pelos autores e extraídos através do software SPICE (Spitzer IRS Custom Extraction) do SSC. As bandas mais utilizadas para o cálculo dos redshits foram as de 6.2, 7.7, 8.6, 9.8, 11.3 e $12.8 \mu \mathrm{m}$, cuja banda de $9.8 \mu \mathrm{m}$ se refere à absorção do $\mathrm{SiO}$ e as demais são emissões de PAHs.

Dois dos espectros obtidos por Yan et al. (2005), juntamente com o redshift extrapolado pelas bandas de PAHs, encontram-se na Figura 1.4. Análises sugerem que ambos sejam ULIRGs (Ultra Luminous Infra-Red Galaxies). ULIRGs possuem as luminosidades infravermelhas mais intensas. Uma hipótese é que elas representariam uma etapa da junção de duas galáxias espirais ricas em gás. Quando este gás molecular direciona-se para o núcleo, ele acarreta num surto de formação estelar e, em alguns casos, atividade AGN (sigla em inglês para núcleo galáctico ativo). Assim, a poeira absorve a radiação das estrelas jovens e/ou do AGN e a re-irradia (Guimarães, 2006).

Outro tipo de objeto com muitas emissões de PAHs é o starburst. Eles são regiões de galáxias com altas densidades de energia oriundas de ventos de estrelas massivas e explosões de supernova, que criam ondas de choque e outflows de gás no meio interestelar. Embora as ondas de choque derivadas de supernova destruam grande parte dos grãos de poeira, os outflows podem ejetar quantidades significativas de gás e poeira para fora das regiões de formação estelar. Estes locais que circundam os starbursts são os principais responsáveis pela opacidade da galáxia (Guimarães, 2006).

bandas de PAHs têm sido muito usadas para se estudar a evolução de galáxias. Geralmente, galáxias com emissão no IR, tais URLIGs e starbursts, possuem a banda de $7.7 \mu \mathrm{m}$ como a mais intensa, sendo muito empregada nos diagnósticos. Embora estas bandas não sejam sempre indicadoras diretas da taxa de formação estelar (afinal, a radiação UV absorvida pelos PAHs pode ser de AGNs, de estrelas jovens e/ou de estrelas velhas em 


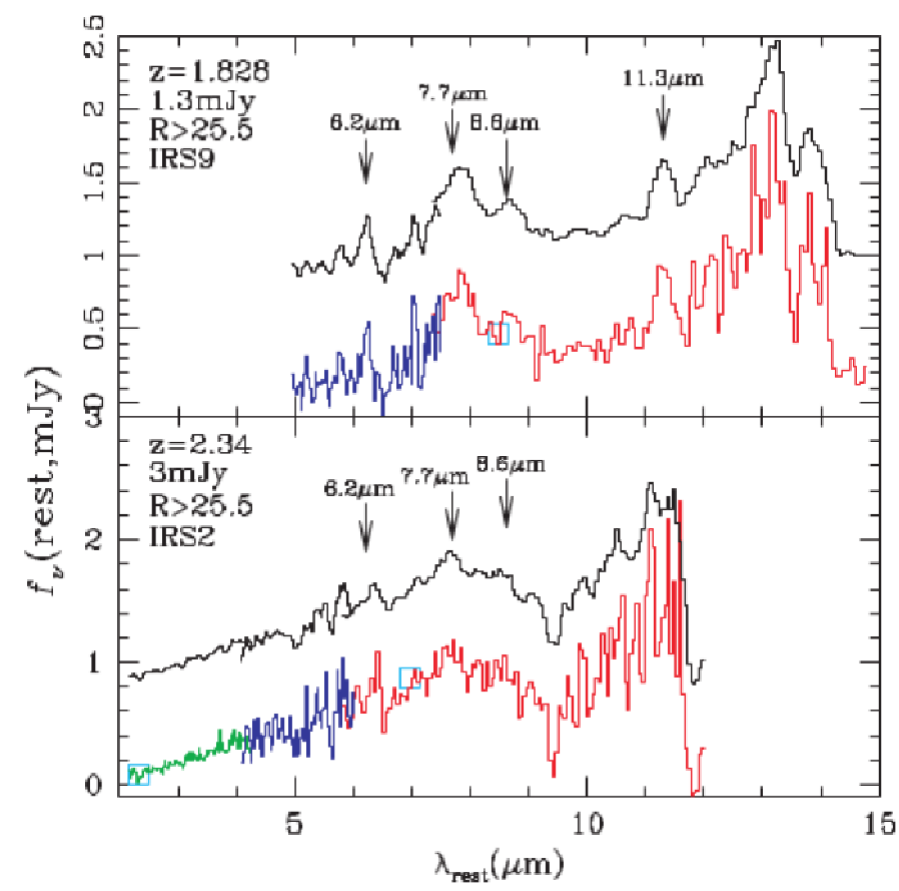

Figura 1.4: Dois espectros são apresentados em densidade de fluxo versus comprimento de onda, no repouso. As linhas vermelha, azul e a verde são os espectros observados na resolução original, ao passo que as linhas pretas são a versão suavisada, escaladas com uma constante arbitrária no eixo-y para uma visão mais clara. O segmento vermelho representa o espectro da primeira ordem Long-Low do IRS, o azul é da segunda ordem, e o verde é da primeira ordem do Short-Low. Os quadrados azuis são as densidades de fluxo na banda larga e no repouso computadas a partir da banda larga dos fluxos de $8 \mu m$ do IRAC, e de $24 \mu m$ do MIPS. Os comprimentos de onda estão corrigidos para o sistema de repouso (Gráfico retirado de Yan et al. (2005)). O IRS e seus módulos são apresentados na Seção 1.4 .2

estágios avançados de evolução), a razão linha/contínuo de $7.7 \mu m$ consegue distinguir se as URLIGS são alimentadas por AGNs ou starbursts. Aliás, a razão de fluxos das bandas de 6.2 e $7.7 \mu m$ também é muito relevante para avaliar a natureza da fonte de radiação UV. Outra banda fundamental é a $11.3 \mu \mathrm{m}$, observada a altos redshifts independentemente do grau de ionização dos PAHs. Por ser produzida a temperaturas (ou seus transientes) menores que as da banda $7.7 \mu m$, ela pode ser encontrada numa variedade maior de ambientes astrofísicos (Guimarães, 2006).

\subsection{O Mundo Aromático e os PANHs}

Pela sua robustez, os PAHs são o material orgânico dominante no espaço (Ehrenfreund et al., 2006) e, junto com outras macromoléculas aromáticas, estão entre as mais abundantes espécies moleculares que devem ter sido transportadas aos planetas por quedas de 
meteoritos e deposição de poeira interplanetária (Ehrenfreund et al., 2002). Produzidos em outras partes do Sistema Solar ou da Galáxia, foram entregues posteriormente quase intactos em planetas como Marte e a Terra.

Os PAHs podem ter seus átomos de hidrogênio ou carbono substituídos por nitrogênio, gerando PANHs (sigla em inglês para heterociclos policíclicos aromáticos nitrogenados). Uma parte considerável destes PANHs deve ter sido formada sobre os gelos depositados sobre os grãos do meio interestelar. Eles podem constituir uma etapa de canais de produção de moléculas heterocíclicas nitrogenadas. Algumas destas últimas são de grande interesse astrobiológico, por poderem desempenhar um papel pré-biotico nas origens da vida antecedendo o mundo RNA/DNA (Ehrenfreund et al., 2006), tanto na Terra como em outros ambientes astrofísicos.

Este estágio precedente ao mundo RNA/DNA foi proposto por Ehrenfreund et al. (2006) como o "Mundo Aromático", no qual uma protocélula de PAH poderia conseguir digerir e transformar recursos em blocos construtores para o crescimento e divisão da mesma. Esta também possuiria um replicador genético, imperfeito, que poderia catalizar parte destes processos. Ainda de acordo com Ehrenfreund et al. (2006), PAH carregados e hidrocarbonetos aromáticos representariam os possíveis papéis numa protocélula de recipientes, polímeros informacionais e componentes do sistema metabólico (como transdutores e interceptores de energia).

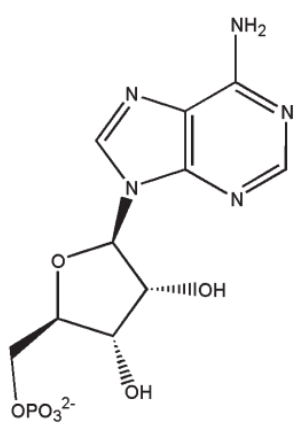

RNA

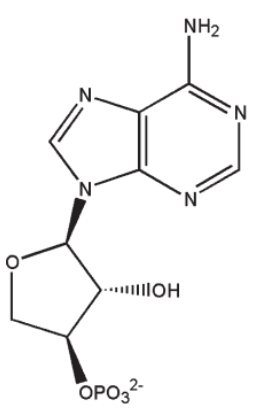

TNA

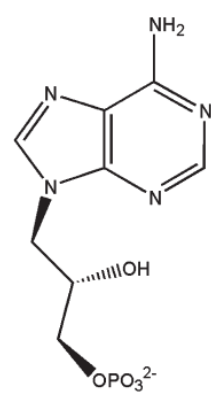

GNA

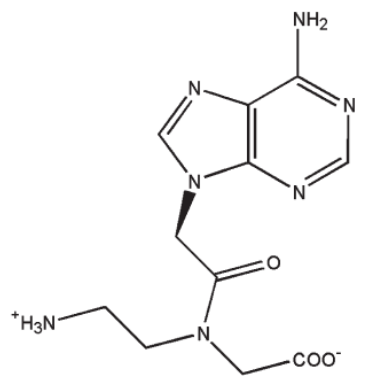

PNA

Figura 1.5: Possíveis monômeros que poderiam ter construído uma molécula genética primordial (Figura extraída de Ehrenfreund et al. (2006)). Vale salientar a presença do nitrogênio incorporado ao anel aromático em todos eles. RNA - ácido ribonucleico; TNA - ácido nucleico de treose; GNA - ácido glicol nucleico; e PNA - ácido nucleico peptídico.

Logo, estas substâncias podem fornecer o elo perdido entre a química dos abundan- 
tes PAHs no meio interestelar e as nucleobases que constituem o maquinário codificador da vida terrestre. Nucleobases nada mais são do que estruturas aromáticas compostas por moléculas monocíclicas ou bicíclicas - pirimidina e purina, respectivamente - que compõem os blocos básicos dos nucleotídios do RNA (Parker et al., 2015 a). Elas e outros heterocíclicos nitrogenados (Figura 1.5), devido à sua importância pré-biótica, devem ser investigadas com atenção, tanto considerando vias para a sua produção, como a sua estabilidade perante os campos de radiação astrofísicos (Peeters et al., 2005).

Da mesma maneira que os PAHs, PANHs podem ser sintetizados por abstração de hidrogênio e adição de acetileno (processo HACA com o radical fenil), em temperaturas de alguns milhares de Kelvin (Parker et al., 2015 a). Além disso, a substituição intermediária do acetileno pelo $H C N$ pode incorporar o nitrogênio diretamente no $\mathrm{PAH}$ ainda em crescimento (Ricca et al., 2001). Este processo, envolvendo radicais de piridil $\left(\mathrm{C}_{5} \mathrm{H}_{4} \mathrm{~N}\right)$ e o acetileno, gerando (iso)quinolina $\left(C_{9} H_{7} N\right)$, também foi mostrado para temperaturas de até $1500 K$ (Parker et al. 2015 b). Outra opção seria várias reações em fase gasosa mediadas por radicais que incorporam o nitrogênio em uma molécula aromática bicíclica (Parker et al., 2015 a).

Para temperaturas baixas, como $10 K$ de nuvens moleculares frias e até menores, reações de radicais de piridil com o 1,3 - butadieno por um evento de colisão única podem criar PANHs. Estes podem ser condensados em mantos de gelo de grãos de poeira interestelares onde podem formar estruturas de nucleobases por reações de substituição por meio de química de não-equilíbrio (Parker et al., 2015 b). Recentemente, outro estudo sugeriu a formação destas moléculas via irradiação UV de benzeno e naftaleno em misturas de gelo contendo amônia e água (Materese et al., 2015). Por outro lado, Ota (2016) propõe a criação de PANHs por choques de nitrogênio e fótons altamente energéticos.

\subsection{Observando PAHs e PANHs}

\subsubsection{As Classes de Peeters}

Variações espectrais observacionais podem ser divididas, basicamente, em duas categorias (Tielens, 2008) - variações na força relativa de diferentes características de emissão IR e variações na posição do pico e no perfil das características. Neste trabalho, o foco está na segunda. 
Diferentemente dos perfis dos modos de vibração $\mathrm{CH}$, que variam muito pouco, os dos modos CC da região de 5 a $9 \mu m$ são altamente mutáveis, mesmo para dados de baixa resolução. A tabela abaixo (Tabela 1.1) exemplifica algumas bandas e os modos de vibração à que estão ligadas. É interessante notar que as variações nas posições dos picos do modo CC estão conectadas, de forma que as bandas de $6.2 \mu \mathrm{m}$ e $7.7 \mu \mathrm{m}$ se deslocam de maneira semelhante entre os objetos. Contudo, os modos $\mathrm{CH}$ e CC não estão necessariamente interligados (Van Diedenhoven et al. 2004).

Tabela 1.1 - Modos de vibração de algumas bandas de PAHs (Hudgins e Allamandola. 1999. Tielens, 2005).

\begin{tabular}{lc}
\hline \hline Intervalos $(\mu m)$ & Modo de vibração \\
\hline $3.2-3.4$ & CH stretching \\
$6.1-6.5$ & CC stretching \\
$6.5-8.5$ & CC stretching + CC in-plane bending \\
$8.3-8.9$ & CC in-plane wagging \\
$11.0-15.0$ & CH out-of-plane bending \\
& \\
\hline
\end{tabular}

Quanto às diferenças entre os perfis, elas podem ser oriundas das condições físicas locais, do efeito acumulado de processamento nas regiões onde a emissão se originou e/ou da composição da família, tamanho molecular, carga, geometria e heterogeneidade do PAH (Sales et al., 2012). De acordo com Hudgins e Allamandola (1999), PAHs com cerca de 20 átomos de carbono já contribuiem para a emissão em $6.2 \mu m$ e, além disso, o espaçamento entre as bandas aumenta com o tamanho molecular. As bandas de 6.2, 7.7 e 11.2 $\mu \mathrm{m}$ foram classificadas por Peeters et al. (2002) em três grupos (Figura 1.6):

\section{- Classe A}

- banda $6.2 \mu m$ : pico varia até $6.23 \mu m$ e perfil apresenta uma ascensão azul aguda e uma cauda vermelha;

- Banda de 7.7 $\mu m$ : subcomponentes em 7.6 e $7.8 \mu m$;

- Derivada de material interestelar iluminado por estrelas - regiões HII, nebulosa de reflexão e meio interestelar em geral (da Galáxia e de outras); 


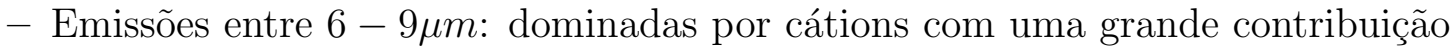
de PANHs para a banda $6.2 \mu m$;

- Emissões entre $11-15 \mu m$ : grandes PAHs neutros dominam;

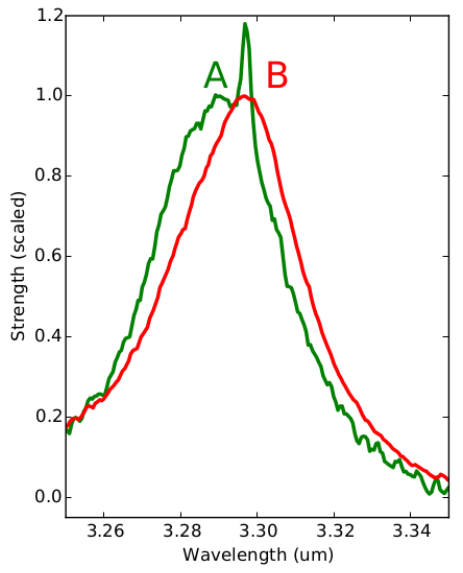

(a)

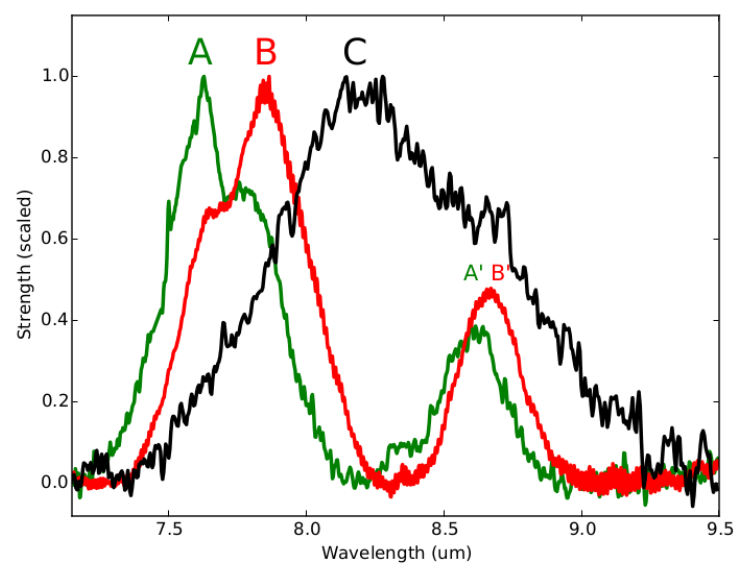

(c)

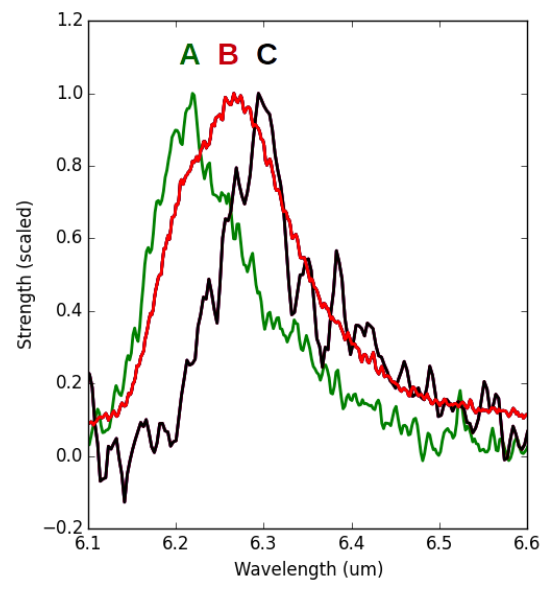

(b)

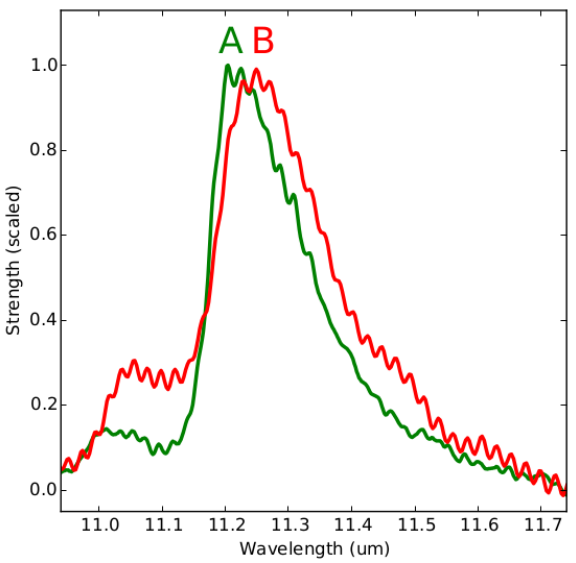

(d)

Figura 1.6: Visão geral das possíveis variações no perfil das bandas (a) $3.3 \mu m$, (b) $6.2 \mu \mathrm{m}$, (c) $7.7 \mu \mathrm{m}$ e (d) $11.2 \mu \mathrm{m}$, divididas em três classes (A, B e C) ilustradas pelos espectros normalizados dos objetos: A - região CHII IRAS 23133+6050 ((b) e (c)) e Orion Bar H2S1 ((a) e (d)); B - estrela Pós-AGB HD 44179; e C - estrela Pós-AGB IRAS 13416 - 6243 (Peeters et al., 2002; Van Diedenhoven et al., 2004).

\section{- Classe B}

- banda $6.2 \mu m$ : pico varia de 6.23 até $6.29 \mu \mathrm{m}$ e perfil é mais simétrico do que o da classe A;

- Banda de $7.7 \mu m$ : subcomponentes em 7.6 e $7.8 \mu m$ com grande diferença de força relativa comparada à classe $\mathrm{A}$; 
- Derivada de material circumestelar, nebulosas planetárias, diversos objetos pósAGB e estrelas Herbig AeBe;

- Emissões entre $6-9 \mu m$ : PAHs pequenos e grandes contribuem igualmente;

- Emissões entre $11-15 \mu m$ : grandes PAHs neutros dominam;

\section{- Classe C}

- banda $6.2 \mu m$ : pico cai em posições maiores que $6.29 \mu m$ e perfil é mais simétrico do que o da classe A;

- Banda de 7.7 $\mu m$ : aparentemente está deslocada para as proximidades de $8.2 \mu m$;

- Com poucas fontes, é bem difícil assegurar se ela realmente compõe outra categoria ou ainda está vinculada às anteriores;

- Derivada de uns poucos objetos pós-AGB extremamente enriquecidos por carbono;

- Emissões entre $6-9 \mu m$ : PAHs pequenos e grandes contribuem igualmente;

- Emissões entre $11-15 \mu m$ : pequenos PAHs neutros dominam;

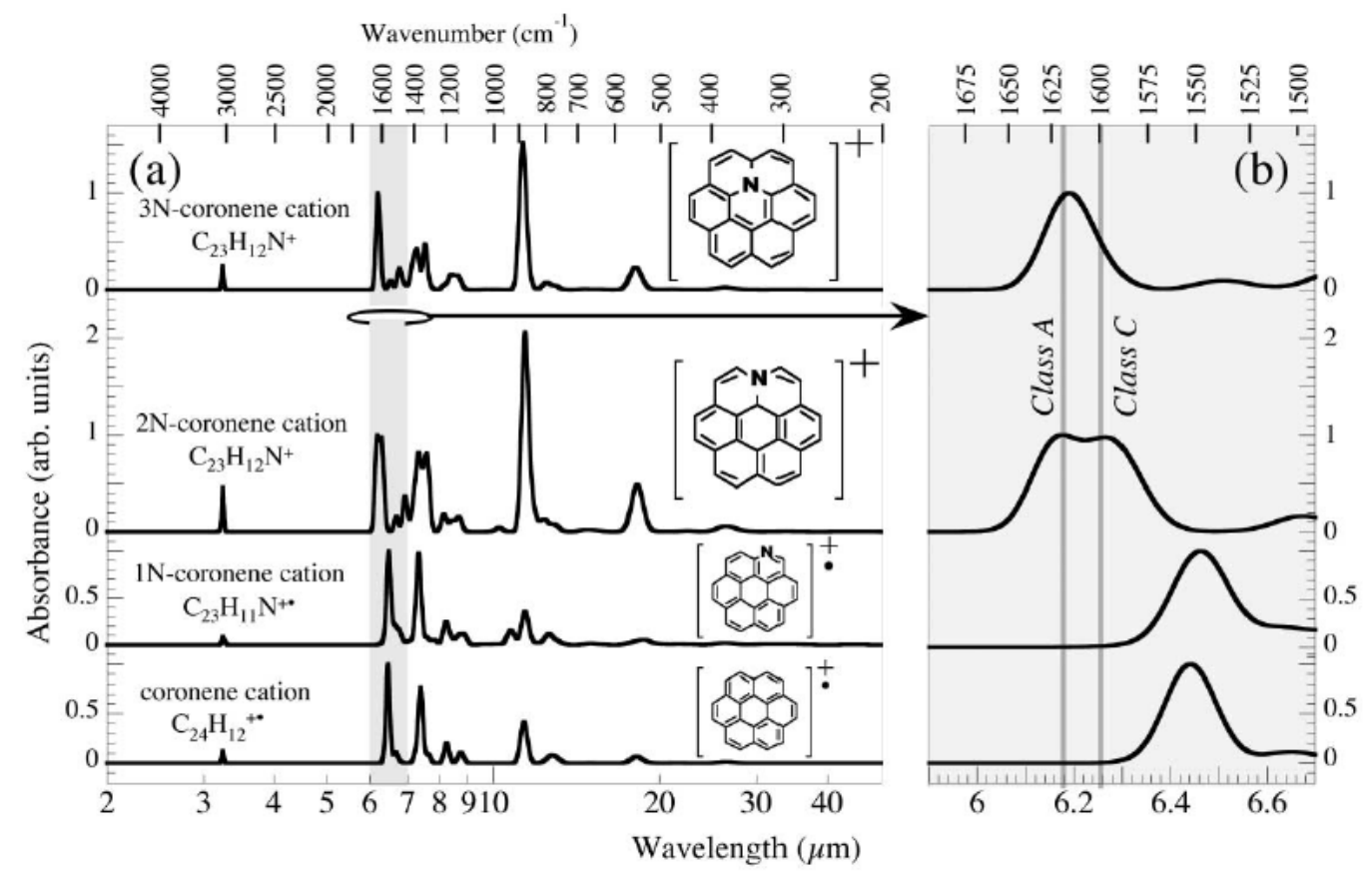

Figura 1.7: Espectros IR calculados de três possíveis cátions de coroneno com substituição de N comparados com o sem substituição. Em (a) há os espectros completos e em (b) há somente a região de $6.2 \mu m$ e as posições médias das classes A e C. Os dois últimos cátions estão denotados com um ponto preto pois possuem estruturas eletrônicas radicais (Figura retirada de Hudgins et al. (2005)). 
De acordo com Hudgins et al. (2005), o desvio da banda de 6.2 $\mu m$ para o azul (correspondente à classe A) não consegue ser esclarecido somente pelo aumento no tamanho do PAH. Nem mesmo a substituição de átomos de $S i$, a substituição periférica de átomos de $O$, a complexação com átomos de metais e a diminuição da simetria molecular conseguem reproduzir, precisamente, a posição desta banda interestelar. Aparentemente, o nitrogênio incorporado ao anel aromático (mais especificamente, quando o carbono substituído é interno - PANHs endoesqueléticos (Figura 1.7) ) é o único capaz de reproduzir as observações e satizfazer as restrições astrofísicas envolvidas nesta questão simultaneamente. Ainda segundo Hudgins et al. (2005), estes resultados permitem adquirir o grau de substituição de $N$ na população de PAH e inferir um limite mínimo de 1 a $2 \%$ do $N$ cósmico capturado em PANHs.

Assim, a análise dos perfis e a interpretação da variação do perfil da AIB a $6.2 \mu m$, em relação ao esperado de um PAH simples, torna possível determinar a presença de nitrogênio incorporado ao PAH. Isto abre uma nova janela para o censo do nitrogênio no universo, pois representaria um reservatório adicional deste elemento, em condições de densidade e temperaturas distintas daquelas da fase gasosa e em gelos.

\subsubsection{Spitzer Space Telescope e o IRS}

Como as principais bandas de PAH no IR estão entre 3 a $20 \mu m$, o Telescópio Espacial Spitzer é um dos telescópios que permitem a obtenção de dados a respeito destas moléculas. Lançado em 25 de Agosto de 2003, é uma missão da NASA gerenciada pelo Jet Propulsion Laboratory e foi o maior telescópio infravermelho já enviado ao espaço até esta data. Com $85 \mathrm{~cm}$ de espelho, possui três instrumentos científicos - IRAC (Infrared Array Camera), IRS (Infrared Spectrograph) e MIPS (Multiband Imaging Photometer for Spitzer) - que adquiriram imagens e espectros de 3 a $180 \mu m$ com uma resolução de 2 arcosegundos para comprimentos de onda menores a 40 arcosegundos para os maiores 1 . Suas capacidades variam de 3 a $180 \mu m$ para imageamento e fotometria, de 5 a $40 \mu m$ para espectroscopia e de 50 a $100 \mu m$ para espectrofotometria $2^{2}$. Atualmente, somente o IRAC está em funcionamento.

A Figura 1.8 mostra os compartimentos da espaçonave que abriga o telescópio e seus

\footnotetext{
${ }^{1}$ http://irsa.ipac.caltech.edu/onlinehelp/heritage/\#id=about, acessado em Fevereiro, 2016.

${ }^{2}$ http://www.spitzer.caltech.edu/info/277-Fast-Facts, acessado em Fevereiro, 2016.
} 


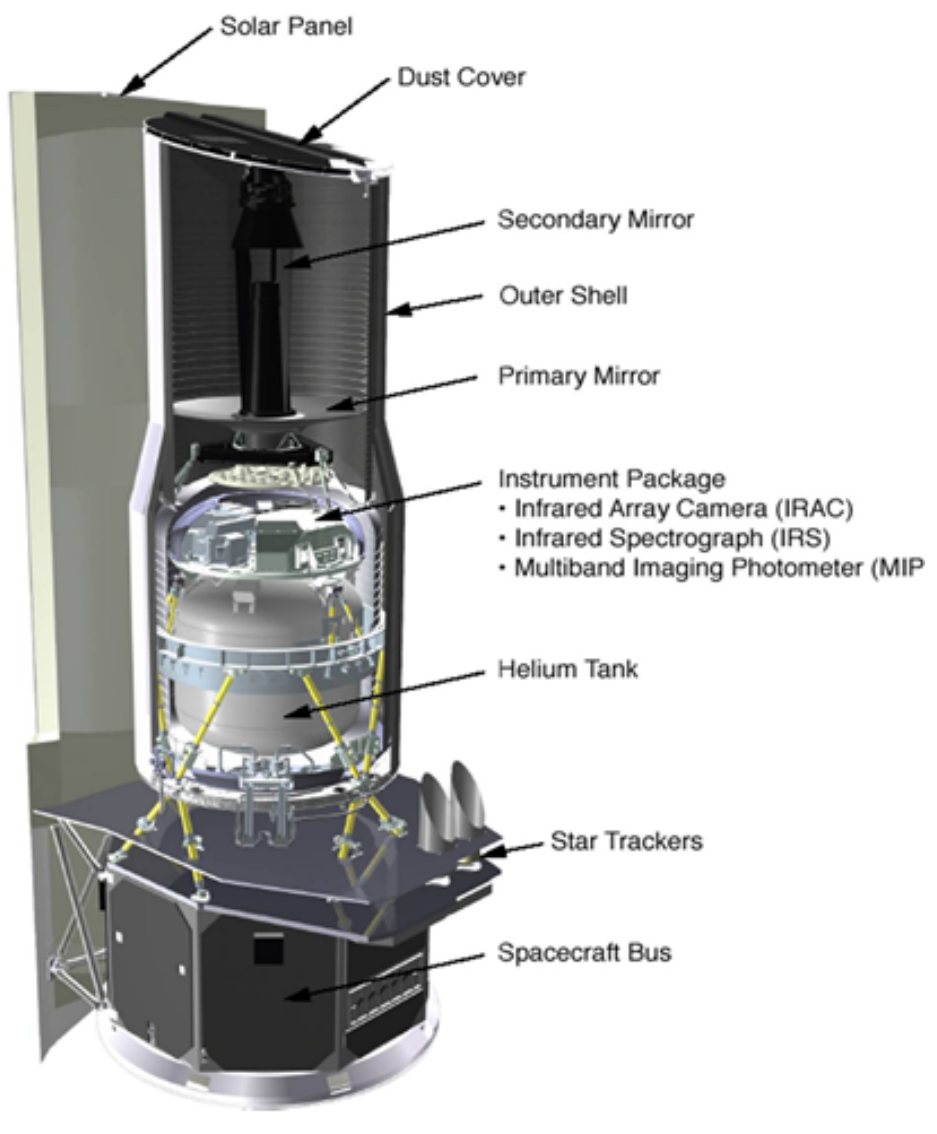

Figura 1.8: Distribuição interna do observatório Spitzer, com cerca de $4.5 m$ de altura e $2.1 m$ de diâmetro. Quando lançado, sua massa era de $861 \mathrm{~kg}$. Imagem extraída do Spitzer Telescope Handbook.

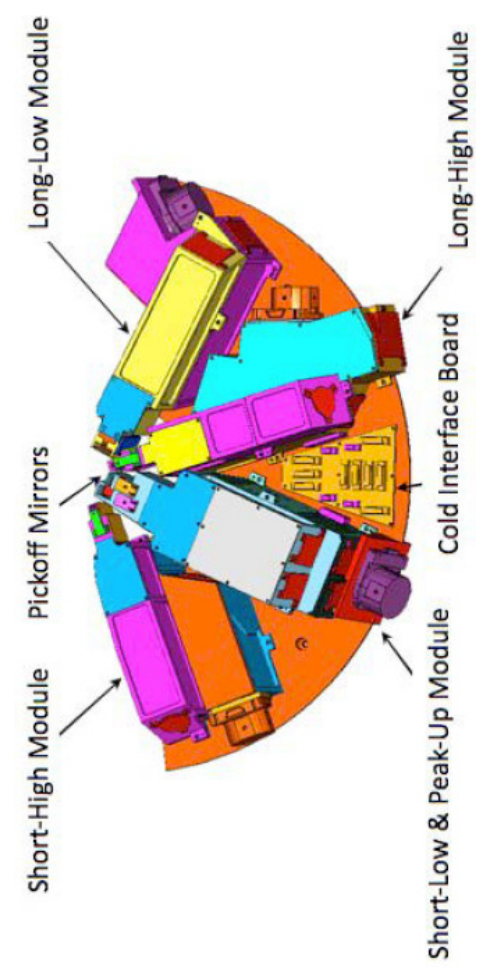

Figura 1.9: Distribuição dos módulos de observação do IRS. Imagem extraída do IRS Instrument Handbook.

instrumentos. Por outro lado, a Figura 1.9 se refere à distribuição dos equipamentos do IRS, cujas observações foram utilizadas neste trabalho e, portanto, será dada uma ênfase maior a ele.

Todos os dados coletados estão disponibilizados gratuitamente no Spitzer Heritage Archive (SHA) do NASA/IPAC Infrared Science Archives (IRSA) ${ }^{3}$ tanto as observações brutas como imagens já previamente revisadas pelo Spitzer Science Center (SSC). Mais informações sobre a missão, os instrumentos e o acesso aos dados podem ser obtidas através da página da web do $\mathrm{SSC}_{4}^{4}$

O IRS5 contém três modos de observação - fixo (Staring Mode), de mapeamento (Mapping Mode) e o Peak-up Imaging. O último consiste em dois filtros em 16 e $22 \mu m$ que

\footnotetext{
${ }^{3}$ http://sha.ipac.caltech.edu

${ }^{4}$ http://ssc.spitzer.caltech.edu/

${ }^{5}$ http://irsa.ipac.caltech.edu/data/SPITZER/docs/irs/
} 


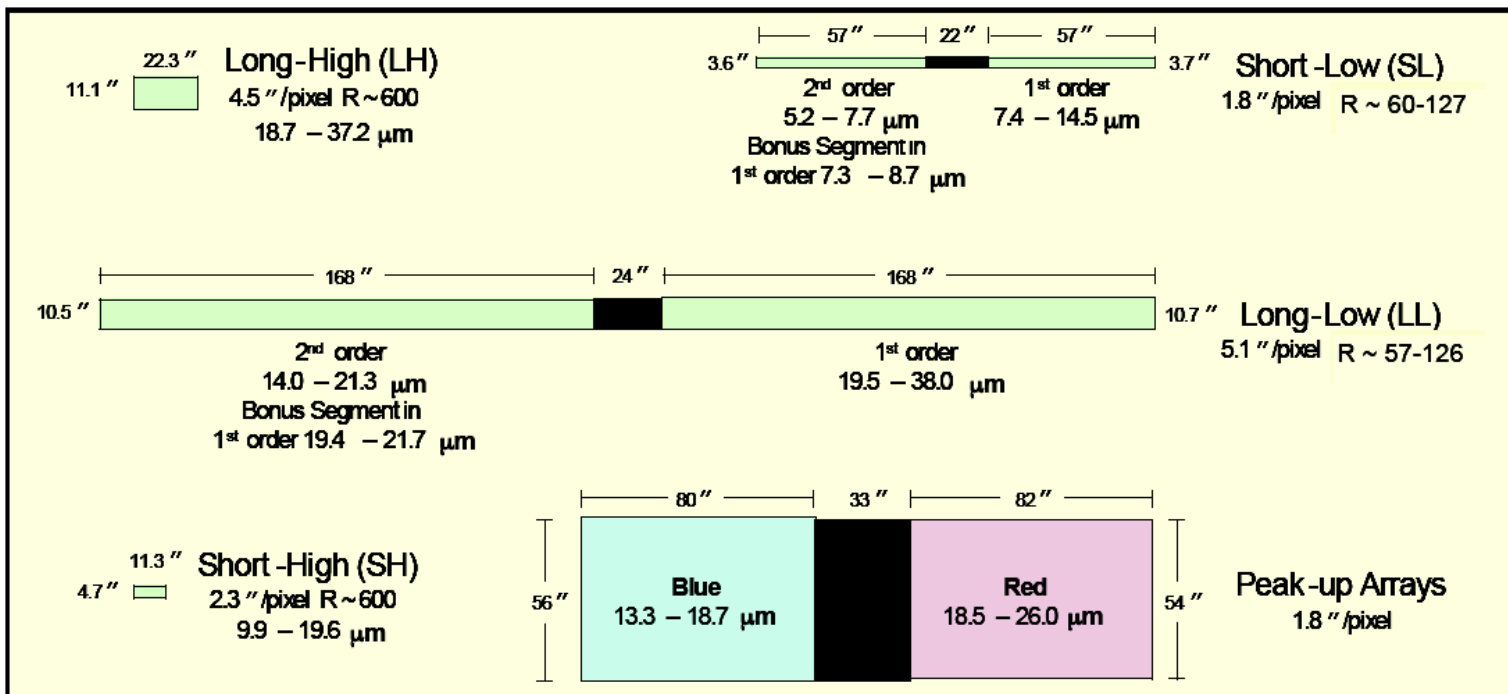

Figure 1: Summary of IRS module properties. The slits are not parallel as depicted in this figure. Actual slit position angles relative to a Spitzer roll angle of $0^{\circ}$ are $S L=+84.7^{\circ}, L L=+181.2^{\circ}$, $\mathrm{SH}=+221.5^{\circ}, \mathrm{LH}=+136.7^{\circ}$, and IRS Peak-up $=+177.0^{\circ}$.

Figura 1.10: Resumo das propriedades dos módulos do IRS. Vale ressaltar que os módulos não são paralelos, eles estão distribuídos conforme a Figura 1.9. Imagem extraída do IRS Pocket Guide v3.1 (Agosto, 2007).

servem ou para identificar fontes pontuais e colocá-las no meio da fenda do IRS, ou para fazer fotometria. Enquanto isso, os dois primeiros são usados para espectroscopia com quatro módulos de resolução, explicados com maiores detalhes na Figura 1.9. Eles estão distribuídos em:

Short-Low (SL) Comprimentos de onda baixos e resolução baixa;

Long-Low (LL) Comprimentos de onda grandes e resolução baixa;

Short-High (SH) Comprimentos de onda baixos e resolução alta;

Long-High (LH) Comprimentos de onda grandes e resolução alta.

A baixa resolução está entre $R \sim 20-130$ e a 'alta' por volta de $R \sim 600$, cobrindo uma região de 5.2 a $38 \mu m$. Os módulos de baixa resolução têm um esquema de fenda longa que possibilita a obtenção de informações espectrais e espaciais unidimensionais ao mesmo tempo. Além disso, a fenda está dividida em duas sub-fendas alinhadas, que proporcionam espectros de primeira e segunda ordem. Por sua vez, os módulos de alta resolução são qualificados para fornecer ampla cobertura espectral numa única exposição 6 .

\footnotetext{
${ }^{6}$ http://irsa.ipac.caltech.edu/data/SPITZER/docs/irs/irsinstrumenthandbook/
} 


\subsection{Visão geral dos objetivos}

Este trabalho pretente verificar a viabilidade do Mundo Aromático proposto por Ehrenfreund et al. (2006) buscando-se os PANHs, que podem estar conectados com moléculas pré-bióticas. Através da interpretação do perfil da banda de $6.2 \mu m$, que pode indicar a presença dos PANHs, também será possível dividir as fontes estudadas de acordo com as classes de Peeters. Isto possibilitará um entendimento a cerca da distribuição de PAHs, fornecendo perspectivas da evolução química e, porventura, das origens da vida (como conhecemos) nestes ambientes.

\subsection{Organização da Dissertação}

Esta dissertação está distribuída da seguinte forma: no Capítulo 2 são discutidos os objetos selecionados para este trabalho e como foram escolhidos; no Capítulo 3 são apresentadas as diferentes análises conduzidas sobre os alvos e quais resultados foram encontrados para cada uma; e no Capítulo 4 são expostas as conclusões e as perspectivas do trabalho. Quanto aos apêndices, neles são mostrados uma lista com as abreviações utilizadas (Ap. A), gráficos obtidos durante as análises (Ap. B e C e tabelas referentes às fontes (Ap. D). 
Capítulo 2

\section{Os Alvos}

\subsection{Primeiras opções}

Com o intuito de se analisar a característica do perfil de $6.2 \mu m$, procurando possíveis discrepâncias que estejam relacionadas com um átomo de nitrogênio presente numa molécula de PAH, os mesmos alvos de Yan et al. (2007) poderiam proporcionar um grande intervalo de redshift e uma futura comparação de resultados, enriquecendo o trabalho. Das 52 fontes do artigo, 13 apresentam fortes bandas de PAHs, são muito brilhantes e classificadas como ULIRGs. Por isso, foram as selecionadas inicialmente. Seus nomes e AORKEYs (número exclusivo de identificação da observação dentro da missão Sptizer) se encontram na Tabela 2.1 .

Tabela 2.1 - Nomes e redshifts das ULIRGs utilizadas por Yan et al. (2007).

\begin{tabular}{lcc}
\hline \hline & & \\
MIPS ID & AORKEY & $z_{v i s} \pm 1 \rho$ \\
\hline MIPS 15928 & 11856384 & $1.52 \pm 0.02$ \\
MIPS 22651 & 11859456 & $1.73 \pm 0.02$ \\
MIPS 22277 & 11866112 & $1.77 \pm 0.03$ \\
MIPS 289 & 11864320 & $1.86 \pm 0.01$ \\
MIPS 22530 & 11865856 & $1.96 \pm 0.01$ \\
MIPS 16144 & 11862016 & $2.13 \pm 0.02$ \\
MIPS 506 & 11858432 & $2.52 \pm 0.02$ \\
MIPS 16059 & 11864832 & $2.43 \pm 0.07$ \\
MIPS 180 & 11860736 & $2.47 \pm 0.04$ \\
MIPS 8327 & 11865344 & $2.48 \pm 0.06$ \\
MIPS 8242 & 11868672 & $2.45 \pm 0.04$ \\
MIPS 22699 & 11857920 & $2.59 \pm 0.04$ \\
MIPS 8342 & 11864064 & $1.57 \pm 0.09$ \\
& & \\
\hline
\end{tabular}


Os dados foram baixados do SHA $]^{1}$ já com a primeira redução do SSC pipeline - BCD (2D Basic Calibrated Data). Nela estão inclusas correções como de flatfield, calibração de fluxo e subtração de dark sky. Maiores detalhes destas reduções estão presentes no IRS Instrument Handbook2.

Infelizmente, apenas o pipeline do SSC não é o suficiente para as fontes porque elas são muito fracas. Uma nova redução de dados é necessária para extrair o background remanescente, mascarar os rogue pixels e sobrepor as imagens para aumentar o sinal-ruído.

\subsubsection{Redução de dados}

\subsubsection{Background}

O background é construído a partir das imagens BCDs disponibilizadas pelo IRSA (são as imagens com a terminação *bcd.fits). Há, basicamente, duas formas de se fazer isto:

1. mascarar o espectro do alvo na imagem BCD, combinar as imagens de ambas posições de nod por mediana e subtrair a imagem resultante de todas as *bcd.fits. Ainda pode ser necessário verificar se existem espectros de outros objetos, que devem ser mascarados antes da extração (Yan et al., 2007);

2. utilizar a parte da fenda que esteja em nod-off (onde o alvo não se encontra) das imagens, combinando-as por mediana. Em seguida, subtrair das imagens. Isto deve ser feito para as duas posições de nod. Contudo, não é aconselhada para fontes fracas em que possam haver outros espectros poluindo o sinal (Wu et al., 2010).

Em algumas imagens, não é possível se afirmar onde exatamente está o espectro (por exemplo, Figura 2.1. Sem isso, não se pode mascará-lo. Outra questão é qual programa usar para fazer a redução. Nenhum dos artigos mencionam se foi utilizado o IRAF (Image Reduction and Analysis Facility), o IDL (Interactive Data Language) ou mesmo outro. Os manuais, tanto o IRS Instrument Handbook como o Spitzer Data Analysis Cookbook, não fornecem informações mais aprofundadas quanto a utilização dos programas que não pertencem ao SSC. A Figura 2.2 mostra um teste realizado escolhendo-se a primeira alternativa.

\footnotetext{
${ }^{1}$ http://sha.ipac.caltech.edu/applications/Spitzer/SHA/, acessado em Março, 2014

2 http://irsa.ipac.caltech.edu/data/SPITZER/docs/irs/irsinstrumenthandbook/
} 


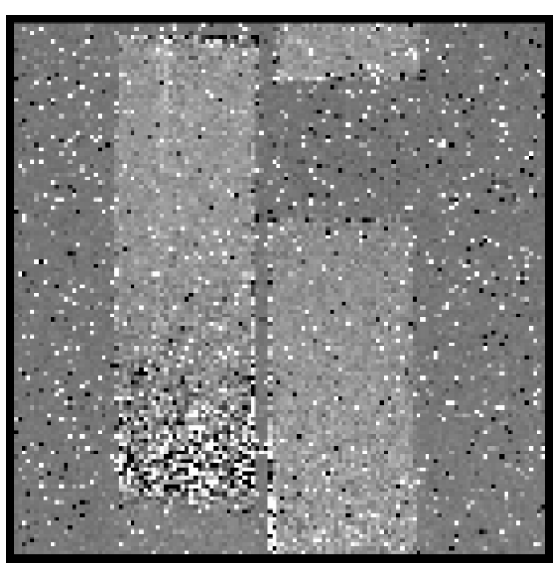

Figura 2.1: Imagem BCD do objeto MIPS 289. Como se pode perceber, é muito difícil verificar, visualmente, onde está o espectro na imagem com exatidão.

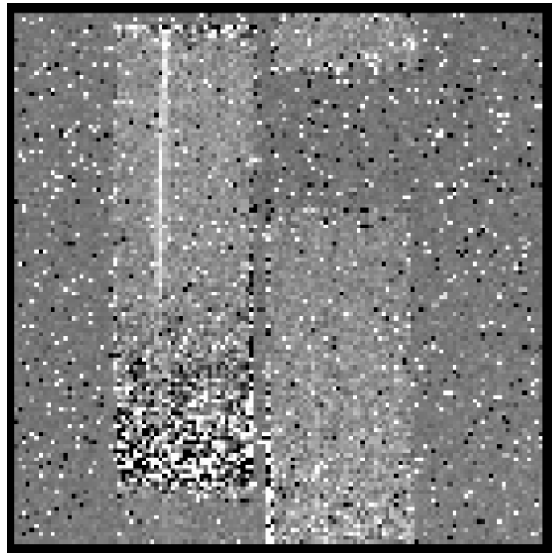

(a) Uma das imagens BCD do objeto, o espectro está na fenda esquerda.

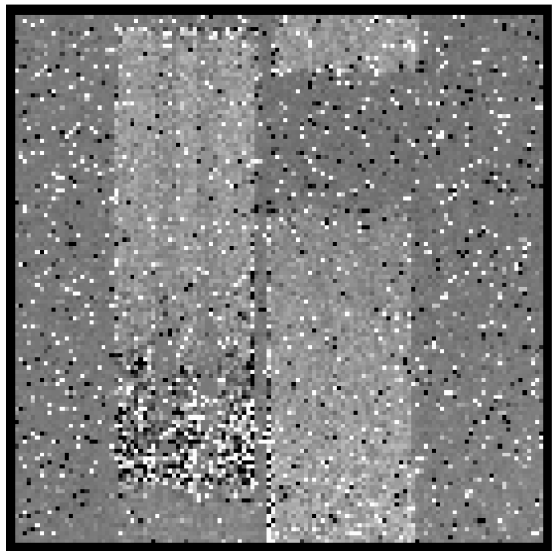

(c) Imagem do background criada por mediana a partir das imagens mascaradas do objeto.

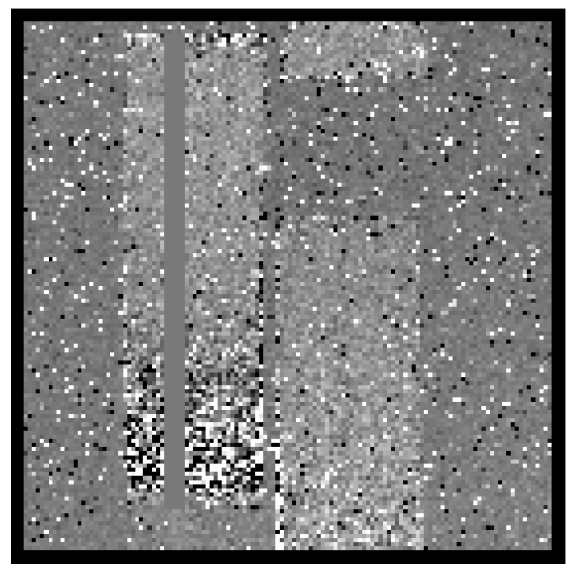

(b) Mesma imagem, mas com o espectro mascarado manualmente.

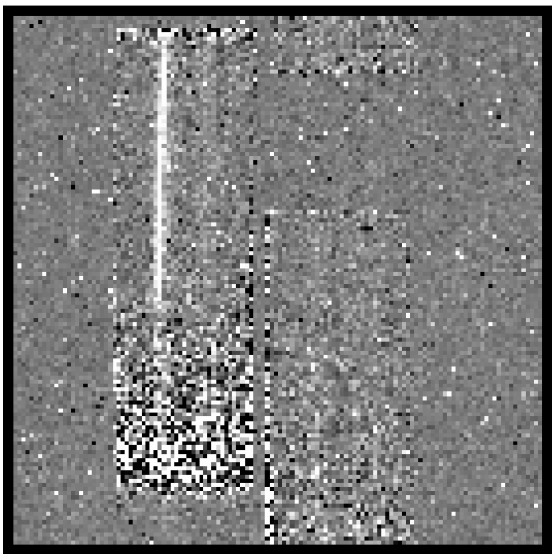

(d) Mesma imagem de (a) e (b), agora com o background subtraído.

Figura 2.2: Teste de redução do background com o objeto MIPS 78 de Yan et al. (2005) segundo a primeira opção descrita. O programa usado para mascarar foi o IDL e o restante foi realizado com o IRAF. 


\subsubsection{Rogue pixels}

Rogue pixels são aqueles cuja corrente de escuro é anormalmente alta e varia com o tempo e com backgrounds de céus diferentes, destoando demais do background local. Eles podem ser identificados manualmente, computando a dispersão de ruído ao redor de cada pixel e sinalizando os que possuem valor acima de $5 \rho$ do valor médio (Yan et al., 2007).

Outra maneira, aderida por Wu et al. (2010), é usar a ferramenta IRSCLEAN disponibilizada pelo SSC. Baseada em IDL, ela permite a criação e edição de máscaras de bad pixel e rogue pixel, em ambos os modos automático e manual. Aliás, poderia também mascarar os espectros para a construção do background.

\subsubsection{Coad}

Para sobrepor as imagens tratadas, o ideal é usar outra ferramenta do SSC - o coad. Também feito em IDL, ele sobrepõe as imagens BDCs do Spitzer juntamente com suas respectivas máscaras ( ${ }^{*}$ fmask.fits) e incertezas (*func.fits). As duas vêm no pacote de dados com as BCDs, e devem ser consideradas no tratamento. Segundo o IRS Instrument Handbook, as incertezas são propagadas em quadratura. Com o Coad, este processo é automático e o header das imagens não altera, o que é fundamental para o SPICE. É importante ressaltar que não se sobrepoem imagens de diferentes posições de nod. O espectro é extraído de cada nod e, apenas depois, eles são combinados para formar um espectro único.

\subsubsection{Extração espectral}

Para a extração, pode se utilizar o software SPICE (Spitzer IRS Custom Extraction) e as imagens finais $2 \mathrm{D}$ com suas respectivas máscaras e incertezas, obtidas através dos passos descritos anteriormente. O SPICE é baseado na linguagem JAVA e foi criado pelo SSC para reduzir dados obtidos pelo IRS. A Figura 2.3 mostra seu logotipd ${ }^{3}$. Os objetos podem ser tratados como fontes pontuais e se pode usar o modelo point source with optimal extract. Segundo Yan et al. (2007), uma abertura de extração de 6 pixels em $27 \mu \mathrm{m}$ minimiza as incertezas do background e maximiza o sinal-ruído para espectros fracos, para estes dados.

Outra opção seria a ferramenta SMART (Spectroscopic Modeling Analysis and Reduc-

\footnotetext{
${ }^{3}$ http://irsa.ipac.caltech.edu/data/SPITZER/docs/dataanalysistools/tools/spice/
} 
tion Tool) (Figura 2.4 de Cornell para processar observações do IRS do Spitzer (Lebouteiller et al., 2010), especialmente para fontes fracas ou extensas (Higdon et al., 2004). Este software trabalha diretamente com as imagens BCDs e abrange diversas análises de dados em tempo real, tais como operações de imagens (mediana, subtração múltipla, ...), diferentes tipos de extrações espectrais (para fontes pontuais, extensas ou mesmo múltiplas) e fotometria. Inclusive, permite a subtração do background a partir de imagens do alvo em posições diferentes, a limpeza com IRSCLEAN e a sobreposição dos espectros finai:5. Assim, todos os passos anteriores podem ser realizados diretamente com o SMART, tornando-o a melhor e mais segura escolha de redução. Um exemplo detalhado deste procedimento para uma fonte pontual, como a dos alvos selecionados, foi disponibilizado no websit $\epsilon^{6}$ do SMART.

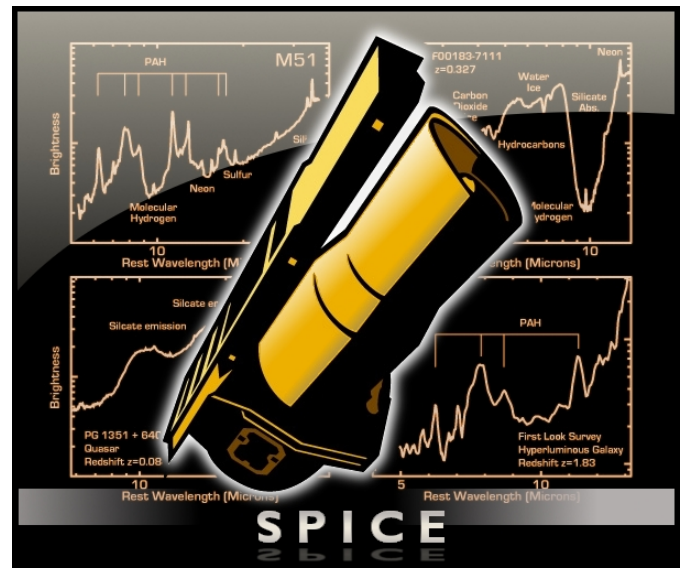

Figura 2.3: Logotipo do SPICE

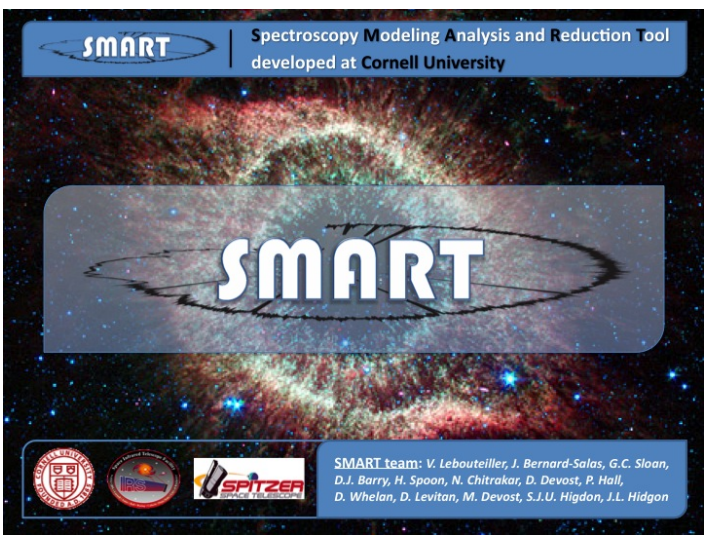

Figura 2.4: Logotipo do SMART

\subsubsection{Considerações}

A Figura 2.5 mostra uma tentativa de extração espectral do objeto MISP289. Se comparada com o espectro superior da Figura 1.4. retirada de Yan et al. 2005) que nomeou este alvo como IRS9, pode-se notar a diferença entre os dois. Por serem reduções diferentes, este fato já era esperado. Contudo, isto também pode indicar uma possível variação na posição central da banda de $6.2 \mu m$, dificultando futuras análises.

Além disso, outra questão foi levantada. Estes objetos escolhidos tiveram seus redshifts extrapolados pelas bandas de emissão dos PAHs, com respectivas incertezas variando de

\footnotetext{
${ }^{4}$ http://isc.astro.cornell.edu/SmartDoc/WebHome

${ }^{5}$ http://isc.astro.cornell.edu/SmartDoc/WebHome

${ }^{6}$ http://isc.astro.cornell.edu/SmartDoc/ScreenShotExample
} 


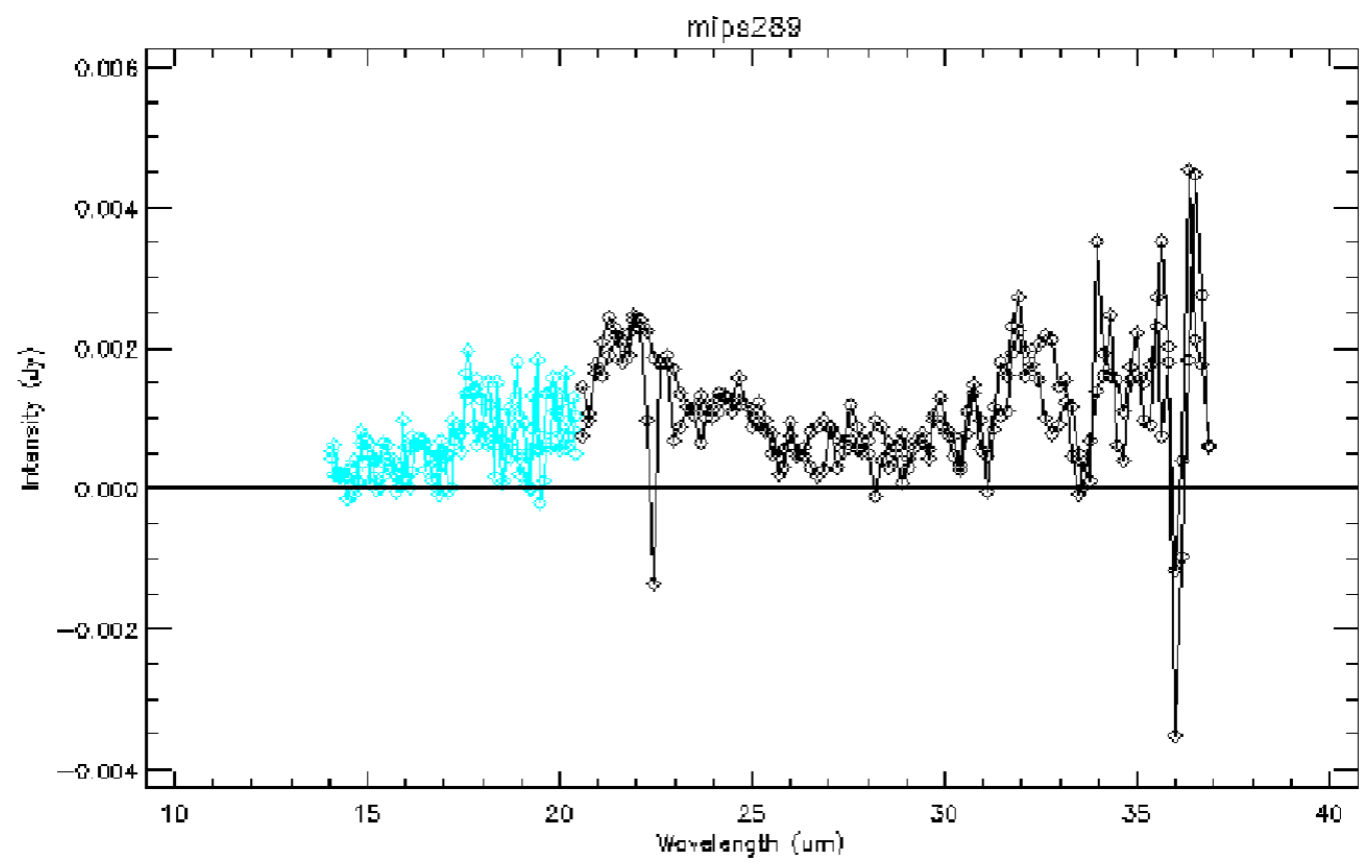

Figura 2.5: Espectro da fonte MIPS289 extraído com o SMART. A linha preta é a primeira ordem do módulo Long-Low do IRS e a azul claro é a sua segunda ordem.

0.01 à 0.2 (Yan et al., 2007). E, para se analisar o pico da banda de $6.2 \mu m$, primeiramente é necessário fazer a correção de redshift. Logo, estes dados não são precisos para esta avaliação, uma vez que o redshift é definido a partir das bandas a serem estudadas e ainda possuem altas incertezas. Até poderiam ser usados como estatística para próximos estudos, mas somente 13 fontes não resultariam numa boa estimativa. A solução foi se utilizar uma nova abordagem, discutida em seguida.

\subsection{O projeto Spitzer/IRS Atlas}

O projeto Spitzer/IRS ATLAS, criado por Hernán-Caballero e Hatziminaoglou (2011), possui 739 espectros já reduzidos de diversos tipos de objetos extragalácticos, tais como galáxias Seyfert, radiogaláxias e galáxias submilimétricas. Em especial, galáxias ativas e starbursts. Ele é uma compilação de dados fotométricos e espectroscópicos juntamente com suas respectivas propriedades espectrais e dados auxiliares (redshift, fotometria óptica, etc.) 7. Todos foram observados com o espectrógrafo IRS do telescópio Sptizer, em baixa, média e alta resolução, e extraídos dos artigos recentes 8 da literatura através das figuras postscript

\footnotetext{
${ }^{7}$ http://www.denebola.org/atlas/?p=intro, acessado em Abril/2015.

${ }^{8}$ http://www.denebola.org/atlas/?p=data\#references, acessado em Abril, 2015.
} 
enviadas para o serviço de pré-impressão do astro-ph ${ }^{9}$ pelos autores. Por esta razão, eles apresentam uma qualidade inferior quando comparados aos espectros propriamente e completamente reduzidos a partir do pipeline do SSC. Em alguns casos, as incertezas no fluxo estão ausentes ou os espectros foram suavizados. Contudo, para um trabalho estatístico, esta situação não interfere demasiadamente na análise.

\subsubsection{A amostra MIR starbursts}

Por todos estes motivos, foi decidido usar os dados presentes no ATLAS relacionados com starbursts, por possuírem maiores emissões de PAHs (Seção 1.2). Especificamente, uma amostra de galáxias cuja emissão foi considerada dominada por starbursts, de acordo com os critérios utilizados por Hernán-Caballero e Hatziminaoglou (2011) - MIR_SB sam$p l e^{10}$ (starbursts no infravermelho médio). Ela contém 257 galáxias com o comprimento de onda observado (sem correção de redshift). O ATLAS fornece também valores fotométricos e espectroscópicos dos redshifts, retirados dos artigos e checados com o NED (NASA Extragalactic Data base, base de dados extragalácticos da NASA), e já sugere quais valores são melhores. Estes últimos foram usados para fazer esta correção de redshift.

Também foram adicionadas as fontes de Yan et al. (2007) com fortes bandas de PAHs, e cujos dados estavam disponíveis no ATLAS (Tabela 2.1, com exceção dos objetos MIPS289, MIPS22530 e MIPS22651 que já estão presentes na amostra $M I R_{-} S B$ ). Com isso, primeiro foi visto se todas as galáxias continham, pelo menos, a banda de $7.7 \mu m$. Do contrário, elas não serviriam para este trabalho. Afinal, embora esta banda não esteja diretamente ligada aos PANHs, ela apresenta relação com a de $6.2 \mu m$ e pode ser útil em estudos futuros. Sendo assim, foram selecionadas 229 fontes, dispostas na Tabela D.1, Apêndice D.

\footnotetext{
${ }^{9}$ http://arxiv.org/archive/astro-ph

10 http://www.denebola.org/atlas/, acessado em Junho/2015
} 
Capítulo 3

\section{Análise de dados e resultados}

\subsection{PAHFIT}

PAHFIT é uma ferramenta em IDL criada para decompor espectros de baixa resolução do IRS (Spitzer) em características de poeira, contínuo estelar e de poeira térmica, absorção de silicato e emissões de linhas iônicas e moleculares (Smith et al., 2007). Para tanto, o espectro é considerado ser formado por uma emissão no contínuo ocasionada por poeira e luz estelar, emissões de linhas proeminentes, bandas de emissão individual e agregada de PAHs e uma atenuação causada por extinção de grãos de poeira. A lei de opacidade de poeira usada é de Kemper et al. (2004), cuja extinção infravermelha é uma lei de potência mais uma característica de silicato em $9.7 \mu m$ (Sales et al., 2012). No geral, o perfil de Drude ou a Gaussiana são usados para ajustar as características de poeira, a função de corpo negro para o contínuo e a Gaussiana para características iônicas e moleculares.

Além disso, o programa também consegue decompor as bandas de PAH, que apresentam seus comprimentos de onda centrais fixados no programa - uma vez que as posições destas bandas não variam significantemente 1 . Vale ressaltar que foram usados perfis de Drude para recuperar a força total das características das emissões de poeira, incluindo as asas de perfis alargados. O perfil de Drude é o perfil da frequência teórica para uma oscilador clássico amortecido, mostrado pela Equação 3.1, onde $\lambda_{r}$ é o comprimento de onda central, $\gamma_{r}$ é a FWHM fracional e $b_{r}$ é a intensidade central(Smith et al., 2007).

$$
I_{\nu}^{r}=\frac{b_{r} \gamma_{r}^{2}}{\left(\frac{\lambda}{\lambda_{r}}-\frac{\lambda_{r}}{\lambda}\right)^{2}+\gamma_{r}^{2}}
$$

\footnotetext{
${ }^{1}$ http://tir.astro.utoledo.edu/jdsmith/research/pahfit.php, acessado em Junho/2015
} 
Entretanto, como o objetivo deste trabalho é justamente estudar as variações nas posições dos picos de emissão de PAHs, o PAHFIT foi usado somente para ajustar o contínuo, a absorção de silicato e as emissões de linhas iônicas e moleculares. Estes componentes foram subtraídos posteriormente dos espectros originais para as próximas etapas da análise.

Foram feitos dois programas em IDL para se investigar todos os alvos. O primeiro, fit_pahfit.pro, usa o PAHFIT para ajustar o modelo para cada objeto e retorna uma tabela com os resultados - desde o melhor ajuste até cada componente utilizada pelo modelo. O segundo, ps_creater.pro, usa esta tabela para criar o gráfico dos resultados e salvá-lo numa imagem com formato .ps. Estes gráficos foram limitados no comprimento de onda (até $14 \mu m$ ) para melhor visualização desta região onde as bandas de PAHs são mais fortes. Os dados são representados por pontos pretos com as respectivas barras de erro verticais e as linhas coloridas representam as diferentes contribuições individuais para o espectro (Tabela 3.1).

Tabela 3.1 - Cores utilizadas nos gráficos com os ajustes do PAHFIT e as componentes do modelo à que se atribuem.

\begin{tabular}{ll}
\hline \hline Cor da linha & Componente do modelo \\
\hline Rosa & melhor ajuste obtido pelo PAHFIT \\
Verde & contribuição das bandas de PAHs \\
Azul & contribuição das linhas iônicas e moleculares \\
Vermenlha & contribuição total do contínuo \\
Laranja & componente individual de poeira térmica do contínuo \\
Amarela & componente individual de radiação estelar do contínuo \\
Tracejada preta & componentes de extinção ótica \\
& \\
\hline
\end{tabular}

No decorrer dos ajustes, notou-se que algumas emissões peculiares estavam afetando os resultados do PAHFIT. Para estudá-las, optou-se pelo método da raiz do valor quadrático médio, discutido a seguir.

\subsubsection{RMS}

Antes de se calcular os valores de RMS (Equação 3.2), foi preciso subtrair o melhor ajuste (total) do PAHFIT dos dados originais. Dessa maneira, os resíduos seriam avaliados. 
Para tanto, foi criado o programa em IDL substract_model.pro, que gera tabelas com os valores da subtração. Depois, para se determinar o RMS, foi construído o programa em python rms.py. Como as bandas de PAH são mais intensas para comprimentos de onda menores no infravermelho médio, dois tipos de RMS foram selecionados - um total, com todo o espectro de cada alvo, e um parcial, somente para comprimentos de onda menores que $12 \mu \mathrm{m}$. Assim, esta região poderia ser melhor analisada.

$$
x_{r m s}=\sqrt{\frac{1}{N} \sum_{i=o}^{N} x_{i}^{2}}
$$

\subsubsection{Resultados}

Abaixo, estão dispostas algumas figuras feitas com o PAHFIT. As demais, juntamente com os gráficos das subtrações com os respectivos RMS, podem ser vistas no Apêndice B. Por serem muitos gráficos, nem todos foram adicionados à dissertação. Porém, a Tabela D.2 contém os valores parciais e totais obtidos para cada uma das galáxias estudadas. As figuras à esquerda são os ajustes produzidos pela rotina PAHFIT (cores explicadas na Tabela 3.1). À direita, são seus gráficos de RMS absolutos do melhor ajuste (linha rosa).

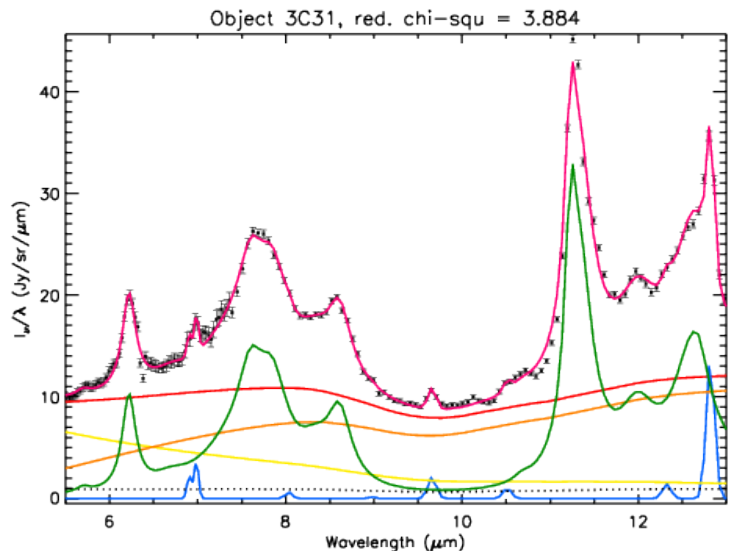

Figura 3.1: Espectro do objeto 3C31 decomposto com o PAHFIT e o valor do $\chi^{2}$ reduzido.

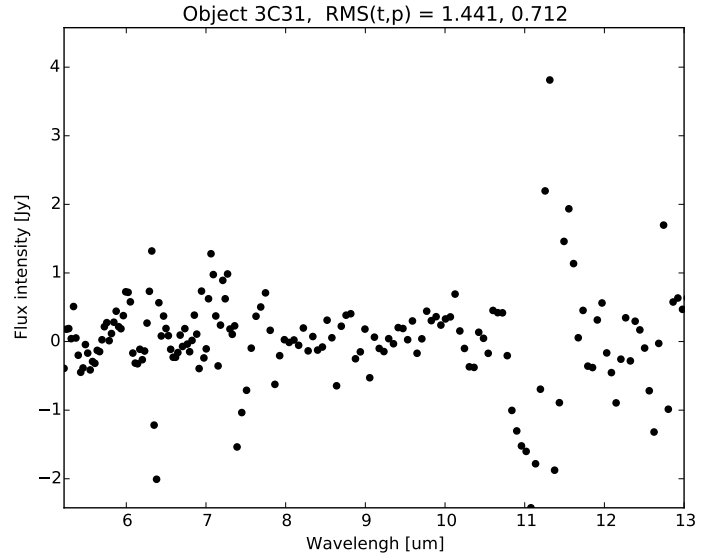

Figura 3.2: Gráfico de RMS do objeto 3C31 com os valores total e parcial. 


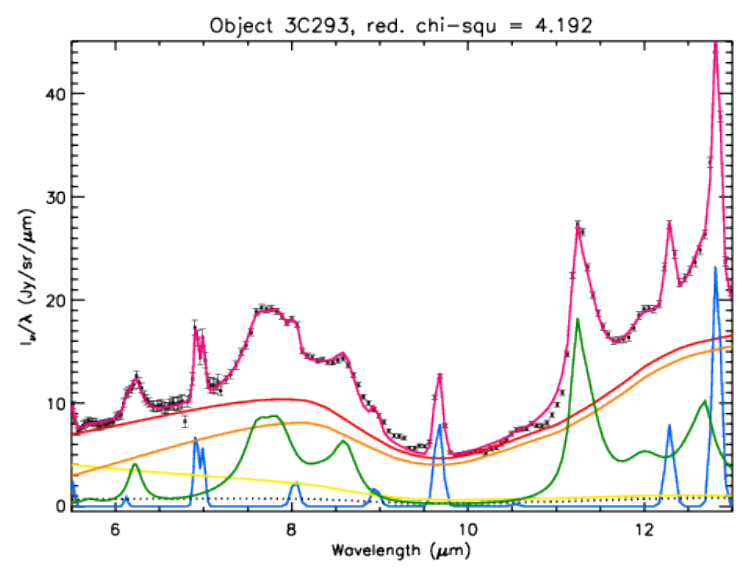

Figura 3.3: Espectro do objeto $3 C 293$ decomposto com o PAHFIT e o valor do $\chi^{2}$ reduzido.

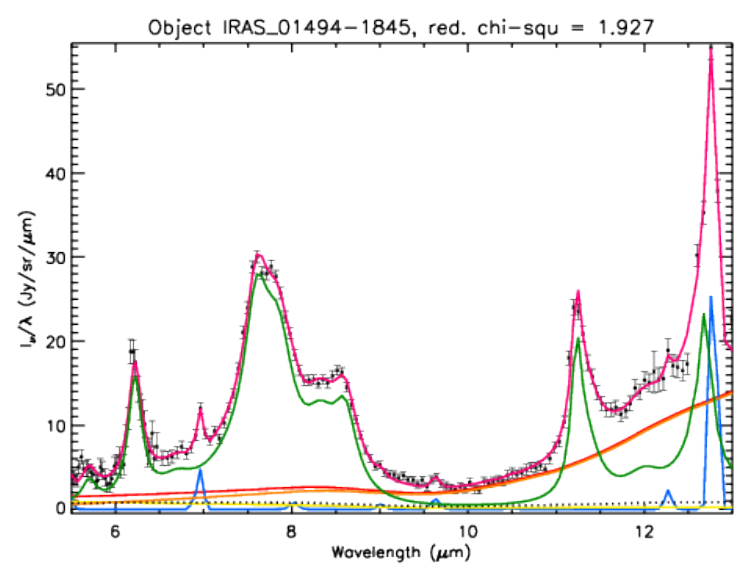

Figura 3.5: Espectro do objeto IRAS_01494-1845 decomposto com o PAHFIT e o valor do $\chi^{2}$ reduzido.

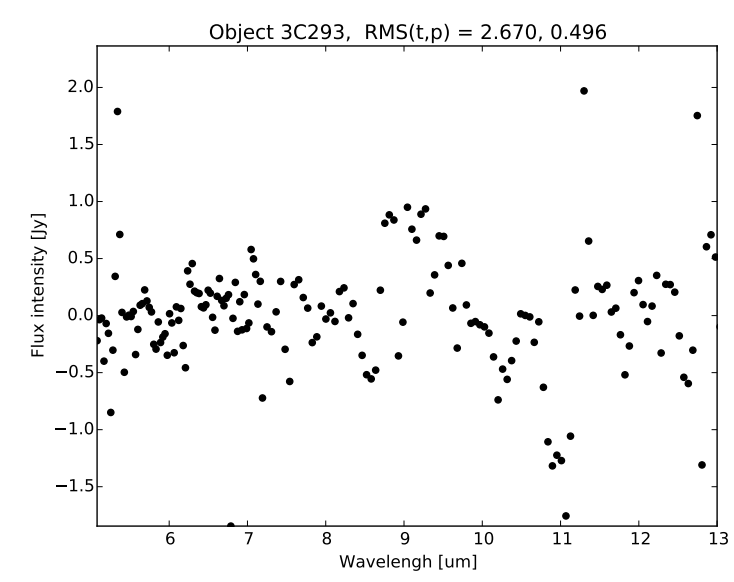

Figura 3.4: Gráfico de RMS do objeto 3C293 com os valores total e parcial.

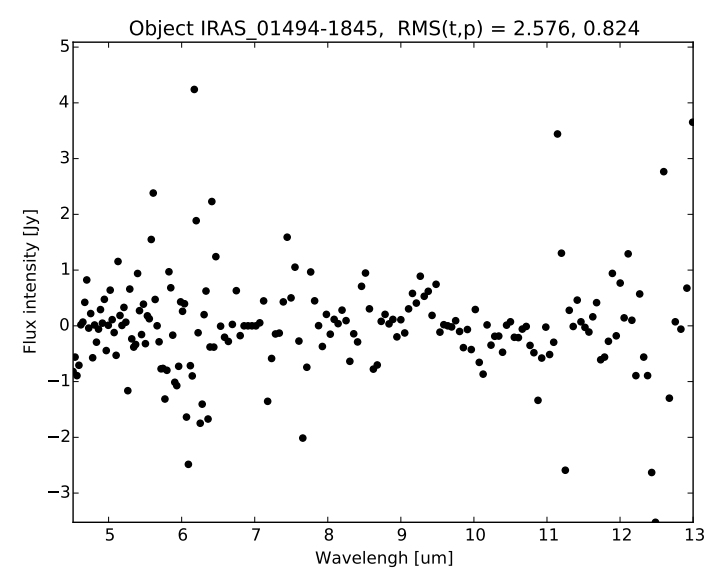

Figura 3.6: Gráfico de RMS do objeto IRAS_01494-1845 com os valores total e parcial. 


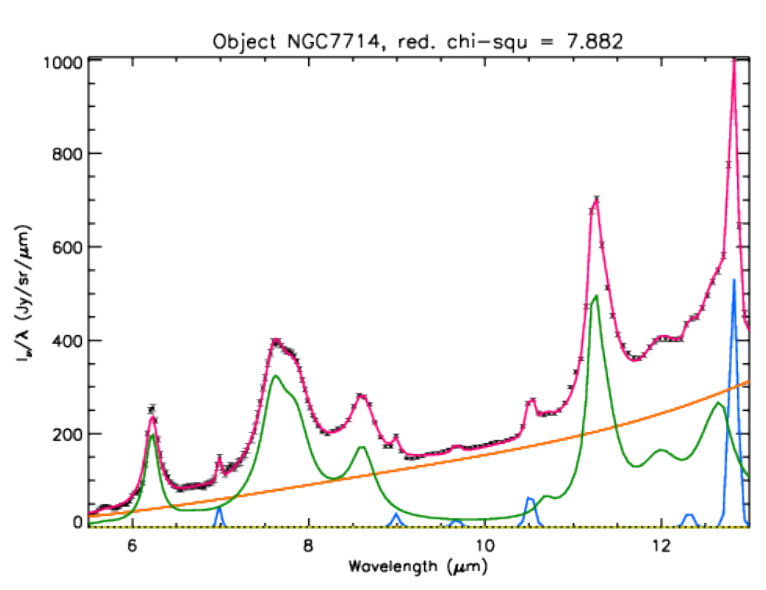

Figura 3.7: Espectro do objeto NGC7714 decomposto com o PAHFIT e o valor do $\chi^{2}$ reduzido.

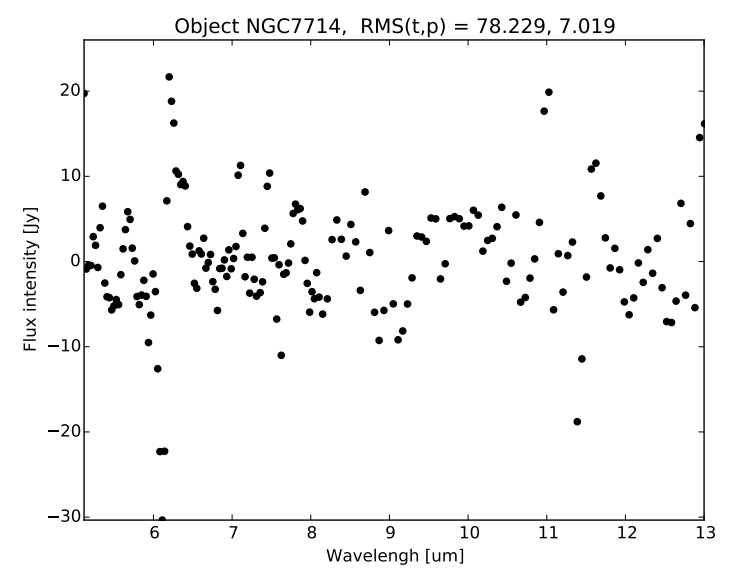

Figura 3.8: Gráfico de RMS do objeto NGC7714 com os valores total e parcial.

Quase todos os valores parciais são menores que os totais, como mostrado pelos gráficos de RMS acima, o que pode ser um efeito da ineficiência do PAHFIT em regiões do espectro com poucas emissões de poeira. Para NGC7714 (Figuras 3.7 e 3.8), esta diferença é enorme. Então, focando-se nos RMS parciais, foram feitas as Figuras 3.9 e 3.10 com o auxílio do programa merge1.py, mostrando suas dependências com os redshifts das galáxias.

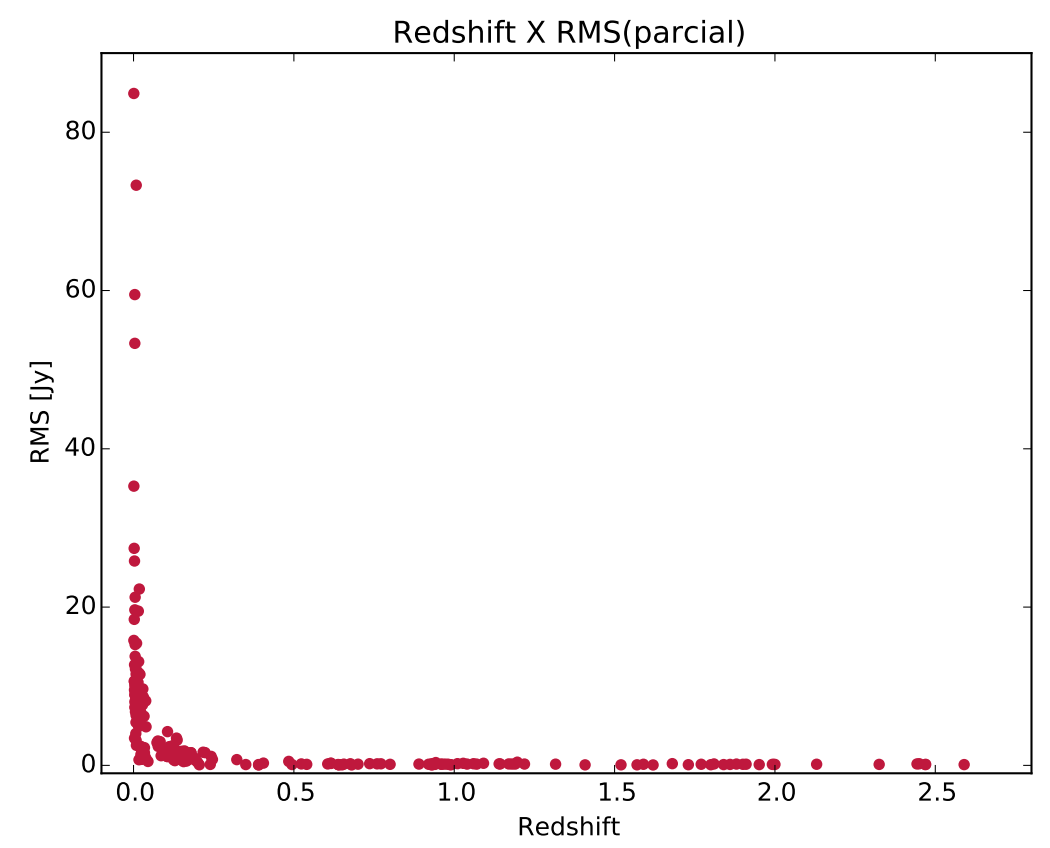

Figura 3.9: Gráfico do valor parcial absoluto de RMS para as fontes por seus respectivos redshifts. 


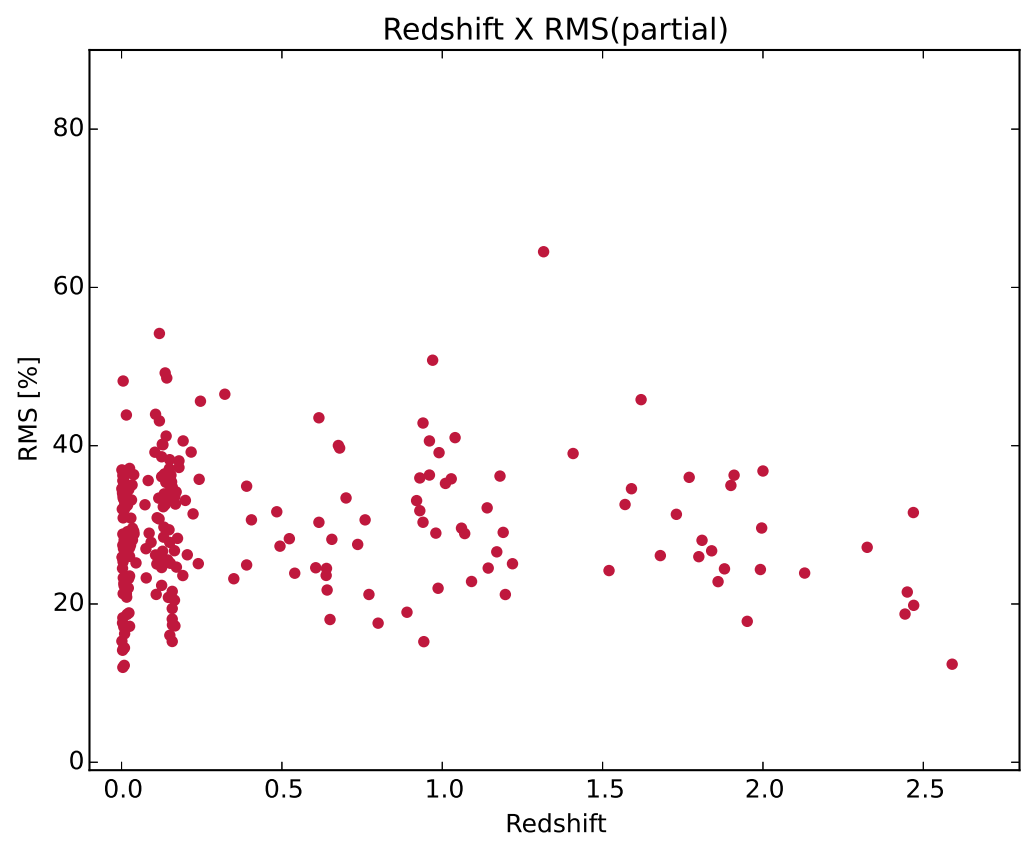

Figura 3.10: Gráfico do valor parcial normalizado de RMS para as fontes por seus respectivos redshifts.

Na Figura 3.9, os valores RMS são absolutos e, para uma análise geral da situação, é necessário normalizá-los. Assim, a Figura 3.10 mostra a porcentagem de cada RMS em relação ao fluxo máximo parcial de cada objeto. Ou seja, o fluxo máximo usado para normalizar o valor de RMS de cada espectro foi extraído dos comprimentos de onda menores do que $12 \mu m$ (referente ao intervalo estudado aqui). Os resultados estão entre 10 e $70 \%$, sendo que os mais elevados podem revelar uma complexidade nas bandas de PAHs e diferenças espectrais entre as diversas fontes, mais facilmente detectadas por causa da melhor resolução relacionada com a proximidade das mesmas. Realmente, maiores valores ocorreram para redshifts menores. Um olhar amplo pode sugerir cautela ao se optar pelo PAHFIT. Afinal, embora a maioria esteja abaixo dos 40\%, esta taxa ainda indica certa ineficiência no ajuste espectral que pode interferir nos resultados finais e análises. Um possível responsável seria, por exemplo, manter as posições de comprimento de onda central de PAHs fixas, como já abordado. Contudo, de modo geral, pode-se confiar nos ajustes obtidos e usá-los nas próximas etapas, especialmente se levando em conta que este trabalho é estatístico. 


\subsection{A banda de $6.2 \mu m$}

Antes de se investigar os perfis de PAHs, é preciso retirar as contribuições do contínuo, da absorção do silicato e das emissões iônicas e moleculares, já estimadas pelo PAHFIT. Este procedimento foi efetuado pelo programa em IDL substract_components.pro. Em seguida, foi elaborado outro programa, em python, para se determinar a posição central do pico da banda de $6.2 \mu m$ - o pah62_curvefit.py. Ele incorpora a ferramenta curve_fit do pacote scipy.optmize que utiliza mínimos quadrados não lineares para ajustar uma função aos dados, retornando, inclusive, os desvios padrões 2 . A função escolhida foi o perfil de Lorentz, por descrever melhor as asas desta banda do que uma Gaussiana e um perfil de Drude (mais adequado para asas mais largas, como abordado anteriormente). A Equação 3.3 mostra a fórmula do perfil, onde $\lambda_{r}$ é o comprimento de onda central, $\gamma$ é a FWHM e $b_{r}$ é a intensidade central. Os palpites iniciais de $\lambda_{i}=6.22 \mu \mathrm{m}$ para o comprimento de onda central e de $\gamma_{i}=0.187$ para FWHM foram extraídos de Smith et al. (2007). O intervalo padrão de comprimento de onda considerado para o ajuste foi de $6.07 \leq \lambda \leq 6.43$.

$$
I_{\nu}^{r}=\frac{b_{r}}{\pi} \times \frac{\frac{\gamma}{2}}{\left(\lambda-\lambda_{r}\right)^{2}+\left(\frac{\gamma}{2}\right)^{2}}
$$

Todavia, durante o processo, foi constatada a presença de outra banda de emissão perto de $6.35 \mu \mathrm{m}$ em algumas fontes. Por este motivo, resolveu-se adicionar um outro perfil após o de Lorentz, para melhor descrever a situação. Como a banda de $6.2 \mu m$ apresenta uma cauda mais alongada para o vermelho (Peeters et al., 2002), optou-se por um perfil de Drude. Infelizmente, o curve_fit não oferece muito controle nos parâmetros para mais de uma função ajustada. E, assim, deu-se preferência para a ferramenta minimize com o método o SLSQP (sigla em inglês para programação sequencial de mínimos quadrados), também do pacote scipy.optmize, introduzida na outra versão do programa - pah62_opt2.py. Ela permite obrigar que o pico do perfil de Drude seja depois do de Lorentz e ainda restringir os intervalos das variáveis $3^{3}$. Como ela não propaga as incertezas, foram inseridas rotinas para medir os valores de RMS e de $\chi^{2}$ reduzido. Para comparar os resultados futuramente,

\footnotetext{
${ }^{2}$ http://docs.scipy.org/doc/scipy-0.14.0/reference/generated/scipy.optimize.curve_fit.html, acessado em Fevereiro/2016

3 http://docs.scipy.org/doc/scipy/reference/generated/scipy.optimize.minimize.html\#scipy.optimize.minimize, acessado em Fevereiro/2016
} 
estas rotinas também foram acrescentadas ao pah62_curvefit.py.

Além disso, para avaliar qual o melhor método para cada galáxia, ambos foram rodados e o menor valor de RMS e de $\chi^{2}$ reduzido selecionou o resultado mais indicado. Todos os ajustes obtidos a partir do pah62_curvefit.py estão em vermelho, enquanto que todos os do pah62_opt2.py estão em roxo.

Os palpites iniciais para a primeira banda foram mantidos, mas se acrescentou valores mínimo e máximo para o comprimento de onda central $-6.18 \leq \lambda_{i 1} \leq 6.31$. Para a segunda, $\lambda_{i 2}=6.35$ e $\gamma_{i 2}=0.45$ também foram retirados de Smith et al. (2007) e se adotou $6.33 \leq \lambda_{i 2} \leq 6.45$. O intervalo padrão de comprimento de onda considerado para o ajuste foi de $6.07 \leq \lambda \leq 6.50$. Infelizmente, para muitos alvos, algumas destas considerações não estavam de acordo com os dados. Basicamente, os intervalos deveriam cobrir somente o início e o fim da(s) banda(s). Porém, se outros aspectos do espectro estiverem contidos neles, o ajuste final poderia ser prejudicado. Portanto, eles tiveram que ser corrigidos um a um.

No caso em que somente um perfil foi empregado (pah62_curvefit.py), o pico da banda ajustada estava claramente desviado para o vermelho por causa da mesma cauda vermelha própria da banda. Para resolver esta questão, esta cauda não foi incluída. Os novos intervalos variaram entre as galáxias e estão descritos nas respectivas imagens de cada uma.

Com as posições centrais dos picos adquiridas, a catalogação entre as classes de Peeters (intervalos utilizados na Tabela 3.2) e os gráficos com a comparação dos resultados (Figuras 3.26 a 3.29 foram possíveis graças aos programas merge2.py e merge3.py, respectivos ao pah62_curvefit.py e ao pah62_opt2.py.

Tabela 3.2 - Intervalo para cada classe de Peeter (Peeters et al. 2002).

\begin{tabular}{cc}
\hline \hline Classe & Intervalo $(\mu m)$ \\
\hline A & $<6.23$ \\
B & $6.23<\lambda<6.29$ \\
C & $>6.29$ \\
& \\
\hline
\end{tabular}




\subsubsection{Resultados}

Das 229 galáxias selecionadas, 206 (90\%) tiveram a banda de $6.2 \mu m$ averiguada. As demais, ou os programas não conseguiram ajustar os dados, ou simplesmente as galáxias não tinham esta região do espectro observada. As Tabelas D.3 e D.4 referem-se aos resultados dos programas para todas as fontes. Os objetos com a banda puderam ser divididos em quatro grupos - os melhor ajustados pela função curve_fit (61 fontes - 29.6\%), os melhor ajustados pela minimize (108 fontes - 52.4\%), os que apresentaram pouca diferença entre os programas e/ou uma característica distinta ( 8 fontes $-4 \%$ ), e aqueles cujos resultados são inconclusivos (29 fontes - 14\%). Eles são discutidos, separadamente, a seguir.

Em algumas galáxias, foi percebida a presença de um perfil de absorção perto de $6.0 \mu m$ devido ao gelo d'água. Embora ele interfera muito pouco com a banda de $6.2 \mu m$ dos PAHs (Spoon et al., 2002), é interessante de ser mencionado (Seção 3.3).

\subsubsection{Melhor ajuste pelo pah62_curvefit.py}

Algumas fontes foram selecionadas para exemplificar os resultados. Por causa da quantidade de gráficos, somente uma porção está exposta a seguir e os resto está no Apêndice C.1.

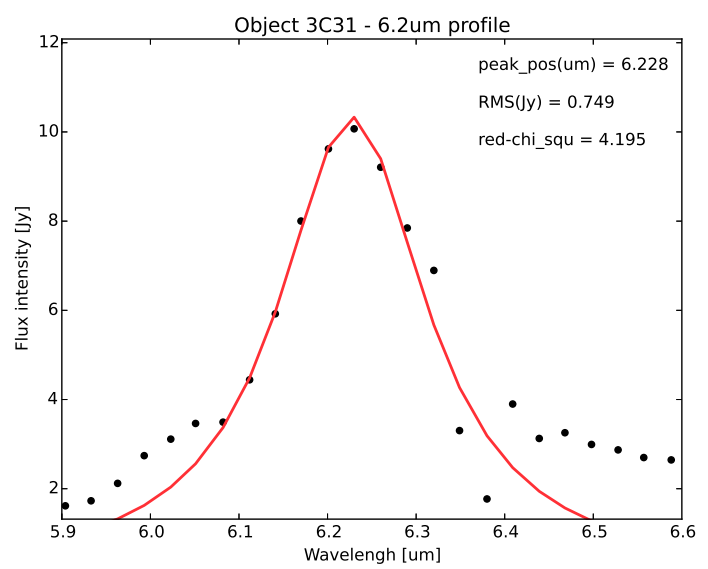

Figura 3.11: Peril da banda de $6.2 \mu m$ do objeto 3C31 ajustado com curve_fit.

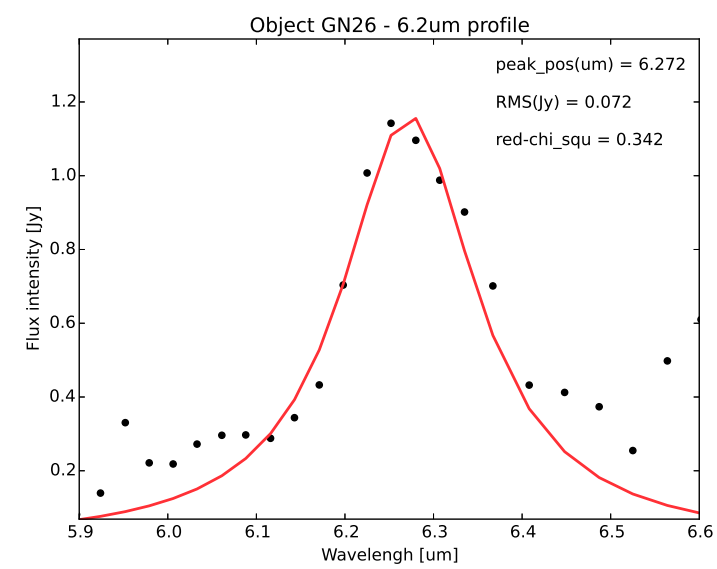

Figura 3.12: Perfil da banda de $6.2 \mu m$ do objeto GN26 ajustado com curve_fit. 


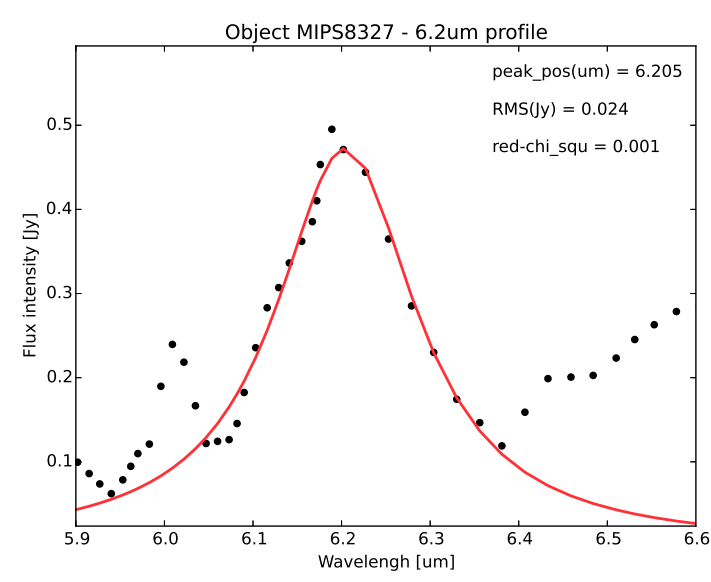

Figura 3.13: Perfil da banda de $6.2 \mu m$ do objeto MIPS8327 ajustado com curve_fit.

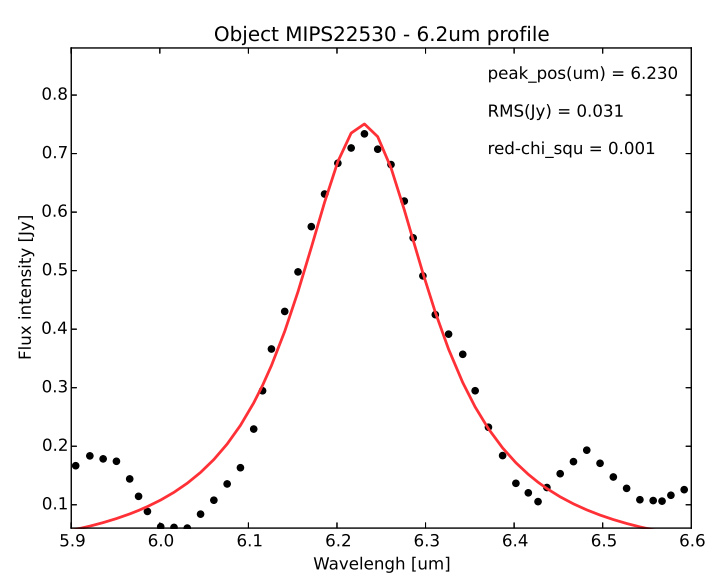

Figura 3.14: Perfil da banda de $6.2 \mu \mathrm{m}$ do objeto MIPS22530 ajustado com curve_fit.

Para algumas galáxias, o perfil assimétrico é bem evidente. A cauda vermelha precisou ser desconsiderada em certas ocasiões para se ajustar o centro do pico, como na Figura 3.15. A Figura 3.16 também externa esta característica desta banda.

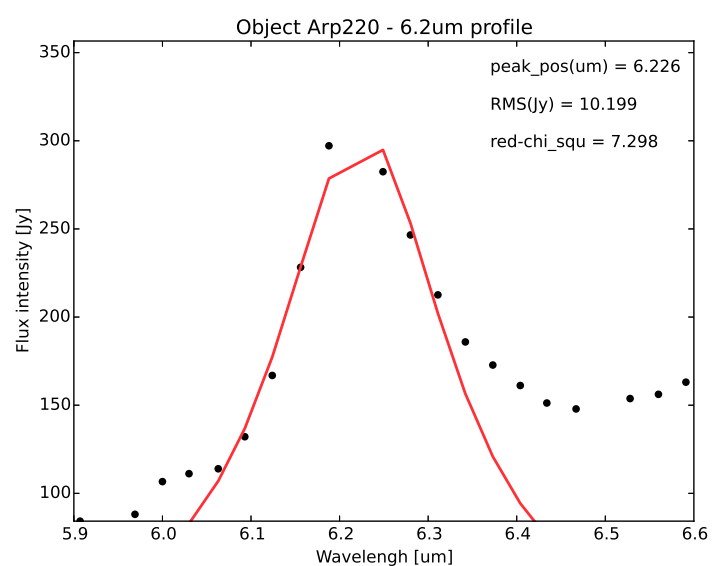

Figura 3.15: Perfil da banda de $6.2 \mu m$ do objeto Arp220 ajustado com curve_fit e para $6.05 \leq \lambda \leq 6.32$.

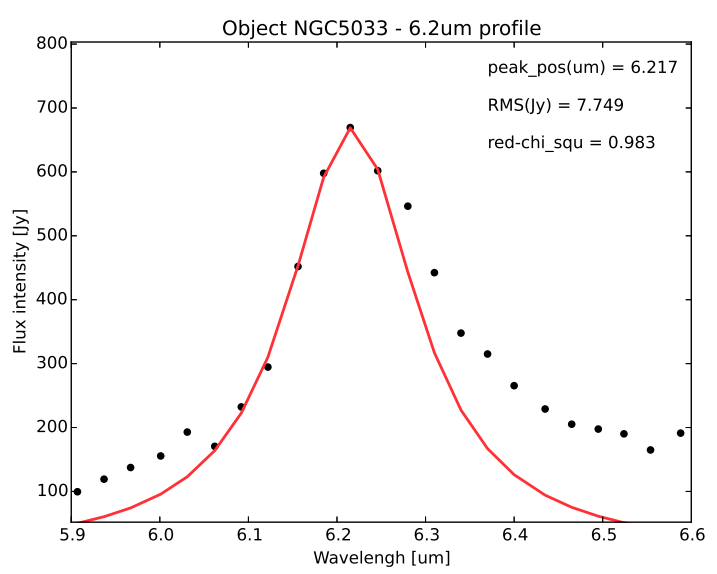

Figura 3.16: Perfil da banda de $6.2 \mu m$ do objeto NGC5033 ajustado com curve_fit e para $6.05 \leq$ $\lambda \leq 6.26$.

O objeto MIPS180 se mostrou ser um exemplar da classe C. Não só pela posição central do pico, mas também pelo formato do perfil. Além de ser mais simétrico do que os anteriores, possui asas e FWHM mais alargadas. Estes fatos podem indicar a presença de grãos de poeira - mais especificamente, VSG (sigla em inglês para grãos muito pequenos) - dominando a emissão da galáxia nesta região espectral. A evaporação de VSGs por radiação UV foi sugerida como um caminho para a formação de PAHs (Pilleri et al., 2012). 


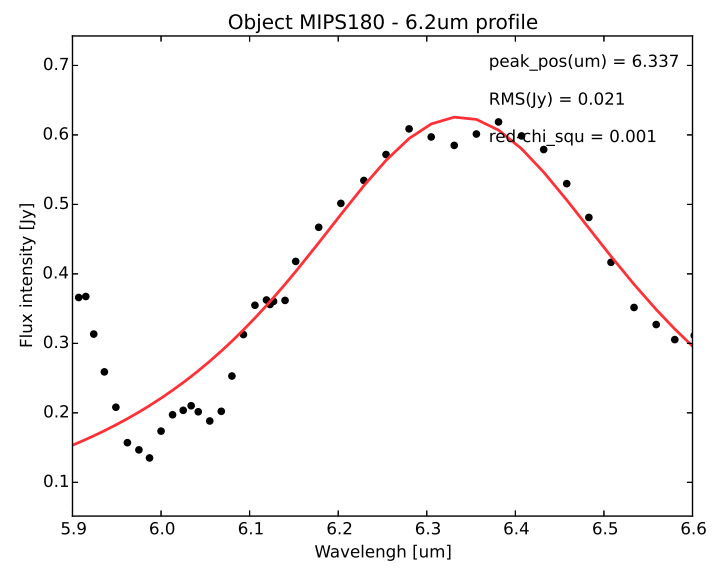

Figura 3.17: Perfil da banda de $6.2 \mu m$ do objeto MIPS180 ajustado com curve_fit.

\subsubsection{Melhor ajuste pelo pah62_opt2.py}

Novamente, somente algumas galáxias foram selecionadas (Figuras 3.18 a 3.21 ) e parte delas também está localizada no Apêndice C.2.

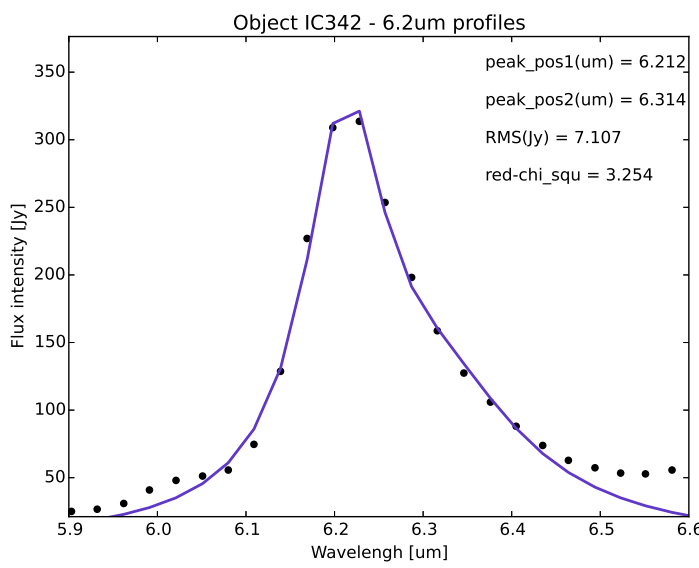

Figura 3.18: Perfil da banda de $6.2 \mu m$ do objeto IC342 ajustado com minimize e para $6.04 \leq$ $\lambda \leq 6.46,6.10 \leq \lambda_{i 1} \leq 6.25$ e $6.26 \leq \lambda_{i 2} \leq 6.40$.

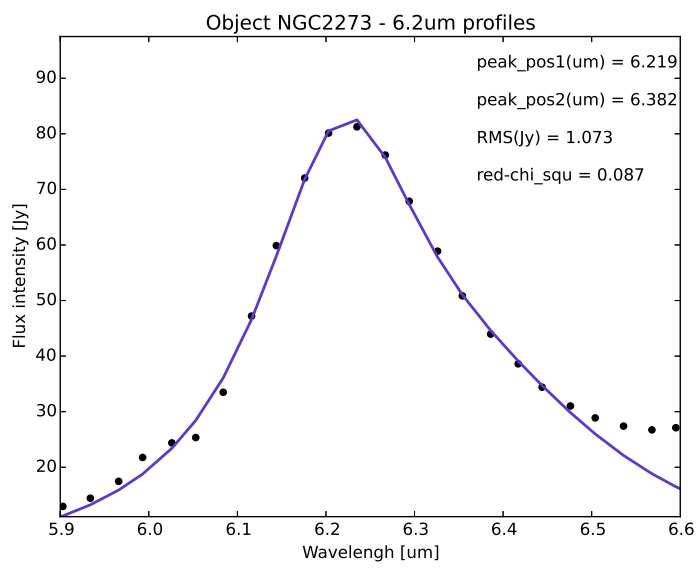

Figura 3.19: Perfil da banda de $6.2 \mu m$ do objeto NGC2273 ajustado com minimize.

Deve-se ressaltar que o perfil desta banda não é harmônico, não sendo exatamente bem descrito por uma Lorentziana, e suposições de presença de outras subcaracterísticas podem não ser reais (Tielens, 2008). Realmente, é difícil de se afirmar a veracidade desta segunda característica, que variou bastante de comprimento de onda central - de 6.246 a 6.471. Esta é uma diferença de 0.225 entre o mínimo e o máximo encontrado. Em contrapartida, a primeira variou de 6.170 a 6.259, o que corresponde à uma diferença de $0.089,39,5 \%$ 
menor. Contudo, ficou muito claro que a inclusão desta segunda emissão descreve melhor o perfil (de $6.2 \mu m)$ em si e a sua respectiva posição de pico, mesmo se a característica não for verdadeira. Isto pode ser visualizado nos próprios gráficos acima.

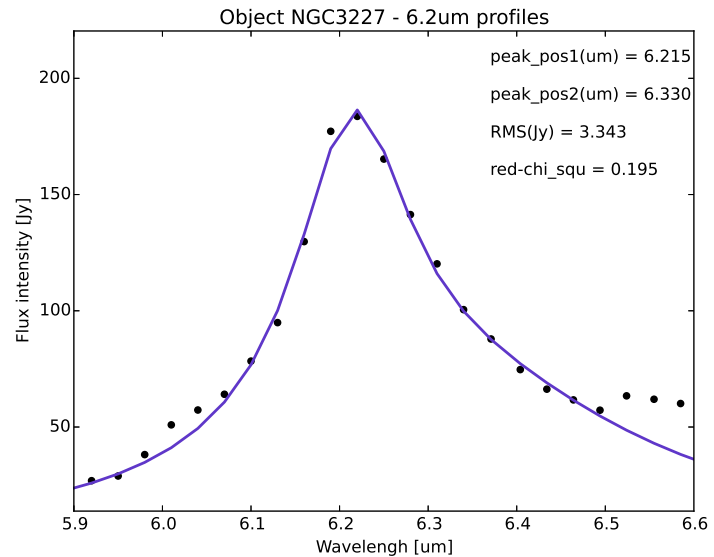

Figura 3.20: Perfil da banda de $6.2 \mu m$ do objeto NGC3227 ajustado com minimize.

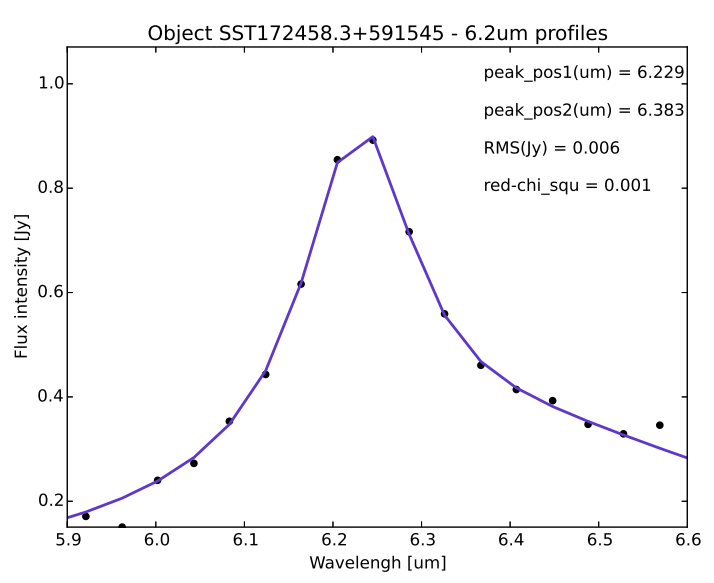

Figura 3.21: Perfil da banda de $6.2 \mu m$ do objeto SST172458.3+591545 ajustado com minimize.

Mas, algumas fontes aparentam possuir duas bandas distintas, como as abaixo. Segundo Pino et al. (2008), que realizou experimentos com PAHs para verificar a origem de suas emissões, bandas em $6.3 \mu m$ estão ligadas à características alifáticas. No entanto, a banda em $6.4 \mu m$ observada na nebulosa de reflexão NGC7023 foi atribuída ao cátion $C_{60}^{+}$Berné et al. 2015).

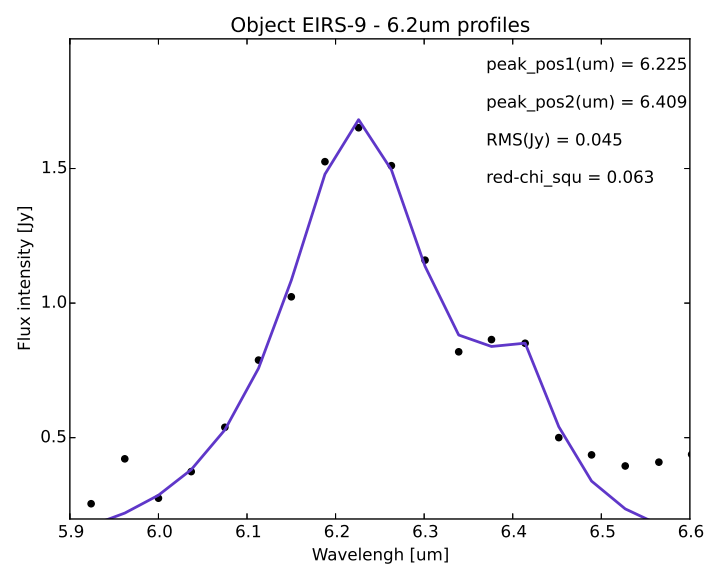

Figura 3.22: Perfil da banda de $6.2 \mu m$ do objeto EIRS-9 ajustado com minimize.

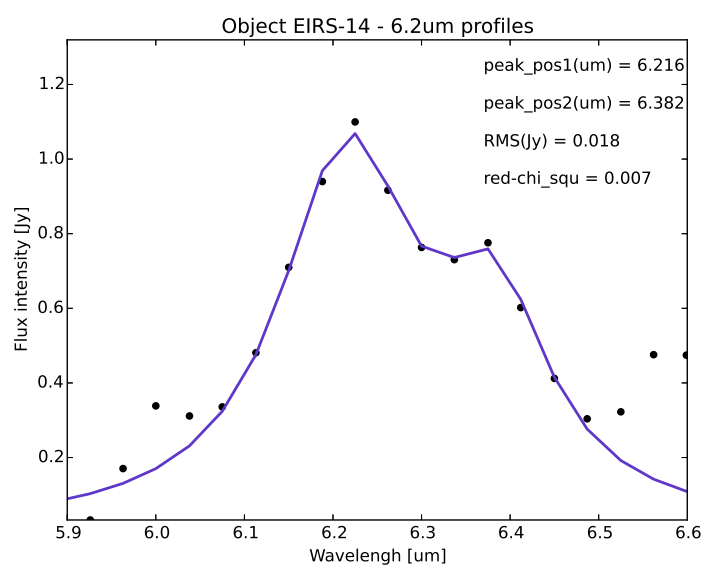

Figura 3.23: Perfil da banda de $6.2 \mu m$ do objeto EIRS-14 ajustado com minimize. 


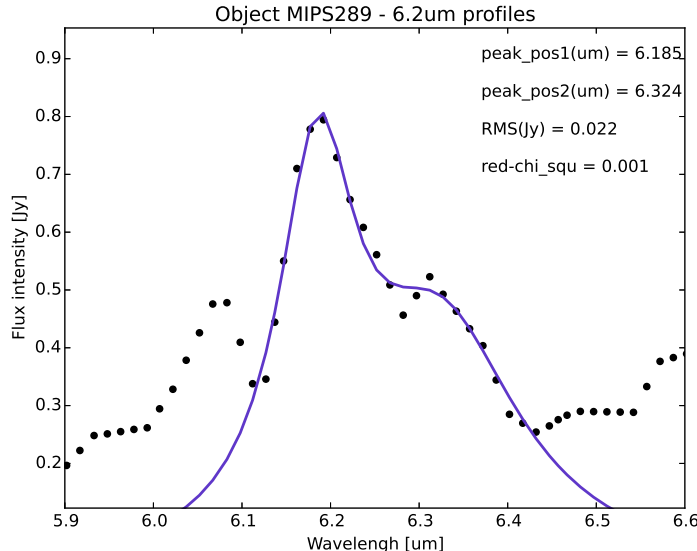

Figura 3.24: Perfil da banda de $6.2 \mu \mathrm{m}$ do objeto MIPS289 ajustado com minimize e para $6.105 \leq \lambda \leq 6.40,6.1 \leq \lambda_{i 1} \leq 6.2,6.29 \leq$ $\lambda_{i 2} \leq 6.34, \lambda_{i 1}=6.17$ e $\lambda_{i 2}=6.31$.

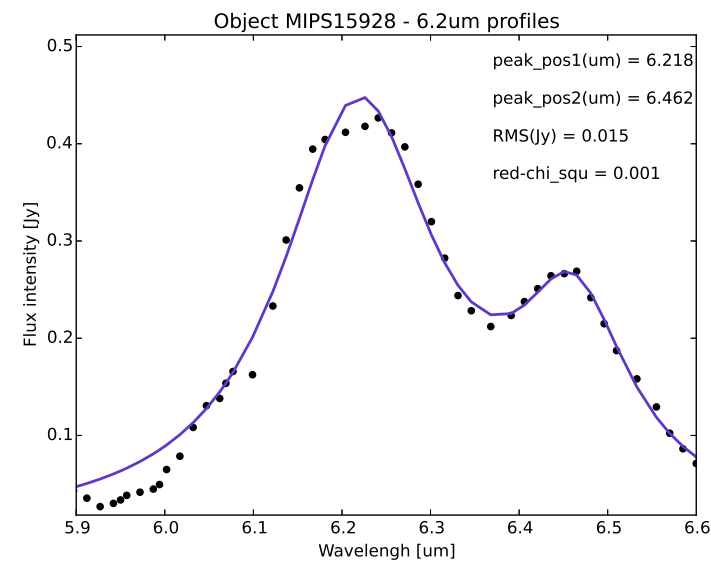

Figura 3.25: Perfil da banda de $6.2 \mu \mathrm{m}$ do objeto MIPS15928 ajustado com minimize e para $6.0 \leq$ $\lambda \leq 6.6,6.1 \leq \lambda_{i 1} \leq 6.3,6.39 \leq \lambda_{i 2} \leq 6.50 \mathrm{e}$ $\lambda_{i 2}=6.45$.

\subsubsection{Visão geral dos resutados}

A Tabela 3.3 abaixo mostra a distribuição das galáxias entre as classes de Peeters, montada com os resultados descritos anteriormente. Um resumo contendo a posição de pico e sua incerteza, o tipo de galáxia, sua classe de Peeters e seu redshift de todos os objetos considerados bem ajustados encontra-se na Tabela D.5. Os casos que são discutidos nos próximos itens não entraram para esta estatística.

Tabela 3.3 - Quantidade de galáxias categorizadas em cada classe de Peeters.

\begin{tabular}{lcccc}
\hline \hline Programa usado & Galáxias ajustadas & Classe A & Classe B & Classe C \\
\hline pah62_curvefit.py & 61 & 31 & 27 & 3 \\
pah62_opt2.py & 108 & 93 & 15 & 0 \\
Total & $\mathbf{1 6 9}$ & $\mathbf{1 2 4}$ & $\mathbf{4 2}$ & $\mathbf{3}$ \\
& & & & \\
\hline
\end{tabular}

Já se havia notado que objetos da classe A são os mais comuns no Universo e abrangem diversos tipos astrofísicos, enquanto que representantes da classe C são minoria (Pino et al. 2008). Nossos resultados apontam a mesma direção. E, para galáxias dominadass por starbursts, a dominação da classe A é enorme, como pode ser visto nas imagens a seguir. As figuras 3.26 a 3.28 exibem os resultados encontrados com o curve_fit. Igualmente, as 
figuras 3.29 e 3.30 referem-se aos resultados do minimize.

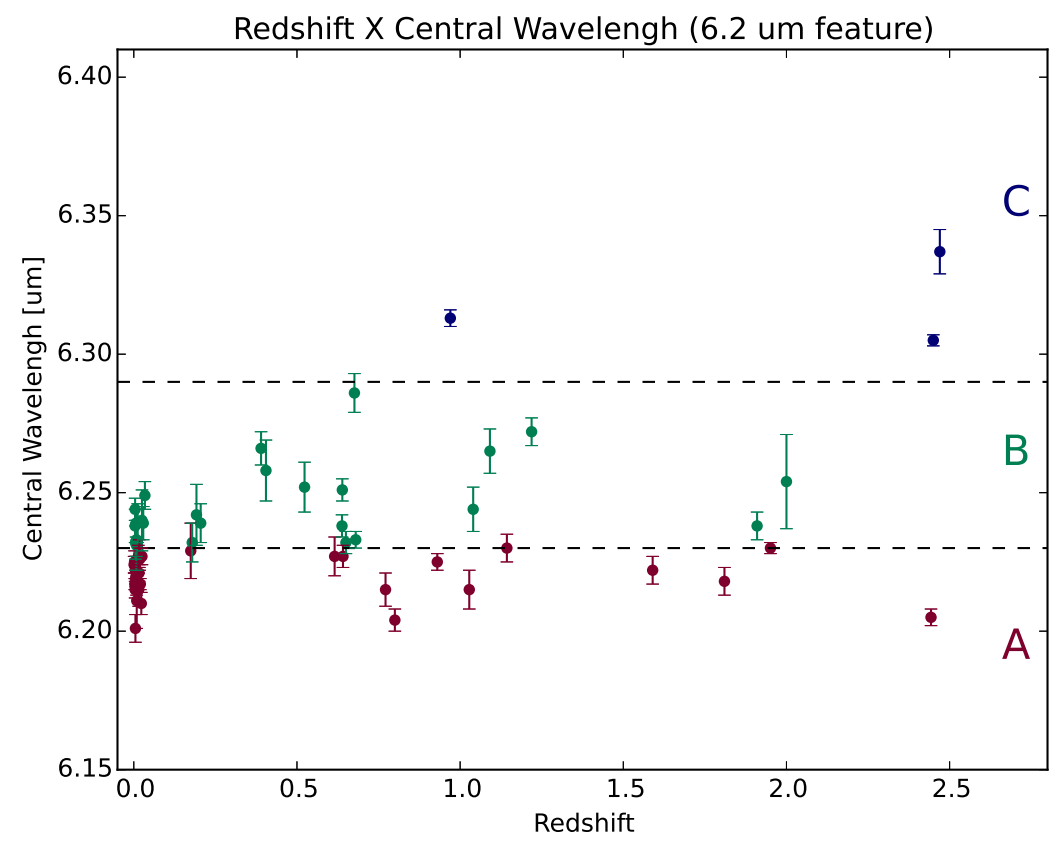

Figura 3.26: Gráfico do comprimento de onda central da banda de $6.2 \mu m$ ajustado com o pah62_curvefit.py, representados por pontos e suas respectivas barras verticais de incerteza, pelo redshift das fontes. As bandas tracejadas cinzas mostram o limite entre as três classes de Peeters. Classe A - cor de vinho; Classe B - verde; e Classe C - azul.

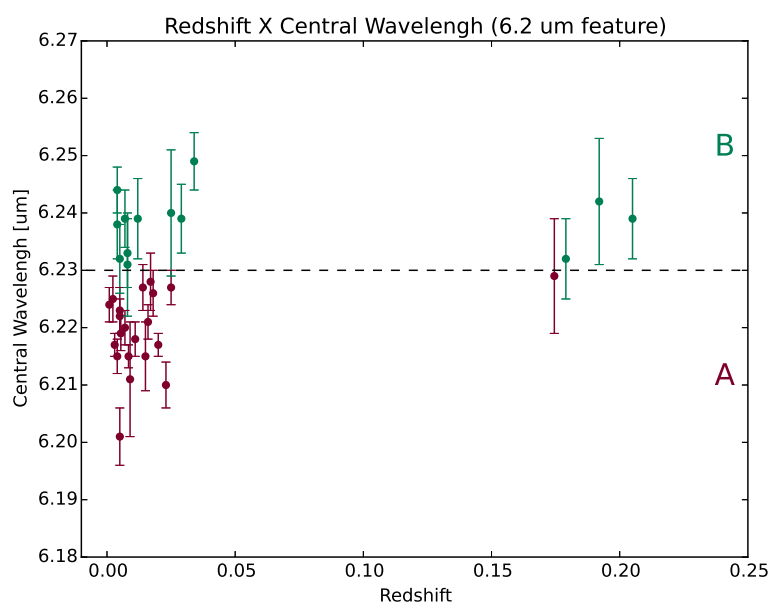

Figura 3.27: Recorte da Figura 3.26 com valores de redshift $<0.25$. A Classe $\mathrm{C}$ não possui representates para este intervalo de redshift.

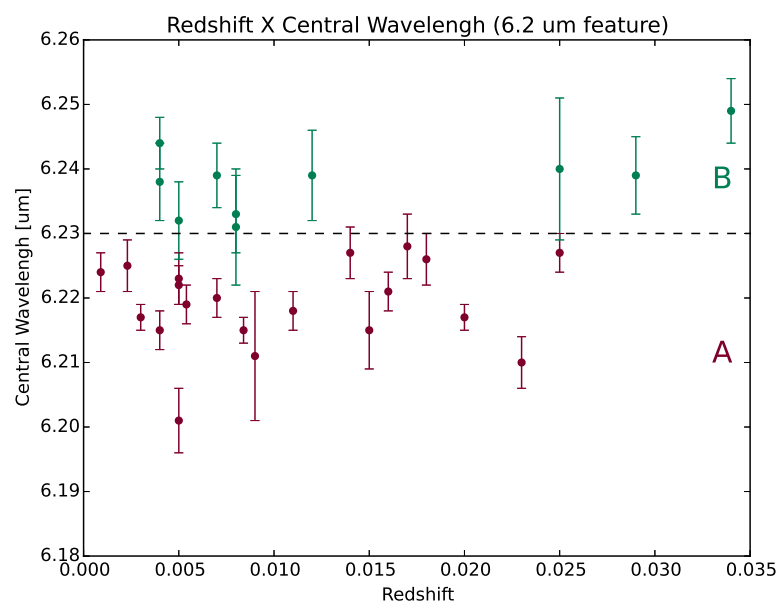

Figura 3.28: Recorte da Figura 3.26 com valores de redshift $<0.035$. A Classe $\mathrm{C}$ não possui representates para este intervalo de redshift. 


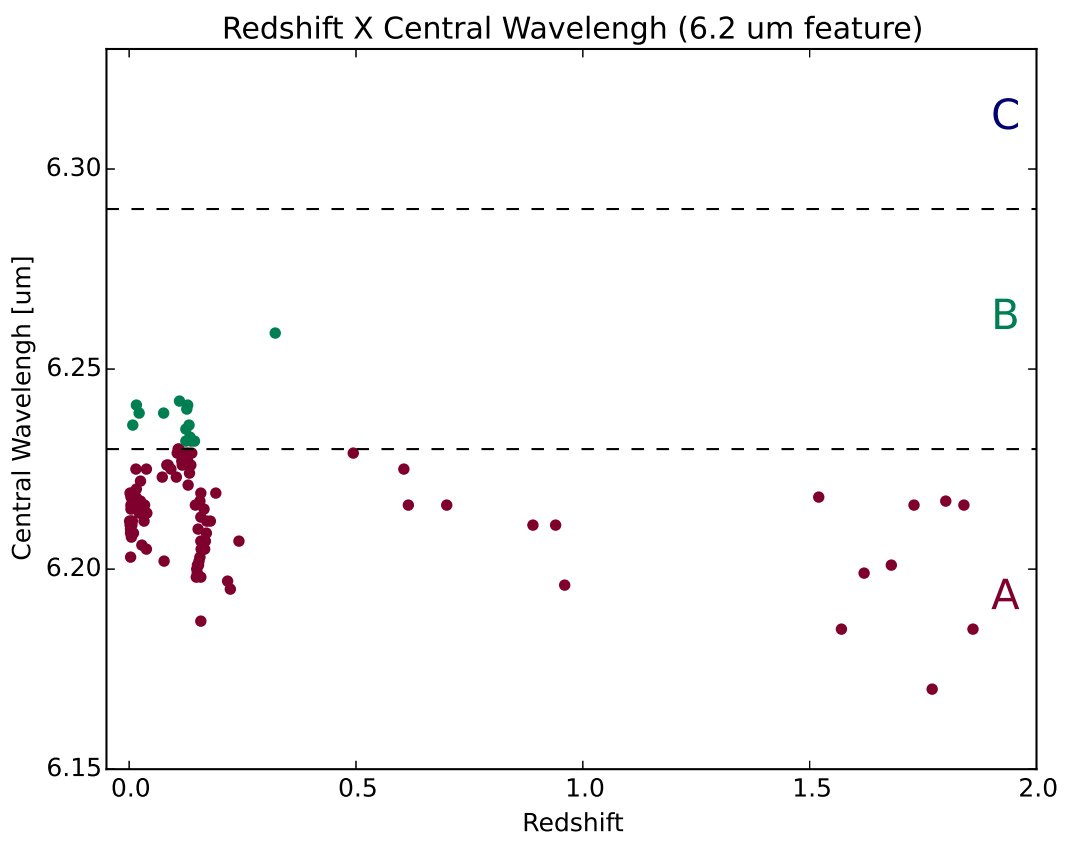

Figura 3.29: Gráfico do comprimento de onda central da banda de $6.2 \mu m$ ajustado com o pah62_opt2.py pelo redshift das fontes. As linhas tracejadas pretas mostram o limite entre as três classes de Peeters. Classe A - cor de vinho; Classe B - verde; e Classe C - azul, que não possui representantes.

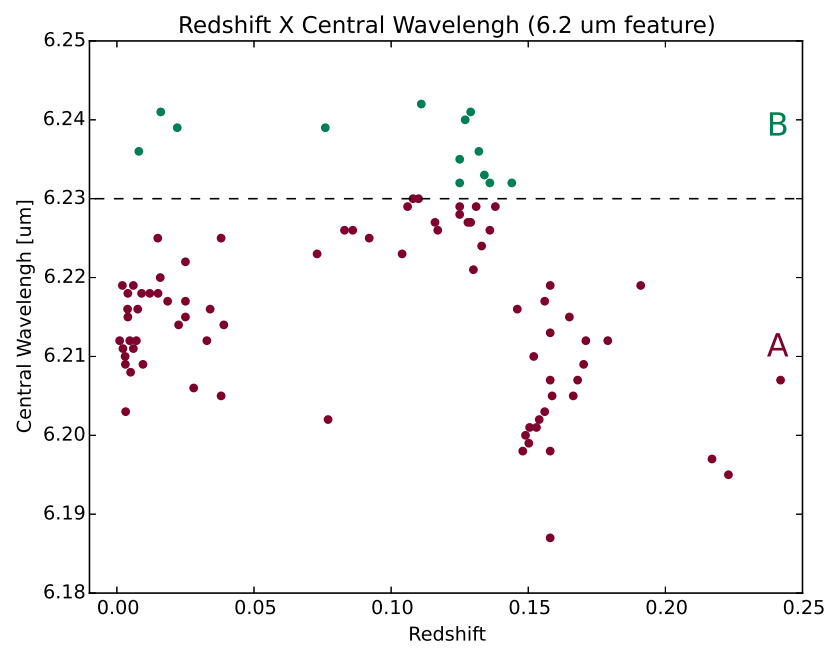

Figura 3.30: Recorte da Figura 3.29 com valores de redshifts $<0.25$.

Com somente três objetos da classe $\mathrm{C}$, a possibilidade de distinguir qualquer padrão para esta classe é baixa. Além do mais, existe uma galáxia classe A com $z \sim 2.5$. Assim, é complicado dizer, com certeza, que altos redshifts tendem a não conter PANHs. Em outras palavras, a ideia da representação da classe C por VSG e sua dominação a altos redshifts, o 
que implicaria numa escala de tempo evolutiva dos PAHs, não pode ser afirmada somente com estes resultados. Para uma visão mais aprimorada da situação, seriam necessárias observações de starbursts a redshifts mais elevados e em maior quantidade, possibilitando um trabalho estatístico mais amplo e completo.

Considerando-se redshifts menores, é muito clara a dominação de classe A em starbursts. E, levando-se em conta que a classe B pode ser uma mistura entre PANHs e PAHs, podese constatar que PANHs influenciam e dominam as emissões regiões de starbursts desta banda de $6.2 \mu m$.

De acordo com Ota (2016), a substituição de três ou mais átomos de nitrogênio nos PAHs não fornece moléculas que descrevam as características observadas. Isto indicaria que compostos de interesse biológico como purina e adenina talvez não possam ser sintetizados no ISM. Contudo, para um ou dois nitrogênios, foram encontradas espécies que conseguem recriar as observações $\left(C_{7} H_{5} N_{2}-a b^{3+}\right.$, por exemplo). Porém, somente pequenos PANHs foram considerados neste artigo e, quanto menor a estrutura aromática, mais facilmente ela é destruída no ISM.

Para uma visão mais específica destes resultados, a tabela e os gráficos abaixo mostram como a classificação das classes de Peeters varia com o tipo das galáxias. As classes de Peeters estão delimitadas pelas linhas tracejas pretas e indicadas pelas respectivas letras.

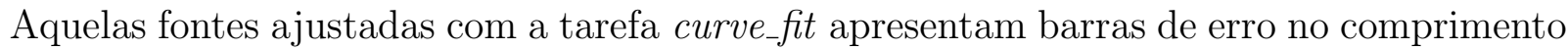
de onda. As demais, sem as incertezas, foram obtidas com o minimize. As siglas referem-se à AGN - Núcleo ativo de galáxia, FR - galáxia Fanaroff-Riley, HII - Região HII, IRgal galáxia no infravermelho, LINER - Regiões de emissão nuclear de baixa ionização, QSO - Objeto quasi-estelar, SB - galáxia Starburst, Sy -galáxia Seyfert, SMG - Galáxia no submilimétrico, e ULIRG - Galáxia no infravermelho ultra-luminosa.

Tabela 3.4 - Distribuição das classes de Peeters de acordo com o tipo de galáxia.

\begin{tabular}{lcccc}
\hline \hline Tipo & Total & Classe A & Classe B & Classe C \\
\hline AGN2 & 1 & 0 & 1 & 0 \\
FR-1 & 1 & 1 & 0 & 0 \\
IRgal & 3 & 2 & 1 & 0 \\
LINER & 1 & 0 & 1 & 0 \\
QSO & 1 & 0 & 1 & 0 \\
QSO2 & 1 & 0 & 1 & 0 \\
Continua na próxima página... & & &
\end{tabular}


Tabela 3.4 - Continuação

\begin{tabular}{lcccc}
\hline \hline Tipo & Total & Classe A & Classe B & Classe C \\
\hline SB & 22 & 18 & 4 & 0 \\
SB + HII & 4 & 4 & 0 & 0 \\
SB + Sy & 3 & 3 & 0 & 0 \\
SB + LINER & 1 & 1 & 0 & 0 \\
SMG & 6 & 2 & 4 & 0 \\
Sy1 & 10 & 9 & 1 & 0 \\
Sy intermediárias & 18 & 10 & 8 & 0 \\
Sy2 & 23 & 18 & 5 & 0 \\
ULIRG & 36 & 30 & 3 & 3 \\
ULIRG + HII & 17 & 10 & 7 & 0 \\
ULIRG + LINER & 21 & 16 & 5 & 0 \\
& & & & \\
\hline
\end{tabular}

Das 169 galáxias bem ajustadas, 43.8\% são ULIRGs (Figuras 3.31 e 3.32), 30\% são Seyferts (Figuras 3.33 e 3.34), 17.8\% são starbursts (Figuras 3.35 e 3.36) e os outros tipos somam $8.4 \%$ (Figura).

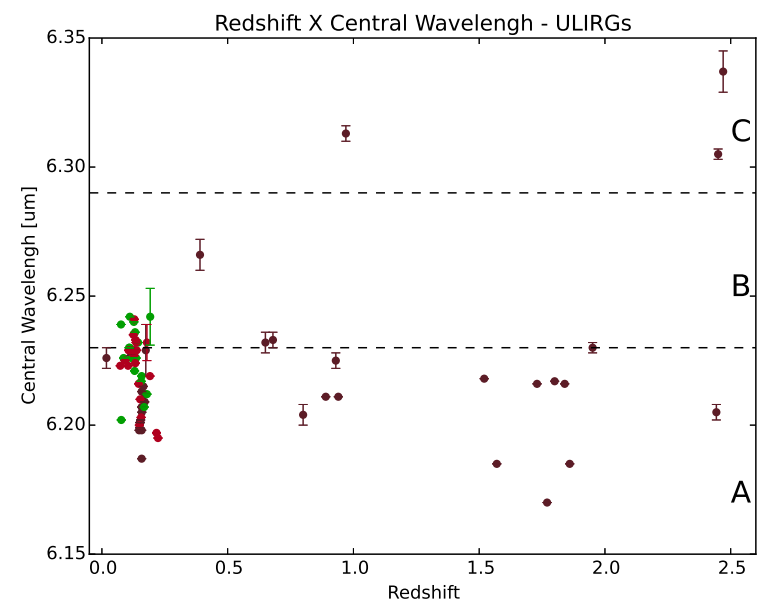

Figura 3.31: Gráfico do comprimento de onda central da banda de $6.2 \mu \mathrm{m}$ pelo redshift das ULIRGs. ULIRG - cor roxo escuro; ULIRG + LINER - cor rosa; e ULIRG + HII - cor verde.

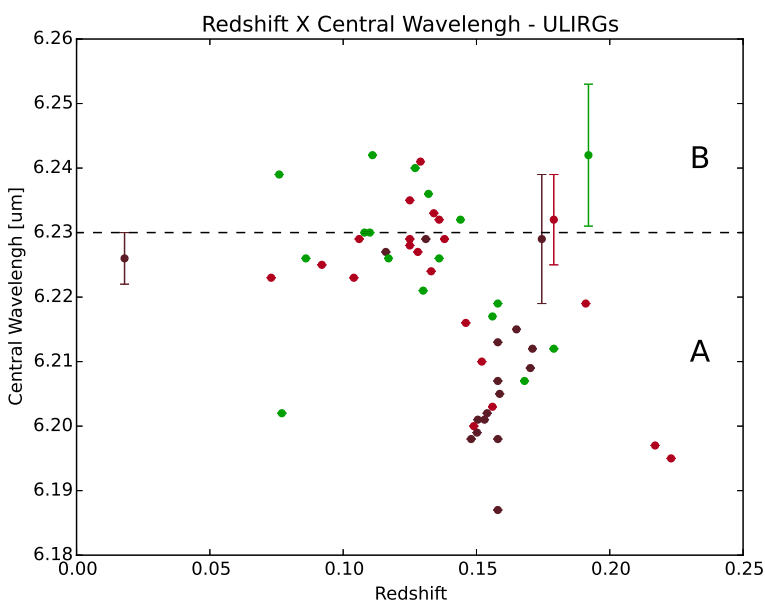

Figura 3.32: Recorte da Figura 3.31 com valores de redshift $<0.25$.

Somente galáxias no submilimétrico apresentaram uma dominação da classe B, com $67 \%$ de seus integrantes pertencendo à ela. Outro fato interessante é todos os objetos classe C serem ULIRGs. 


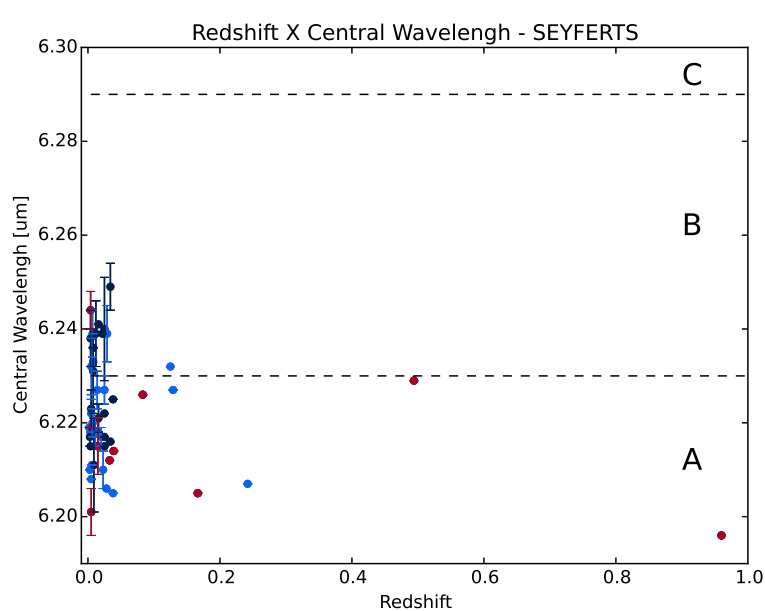

Figura 3.33: Gráfico do comprimento de onda central da banda de $6.2 \mu m$ pelo redshift das Seyferts. Sy1 - cor de vinho; Sy intermediária - cor azul escuro; e Sy2 - cor azul claro.

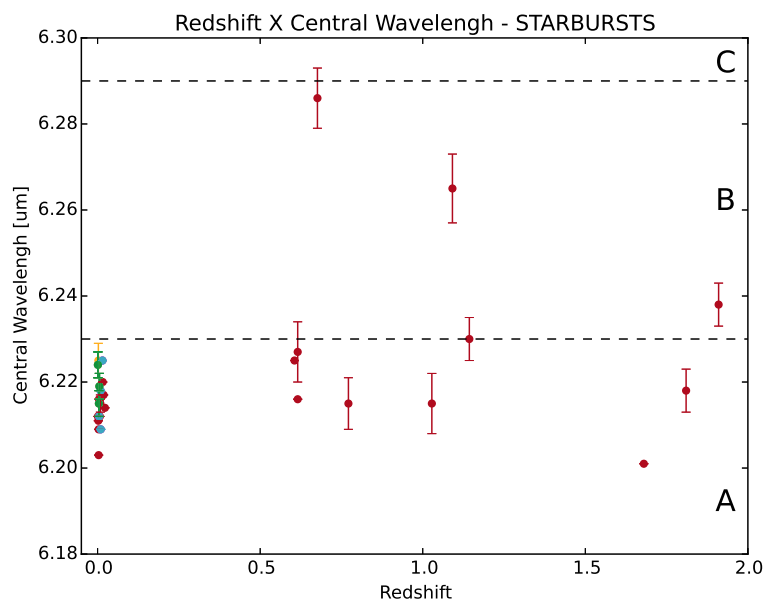

Figura 3.35: Gráfico do comprimento de onda central da banda de $6.2 \mu m$ pelo redshift das Starbursts. $\mathrm{SB}$ - cor de vinho; $\mathrm{SB}+\mathrm{HII}-$ cor azul claro; SB + Sy - cor verde; e SB + LINER - cor amarelo.

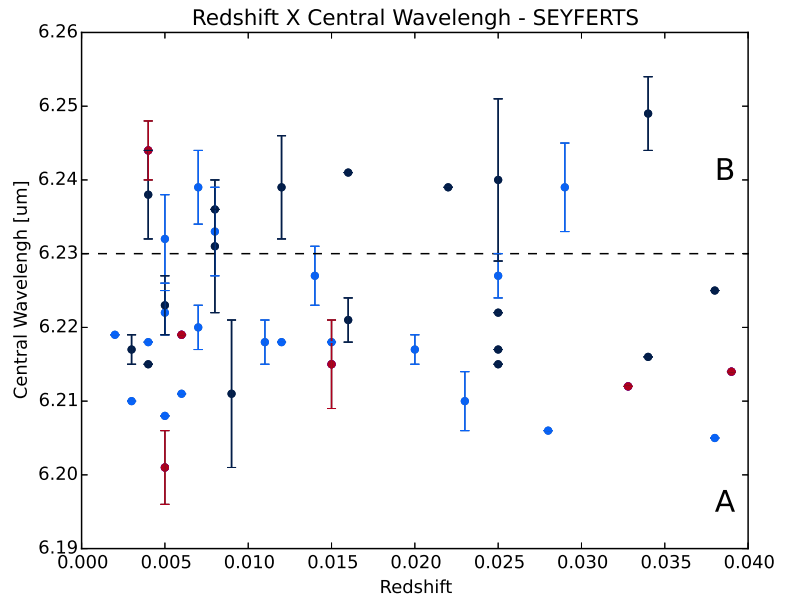

Figura 3.34: Recorte da Figura 3.33 com valores de redshift $<0.04$.

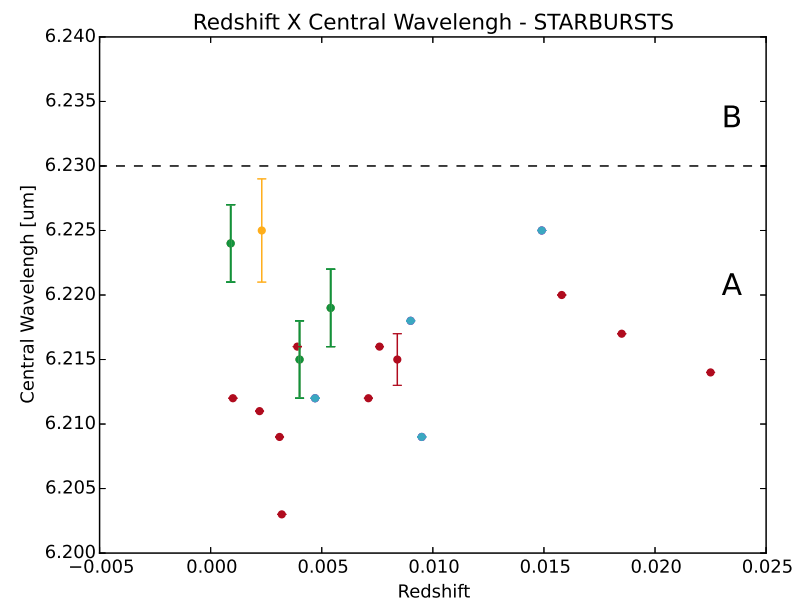

Figura 3.36: Recorte da Figura 3.35 com valores de redshift $<0.025$.

Dos três tipos mais abundantes, starbursts - e seus complementares regiões HII, Seyferts e LINER - dividem-se em $86.7 \%$ da classe A e 13.3\% da classe B. Quanto às Seyferts, $72.5 \%$ são da classe A e $27.5 \%$ são da B. Por último, ULIRGs possuem $75.7 \%$ da classe A, $20.3 \%$ da B e apenas $4 \%$ da classe C. Starbursts parecem ser os mais afetados por PANHs, mas as também altas porcentagens da classe A em ULIRGs e Seyferts podem indicar a forte influência destas moléculas nestes objetos. Se a contribuição de starbursts na emissão das 
outras galáxias é a causa destas elevadas estatísticas ou se isto se deve à ubiquidade dos PANHs, não se pode afirmar. Contudo, está bem claro a predominância da classe A na banda de $6.2 \mu m$ nestes diferentes tipos de galáxias.

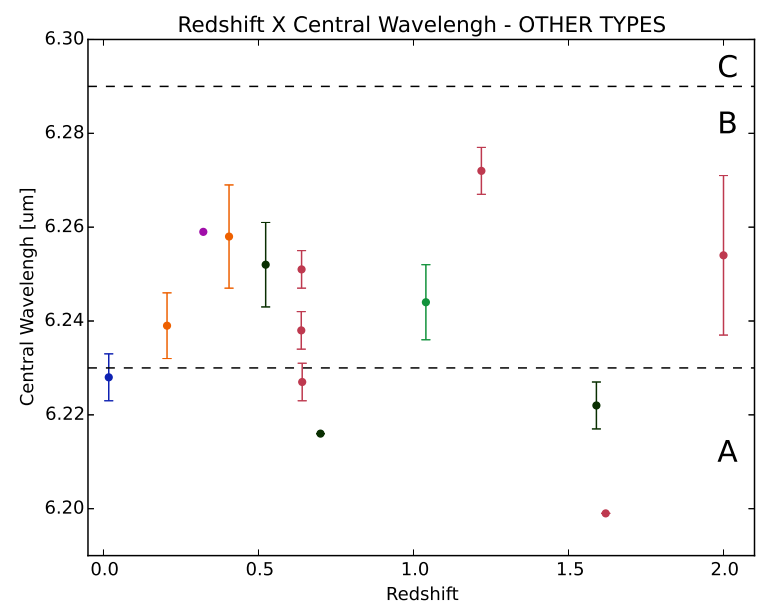

Figura 3.37: Gráfico do comprimento de onda central da banda de $6.2 \mu \mathrm{m}$ pelo redshift de outros tipos de galáxias. SMG - cor rosa; AGN2 - cor verde claro; FR-1 - cor azul; LINER - cor roxa; IRgal - cor verde escuro; e QSO - cor laranja.

\subsubsection{Espectros peculiares}

A distinção entre o melhor método de ajuste não é clara para alguns casos (Figuras 3.38 a 3.44). Para o primeiro objeto, 3C293 (Figuras 3.38 e 3.39), a posição do pico encontrada foi a mesma para ambos os ajustes e revelou pertencer a classe B. Houveram, entre eles, uma variação no RMS de 0.018 e outra de 0.001 no $\chi^{2}$ reduzido. Como, provavelmente, a cauda vermelha é melhor ajustada quando dois perfis são levados em conta, não há nada que indique a real existência de uma segunda banda para esta fonte.

Sobre o IRAS_00091-0738, os dois ajustes foram refeitos com novos intervalos de comprimento de onda e de comprimento de onda central $\left(\lambda_{i}\right)$, especificados diretamente na legenda dos respectivos gráficos, para adaptá-los ao fim da primeira banda. Enquanto o RMS com o minimize é o menor, o $\chi^{2}$ reduzido é menor com o curve_fit, levando a um impasse. Também não parece haver outra banda nesta região, uma vez que a ascensão vermelha ajustada com o minimize pode ser do contínuo do espectro, que se eleva até meados de 7.5 $\mu m$ (Figura B.15). Pelo primeiro (Figura 3.40), este objeto é um exemplar da classe B. Pelo segundo (3.41), da classe A. De qualquer forma, ambos mostram a presença de PANHs. 


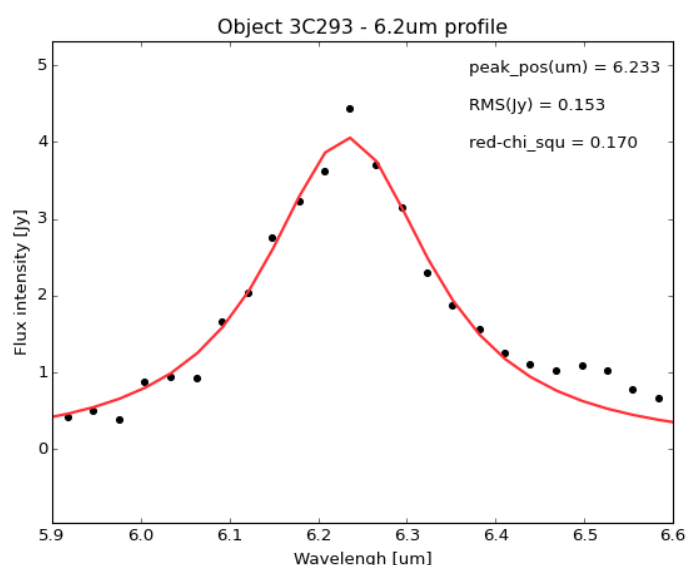

Figura 3.38: Perfil da banda de $6.2 \mu m$ do objeto 3C293 ajustado com curve_fit.

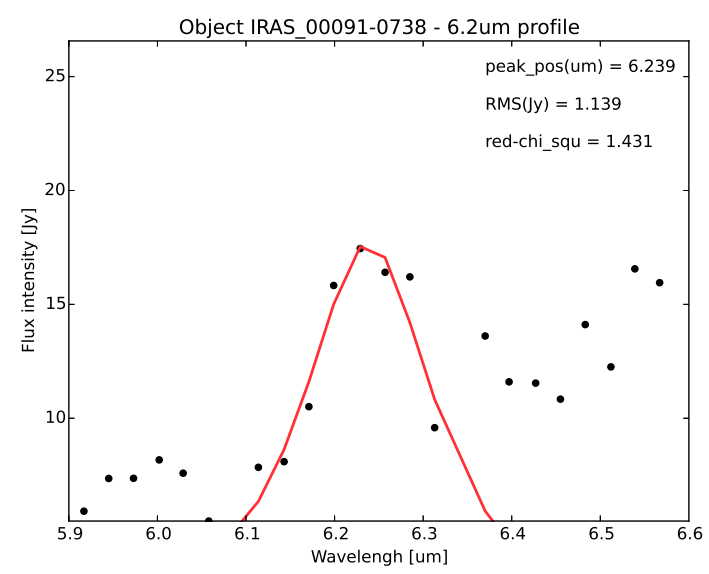

Figura 3.40: Perfil da banda de $6.2 \mu m$ do objeto IRAS_00091-0738 ajustado com curve_fit e para $6.10 \leq \lambda \leq 6.35$.

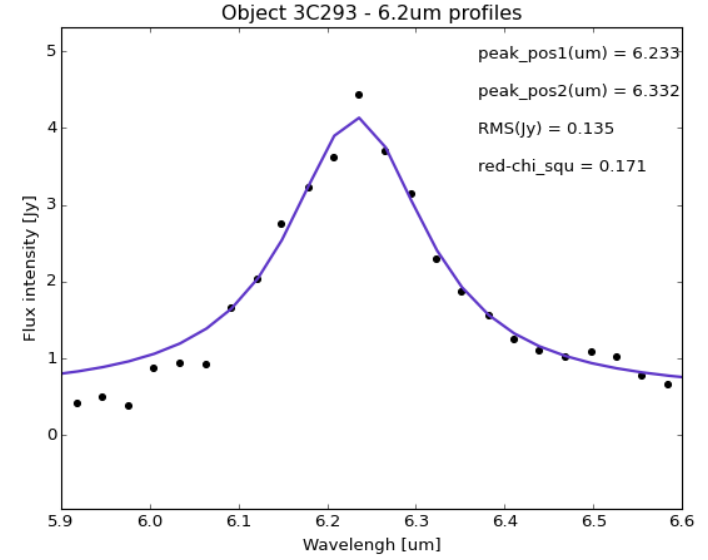

Figura 3.39: Perfil da banda de $6.2 \mu m$ do objeto 3C293 ajustado com minimize.

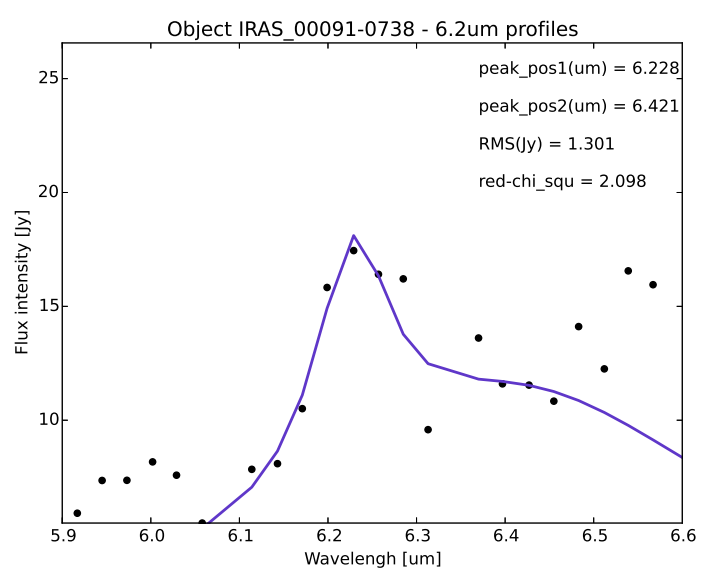

Figura 3.41: Perfil da banda de $6.2 \mu m$ do objeto IRAS_00091-0738 ajustado com minimize e para $6.10 \leq \lambda \leq 6.46,6.10 \leq \lambda_{i 1} \leq 6.27$ e $6.28 \leq \lambda_{i} 2 \leq$ 6.45 .

Em relação ao IRAS_11387+4116, as duas classificações foram classe B. Aparentemente, a adição da segunda banda torna o ajuste da primeira mais fiel aos dados. Contudo, a diferença entre os comprimentos centrais da banda de $6.2 \mu \mathrm{m}$, ajustados pelos dois programas, é pequena (0.007). Além disso, o patamar de 6.38 e 6.52 é razoavelmente bem descrito, considerando-se que se está usando um perfil de Drude para isto (Figura 3.43). Tanto o RMS como o $\chi^{2}$ reduzido são maiores para o ajuste único (Figura 3.42). Esta melhora no perfil ocasionada pela outra característica é mais vista para IRAS_15225+2350, cuja posição central do pico está claramente desviada para o vermelho na primeira situação (Figura 3.44). Mesmo que a segunda banda não seja muito confiável, não descrevendo esta 
espécie de característica de absorção por volta de $6.42 \mu m$, ela possibilita um retrato do perfil assimétrico de $6.2 \mu m$ com mais perfeição. Em especial, de sua ascensão azul.

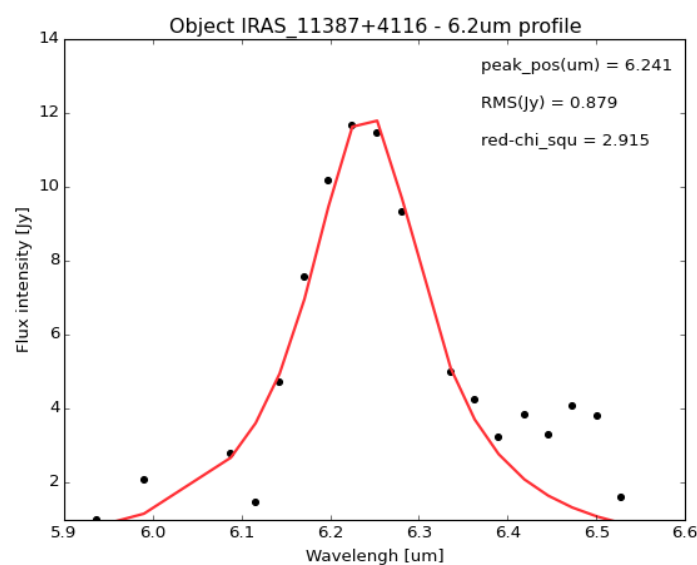

Figura 3.42: Perfil da banda de $6.2 \mu m$ do objeto IRAS_11387+4116 ajustado com curve_fit.

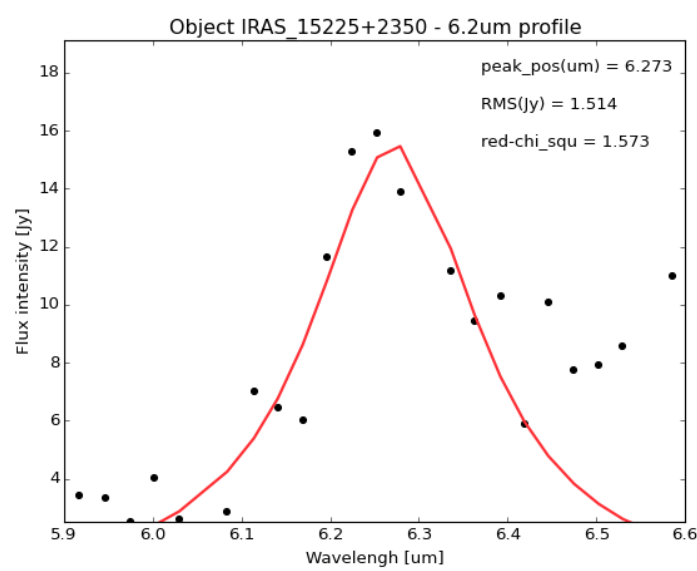

Figura 3.44: Perfil da banda de $6.2 \mu \mathrm{m}$ do objeto IRAS_15225+2350 ajustado com curve_fit.

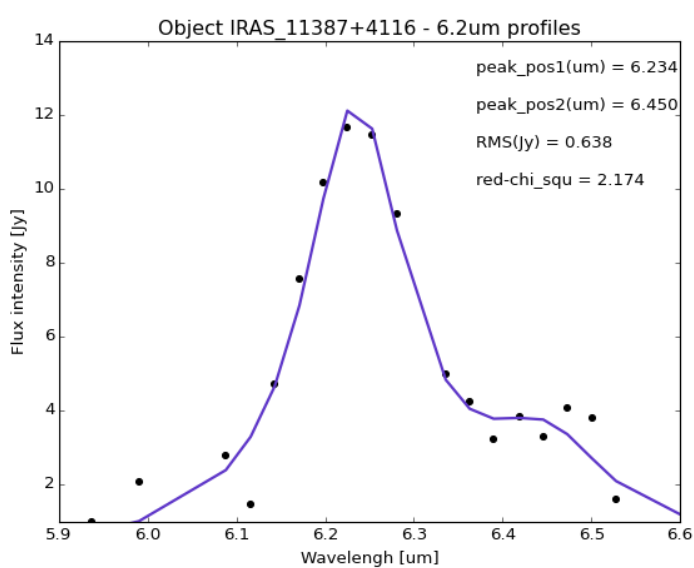

Figura 3.43: Perfil da banda de $6.2 \mu m$ do objeto IRAS_11387+4116 ajustado com minimize.

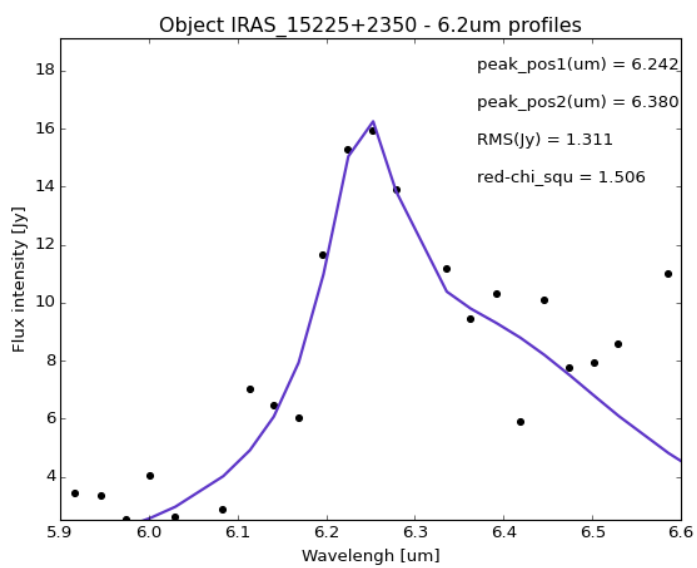

Figura 3.45: Perfil da banda de $6.2 \mu \mathrm{m}$ do objeto IRAS_15225+2350 ajustado com minimize.

Já o objeto MIPS16144 não parece nem um espectro de starburst. As duas bandas são extremamente evidentes, estando até ligeiramente espaçadas. Graças a esta distância, não foi possível ajustar ambas através do pah62_opt2.py. Esta questão foi abordada de forma diferente: empregou-se o pah62_curvefit.py duas vezes, uma para cada banda, e adequando-se os intervalos. 


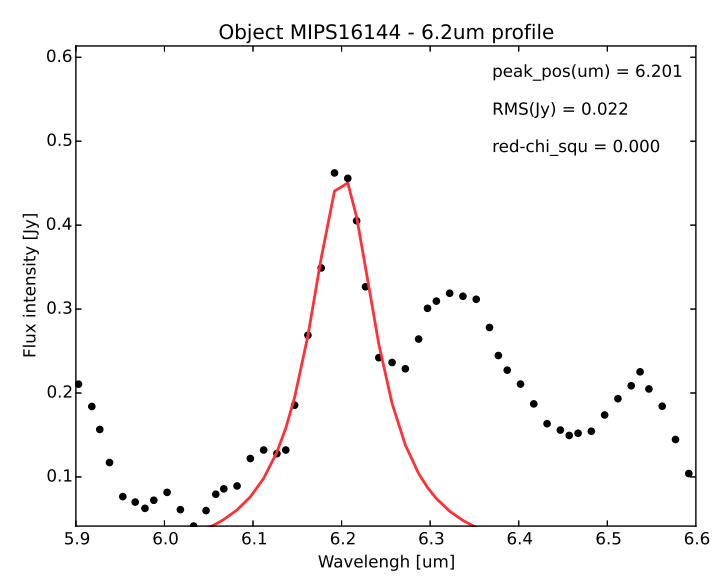

Figura 3.46: Perfil da banda de $6.2 \mu m$ do objeto MIPS16144 ajustado com curve_fit e para $6.1 \leq \lambda \leq 6.27$.

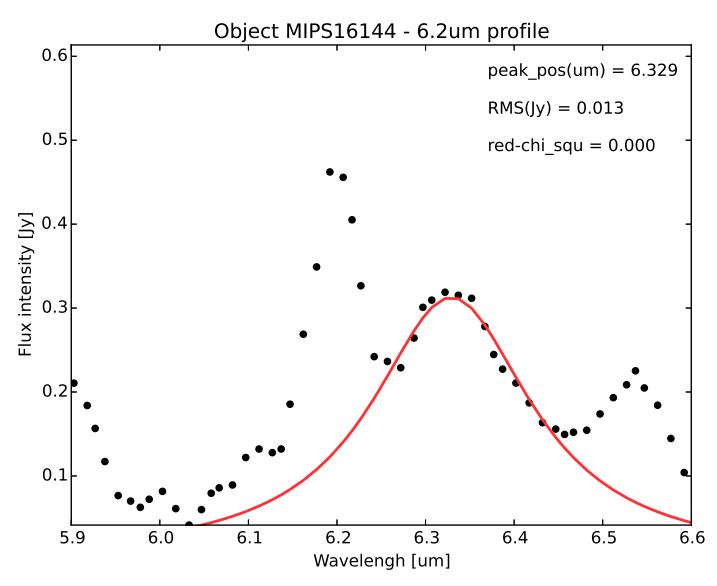

Figura 3.47: Perfil da banda de $6.3 \mu m$ do objeto MIPS16144 ajustado com curve_fit e para $6.26 \leq$ $\lambda \leq 6.47$ e $\lambda_{i}=6.35 \mu \mathrm{m}$.

Para a galáxia NGC7603 (Fig. 3.48), os intervalos do ajuste precisaram ser alterados por causa de uma aparente divisão do perfil em duas características, que não conseguiram ser descritas com o auxílio do minimize. Na verdade, parece haver uma absorção no perfil de emissão. Este objeto é bem brilhante (quando comparado com as fontes mais fracas analisadas aqui) e relativamente próximo, com um redshift de 0.03. Logo, não se trataria de um efeito devido a um fluxo débil, mas talvez de uma extração inadequada da fonte.

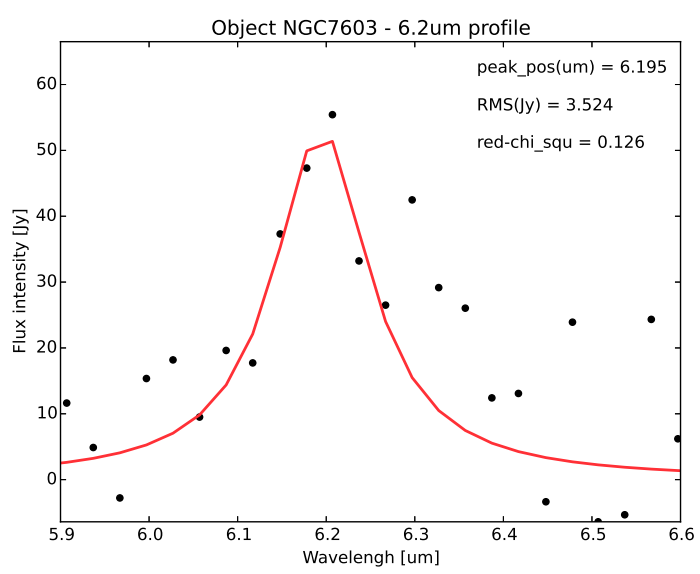

Figura 3.48: Perfil da banda de $6.2 \mu m$ do objeto NGC7603 ajustado com curve_fit e para $6.05 \leq \lambda \leq 6.28$.

Por fim, as últimas duas ocorrências que serão discutidas - MIPS506 e MIPS22699. Apesar dos valores baixos de RMS e $\chi^{2}$, o ajuste não representa satisfatoriamente as bandas. Pelo formato das emissões, eles apresentam o bump típico da classe C, que vem sendo relacionado com VSGs. 


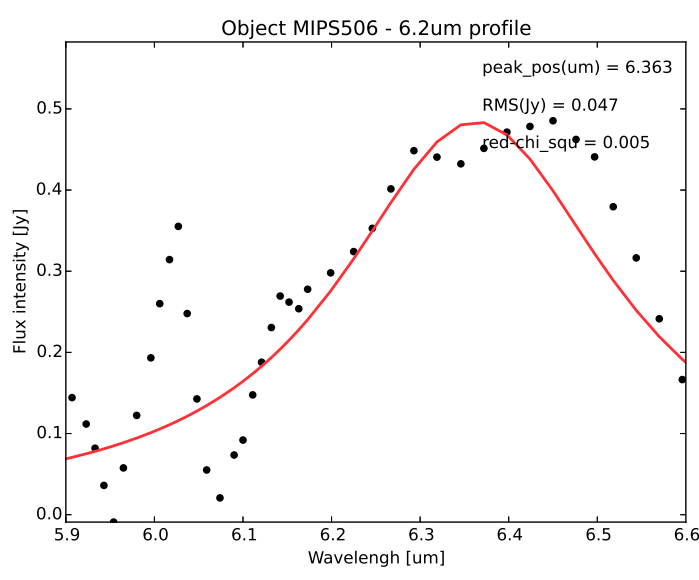

Figura 3.49: Perfil da banda de $6.2 \mu m$ do objeto MIPS506 ajustado com curve_fit.

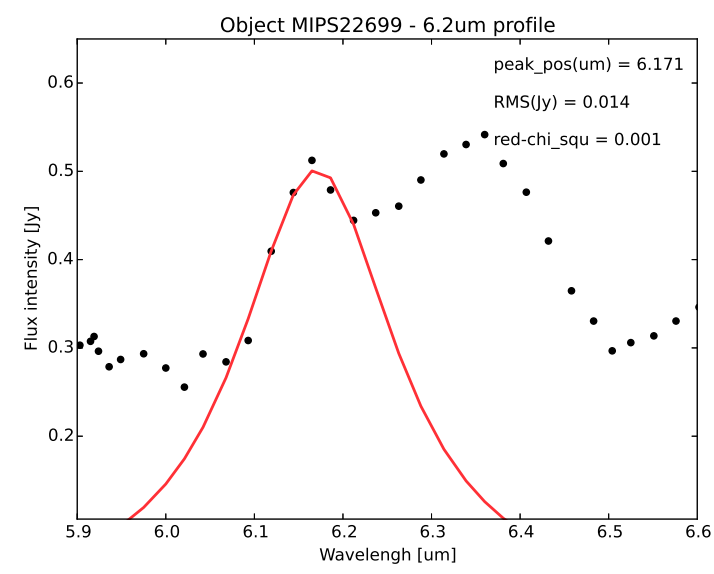

Figura 3.50: Perfil da banda de $6.2 \mu \mathrm{m}$ do objeto MIPS22699 ajustado com curve_fit e para $6.05 \leq$ $\lambda \leq 6.22$.

\subsubsection{Ajustes inconclusivos}

Para parte destes objetos, foi encontrada uma grande dificuldade de se distinguir qual a técnica mais adequada para se fazer o ajuste. Outros, nem conseguiram ser ajustados por ambas e somente uma é mostrada. Mesmo visualmente, não é simples de se enxergar a banda com exatidão por causa da alta variabilidade entre os pontos (Figuras 3.51 e 3.52). Para o NGC3031 (Figura 3.58), a banda se parece mais com um bump característico da classe C, como a própria posição central obtida comprova.

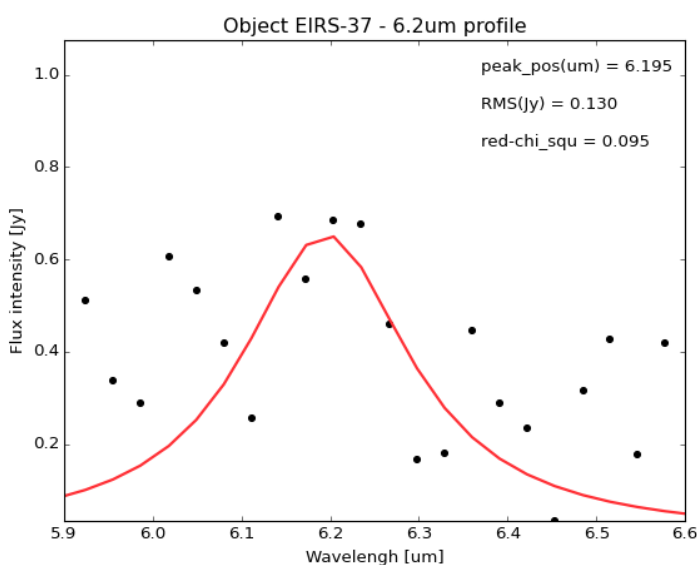

Figura 3.51: Perfil da banda de $6.2 \mu \mathrm{m}$ do objeto EIRS-37 ajustado com curve_fit.

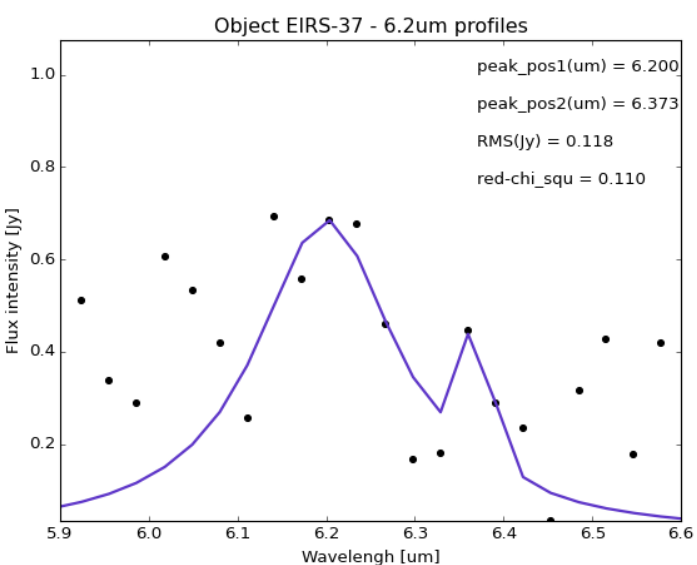

Figura 3.52: Perfil da banda de $6.3 \mu m$ do objeto EIRS-37 ajustado com minimize.

Em outras situações, a insegurança dos resultados pode ser explicada pelo fato de que muitos não contém uma quantidade de pontos suficiente para que o ajuste seja, pelo 
menos, confiável (Figuras 3.53 e 3.54). Portanto, não se pode analisar apropriadamente estas fontes por causa da falta de dados, implicando na necessidade de futuras observações com maior grau de resolução.

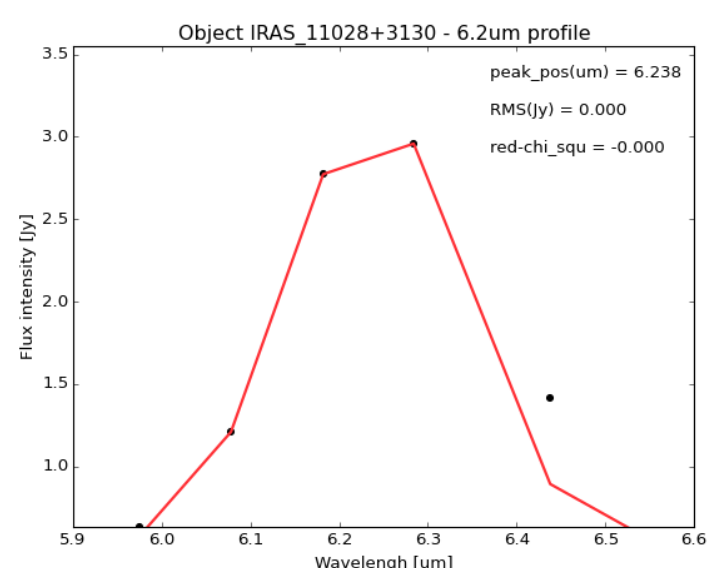

Figura 3.53: Perfil da banda de $6.2 \mu m$ do objeto IRAS_11028+3130 ajustado com curve_fit.

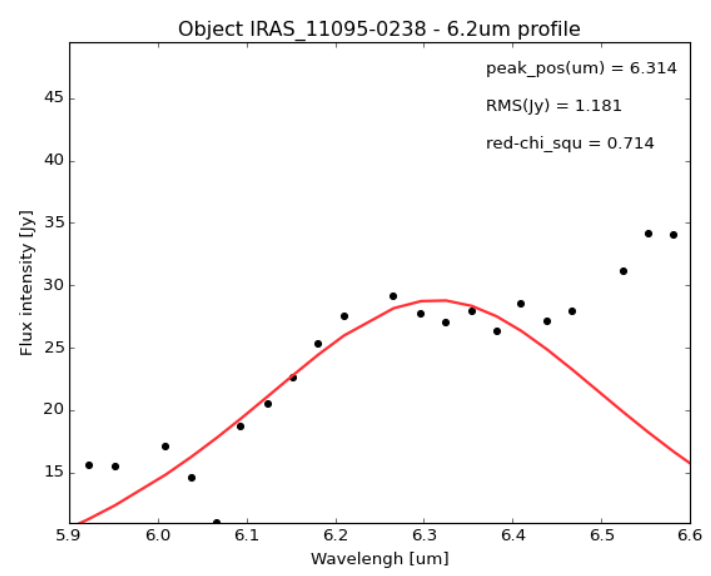

Figura 3.55: Perfil da banda de $6.2 \mu m$ do objeto IRAS_11095-0238 ajustado com curve_fit.

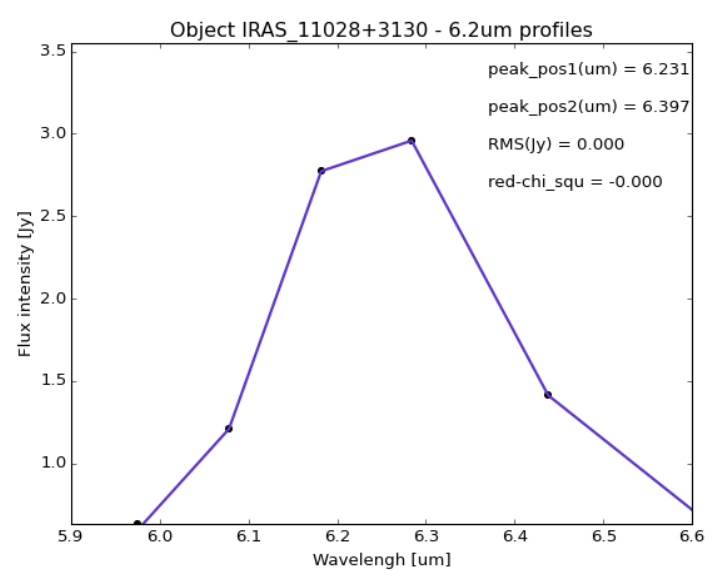

Figura 3.54: Perfil da banda de $6.3 \mu m$ do objeto IRAS_11028+3130 ajustado com minimize.

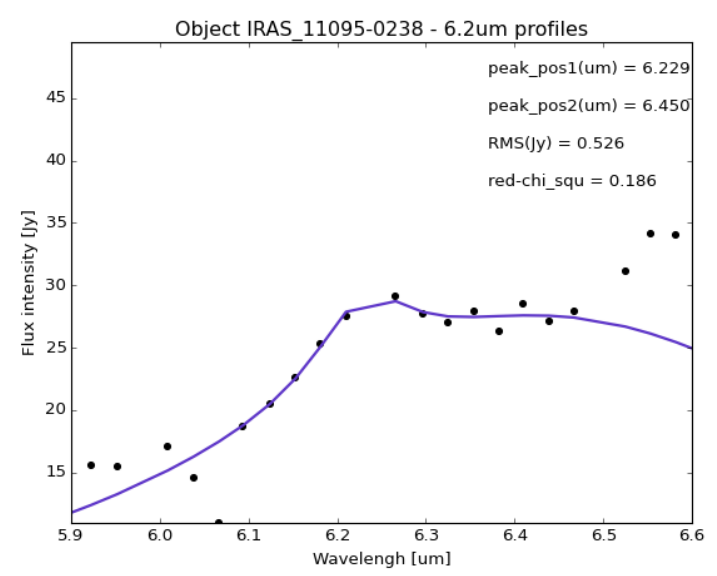

Figura 3.56: Perfil da banda de $6.3 \mu m$ do objeto IRAS_11095-0238 ajustado com minimize.

Para outros espectros, não parece haver a emissão em $6.2 \mu m$, como para o objeto IRAS_11095-0238 (Figuras 3.55 e 3.56), que se assemelha mais à plateaus. Há, ainda, aquelas fontes cujos espectros não recordam os de starbursts, como MIPS16059 (Figura 3.57) e SJ103809.18+583226.2 (Figura 3.59). O objeto SJ103809.18+583226.2 apresenta uma elevação do fluxo do lado azul da banda que, à primeira vista, é real e não um artifício do tratamento do espectro. E, MIPS16059 exibe uma estrutura com um bump ao vermelho e, do lado azul, uma característica de emissão e duas de absorção. Neste último caso, em 
particular, a qualidade do perfil é muito alta. Em ambos os casos, portanto, é provável que se tratem de características reais, cujas origens ainda devem ser elucidadas.

Os demais objetos podem ser vistos no Apêndice C.3.

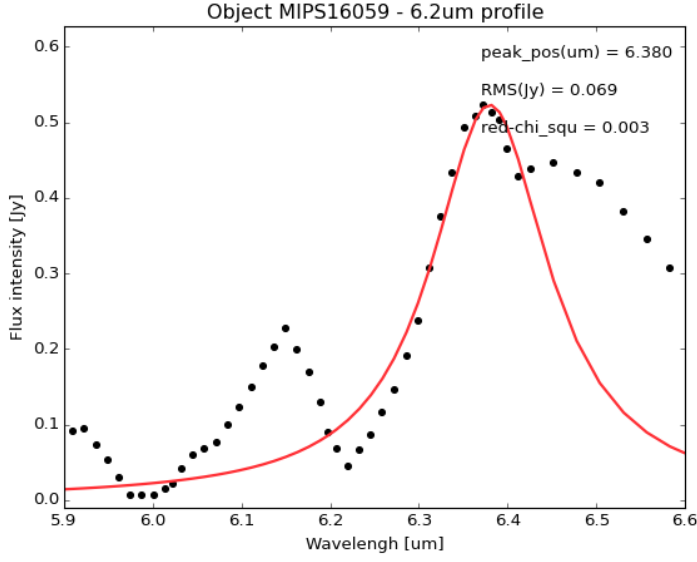

Figura 3.57: Perfil da banda de $6.2 \mu \mathrm{m}$ do objeto MIPS16059 ajustado com curve_fit.

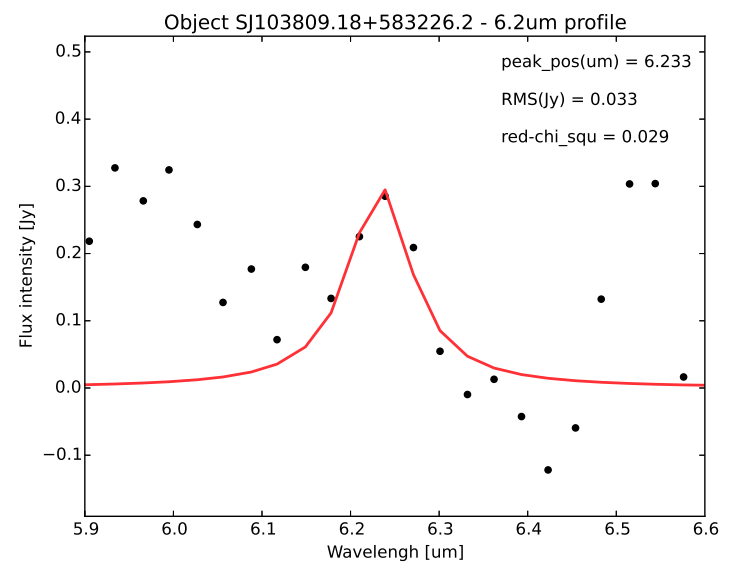

Figura 3.59: Perfil da banda de $6.2 \mu \mathrm{m}$ do objeto SJ103809.18+583226.2 ajustado com curve_fit e para $6.15 \leq \lambda \leq 6.35$.

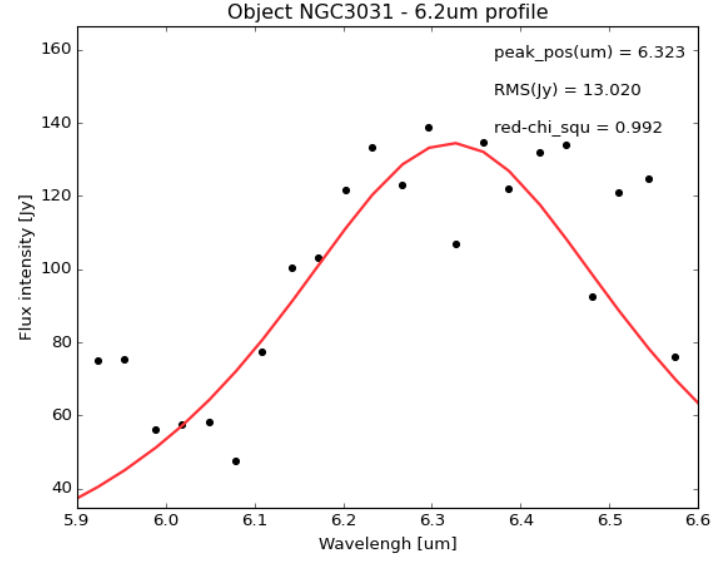

Figura 3.58: Perfil da banda de $6.3 \mu m$ do objeto NGC3031 ajustado com curve_fit.

\subsection{A banda de $6.0 \mu m$}

Nesta região do IR médio que está sendo estudada aqui, espectros de laboratório de gelo d'água já destacaram características em $3.1 \mu m$ do modo de estiramento do $O-H$, em 4.5 $\mu \mathrm{m}$ do modo de combinação, em $6.0 \mu m$ do modo de deformação angular do $O-H$ e em $12 \mu m$ do modo libracional (Robinson et al., 2012). No ISM, pequenos grãos de gelo, 
menores que $0.3 \mu m$, estão ligados à regularização da transferência de energia radiativa dentro das nuvens, determinando seus respectivos balanço de energia e espectro, além de influenciar na sua evolução e na formação estelar (Hudgins et al., 1993).

Todas as bandas citadas são vistas em absorção por causa da baixa temperatura de sublimação do gelo d'água em ambientes interestelares $(T \lesssim 150 K)$, podendo variar na forma e no comprimento de onda central se o gelo for cristalino, amorfo ou conter impurezas (Robinson et al., 2012). Os espectros infravermelhos de grãos de poeira de nuvens moleculares densas são dominados por elas, cujas produções envolvem, normalmente, a superposição de efeitos de absorção e reflexão de gelos e suas superfícies (Hudgins et al. 1993).

As primeiras detecções de características de gelo em objetos extragalácticos se deram nas galáxias NGC4418 e a IRAS00183-7111, uma ULIRG distante (Spoon et al., 2001). E, segundo Spoon et al. (2002), estas características se encontram na maioria das ULIRGs, que possuem grandes quantidades de material molecular, mas são fracas ou ausentes em diversas galáxias Seyferts e starbursts.

Em especial, a banda de $6.0 \mu m$ é atribuída ao gelo d'água amorfo e é bastante afetada por efeitos reversíveis de expansão e encolhimento térmicos, causados por oscilações cíclicas de temperatura de $10 \mathrm{~K}$ a $120 \mathrm{~K}$, que modificam ligeiramente a largura da banda e a fazem discrepar de acordo com a fonte observada (Spoon et al., 2002).

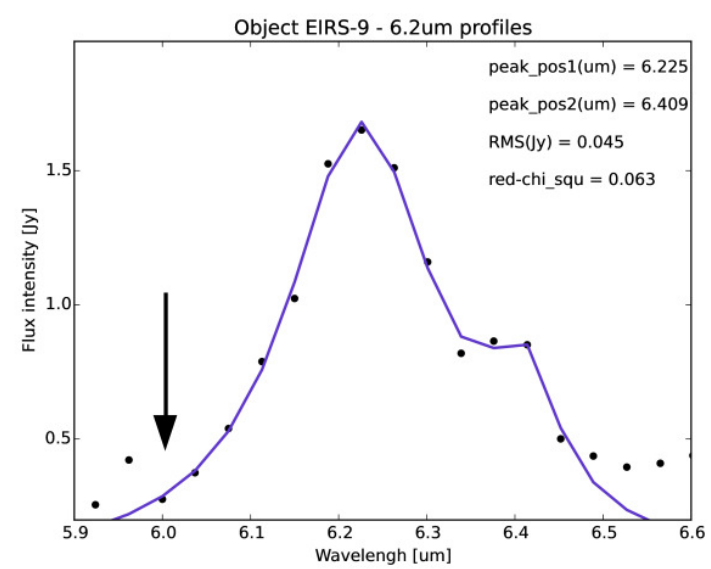

Figura 3.60: Banda de 6.0 $\mu \mathrm{m}$ indicada pela seta preta presente no objeto EIRS-9.

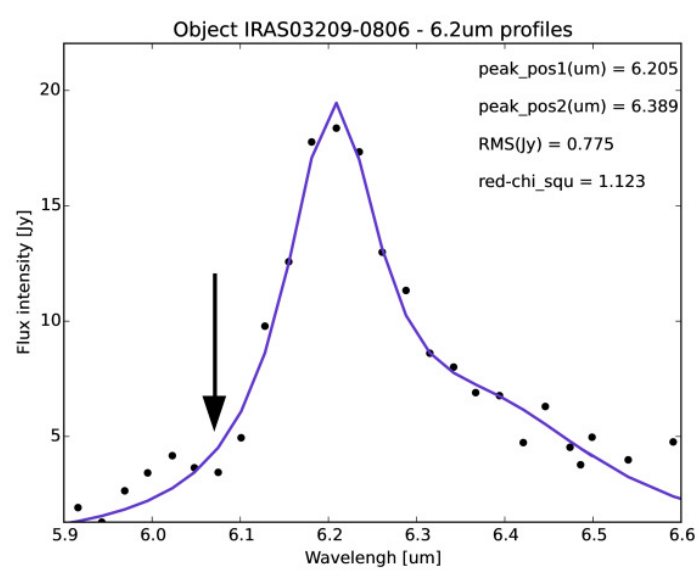

Figura 3.61: Banda de 6.0 $\mu \mathrm{m}$ indicada pela seta preta presente no objeto IRAS03209-0806.

Embora esta banda não tenha sido ajustada e analisada com mais detalhes neste trabalho, algumas galáxias em que ela estava presente estão destacadas logo a seguir - a banda 
está indicada por uma seta preta. Normalmente, espera-se que poucas galáxias apresentem um perfil de absorção perto de $6.0 \mu m$ (Figura 3.60), o que é uma consequência do fato de que emissões muito intensas de PAH evitam a detecção destas características quando elas são só moderadamente fortes (Spoon et al., 2002). Contudo, um comportamento imprevisto foi notado. Para alguns objetos, a banda aparece deslocada para o vermelho, com uma posição de pico por volta de $6.07 \mu m$, como mostrado nas Figuras 3.61 , 3.62 e 3.63 .

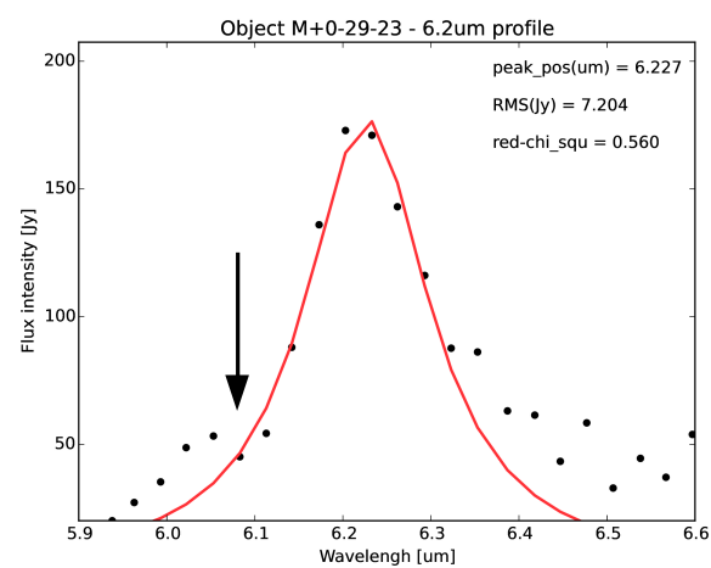

Figura 3.62: Banda de 6.0 $\mu \mathrm{m}$ indicada pela seta preta presente no objeto $\mathrm{M}+0-29-23$.

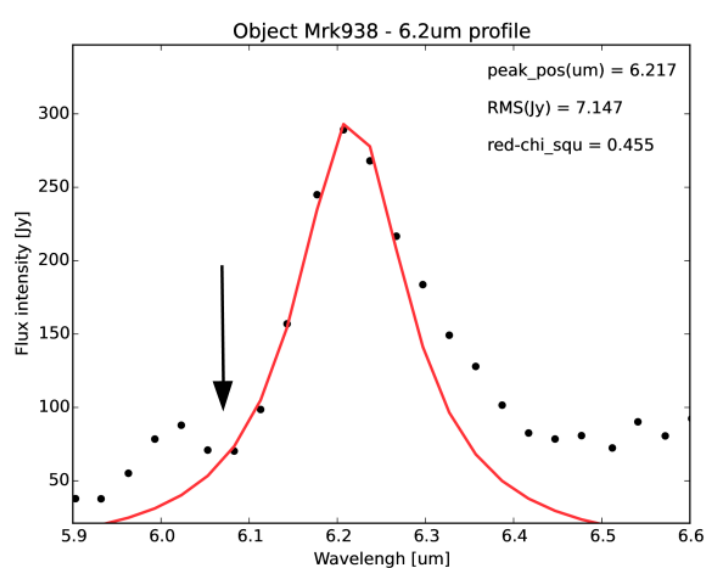

Figura 3.63: Banda de $6.0 \mu \mathrm{m}$ indicada pela seta preta presente no objeto Mrk938. 
Capítulo 4

\section{Conclusões e perspectivas}

Das 169 galáxias com emissão dominada por starbursts bem ajustadas, $60 \%$ (124) foram classificadas como classe A, 20\% (42) como classe B e 1.5\% (3) como classe C. A classe A, correspondente a um comprimento de onda central perto de $6.22 \mu m$, só foi explicada pela substituição de carbono por nitrogênio, apesar de outras tentativas de explanação (Hudgins et al., 2005). Além disso, a classe B pode representar uma mistura entre PAHs e PANHs. Logo, PANHs parecem dominar estes espectros, o que pode indicar outro reservatório de nitrogênio no Universo, com condições de densidade e temperatura diferentes das fases gasosas e gelos. O fato de somente algumas fontes pertencerem à classe $\mathrm{C}$ pode significar que a maioria dos PAHs já incorporaram nitrogênio nos seus anéis.

De acordo com estes resultados, além dos PAHs representarem uma poderosa fonte de luminosidade no infravermelho, os PANHs estariam presentes em quantidades significativas no meio interestelar de galáxias. A ubiquidade dos PANHs fornece um apoio à proposta, dentro do cenário do Mundo Aromático para a origem da vida, de que eles teriam um papel relevante no surgimento da vida na Terra e em outras partes do Universo. Os PANHs não apenas estão presentes no ISM de starbursts, mas também são responsáveis por uma fração importante da emissão no IR, especialmente para a banda de $6.2 \mu \mathrm{m}$.

Eles também podem influenciar as bandas de 3.3, 7.7 e 11.2 $\mu m$ (Van Diedenhoven et al. 2004), que estão precisando de mais estudos para estas galáxias. Um próximo passo seria analisar estas características para procurar uma conexão entre as classes de Peeters para um mesmo objeto e para confirmar os resultados obtidos aqui.

Quantos aos objetos que não foram considerados para a estimativa final (Seções 3.2.1.4 e 3.2.1.5, somente alguns fazem parte da classe C. Embora não se possa afirmar qual a classe (A ou B) dos restantes, todos contêm PANHs e dão mais suporte para as conclusões 
discutidas acima. Para um grau de confiabilidade maior, estas galáxias necessitam de novas observações com maior resolução ou outros métodos de análise que comportem suas peculiaridades.

\subsection{Novas abordagens}

Como já foi dito no Capítulo 2, a análise da banda de $6.2 \mu m$ depende da estimativa do redshift das fontes para a correção do espectro e, para alguns casos, este valor ou não é preciso o suficiente, ou foi extraído das próprias bandas de PAHs. Para solucionar esta questão, foi utilizada uma abordagem estatística neste trabalho. Todavia, uma outra possibilidade também fora avaliada.

Uma vez que as bandas de 6.2 e $7.7 \mu m$ estão relacionadas (Seção 1.4.1), a segunda poderia indicar a presença de PANHs mesmo que a primeira não tenha sido observada. Além disso, Van Diedenhoven et al. (2004) constatou que, em alguns casos, as classes estão correlacionadas ao longo do espectro, principalmente a A. Ou seja, se as bandas de $6-9 \mu m$ são da classe A, a de $11.2 \mu m$ (e de $3.3 \mu m$ ) também será. E, embora esta conexão dependa do tipo do objeto, ambientes protoestelares/protoplanetários ou com formação de poeira parecem ser fundamentais para mudanças nos perfis de 3.3 ou $11.2 \mu m$. Com isso, é possível extrair as informações desejadas calculando-se as distâncias entre as bandas, pelo menos para starbursts, como esquematizado na Figura 4.1.

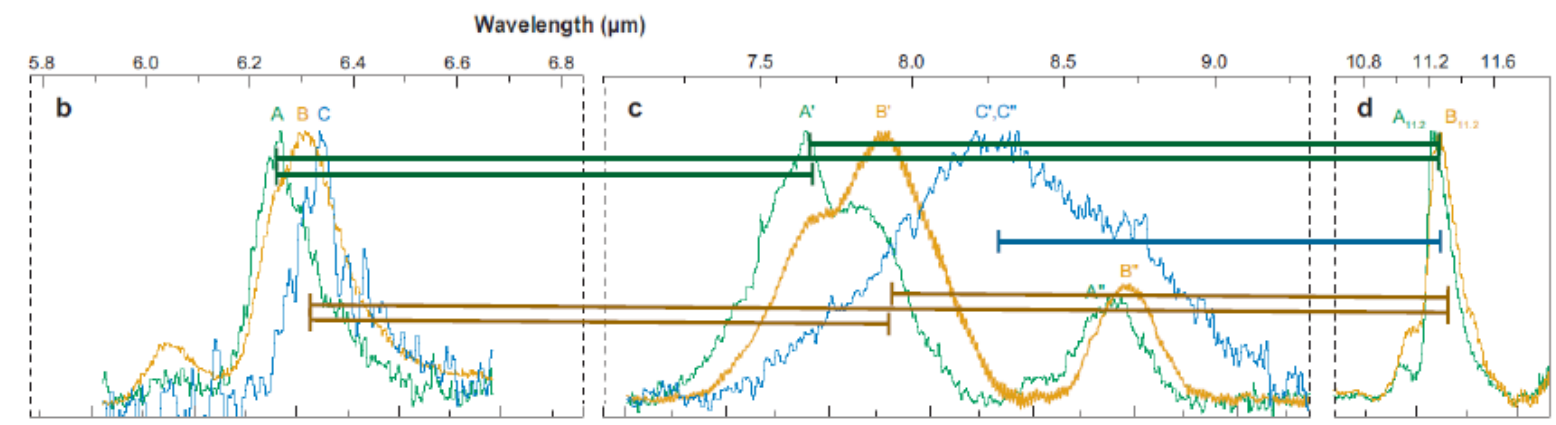

Figura 4.1: Imagem representativa dos espectros das classes de Peeters, retirada de Tielens (2008), mostrando as distâncias entre cada banda que podem ser calculadas. Dessa forma, pode-se classificar as galáxias com mais segurança e independentemente do redshift.

Esta técnica é interessante por três razões principais: a) ela é independente do redshift, o que solucionaria a questão das galáxias que tiverem seus redshifts derivados das bandas de PAHs; b) a banda de $7.7 \mu m$ apresenta variações nas posições dos picos mais espaçadas 
do que as demais emissões e, portanto, permite uma análise menos atrelada à resolução das fontes; e c) ao se utilizar mais de uma banda, pequenas variações nas características de cada perfil relacionadas com a fonte influenciam menos no resultado final e a classificação da galáxia depende menos de suas singularidades.

As figuras abaixo (4.2 e 4.3) exibem um teste realizado para ajustar as bandas de PAHs da seguinte maneira: banda de $6.2 \mu m$ - Lorentziana, banda de $7.7 \mu m$ - perfis de Drude e banda de 11.2 $\mu m$ - Gaussiana. Os palpites iniciais (Tabela 4.1) também foram extraídos de Smith et al. (2007). Tentou-se adequar o melhor possível o modelo com a estrutura das bandas e, por isso, diferentes perfis foram escolhidos. Foi percebida uma dificuldade em analisar e ajustar todos simultaneamente, em especial a banda de $7.7 \mu m$, que varia bastante de acordo com a classe de Peeters a qual o objeto pertence. Assim, pode ser mais aconselhável estudar cada uma separadamente, como feito para a banda de $6.2 \mu \mathrm{m}$ e, posteriormente, agregar os resultados. Por os ajustes ainda precisarem ser melhorados, as distâncias entre as bandas não foram calculadas. Porém, considerando-se somente a forma das emissões e, principalmente, da banda em 7.7 $\mu$ m (Seção 1.4.1), ambas as galáxias parecem pertencer a classe A. Segundo os resultados deste trabalho, o objeto 3C31 realmente é da classe A e o 3C293 é da B.

Tabela 4.1 - Palpites iniciais para o ajuste das bandas de PAHs.

\begin{tabular}{lcc}
\hline \hline Banda / cor do ajuste & $\lambda_{i}(\mu \mathrm{m})$ & $\gamma_{i}$ \\
\hline $6.2 \mu \mathrm{m} / \mathrm{azul}$ & 6.22 & 0.187 \\
& 6.69 & 0.070 \\
\hline \multirow{2}{*}{$7.7 / 8.6 \mu \mathrm{m} /$ vermelho } & 7.42 & 0.126 \\
& 7.60 & 0.044 \\
& 7.85 & 0.053 \\
& 8.33 & 0.050 \\
& 8.61 & 0.039 \\
\hline $10.6 \mu \mathrm{m} /$ verde & 10.68 & 0.020 \\
\hline $11.2 \mu \mathrm{m} /$ magenta & 11.23 & 0.012 \\
& 11.33 & 0.032 \\
\hline
\end{tabular}




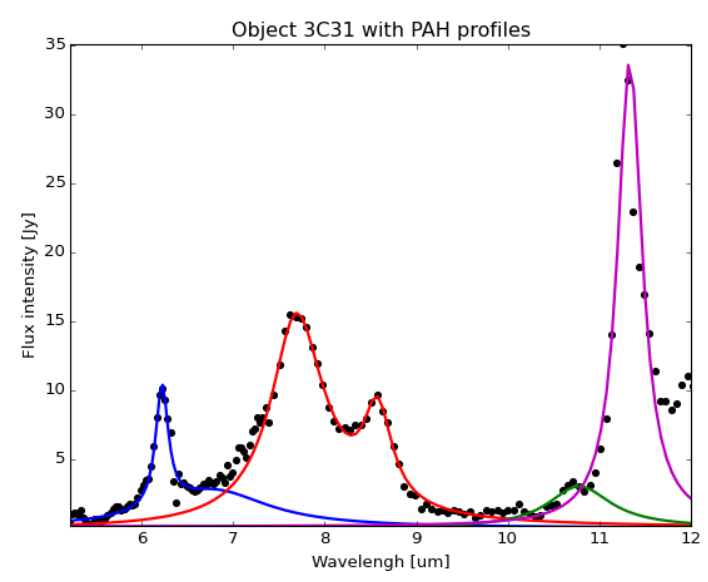

Figura 4.2: bandas de PAHs do objeto 3C31.

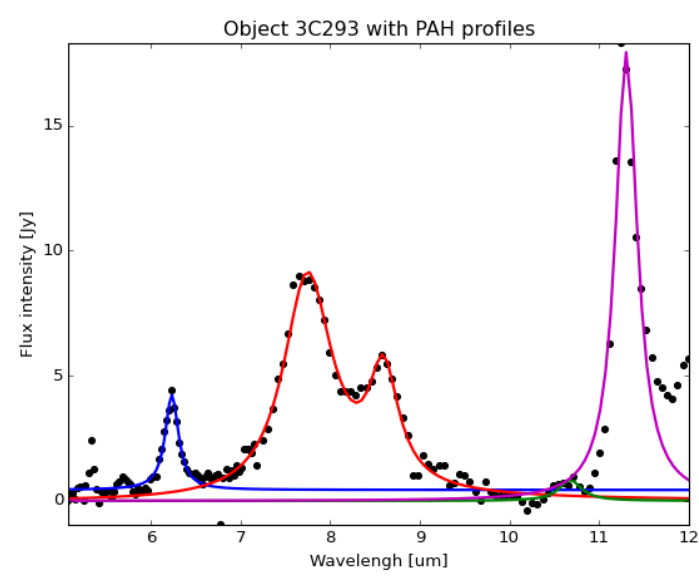

Figura 4.3: bandas de PAHs do objeto 3C293.

Outro programa que pode ajudar a entender as características das emissões de PAHs é o PAHTAT (sigla de PAH Toulouse Astronomical Templates). Criado por Pilleri et al. (2012), é uma ferramenta em IDL que interpreta espectros com estas emissões usando quatro modelos - PAH neutro $\left(P A H^{0}\right)$, cátion $\mathrm{PAH}\left(P A H^{+}\right)$, grandes $\mathrm{PAH}$ ionizados $\left(P A H^{x}\right)$ e eVSGs (VSG em evaporação). Ele ainda ajusta o contínuo e algumas bandas moleculares. Foi realizado um teste, sem modificar o programa original, para a galáxia NGC4818 (Figura 4.4). Entretanto, será preciso adicionar outros componentes para se estudar os objetos astrofísicos diretamente com ele, tais como outras emissões iônicas e moleculares (já abordadas pelo PAHFIT). Ou, como feito nesta dissertação, estas contribuições podem ser cobertas primeiramente com o PAHFIT e subtraídas, para depois os espectros passarem pelo PAHTAT. 


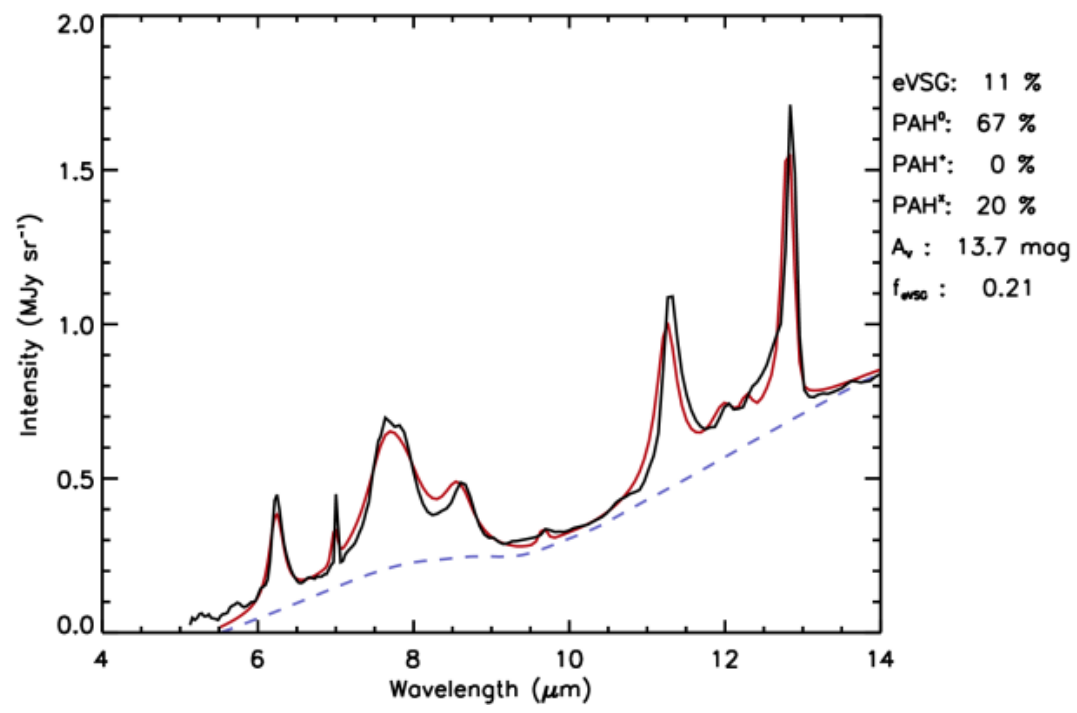

Figura 4.4: Espectro do objeto NGC4818 ajustado com o PAHTAT. As siglas $A_{v}$ e $f_{\text {evsg }}$ são, respectivamente, a magnitude da extinção visual ao longo da linha de visada e a taxa da emissão total de eVSGs pela emissão total AIB (traçadora da evolução dos eVSGs em diferentes ambientes).

\subsection{Outros ambientes astrofísicos e novas observações}

Outros objetos extragalácticos também apresentam bandas de PAH, como AGNs, por exemplo. Com o auxílio do projeto Spitzer/IRS ATLAS, amostras destas galáxias podem ser estudadas pelo mesmo processo realizado neste trabalho. Com isso, o conhecimento sobre PANHs e as classes de Peeters pode abranger variados tipos de fontes. Ademais, objetos bem mais próximos e propícios para a existência de PAH e PANH são os cometas e asteroides do Sistema Solar. Estudos recentes mostraram a alta probabilidade de formação de nucleobases e aminoácidos nestes ambientes - através da irradiação da formamida $\left(\mathrm{NH}_{2} \mathrm{CHO}\right)$ por prótons (Saladino et al., 2015).

Já em relação às novas obervações, o James Webb Space Telescope (JWST) será o maior telescópio científico já lançado no espaço - 42 vezes maior que o Spitzer - e cobrirá de 0.6 a $28.6 \mu \mathrm{m}$, podendo detectar a banda de $6.2 \mu \mathrm{m}$ com maior resolução e até redshift de 3.6 (Bernard-Salas, 2015). Assim sendo, esta dissertação é uma prévia do que se esperar das futuras observações de starbursts para esta banda. Sob outra perspectiva, este telescópio poderá confirmar os resultados e proporcionar novos entendimentos sobre este assunto. Por exemplo, contemplando a banda de $3.3 \mu \mathrm{m}$, que também sofre interferência de PANHs (Hudgins et al., 2005). 


\section{Referências Bibliográficas}

Andrews H., Boersma C., Werner M. W., Livingston J., Allamandola L. J., Tielens A. G. G. M., PAH EMISSION AT THE BRIGHT LOCATIONS OF PDRs: THE grandPAH HYPOTHESIS, ApJ, 2015, vol. 807, p. 99

Bernard-Salas J., Spectroscopic studies of PAHs with the James Webb Space Telescope., AstroPAH, 2015, vol. 24, p. 4

Berné O., Montillaud J., Joblin C., Top-down formation of fullerenes in the interstellar medium., A\&A, 2015, vol. 577, p. A113

Brandl B. R., Bernard-Salas J., Spoon H. W. W., Devost D., Sloan G. C., Guilles S. ans Wu Y., et al. The Mid-Infrared Properties of Starburst Galaxies from Spitzer-IRS Spectroscopy., ApJ, 2006, vol. 653, p. 1129

Buchanan C. L., Gallimore J. F., O’Dea C. P., Baum S. A., Axon D. J., Robinson A., Elitzur M., Elvis M., Spitzer IRS Spectra of a Large Sample of Seyfert Galaxies: A Variety of Infrared Spectral Energy Distributions in the Local Active Galactic Nucleus Population., ApJ, 2006, vol. 132, p. 401

Dasyra K. M., Yan L., Helou G., Sajina A., Fadda D., Zamojski M., Armus L., Draine B., Frayer D., The 0.9 mJy Sample: A Mid-Infrared Spectroscopic Catalog of 150 Infrared-Luminous, $24 \mu \mathrm{m}$ Selected Galaxies at $0.3 \leq z \leq 3.5$., ApJ, 2009, vol. 701, p. 1123

Deo R. P., Crenshaw D. M., Kraemer S. B., Dietrich M., Elitzur M., Teplitz H., Turner T. J., Spitzer IRS Observations of Seyfert 1.8 and 1.9 Galaxies: A Comparison with Seyfert 1 and Seyfert 2., ApJ, 2007, vol. 671, p. 124 
Dwek E., Arendt R. G., Fixsen D. J., Sodtroski T. J., et al. Detection and Characterization of Cold Interstellar Dust and Polycyclic Aromatic Hydrocarbon Emission, from COBE Observations, ApJ, 1997, vol. 475, p. 565

Ehrenfreund P., Irvine W., Becker L., Blank J., Brucato J.and Colangeli L., Derenne S., Despois D., Dutrey A., Fraaije H., Lazcano A., Owen T., Robert F., Astrophysical and astrochemical insights into the origin of life., Reports on Progress in Physics, 2002, vol. 65 , p. 1427

Ehrenfreund P., Rasmussen S., Cleaves J., Chen L., Experimentally tracing the key steps in the origin of life: The aromatic world., Astrobiology, 2006, vol. 6, p. 490

Farrah D., Weedman D., Lonsdale C. J., Polletta M., Rowan-Robinson M., Houck J. ans Smith H. E., Mid-Infrared Spectroscopy of Optically Faint Extragalactic 70 $\mu m$ Sources., ApJ, 2009, vol. 696, p. 2044

Guimarães M. C., Efeitos da Presença de Poeira na Emissão de Galáxias a Altos e Baixos Redshifts, Universidade de São Paulo, 2006, Tese de Doutorado, 203 p.

Hernán-Caballero A., Hatziminaoglou E., An atlas of mid-infrared spectra of star-forming and active galaxies., MNRAS, 2011, vol. 414, p. 500

Hernán-Caballero A., Pérez-Fournon I., Hatziminaoglou E., Afonso-Luis A. ans RowanRobinson M., Rigopoulou D., Farrah D., et al. Mid-infrared spectroscopy of infraredluminous galaxies at $z \sim 0.5-3$. , MNRAS, 2009, vol. 395, p. 1695

Higdon S. J. U., Devost D., Higdon J. L., Brandl B. R., Houck J. R., Hall P., Barry D., Chamandaris V., Smith J. D. T., Sloan G. C., Green J., The SMART Data Analysis Package for the Infrared Spectrograph on the Spitzer Space Telescope., PASP, 2004, vol. 116 , p. 975

Hiner K. D., Canalizo G., Lacy M., Sajina A., Armus L., Ridgway S., Storrie-Lombardi L., An Infrared Comparison of Type-1 and Type-2 Quasars., ApJ, 2009, vol. 706, p. 508

Houck J. R., Roellig T. L., van Cleve J., Forrest W. J., Herter T., Lawrence C. R., Matthews K., Reitsema H. J., Soifer B. T., Watson D. M., Weedman D., Huisjen M., Troeltzsch J., Barry D. J., Bernard-Salas J., Blacken C. E., Brandl B. R., et al. The 
Infrared Spectrograph (IRS) on the Spitzer Space Telescope., The Astrophysical Journal Supplement Series, 2004, vol. 154, p. Issue 1, 18

Hudgins D. M., Allamandola L. J., The spacing of the interestellar 6.2 and 7.7 micron emission features as an indicator of polycyclic aromatic hydrocarbon size., ApJ, 1999, vol. 513, p. L69

Hudgins D. M., Bauschlicher J. C. W., Allamandola L. J., Variations in the peak position of the $6.2 \mu \mathrm{m}$ interstellar emission feature: a tracer of $\mathrm{N}$ in the interstellar polycyclic aromatic hydrocarbon population., ApJ, 2005, vol. 632, p. 316

Hudgins D. M., Sandoford A., Allamandola L. J., Tielens A. G. G. M., Mid- and far-infrared spectroscopy of ices: optical constants and integrated absorbances., ApJS, 1993, vol. 86, p. 713

Imanishi M., Luminous Buried Active Galactic Nuclei as a Function of Galaxy Infrared Luminosity Revealed through Spitzer Low-resolution Infrared Spectroscopy., ApJ, 2009, vol. 694 , p. 751

Imanishi M., Dudley C. C., Maiolino R., Maloney P. R., Nakagawa T., Risaliti G., A Spitzer IRS Low-Resolution Spectroscopic Search for Buried AGNs in Nearby Ultraluminous Infrared Galaxies: A Constraint on Geometry between Energy Sources and Dust., ApJ, 2007, vol. 171 , p. 72

Imanishi M., Maiolino R., Nakagawa T., Spitzer Infrared Low-Resolution Spectroscopic Study of Buried Active Galactic Nuclei in a Complete Sample of Nearby Ultraluminous Infrared Galaxies., ApJ, 2010, vol. 709, p. 801

Joblin C., Léger A., Martin P., Contribution of polycyclic aromatic hydrocarbon molecules to the interstellar extinction curve., ApJ, 1992, vol. 393, p. L79

Kemper F., Vriend W. J., Tielens A. G. G. M., The Absence of Crystalline Silicates in the Diffuse Interstellar Medium, ApJ, 2004, vol. 609, p. 826

Lebouteiller V., Bernard-Salas J., Sloan G. C., Barry D. J., Advanced Optimal Extraction for the Spitzer/IRS., PASP, 2010, vol. 122, p. 231 
Leipski C., Antonucci R., Ogle P., Whysong D., The Spitzer View of FR I Radio Galaxies: On the Origin of the Nuclear Mid-Infrared Continuum., ApJ, 2009, vol. 701, p. 981

Li A., Interaction of Nanoparticles with Radiation, ASP Conference Series, Astrophysics of Dust, 2004, vol. 309, p. 417

Li A., Draine B. T., Infrared Emission from Interstellar Dust. II. The Diffuse Interstellar Medium, Apj, 2001, vol. 554, p. 778

Materese C. K., Nuevo M., Sandford S. A., N- AND O -HETEROCYCLES PRODUCED FROM THE IRRADIATION OF BENZENE AND NAPHTHALENE IN H2O/NH3CONTAINING ICES, ApJ, 2015, vol. 800, p. 116

Menéndez-Delmestre K., Blain A. W., Smail I., Alexander D. M., Chapman S. C., Armus L., Frayer D., Ivison R. J., Teplitz H., Mid-Infrared Spectroscopy of Submillimeter Galaxies: Extended Star Formation in Massive High-redshift Galaxies., ApJ, 2009, vol. 699, p. 667

Murphy E. J., Chary R. R., Alexander D. M., Dickinson M., Magnelli B., Morrison G., Pope A., Teplitz H. I., Balancing the Energy Budget Between Star Formation and Active Galactic Nuclei in High-Redshift Infrared Luminous Galaxies., ApJ, 2009, vol. 698, p. 1380

Ota N., Nitrogen Substituted Polycyclic Aromatic Hydrocarbon As Capable Interstellar Infrared Spectrum Source Considering Astronomical Chemical Evolution Step To Biological Organic Purine And Adenine, ARXIV org., 2016, vol. 1603, p. 1

Papovich C., Moustakas L. A., Dickinson M., Le Floc'h E., Rieke G. H., Daddi E., Alexander D. M., Bauer F., Brandt W. N., Dahlen T., Egami E., Elbaz D., Ferguson H., Giavalisco M., et al. Spitzer Observations of Massive, Red Galaxies at High Redshift, ApJ, 2006, vol. 640, p. 92

Parker D. S. N., Kaiser R. I., O. K., Troy T., Ahmed M., Mebel A. M., Tielens A., GAS PHASE SYNTHESIS OF (ISO)QUINOLINE AND ITS ROLE IN THE FORMATION OF NUCLEOBASES IN THE INTERSTELLAR MEDIUM, ApJ, 2015, vol. 803, p. 53 
Parker D. S. N., Yang T., Dangi B. B., Kaiser R. I., Bera P. P., Lee T. J., LOW TEMPERATURE FORMATION OF NITROGEN-SUBSTITUTED POLYCYCLIC AROMATIC HYDROCARBONS (PANHs) - BARRIERLESS ROUTES TO DIHYDRO(iso)QUINOLINES, ApJ, 2015, vol. 815, p. 115

Peeters E., Hony S., Van Kerckkoven C., Tielens A. G. G. M., Allamandola L. J., Hudgins D. M., Bauschlicher C. W., The rich 6 to $9 \mu \mathrm{m}$ spectrum of interstellar PAHs., A\&A, 2002, vol. 390, p. 1089

Peeters Z., Botta O., Charnley S. B., Kisiel Z., Kuan Y., Ehrenfreund P., Formation and photostability of N-heterocycles in space, A\&A, 2005, vol. 433, p. 583

Pilleri P., Montillaud J., Berné O., Joblin C., Evaporating Very Small Grains as tracers of the UV radiation field in Photo-dissociation Regions, A\&A, 2012, vol. 542, p. A69

Pino T., Dartois E., Cao A.-T., Carpentier Y., Chamaillé T., Vasquez R., Jones A., d'Hendecourt L., Bréchignac P., The $6.2 \mu \mathrm{m}$ band position in laboratory and astrophysical spectra: a tracer of the aliphatic to aromatic evolution of interstellar carbonaceous dust, A\&A, 2008, vol. 490, p. 665

Pope A., Chary R.-R., Alexander D. M., Armus L., Dickinson M., Elbaz D., Frayer D., Scott D., Teplitz H., Mid-Infrared Spectral Diagnosis of Submillimeter Galaxies., ApJ, 2008, vol. 675 , p. 1171

Ricca A., Bauschlicher C. W., Bakes E. L. O., A Computational Study of the Mechanisms for the Incorporation of a Nitrogen Atom into Polycyclic Aromatic Hydrocarbons in the Titan Haze., Icarus, 2001, vol. 154, p. 516

Riechers D. A., Pope A., Daddi E., Armus L., Csrilli C. L., et al. Polycyclic Aromatic Hydrocarbon and Mid-Infrared Continuum Emission in a z i. 4 Submillimeter Galaxy, ApJ, 2014, vol. 786, p. 31

Robinson G., Smith R. G., Maldoni M., The water-ice librational band: radiative transfer model for AFGL961., MNRAS, 2012, vol. 424, p. 1530

Saladino R., Carota E., Botta G., Kapralov M., Timoshenko G. N., Rozanov A. Y., Krasavin E., Di Mauro E., Meteorite-catalyzed syntheses of nucleosides and of other prebiotic compounds from formamide underproton irradiation, PNAS, 2015, vol. 112, p. 2746 
Sales D. A., Pastoriza M. G., Riffel R., Winge C., Polycyclic Aromatic Hydrocarbon in the Central Region of the Seyfert 2 Galaxy NGC1808., MNRAS, 2012, vol. 429, p. 2634

Shi Y., Rieke G. H., Hines D. C., Gorjian V., Werner M. W., Cleary K., Low F. J., Smith P. S., Bouwman J., $9.7 \mu m$ Silicate Features in Active Galactic Nuclei: New Insights into Unification Models., ApJ, 2006, vol. 675, p. 960

Siana B., Smail I., Swinbank A., Richard J., Teplitz H., Coppin K., Ellis R., Kneib J.P., Edge A., DETECTION OF FAR-INFRARED AND POLYCYCLIC AROMATIC HYDROCARBON EMISSION FROM THE COSMIC EYE: PROBING THE DUST AND STAR FORMATION OF LYMAN BREAK GALAXIES, ApJ, 2009, vol. 698, p. 1273

Smith J. D. T., Draine B., Dale D. A., et al. THE MID-INFRARED SPECTRUM OF STAR-FORMING GALAXIES: GLOBAL PROPERTIES OF POLYCYCLIC AROMATIC HYDROCARBON EMISSION, ApJ, 2007, vol. 656, p. 770

Spoon H. W. W., Keane J. V., Tielens A. G. G. M., Lutz D., Moorwood F. M., The obscured mid-infrared continuum of NCG4418: A dust- and ice-enshrouded AGN., A\&A, 2001, vol. 365, p. L353

Spoon H. W. W., Keane J. V., Tielens A. G. G. M., Lutz D., Moorwood F. M., Laurent O., Ice features in the mid-IR spectra of galactic nuclei., A\&A, 2002, vol. 385, p. 1022

Sturm E., Hasinger G., Lehmann I., Mainieri V., Genzel R., Lehnert M. D., Lutz D., Tacconi L. J., Mid-Infrared Spitzer Spectra of X-Ray-Selected Type 2 QSOs: QSO2s Are Not Ultraluminous Infrared Galaxies., ApJ, 2006, vol. 642, p. 81

Teplitz H., Desai V., Armus L., Chary R., Marshall J., Colbert J., Frayer D., Pope A., Blain A., Spoon H., Charmandaris V., Scott D., Measuring PAH Emission in Ultradeep Spitzer IRS Spectroscopy of High Redshift IR Luminous Galaxies, ApJ, 2007, vol. 659, p. 941

Tielens A. G. G. M., The Physics and Chemistry of the Interstellar Medium. Cambridge University Press, 2005, 510 p. 
Tielens A. G. G. M., Interstellar Polycyclic Aromatic Hydrocarbon Molecules, Annu. Rev. Astro. Astrophys., 2008, vol. 46, p. 289

Tielens A. G. G. M., The molecular universe., REVIEWS OF MODERN PHYSICS, 2013, vol. 85

Van Diedenhoven B., Peeters E., Van Kerckhoven C., Hony S., Hudgins D. M., Allamandola L. J., Tielens A. G. G. M., The Profiles of the 3-12 Micron Polycyclic Aromatic Hydrocarbon Features, ApJ, 2004, vol. 611, p. 928

Weedman D., Polletta M., Lonsdale C. J., Wilkes B. J., Siana B., Houck J. R., Surace J., Shupe D., Farrah D., Smith H. E., Active Galactic Nucleus and Starburst Classification from Spitzer Mid-Infrared Spectra for High-Redshift SWIRE Sources., ApJ, 2006, vol. 653 , p. 101

Weedman D. W., Hao L., Higdon S. J. U., Devost D., Wu Y., Charmandaris V., Brandl B., Bass E., Houck J. R., Mid-Infrared Spectra of Classical AGNs Observed with the Spitzer Space Telescope., ApJ, 2005, vol. 633, p. 706

Weedman D. W., Houck J. R., Average Infrared Galaxy Spectra from Spitzer Flux-Limited Samples., ApJ, 2009, vol. 693, p. 370

Werner M. W., Roellig T. L., Low F. J., Rieke G. H., Rieke M., Hoffmann W. F., Young E., Houck J. R., Brandl B., Fazio G. G., Hora J. L., Gehrz R. D., Helou G., Soifer B. T., et al. The Spitzer Space Telescope Mission., The Astrophysical Journal Supplement Series, 2004, vol. 154, p. Issue 1, 1

Wu Y., Charmandaris V., Huang J., Spinoglio L., Tommasin S., Spitzer/IRS $5-35 \mu m$ Low-resolution Spectroscopy of the $12 \mu m$ Seyfert Sample., ApJ, 2009, vol. 701, p. 658

Wu Y., Helou G., Armus L., Cormier D., Shi Y., Dale D., Dasyra K., Smith J. D., Papovich C., et al. INFRARED LUMINOSITIES AND AROMATIC FEATURES IN THE $24 \mu \mathrm{m}$ FLUX-LIMITED SAMPLE OF 5MUSES, ApJ, 2010, vol. 723, p. 895

Yan L., Chary R., Armus L., Teplitz H., Helou G., Frayer D., Fadda D., Surace J., Choi P., Spitzer Detection of Polycyclic Aromatic Hydrocarbon and Silicate Dust Features in 
the Mid-Infrared Spectra of z 2 Ultraluminous Infrared Galaxies, ApJ, 2005, vol. 628, p. 604

Yan L., Sajina A., Fadda D., Choi P., Armus L., Helou G., Teplitz H., Frayer D., Surace J., SPITZER MID-INFRARED SPECTROSCOPY OF INFRARED LUMINOUS GALAXIES AT z 2. I.THE SPECTRA, ApJ, 2007, vol. 658, p. 778

Zakamska N. L., Gómez L., Strauss M. A., Krolik J. H., Mid-Infrared Spectra of OpticallySelected Type 2 Quasars., AJ, 2008, vol. 136, p. 160 
Apêndice 



\section{Apêndice A}

\section{Lista de abreviações}

AGB Da sigla em inglês para Asymptotic giant branch - Ramo assimptótico das gigantes.

AGN Da sigla em inglês para Active Galactic Nucleus - Núcleo ativo de galáxia.

AIB Da sigla em inglês para Aromatic Infrared Band - Banda Aromática no Infravermelho.

AORKEY Da sigla em inglês para Astronomical Observation Request key.

BCD Sigla em inglês para 2D Basic Calibrated Data.

DNA Da sigla em inglês para deoxyribonucleic acid - ácido desoxirribonucleico.

eVSG Da sigla em inglês para evaporating Very Small Grains - grãos muito pequenos em evaporação.

FHWM Da sigla em inglês para Full Width at Half Maximum - largura à meia altura.

HACA Da sigla em inglês para Hydrogen-Abstraction aCetylene-Addition - Abstração de hidrogênio e adição de acetileno.

IDL Sigla em inglês para Interactive Data Language.

IR Infravermelho.

IRAC Da sigla em inglês para Infrared Array Camera - Rede de câmeras do infravermelho, instrumento a bordo do telescópio Spitzer.

IRAF Sigla em inglês para Image Reduction and Analysis Facility.

IRS Da sigla em inglês para Infrared Spectrograph - Espectrógrafo de infravermelho, instrumento a bordo do telescópio Spitzer.

IRSA Sigla em inglês para NASA/IPAC Infrared Science Archives.

ISM Sigla em inglês para Interstellar Medium - meio interestelar.

JWST Sigla em inglês paraJames Webb Space Telescope - Telescópio Espacial James 
Webb.

MIPS Da sigla em inglês para Multiband Imaging Photometer for Spitzer - Fotômetro de imagem de multibanda para o Spitzer.

MIR ou mid-IR Da sigla em inglês para Mid Infrared - infravermelho médio.

NED Da sigla em inglês para NASA Extragalactic Data base - base de dados extragalácticos da NASA.

PAH Da sigla em inglês para polycyclic aromatic hydrocarbons - hidrocarbonetos policíclicos aromáticos.

PANH Da sigla em inglês para polycyclic aromatic nitrogen heterocycles - heterociclos policíclicos aromáticos nitrogenados.

RMS Da sigla em inglês para root mean square - raiz do valor quadrático médio.

RNA Da sigla em inglês para ribonucleic acid - ácido ribonucleico.

SHA Sigla em inglês para Spitzer Heritage Archive.

SSC Sigla em inglês para Spitzer Science Center.

ULIRG Da sigla em inglês para Ultra Luminous Infrared Galaxy - galáxia no infravermelho ultra luminosa.

UV Ultravioleta.

VSG Da sigla em inglês para Very Small Grains - grãos muito pequenos. 


\section{Apêndice B}

\section{Espectros decompostos pelo PAHFIT e seus respectivos RMS}

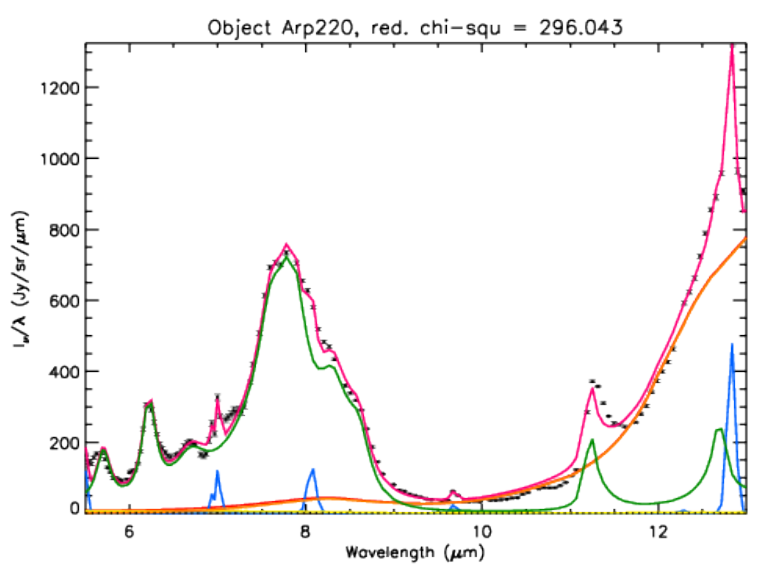

Figura B.1: Espectro do objeto Arp220 decomposto com o PAHFIT e o valor do $\chi^{2}$ reduzido.

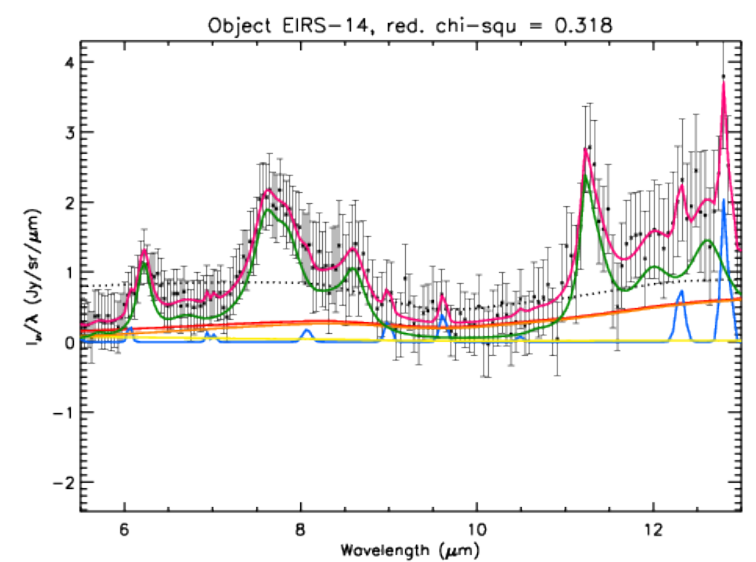

Figura B.3: Espectro do objeto EIRS-14 decomposto com o PAHFIT e o valor do $\chi^{2}$ reduzido.

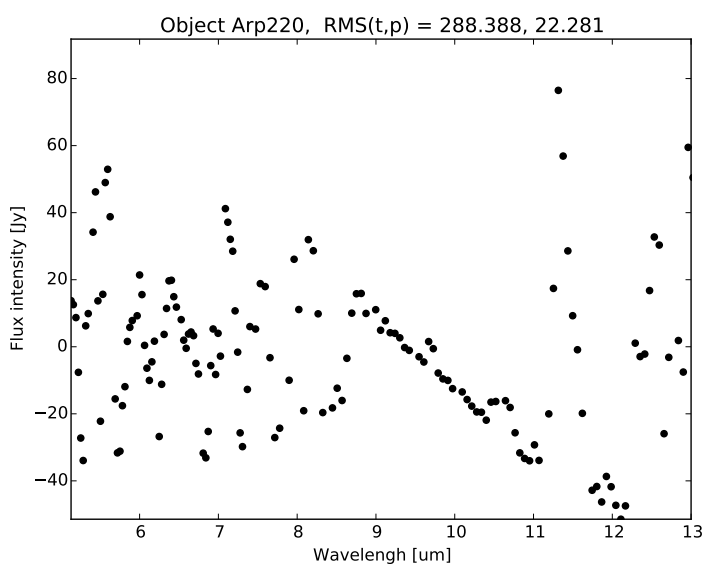

Figura B.2: Gráfico de RMS do objeto Arp220 com os valores total e parcial.

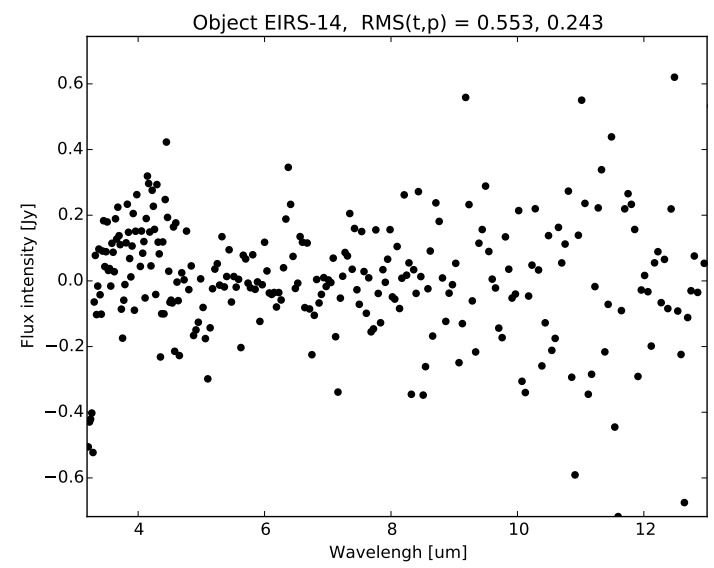

Figura B.4: Gráfico de RMS do objeto EIRS-14 com os valores total e parcial. 


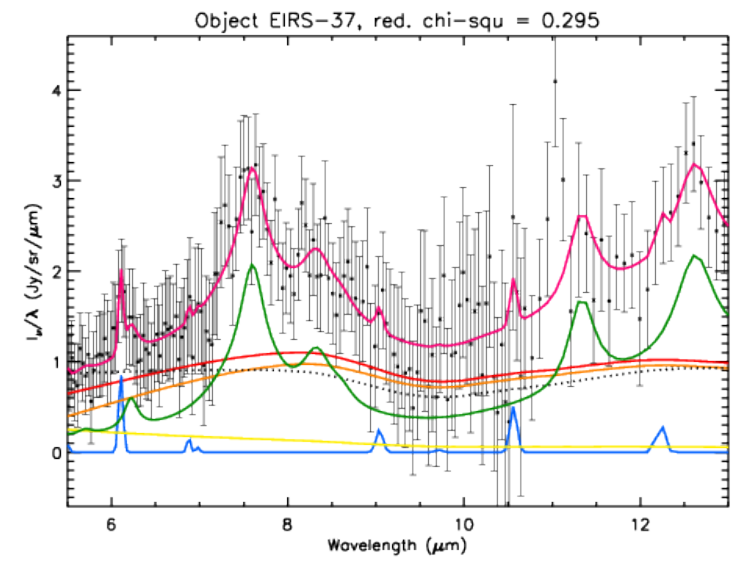

Figura B.5: Espectro do objeto EIRS-37 decomposto com o PAHFIT e o valor do $\chi^{2}$ reduzido.

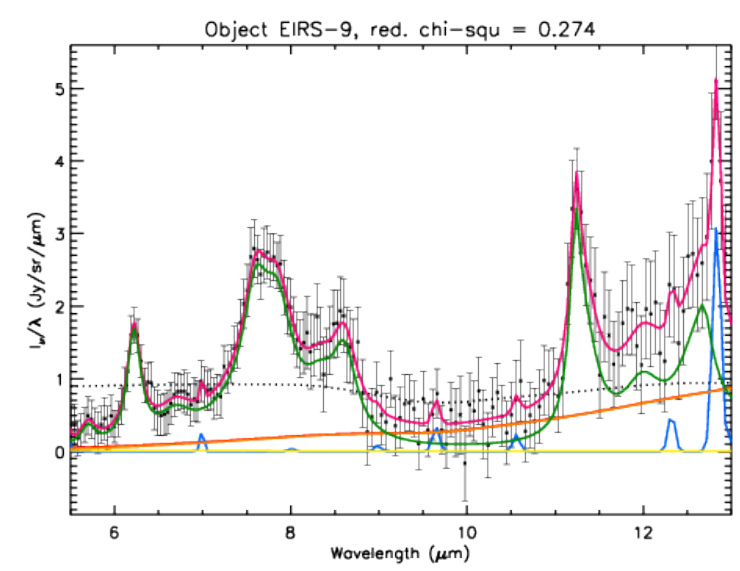

Figura B.7: Espectro do objeto EIRS-9 decomposto com o PAHFIT e o valor do $\chi^{2}$ reduzido.

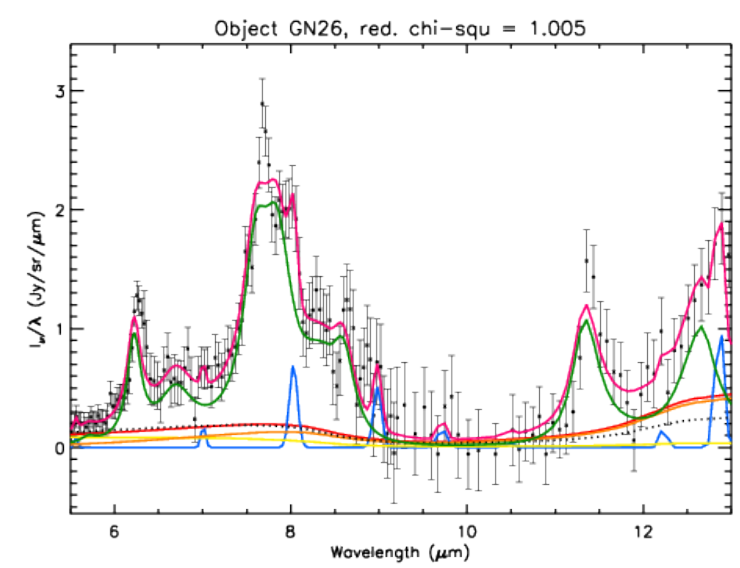

Figura B.9: Espectro do objeto GN26 decomposto com o PAHFIT e o valor do $\chi^{2}$ reduzido.

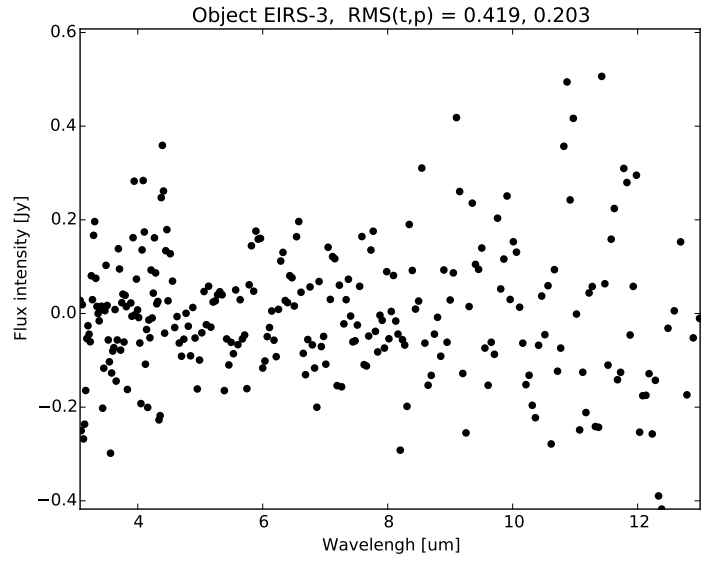

Figura B.6: Gráfico de RMS do objeto EIRS-37 com os valores total e parcial.

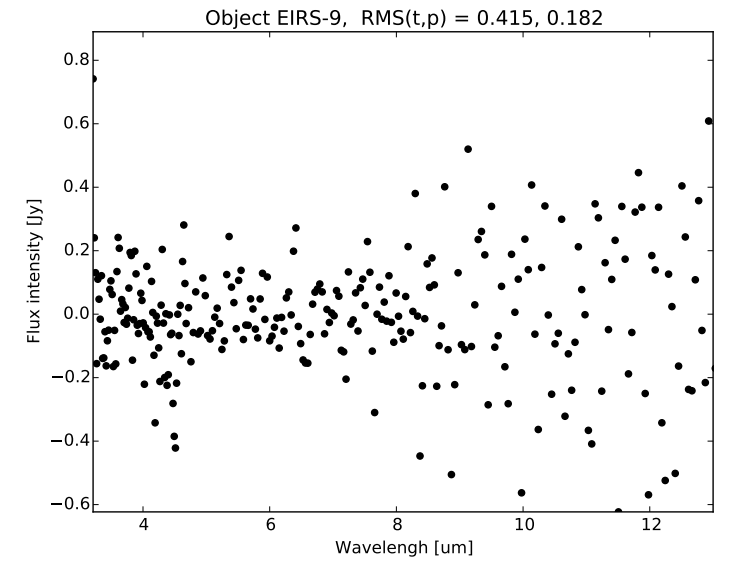

Figura B.8: Gráfico de RMS do objeto EIRS-9 com os valores total e parcial.

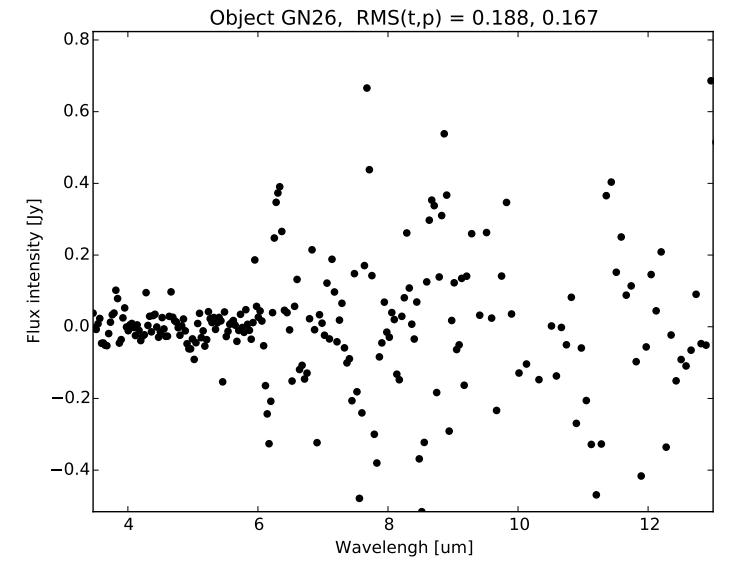

Figura B.10: Gráfico de RMS do objeto GN26 com os valores total e parcial. 


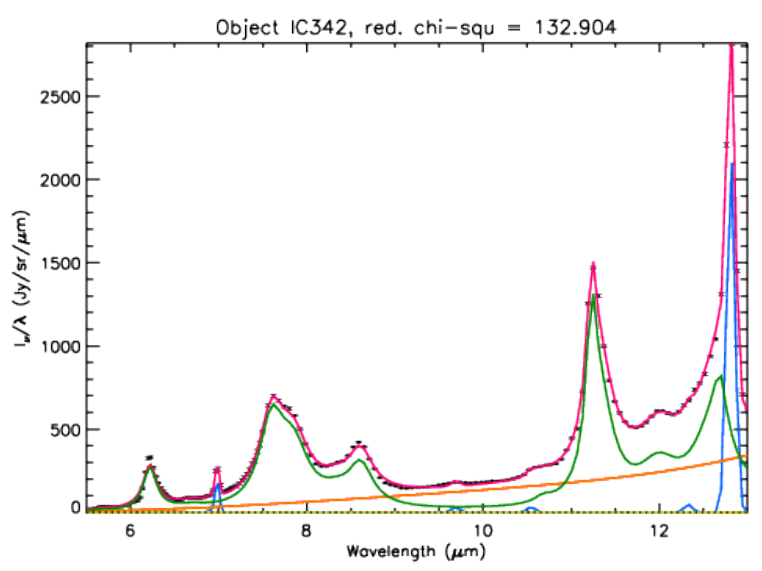

Figura B.11: Espectro do objeto IC342 decomposto com o PAHFIT e o valor do $\chi^{2}$ reduzido.

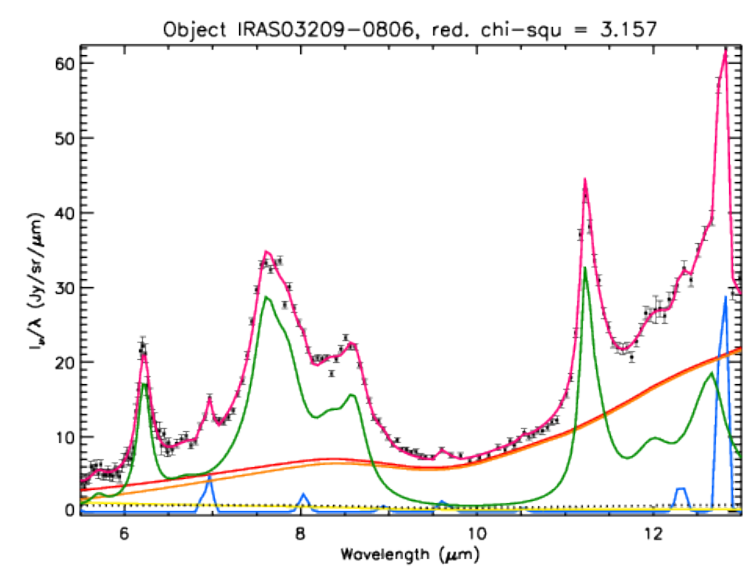

Figura B.13: Espectro do objeto IRAS03209-0806 decomposto com o PAHFIT e o valor do $\chi^{2}$ reduzido.

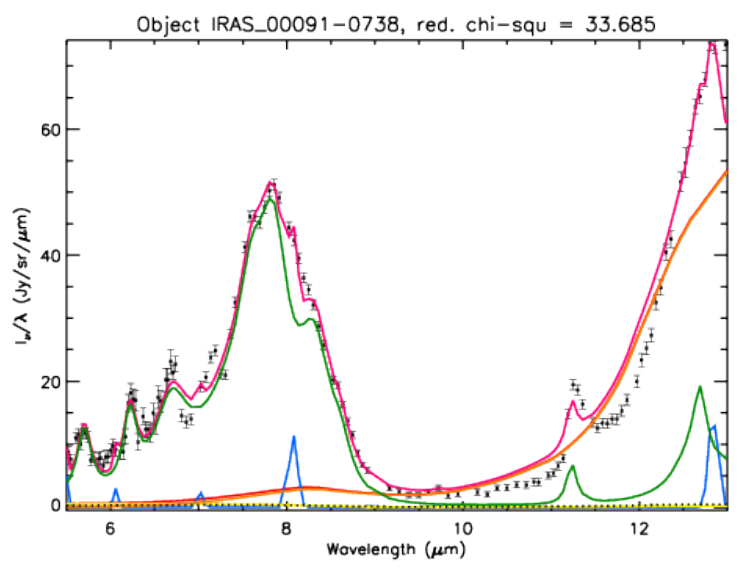

Figura B.15: Espectro do objeto IRAS_000910738 decomposto com o PAHFIT e o valor do $\chi^{2}$ reduzido.

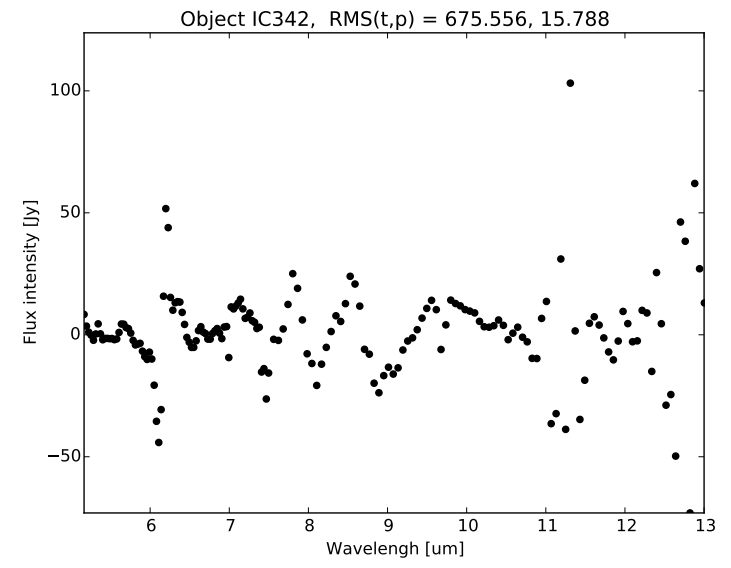

Figura B.12: Gráfico de RMS do objeto IC342 com os valores total e parcial.

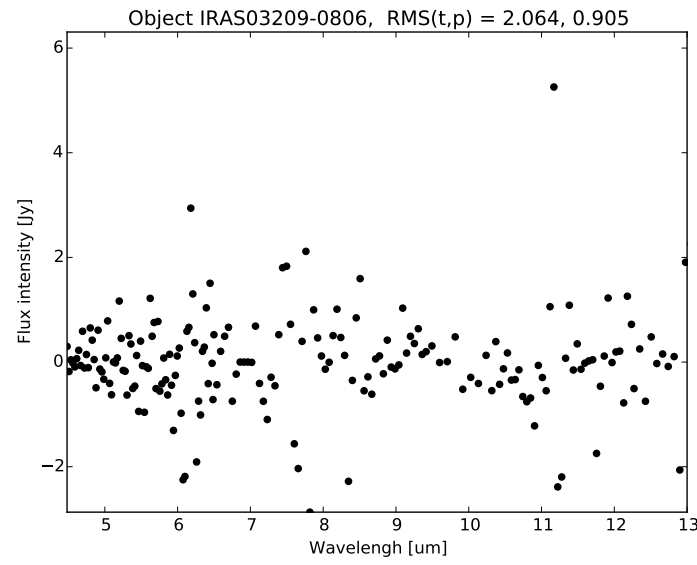

Figura B.14: Gráfico de RMS do objeto IRAS03209-0806 com os valores total e parcial.

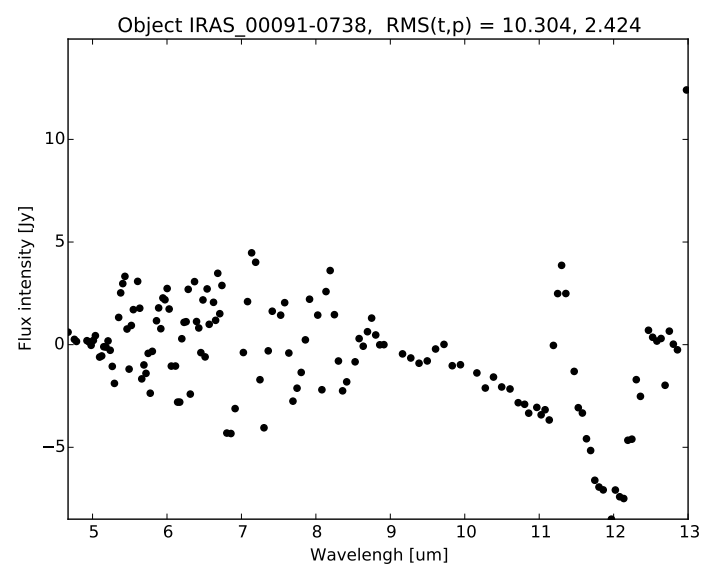

Figura B.16: Gráfico de RMS do objeto IRAS_00091-0738 com os valores total e parcial. 


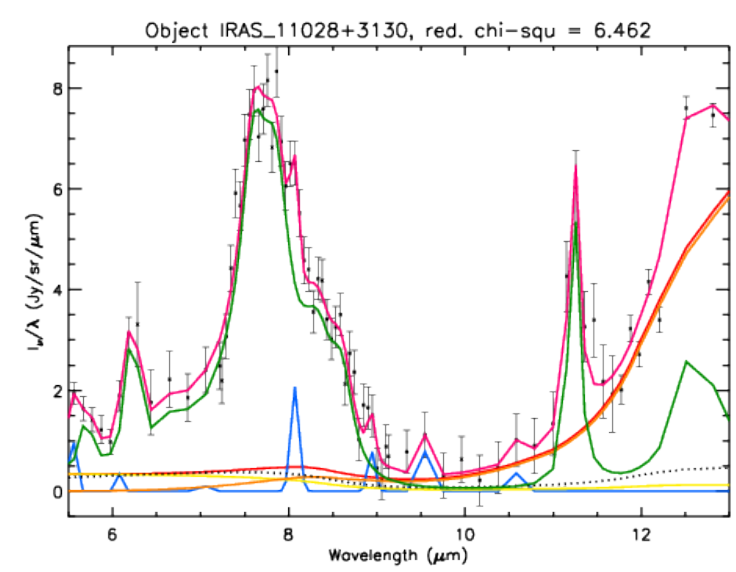

Figura B.17: Espectro do objeto IRAS_11028+3130 decomposto com o PAHFIT e o valor do $\chi^{2}$ reduzido.

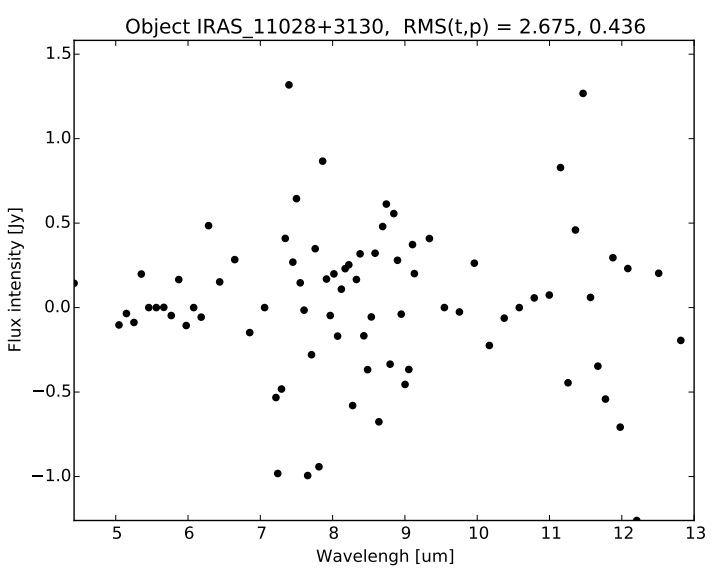

Figura B.18: Gráfico de RMS do objeto IRAS_11028+3130 com os valores total e parcial.

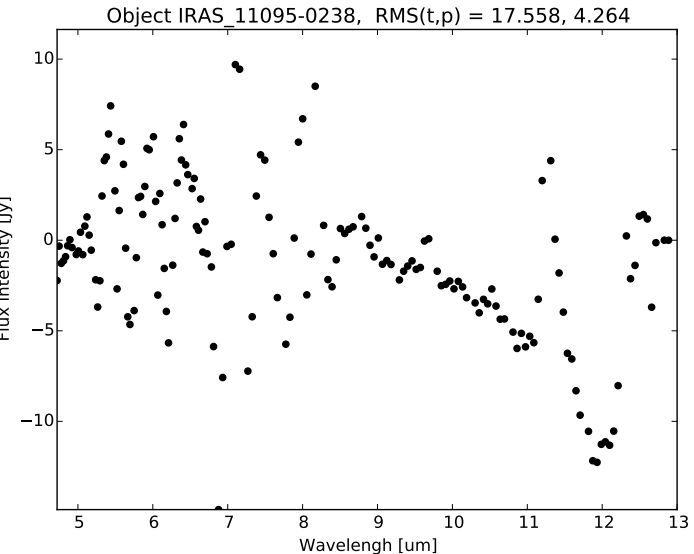

Figura B.20: Gráfico de RMS do objeto IRAS_11095-0238 com os valores total e parcial. 0238 decomposto com o PAHFIT e o valor do $\chi^{2}$ reduzido. 


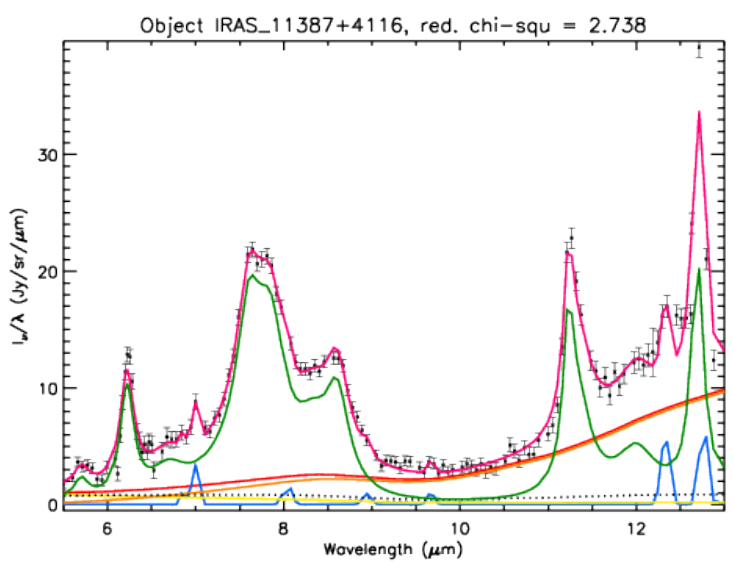

Figura B.21: Espectro do objeto IRAS_11387+4116 decomposto com o PAHFIT e o valor do $\chi^{2}$ reduzido.

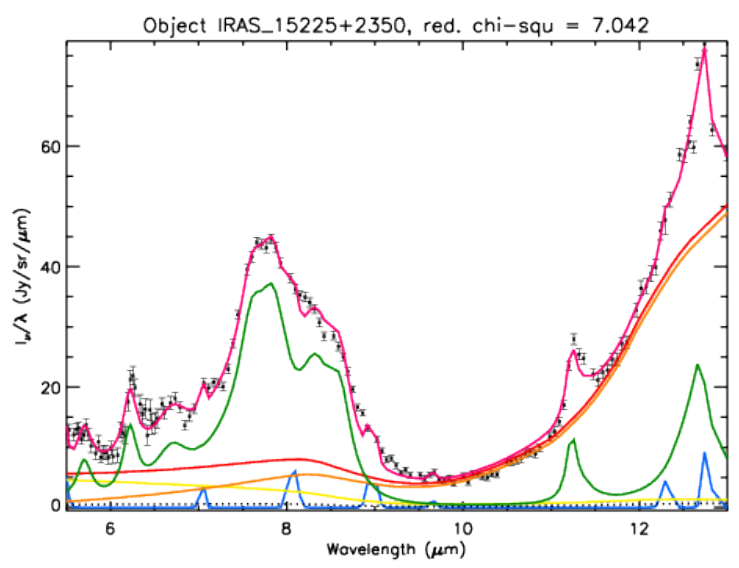

Figura B.23: Espectro do objeto IRAS_15225+2350 decomposto com o PAHFIT e o valor do $\chi^{2}$ reduzido.

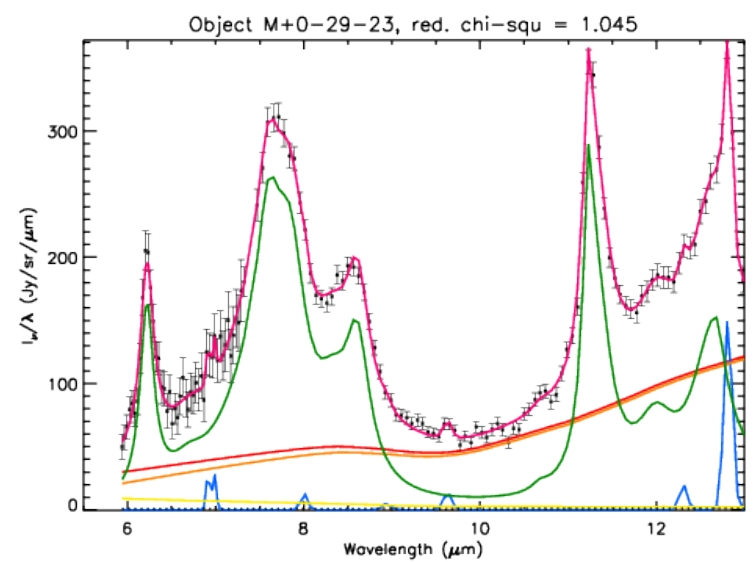

Figura B.25: Espectro do objeto $\mathrm{M}+0-29-23$ decomposto com o PAHFIT e o valor do $\chi^{2}$ reduzido.

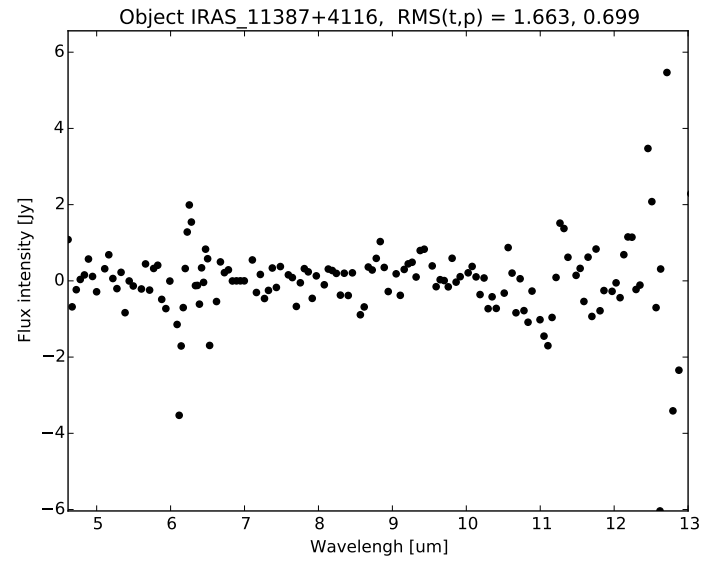

Figura B.22: Gráfico de RMS do objeto IRAS_11387+4116 com os valores total e parcial.

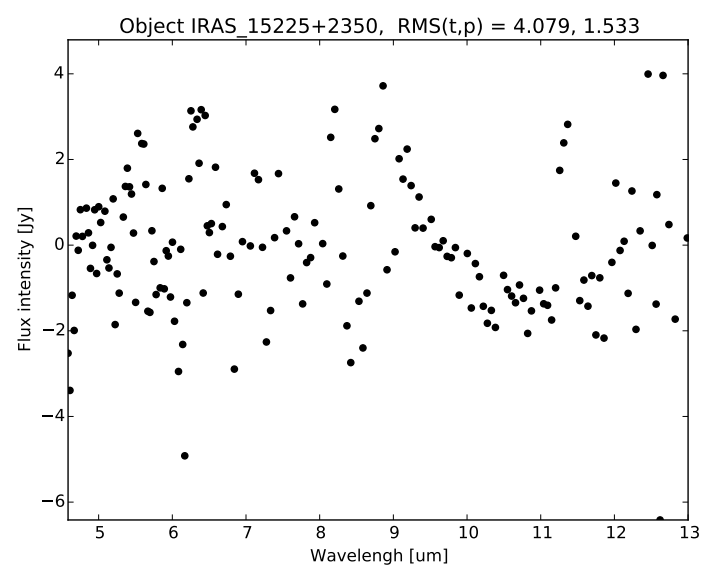

Figura B.24: Gráfico de RMS do objeto IRAS_15225+2350 com os valores total e parcial.

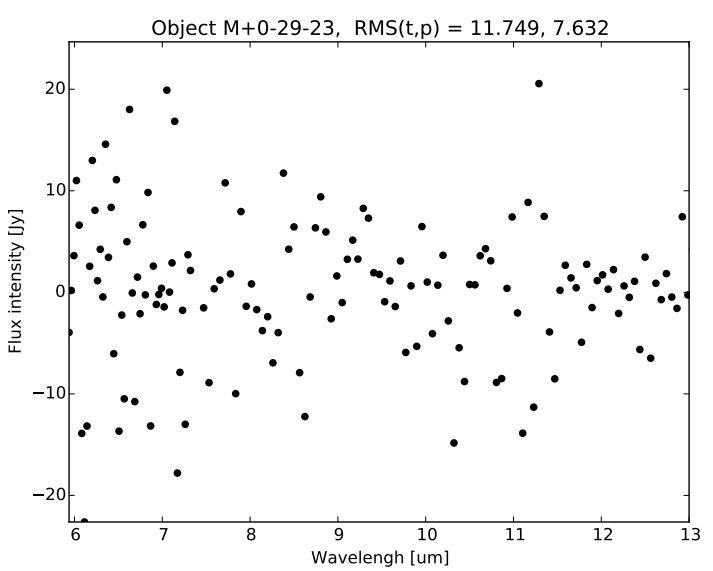

Figura B.26: Gráfico de RMS do objeto M+0-2923 com os valores total e parcial. 


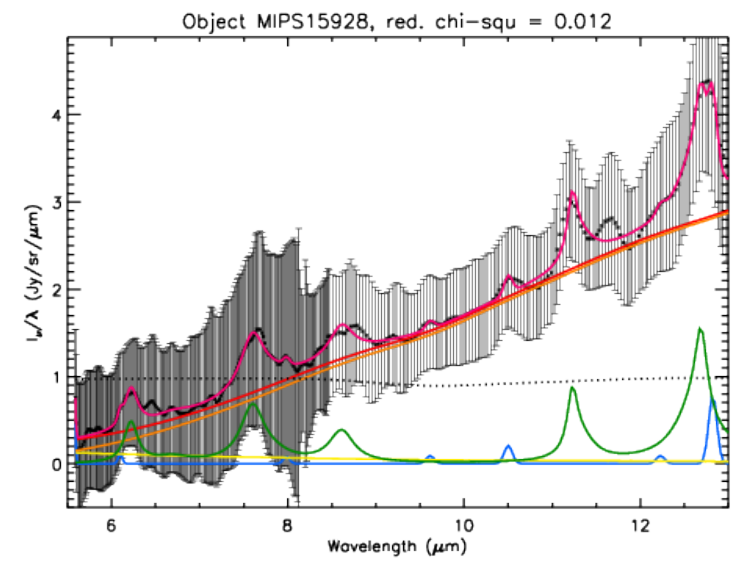

Figura B.27: Espectro do objeto MIPS15928 decomposto com o PAHFIT e o valor do $\chi^{2}$ reduzido.

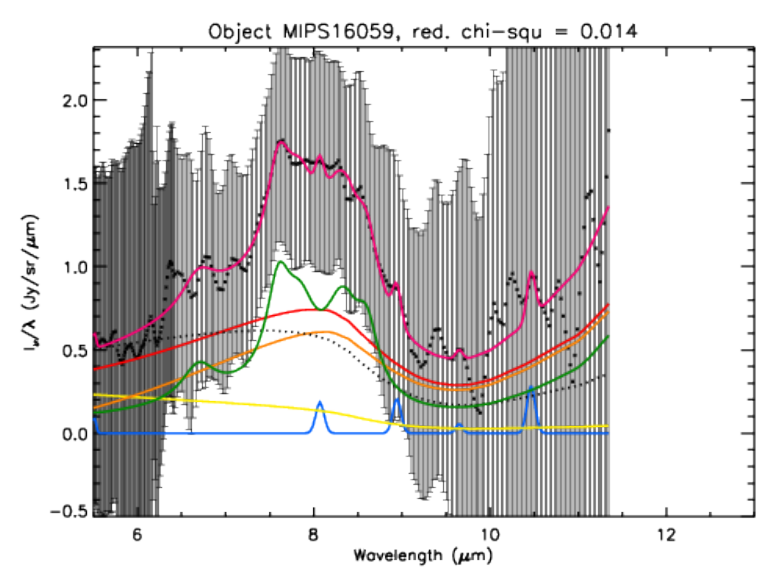

Figura B.29: Espectro do objeto MIPS16059 decomposto com o PAHFIT e o valor do $\chi^{2}$ reduzido.

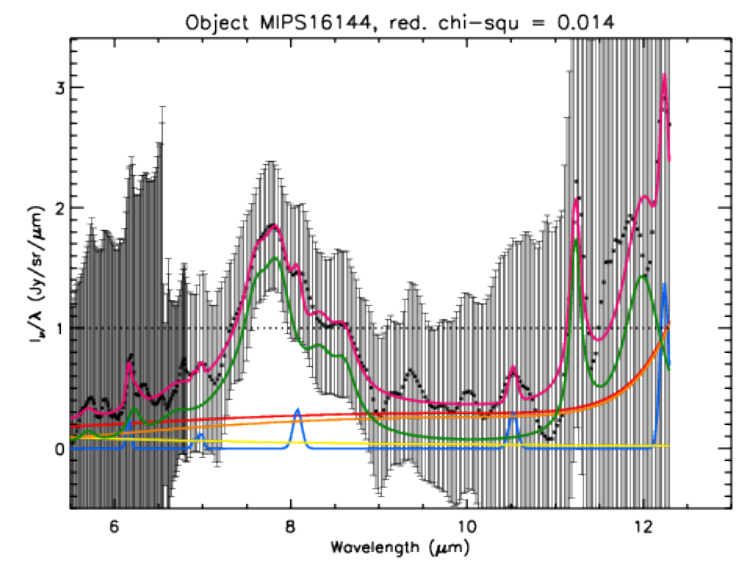

Figura B.31: Espectro do objeto MIPS16144 decomposto com o PAHFIT e o valor do $\chi^{2}$ reduzido.

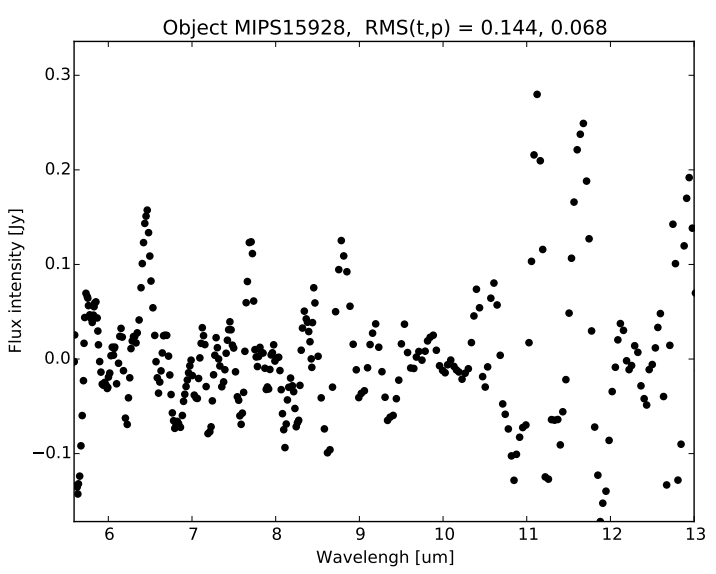

Figura B.28: Gráfico de RMS do objeto MIPS15928 com os valores total e parcial.

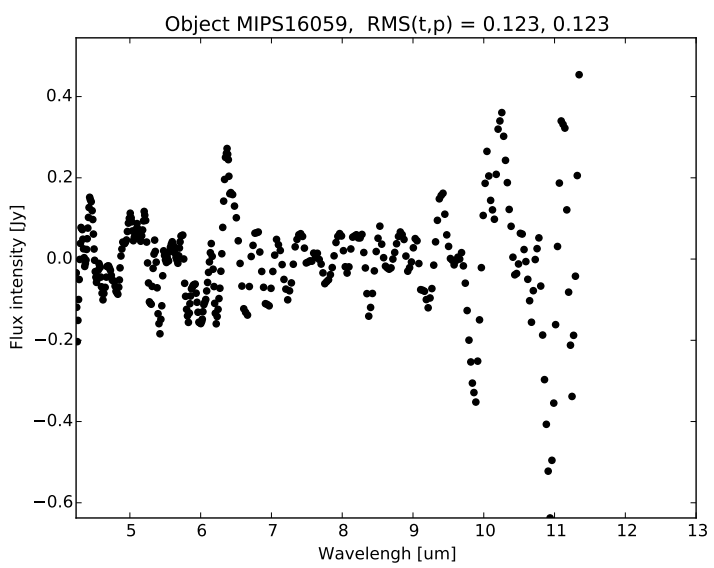

Figura B.30: Gráfico de RMS do objeto MIPS16059 com os valores total e parcial.

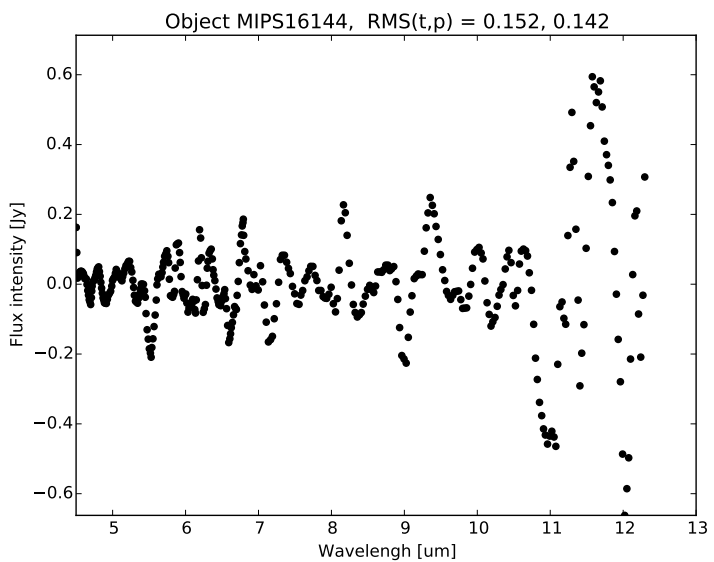

Figura B.32: Gráfico de RMS do objeto MIPS16144 com os valores total e parcial. 


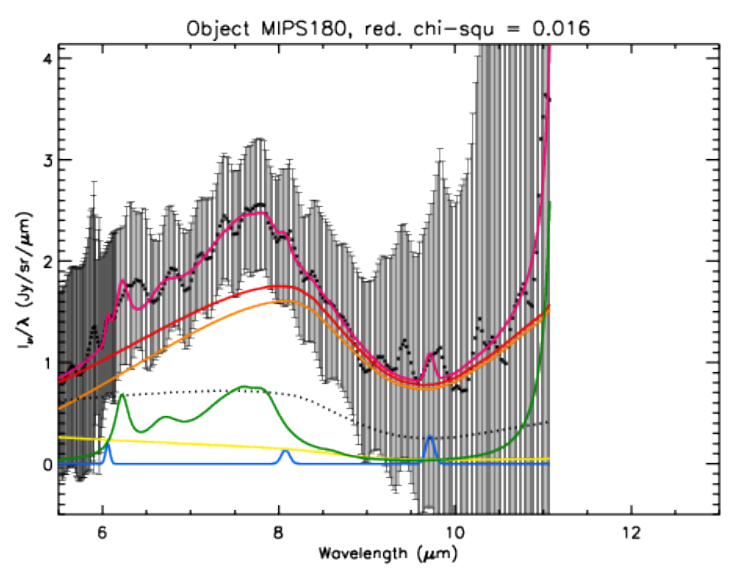

Figura B.33: Espectro do objeto MIPS180 decomposto com o PAHFIT e o valor do $\chi^{2}$ reduzido.

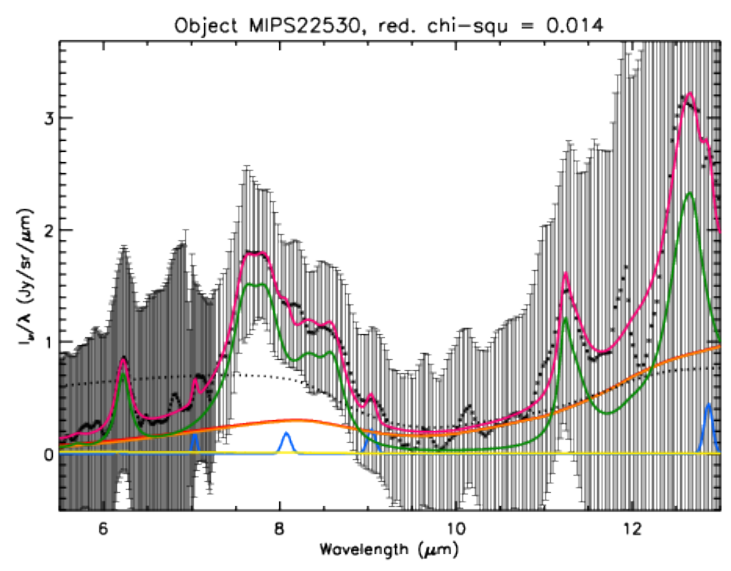

Figura B.35: Espectro do objeto MIPS22530 decomposto com o PAHFIT e o valor do $\chi^{2}$ reduzido.

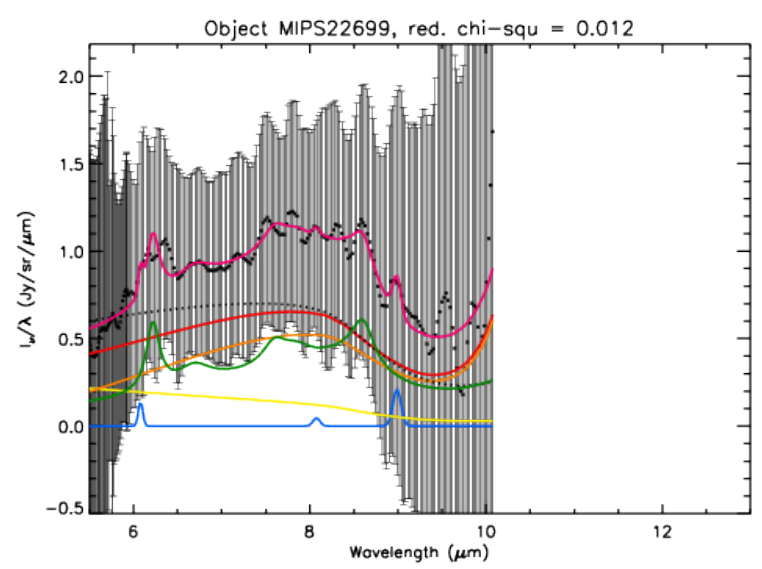

Figura B.37: Espectro do objeto MIPS22699 decomposto com o PAHFIT e o valor do $\chi^{2}$ reduzido.

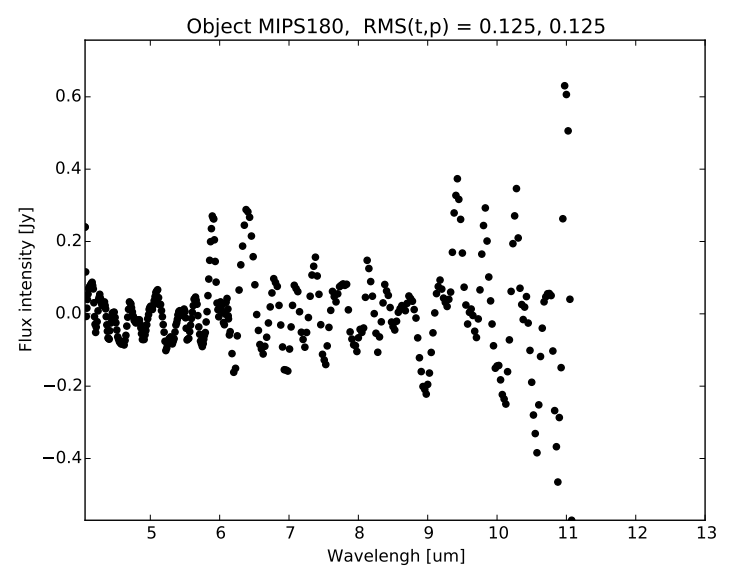

Figura B.34: Gráfico de RMS do objeto MIPS180 com os valores total e parcial.

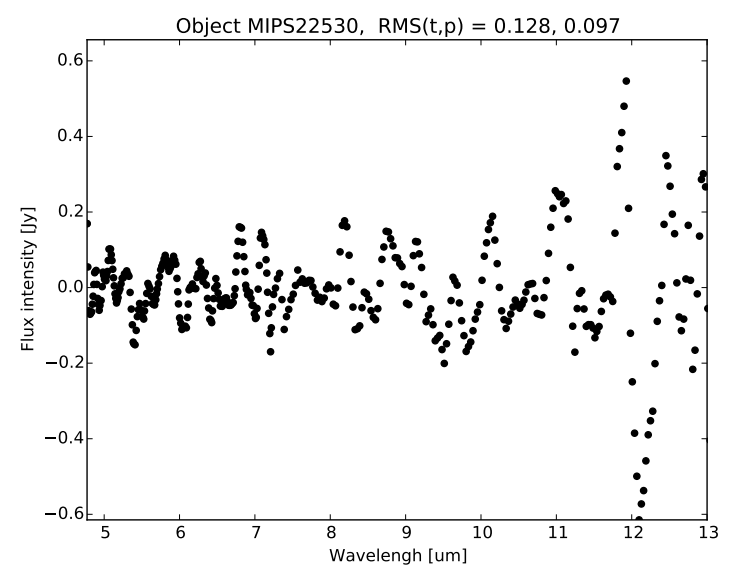

Figura B.36: Gráfico de RMS do objeto MIPS22530 com os valores total e parcial.

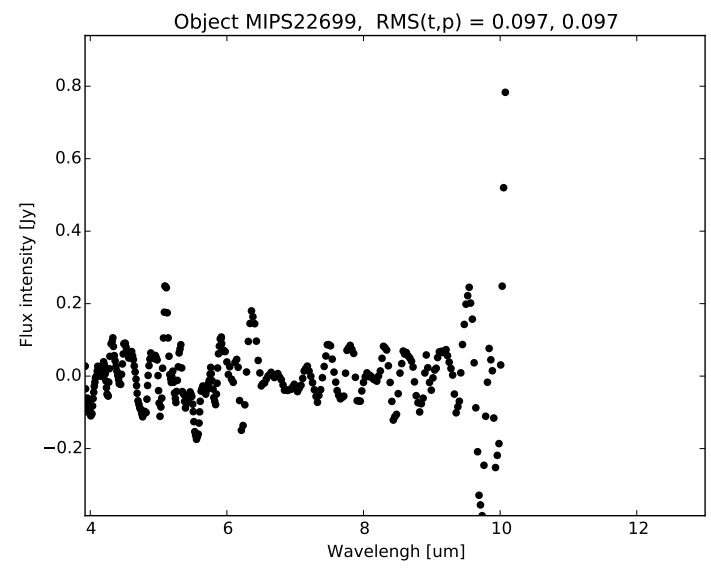

Figura B.38: Gráfico de RMS do objeto MIPS22699 com os valores total e parcial. 


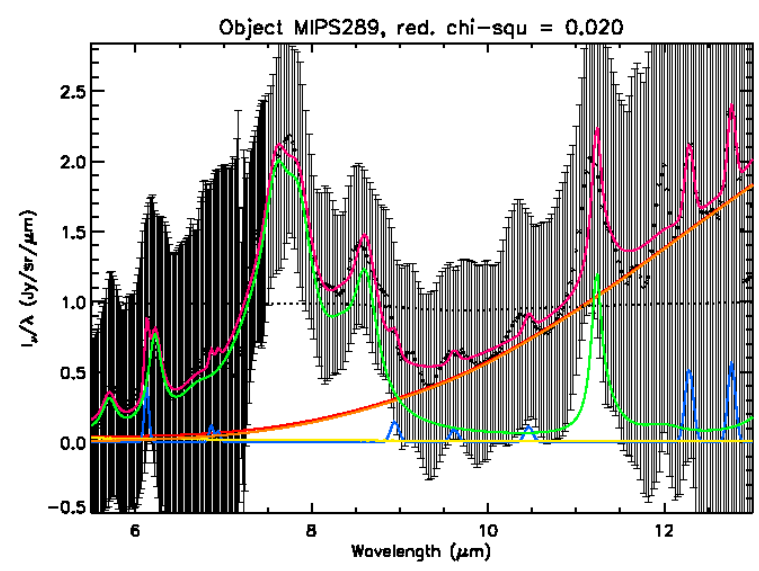

Figura B.39: Espectro do objeto MIPS289 decomposto com o PAHFIT e o valor do $\chi^{2}$ reduzido.

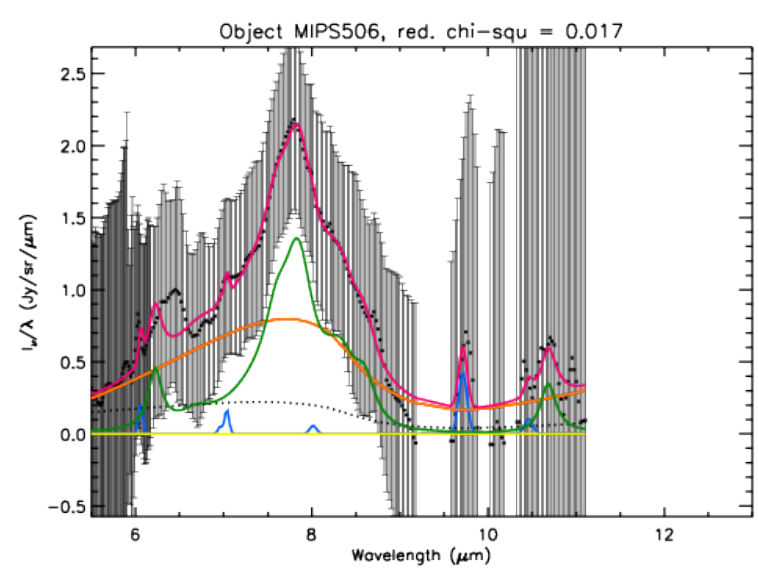

Figura B.41: Espectro do objeto MIPS506 decomposto com o PAHFIT e o valor do $\chi^{2}$ reduzido.

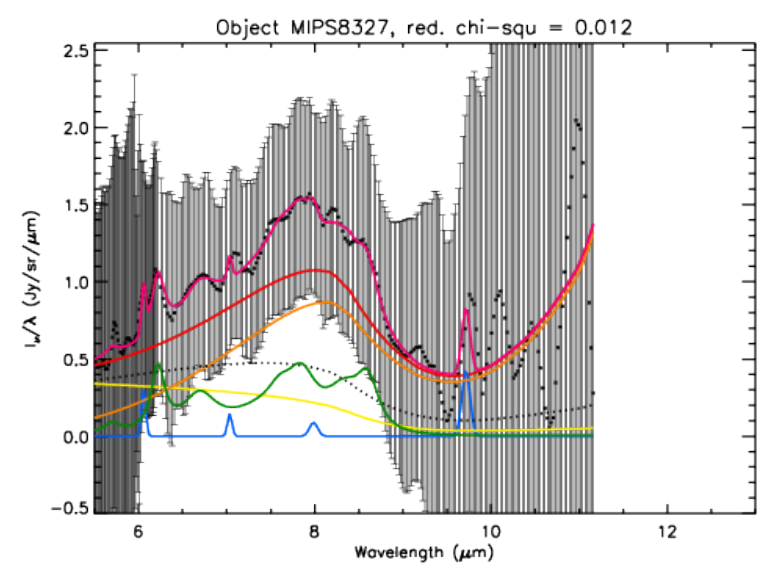

Figura B.43: Espectro do objeto MIPS8327 decomposto com o PAHFIT e o valor do $\chi^{2}$ reduzido.

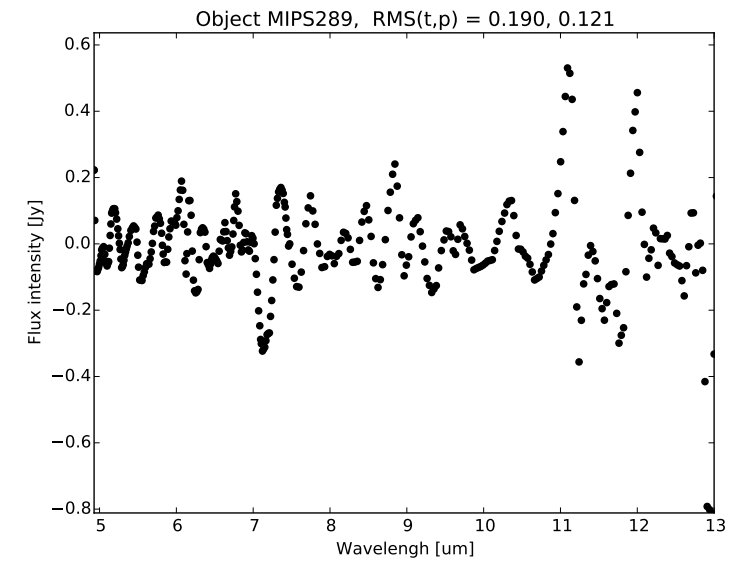

Figura B.40: Gráfico de RMS do objeto MIPS289 com os valores total e parcial.

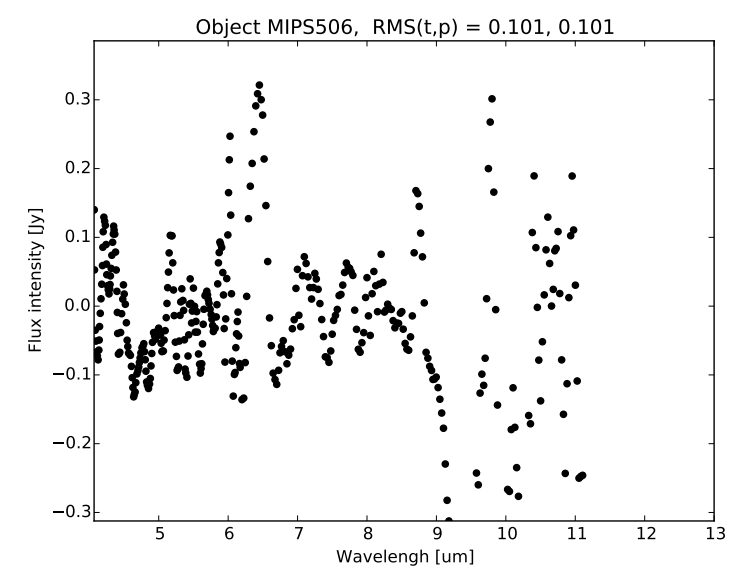

Figura B.42: Gráfico de RMS do objeto MIPS506 com os valores total e parcial.

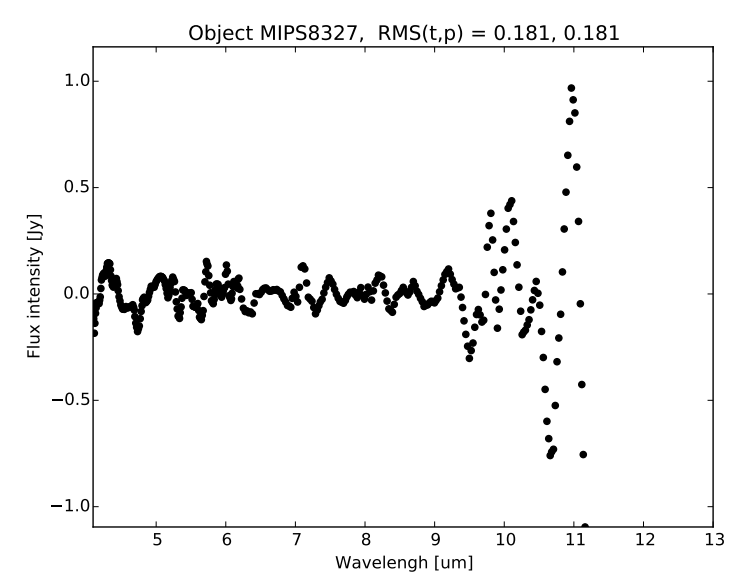

Figura B.44: Gráfico de RMS do objeto MIPS8327 com os valores total e parcial. 


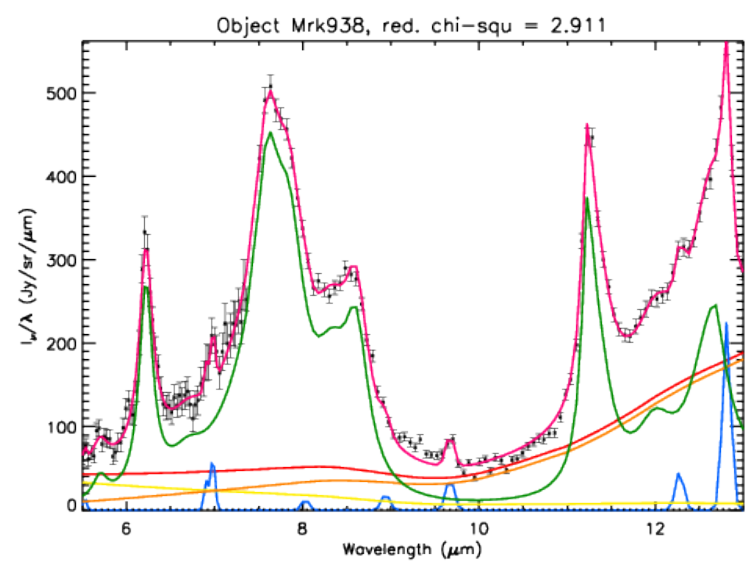

Figura B.45: Espectro do objeto Mrk938 decomposto com o PAHFIT e o valor do $\chi^{2}$ reduzido.

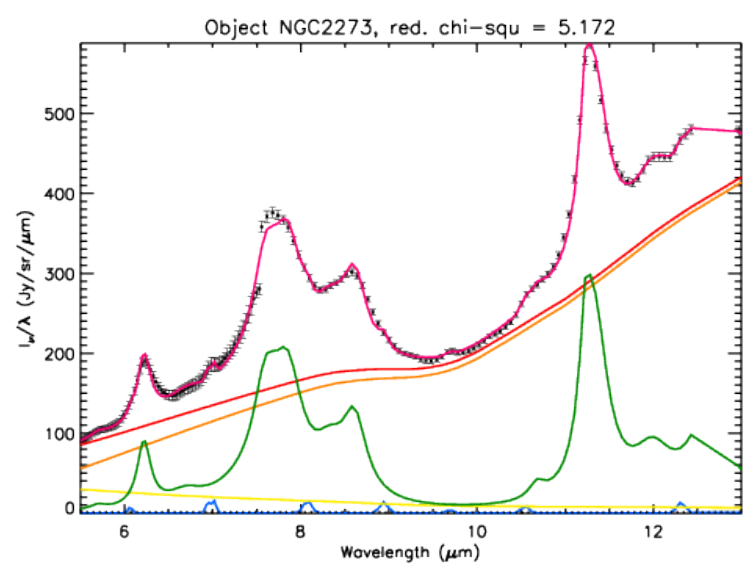

Figura B.47: Espectro do objeto NGC2273 decomposto com o PAHFIT e o valor do $\chi^{2}$ reduzido.

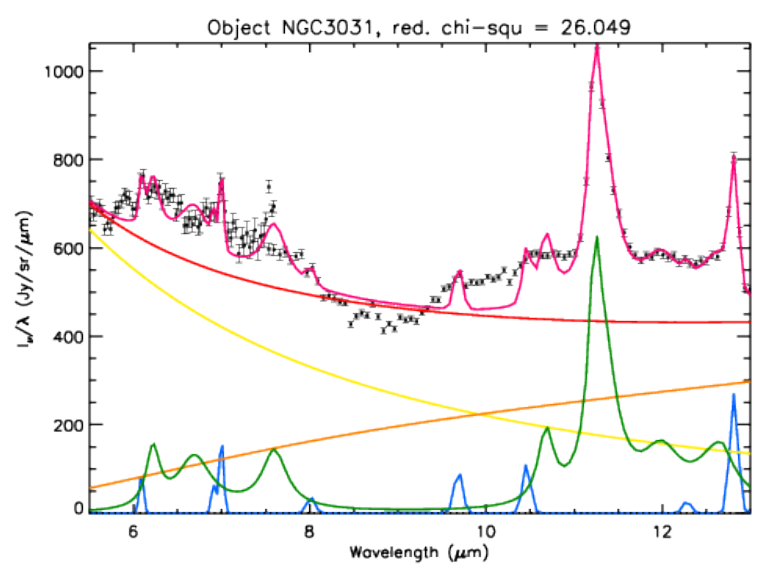

Figura B.49: Espectro do objeto NGC3031 decomposto com o PAHFIT e o valor do $\chi^{2}$ reduzido.

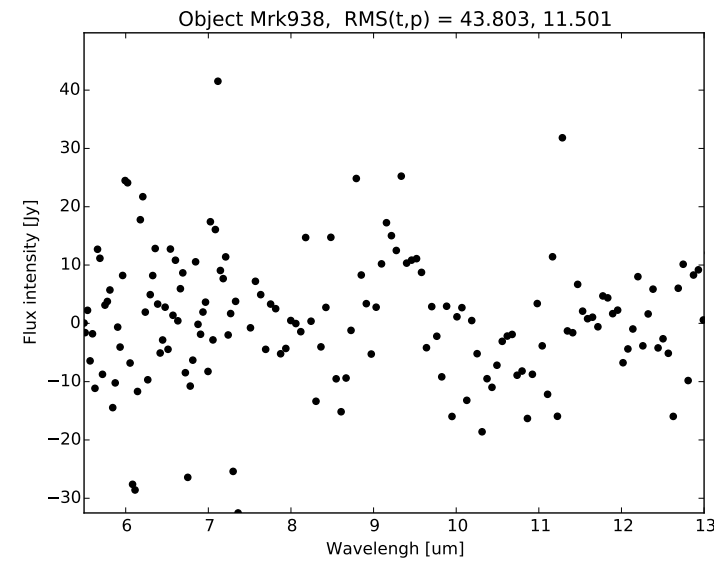

Figura B.46: Gráfico de RMS do objeto Mrk938 com os valores total e parcial.

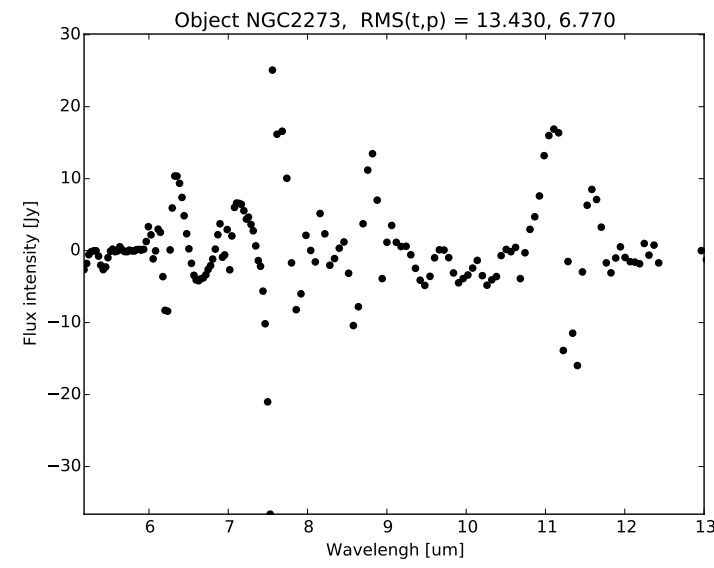

Figura B.48: Gráfico de RMS do objeto NGC2273 com os valores total e parcial.

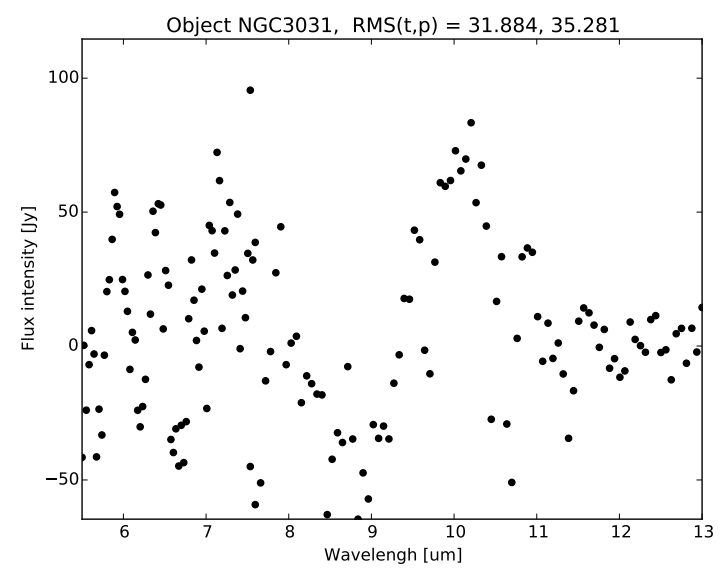

Figura B.50: Gráfico de RMS do objeto NGC3031 com os valores total e parcial. 


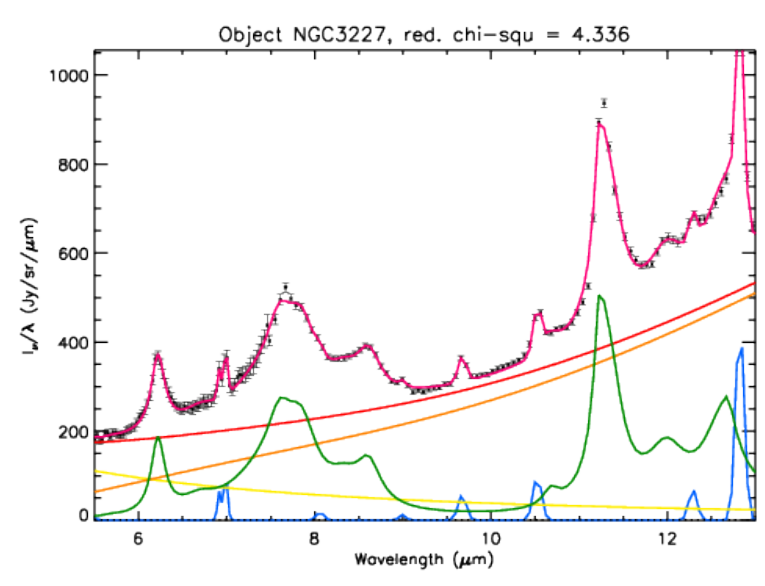

Figura B.51: Espectro do objeto NGC3227 decomposto com o PAHFIT e o valor do $\chi^{2}$ reduzido.

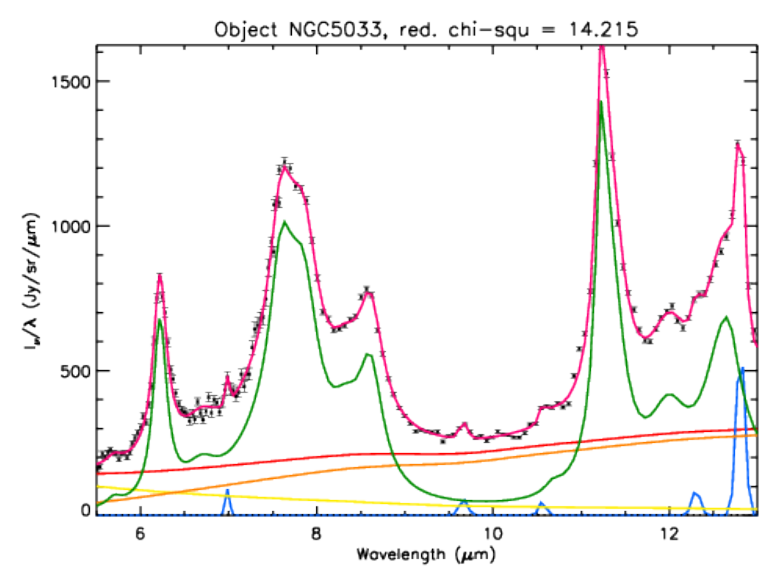

Figura B.53: Espectro do objeto NGC5033 decomposto com o PAHFIT e o valor do $\chi^{2}$ reduzido.

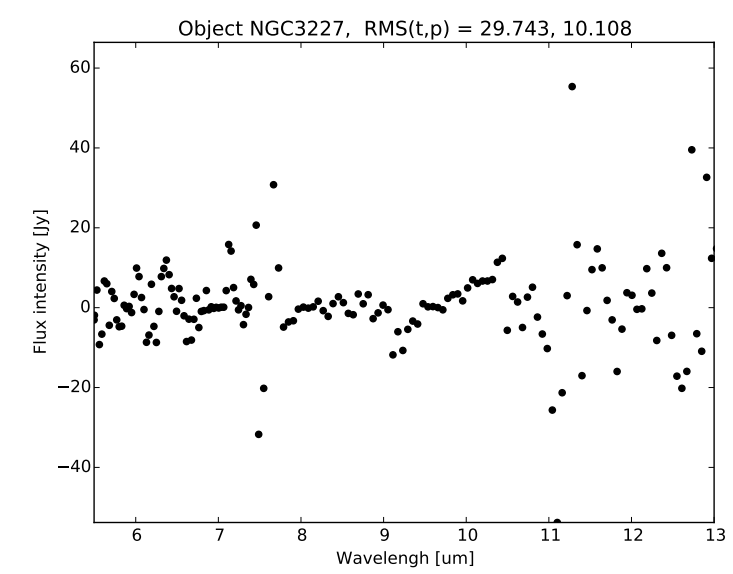

Figura B.52: Gráfico de RMS do objeto NGC3227 com os valores total e parcial.

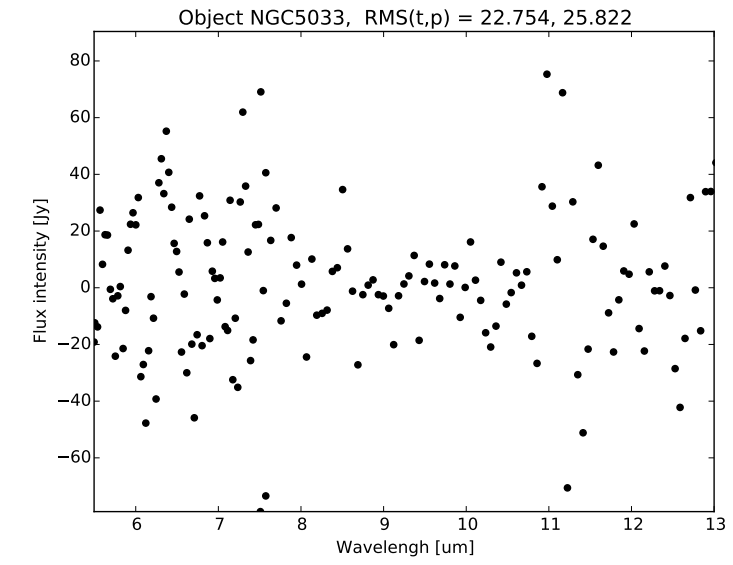

Figura B.54: Gráfico de RMS do objeto NGC5033 com os valores total e parcial. 


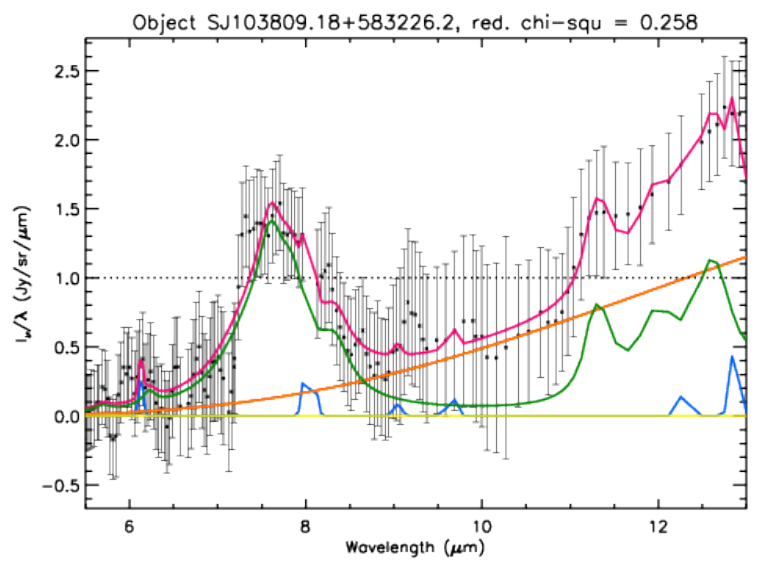

Figura B.55: Espectro do objeto SJ103809.18+583226.2 decomposto com o PAHFIT e o valor do $\chi^{2}$ reduzido.

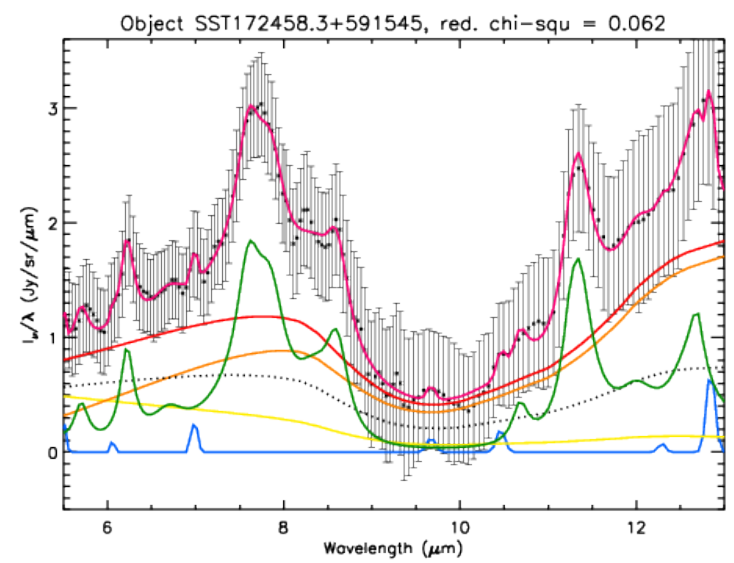

Figura B.57: Espectro do objeto SST172458.3+591545 decomposto com o PAHFIT e o valor do $\chi^{2}$ reduzido.

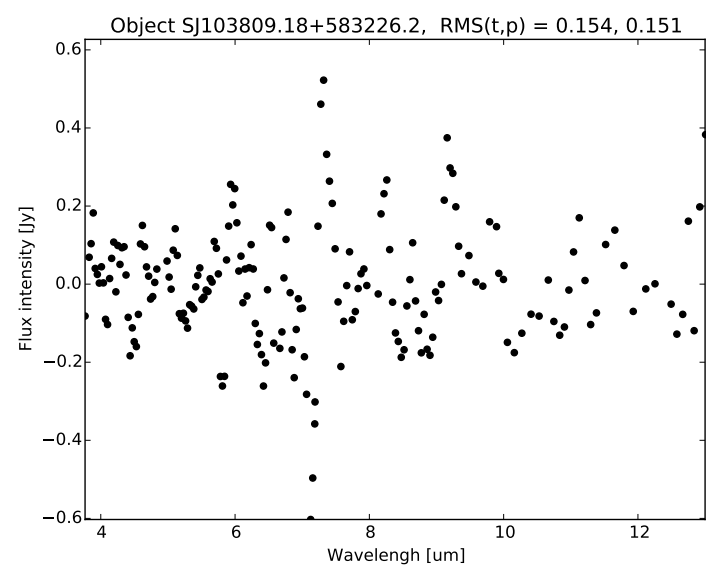

Figura B.56: Gráfico de RMS do objeto SJ103809.18+583226.2 com os valores total e parcial.

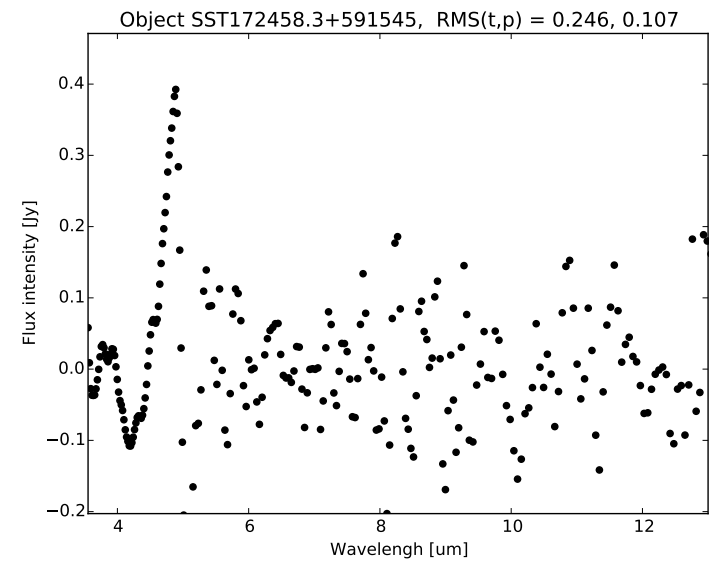

Figura B.58: Gráfico de RMS do objeto SST172458.3+591545 com os valores total e parcial. 


\section{Apêndice $\mathrm{C}$}

\section{Perfis da banda de $6.2 \mu m$}

C.1 Melhor ajuste com o pah62_curvefit.py

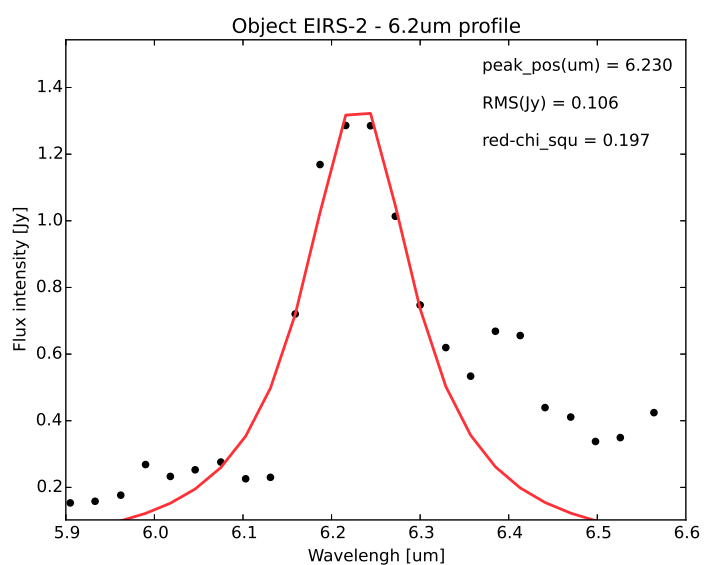

Figura C.1: Perfil da banda de $6.2 \mu m$ do objeto EIRS-2 ajustado com curve_fit e para $6.0 \leq \lambda \leq$ 6.34 .

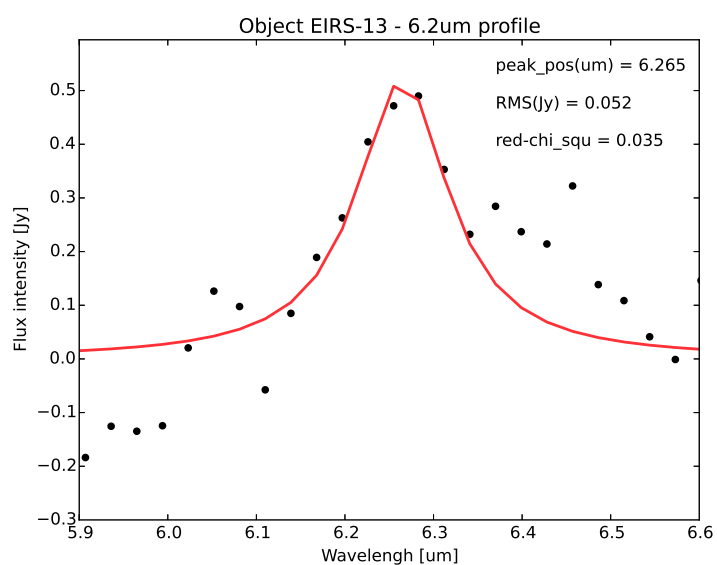

Figura C.2: Perfil da banda de $6.2 \mu \mathrm{m}$ do objeto EIRS-13 ajustado com curve_fit e para $6.10 \leq \lambda \leq$ 6.32 . 


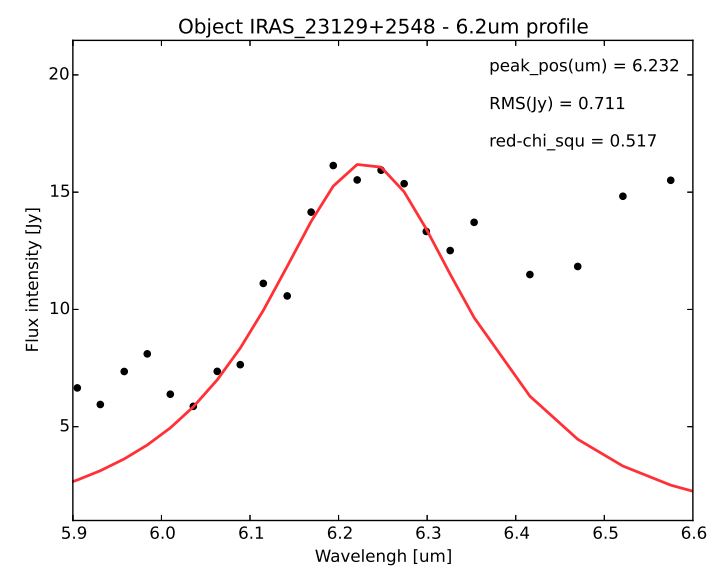

Figura C.3: Perfil da banda de $6.2 \mu m$ do objeto IRAS_23129+2548 ajustado com curve_fit e para $6.05 \leq \lambda \leq 6.30$.

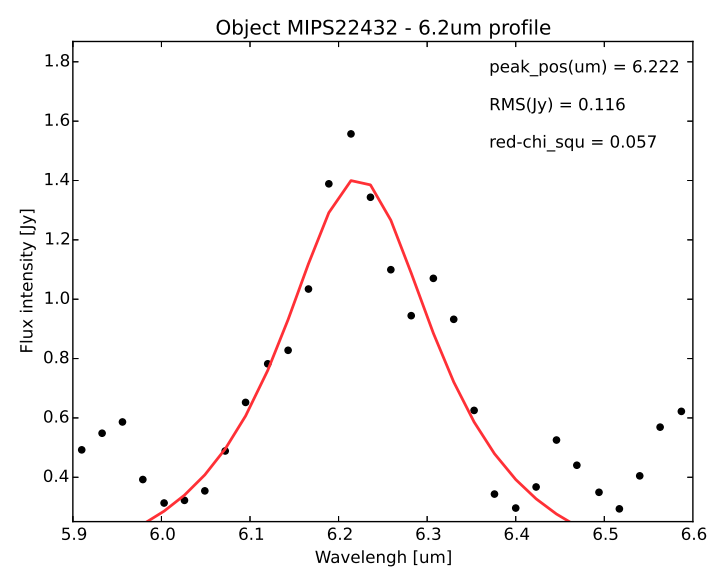

Figura C.5: Perfil da banda de $6.2 \mu \mathrm{m}$ do objeto MIPS22432 ajustado com curve_fit.

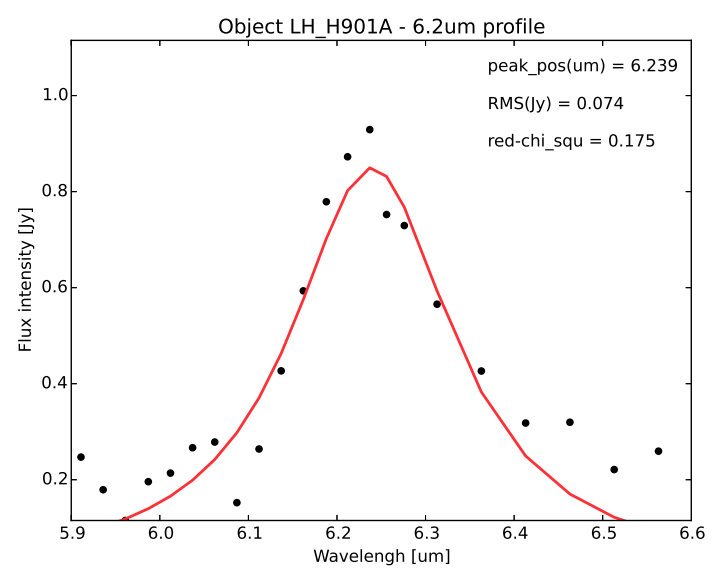

Figura C.4: Perfil da banda de $6.2 \mu m$ do objeto LH_H901A ajustado com curve_fit.

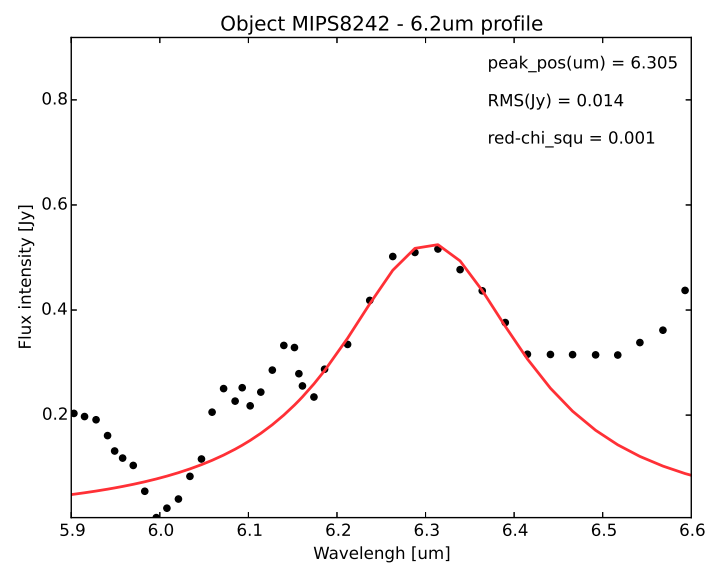

Figura C.6: Perfil da banda de $6.2 \mu m$ do objeto MIPS8242 ajustado com curve_fit e para $6.168 \leq$ $\lambda \leq 6.42$. 


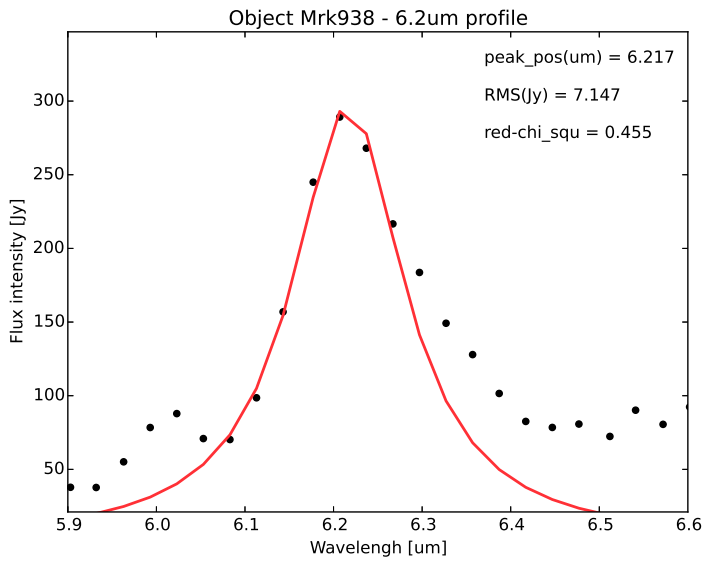

Figura C.7: Perfil da banda de $6.2 \mu \mathrm{m}$ do objeto Mrk938 ajustado com curve_fit e para $6.07 \leq$ $\lambda \leq 6.28$.

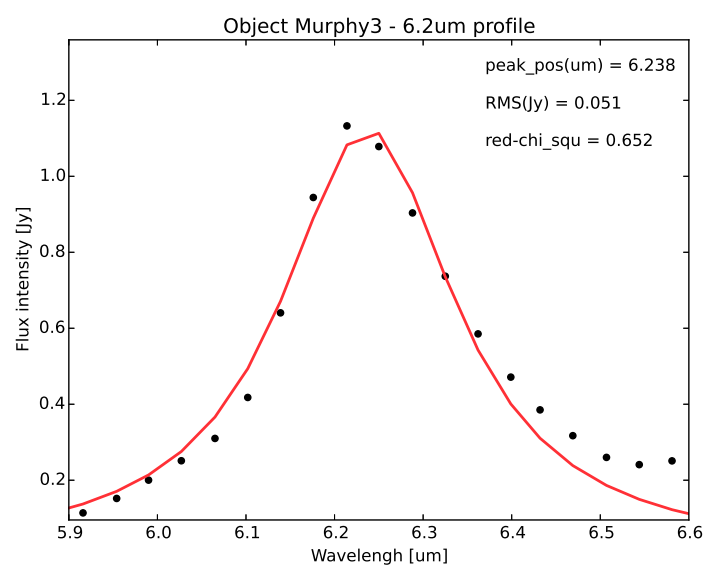

Figura C.9: Perfil da banda de $6.2 \mu m$ do objeto Murphy3 ajustado com curve_fit.

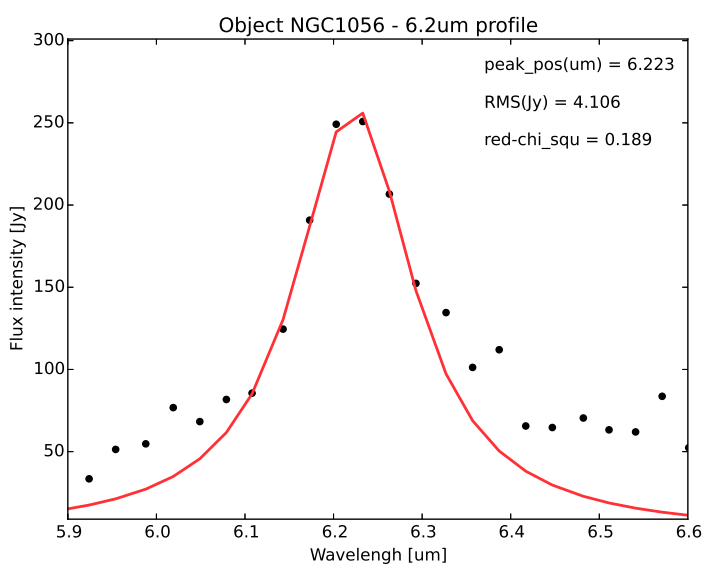

Figura C.11: Perfil da banda de $6.2 \mu \mathrm{m}$ do objeto NGC1056 ajustado com curve_fit e para $6.09 \leq \lambda \leq 6.30$.

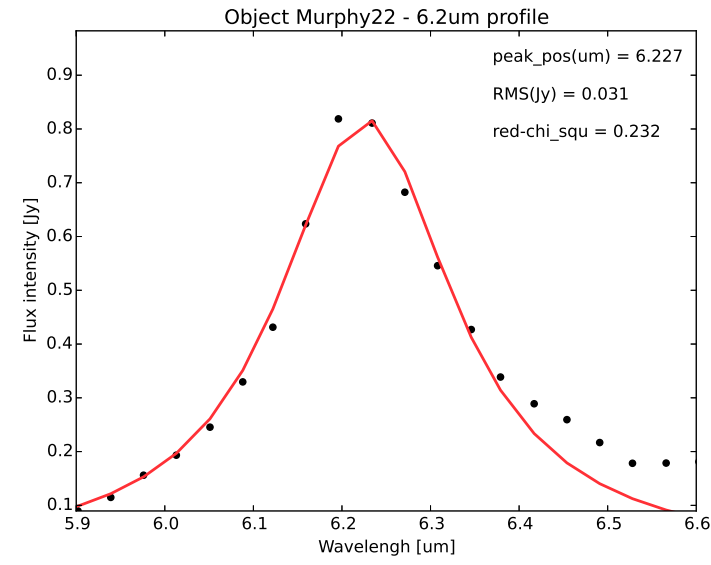

Figura C.8: Perfil da banda de $6.2 \mu m$ do objeto Murphy22 ajustado com curve_fit.

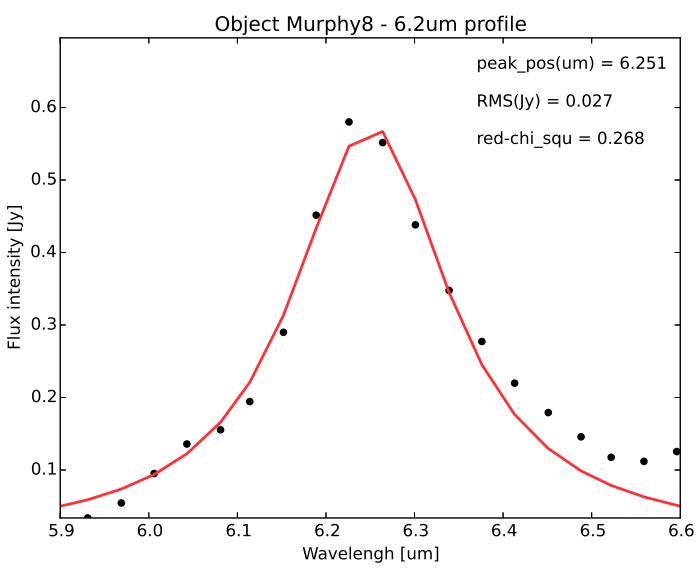

Figura C.10: Perfil da banda de $6.2 \mu \mathrm{m}$ do objeto Murphy8 ajustado com curve_fit.

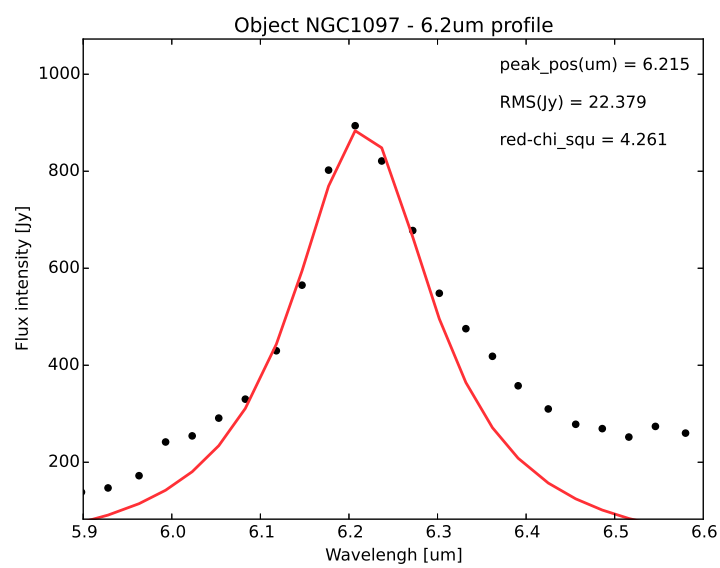

Figura C.12: Perfil da banda de $6.2 \mu m$ do objeto NGC1097 ajustado com curve_fit e para $6.08 \leq$ $\lambda \leq 6.30$. 


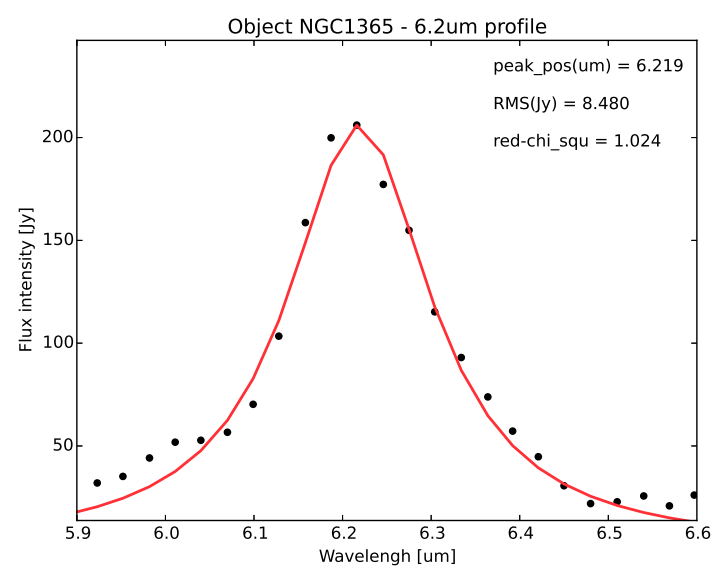

Figura C.13: Perfil da banda de $6.2 \mu m$ do objeto NGC1365 ajustado com curve_fit.

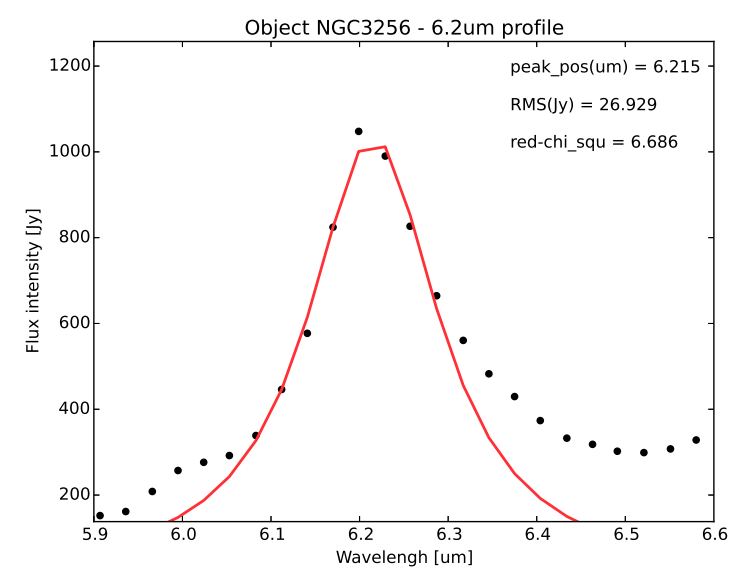

Figura C.15: Perfil da banda de $6.2 \mu m$ do objeto NGC3256 ajustado com curve_fit e para $6.07 \leq \lambda \leq 6.31$.

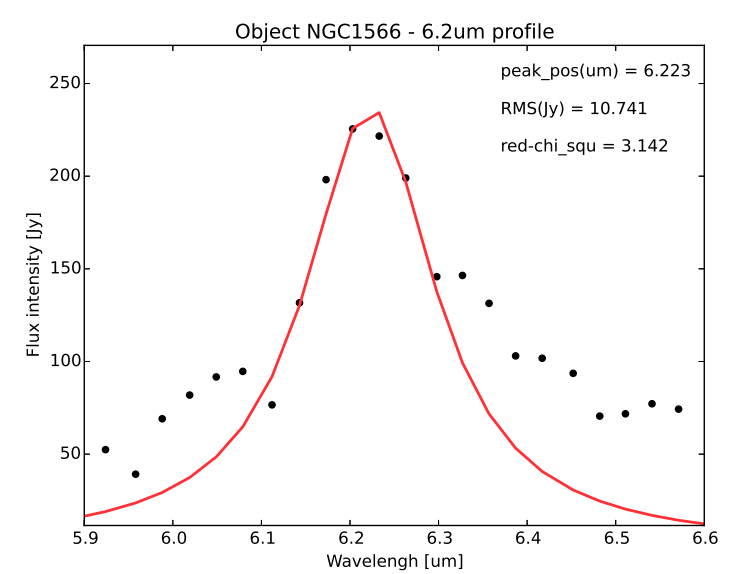

Figura C.14: Perfil da banda de $6.2 \mu m$ do objeto NGC1566 ajustado com curve_fit e para $6.10 \leq$ $\lambda \leq 6.31$.

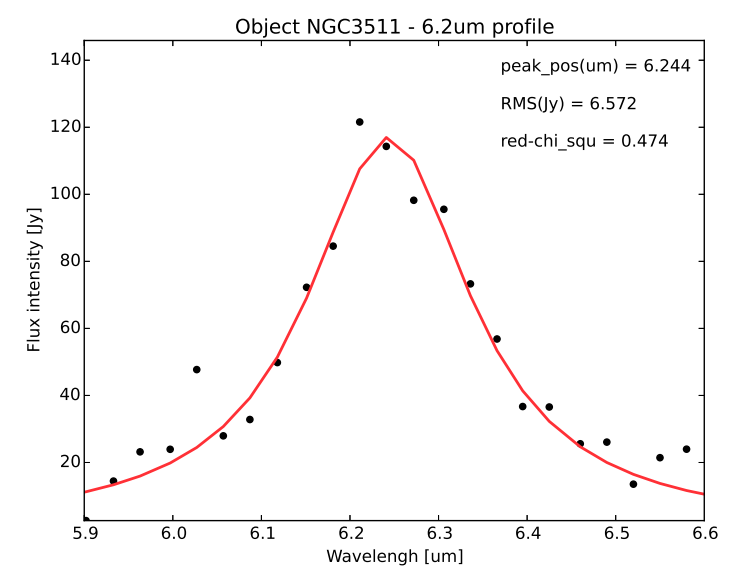

Figura C.16: Perfil da banda de $6.2 \mu m$ do objeto NGC3511 ajustado com curve_fit. 


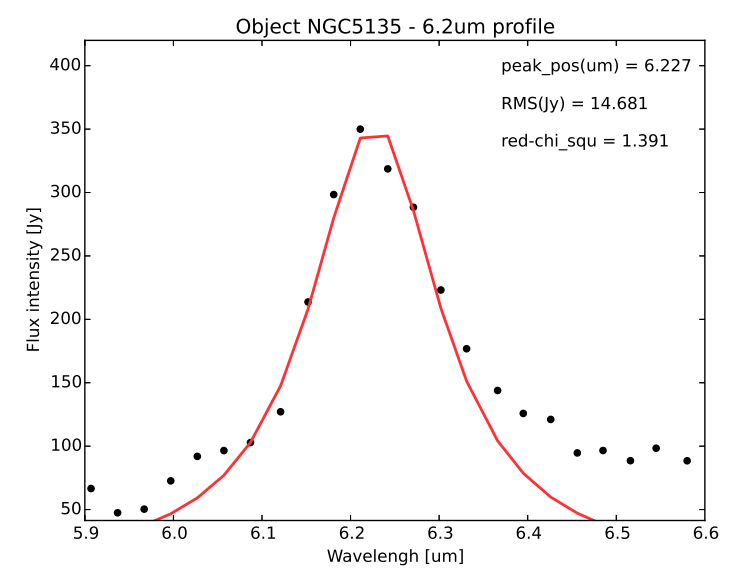

Figura C.17: Perfil da banda de $6.2 \mu m$ do objeto NGC5135 ajustado com curve_fit e para $6.07 \leq \lambda \leq 6.33$.

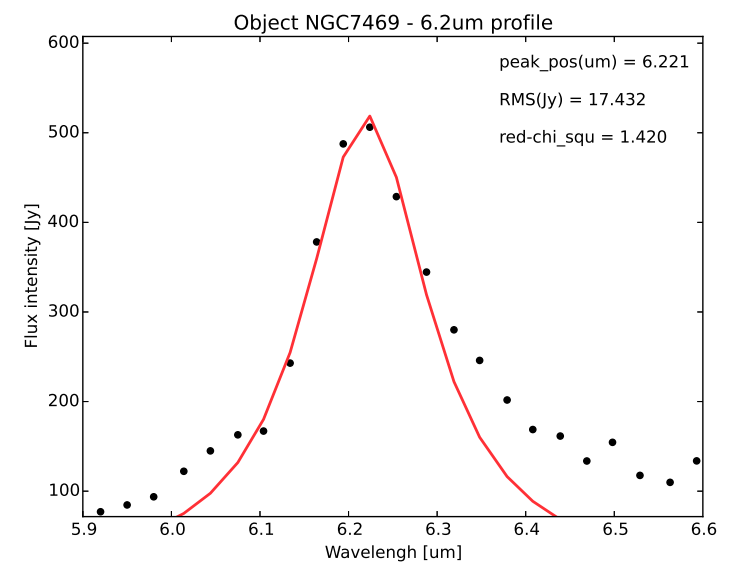

Figura C.19: Perfil da banda de $6.2 \mu \mathrm{m}$ do objeto NGC7469 ajustado com curve_fit e para $6.1 \leq \lambda \leq 6.3$.

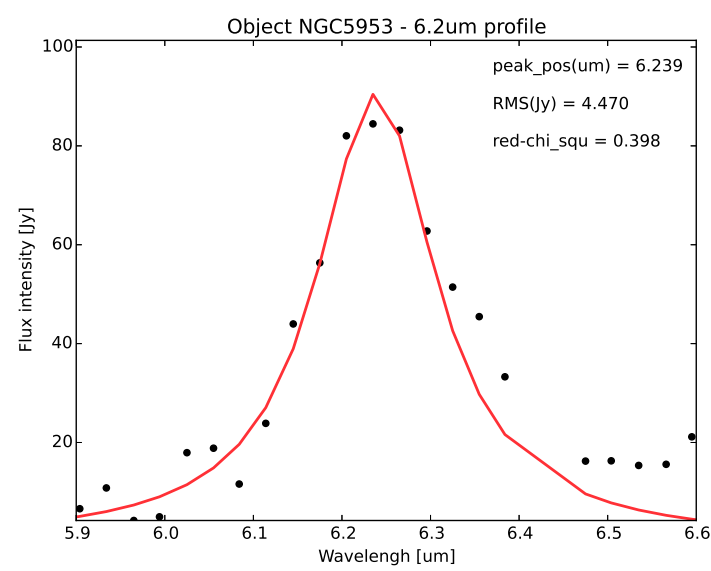

Figura C.18: Perfil da banda de $6.2 \mu m$ do objeto NGC5953 ajustado com curve_fit e para $6.05 \leq$ $\lambda \leq 6.32$.

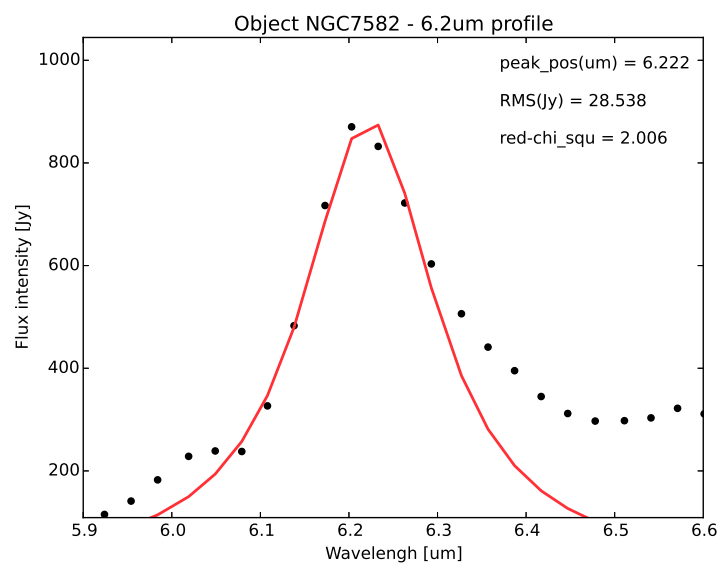

Figura C.20: Perfil da banda de $6.2 \mu m$ do objeto NGC7582 ajustado com curve_fit e para $6.06 \leq$ $\lambda \leq 6.30$. 


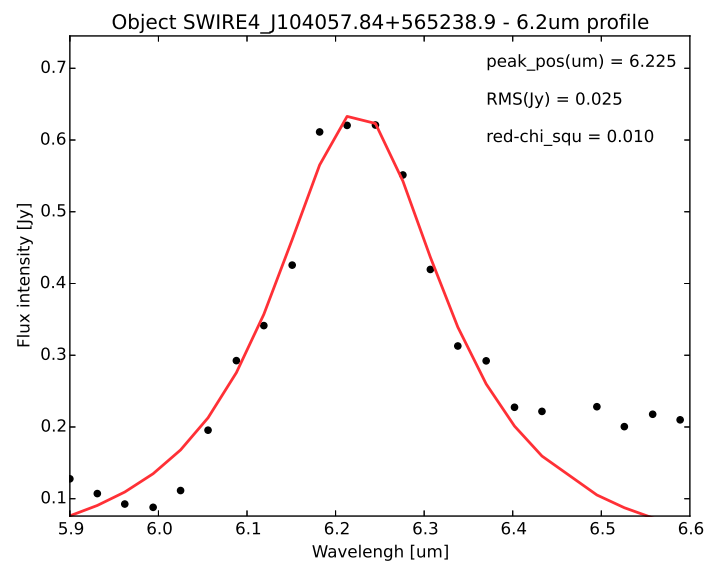

Figura C.21: Perfil da banda de $6.2 \mu \mathrm{m}$ do objeto SWIRE4_J104057.84+565238.9 ajustado com curve_fit.

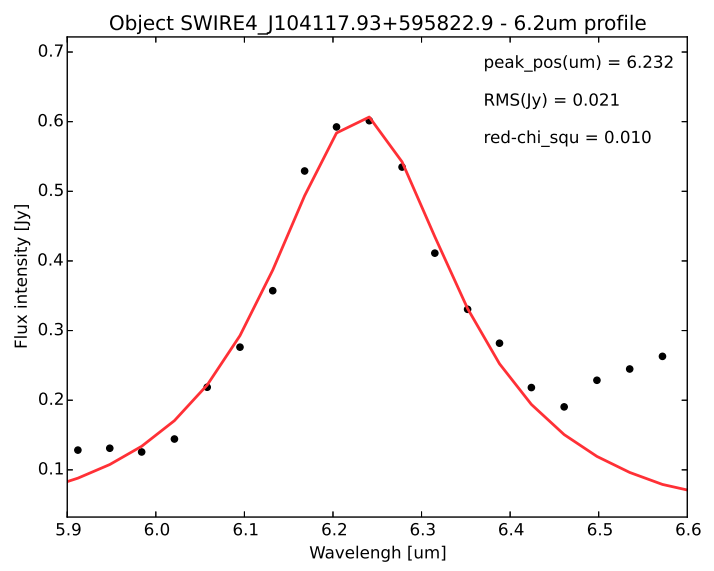

Figura C.22: Perfil da banda de $6.2 \mu m$ do objeto SWIRE4_J104117.93+595822.9 ajustado com curve_fit. 


\section{C.2 Melhor ajuste com o pah62_opt2.py}

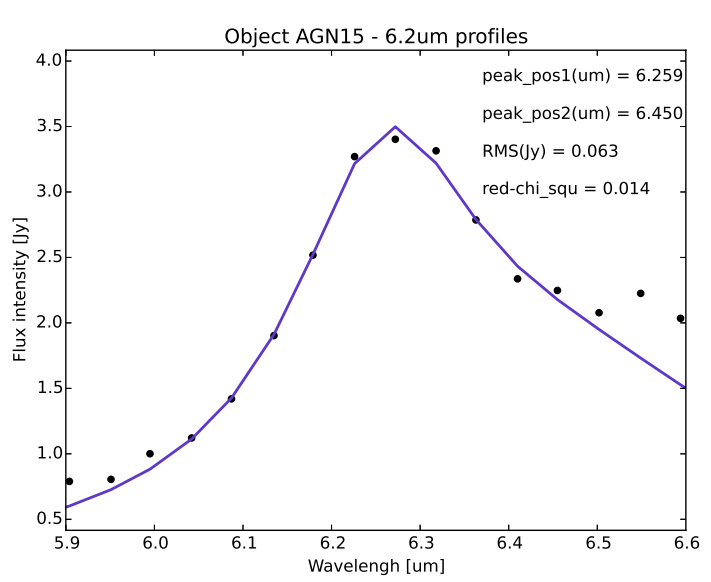

Figura C.23: Perfil da banda de $6.2 \mu \mathrm{m}$ do objeto AGN15 ajustado com minimize.

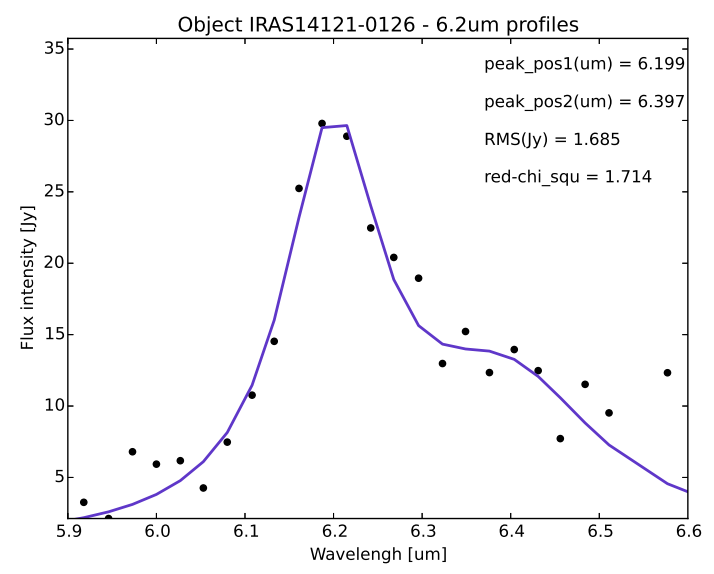

Figura C.25: Perfil da banda de $6.2 \mu \mathrm{m}$ do objeto IRAS14121-0126 ajustado com minimize.

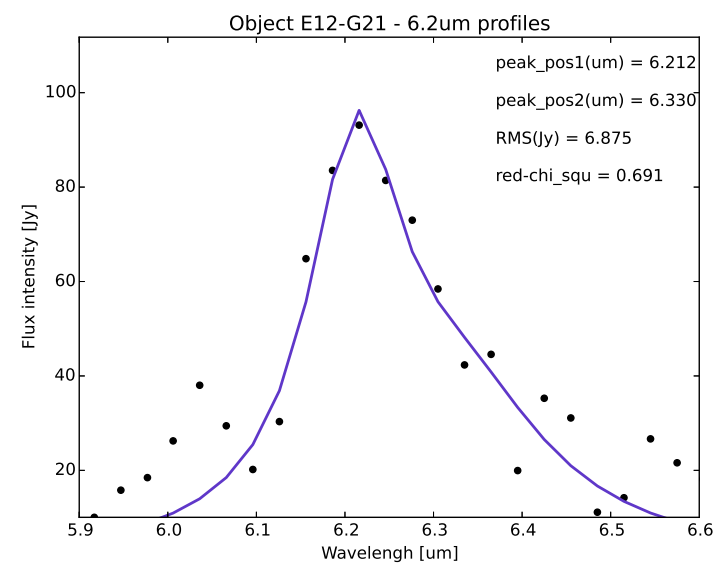

Figura C.24: Perfil da banda de $6.2 \mu m$ do objeto E12-G21 ajustado com minimize.

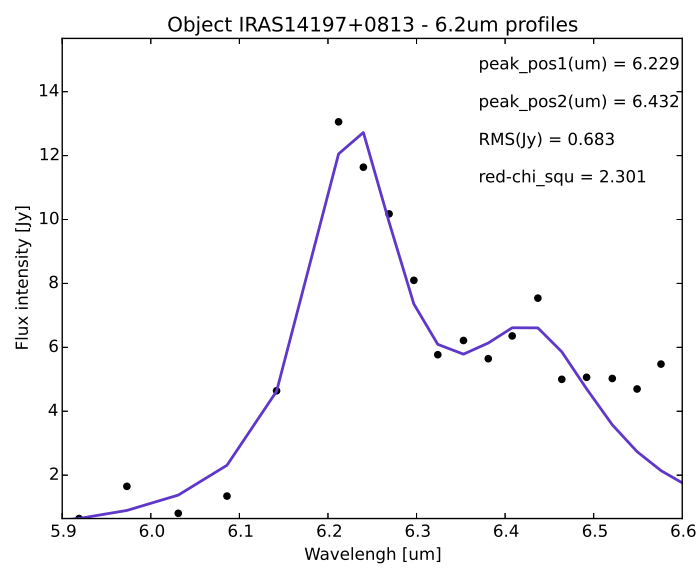

Figura C.26: Perfil da banda de $6.2 \mu m$ do objeto IRAS14197+0813 ajustado com minimize. 


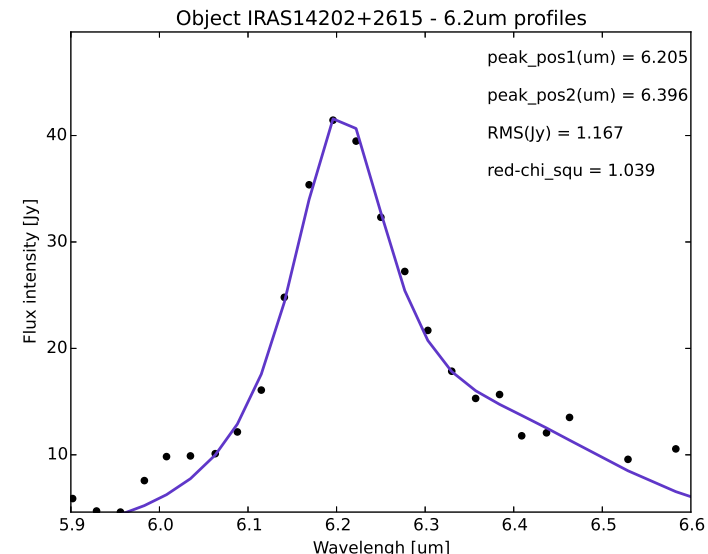

Figura C.27: Perfil da banda de $6.2 \mu \mathrm{m}$ do objeto IRAS14202+2615 ajustado com minimize.

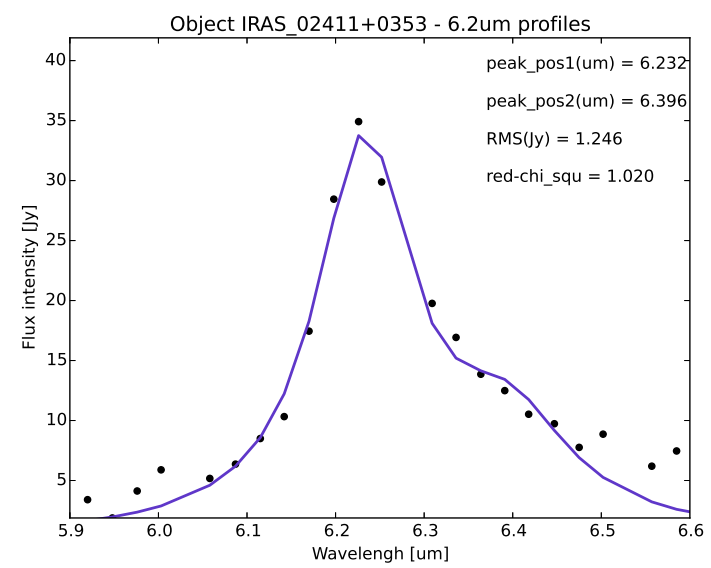

Figura C.29: Perfil da banda de $6.2 \mu m$ do objeto IRAS_02411+0353 ajustado com minimize.

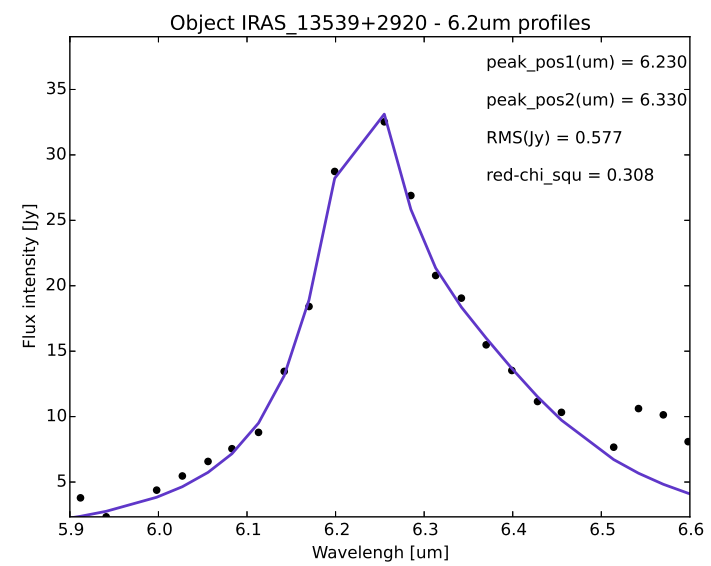

Figura C.31: Perfil da banda de $6.2 \mu m$ do objeto IRAS_13539+2920 ajustado com minimize.

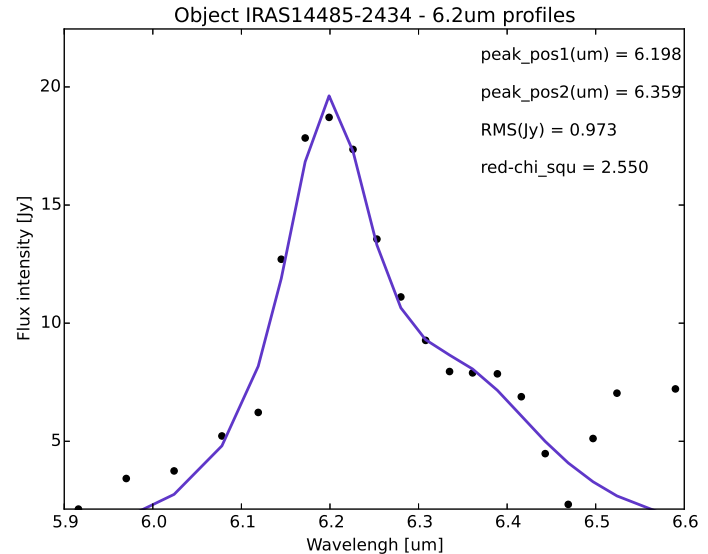

Figura C.28: Perfil da banda de $6.2 \mu m$ do objeto IRAS14485-2434 ajustado com minimize.

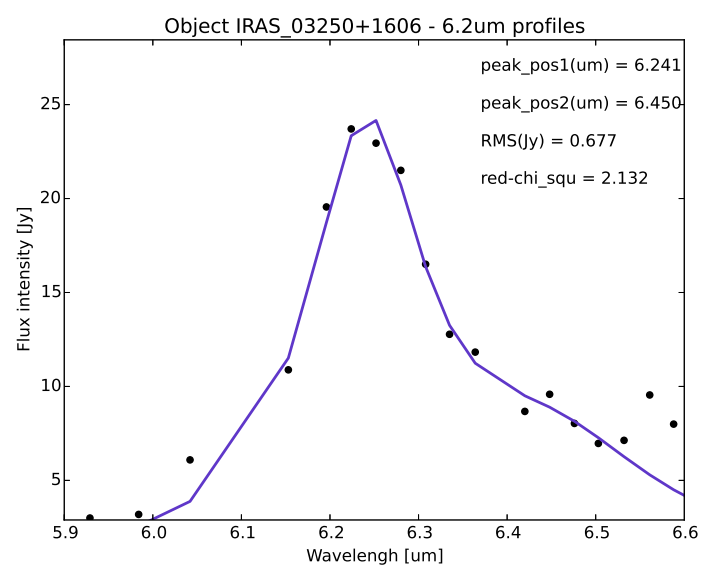

Figura C.30: Perfil da banda de $6.2 \mu \mathrm{m}$ do objeto IRAS_03250+1606 ajustado com minimize.

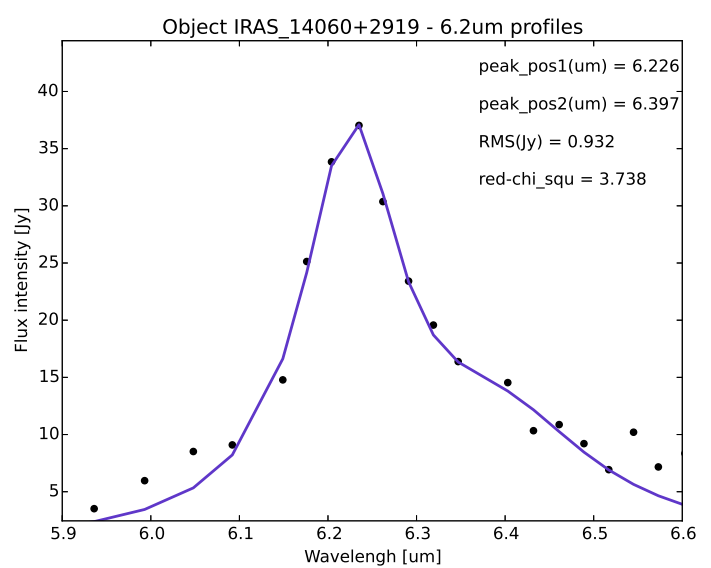

Figura C.32: Perfil da banda de $6.2 \mu m$ do objeto IRAS_14060+2919 ajustado com minimize. 


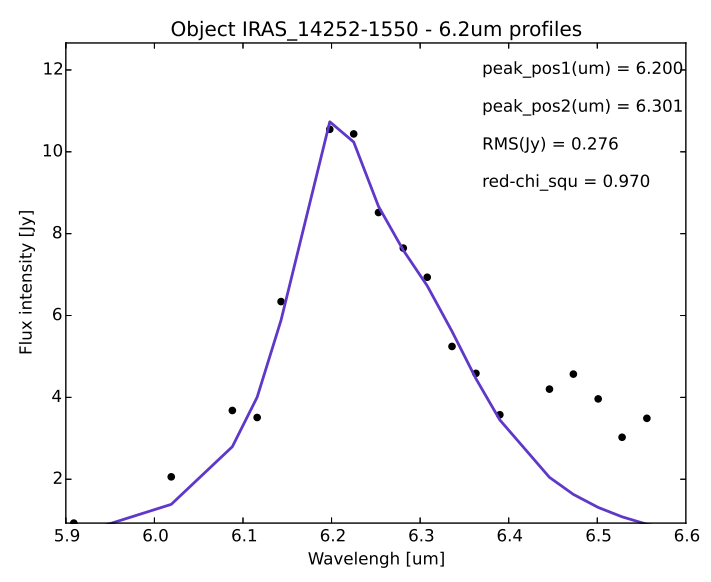

Figura C.33: Perfil da banda de $6.2 \mu \mathrm{m}$ do objeto IRAS_14252-1550 ajustado com minimize e para $6.1 \leq \lambda \leq 6.4,6.10 \leq \lambda_{i 1} \leq 6.26$, None $\leq \lambda_{i 2} \leq 6.4, \lambda_{i 1}=6.18, \lambda_{i 2}=6.28 \mathrm{e}$ $\gamma_{i 2}=0.20$.

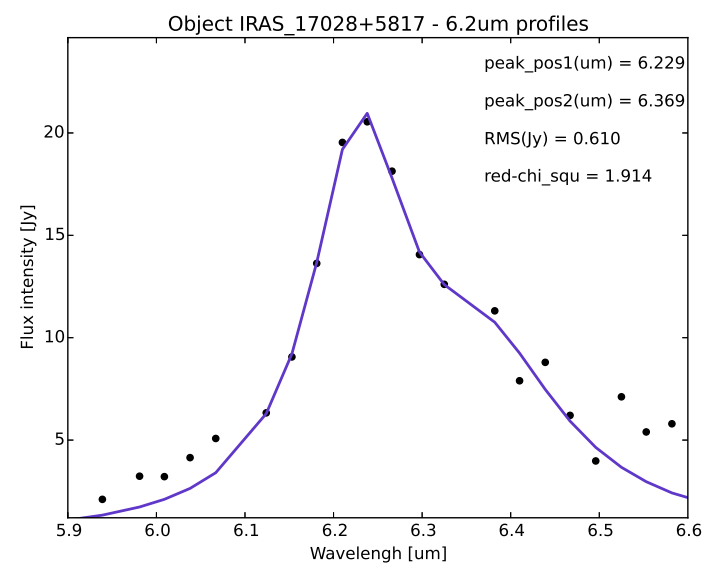

Figura C.35: Perfil da banda de $6.2 \mu \mathrm{m}$ do objeto IRAS_17028+5817 ajustado com minimize.

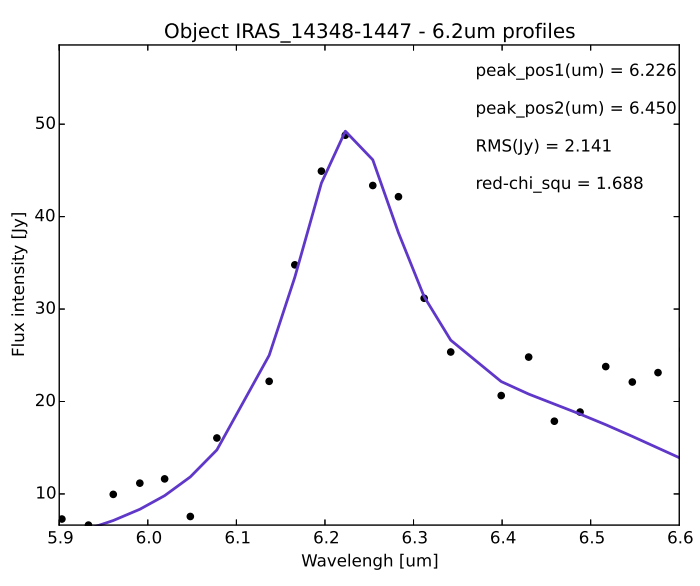

Figura C.34: Perfil da banda de $6.2 \mu m$ do objeto IRAS_14348-1447 ajustado com minimize.

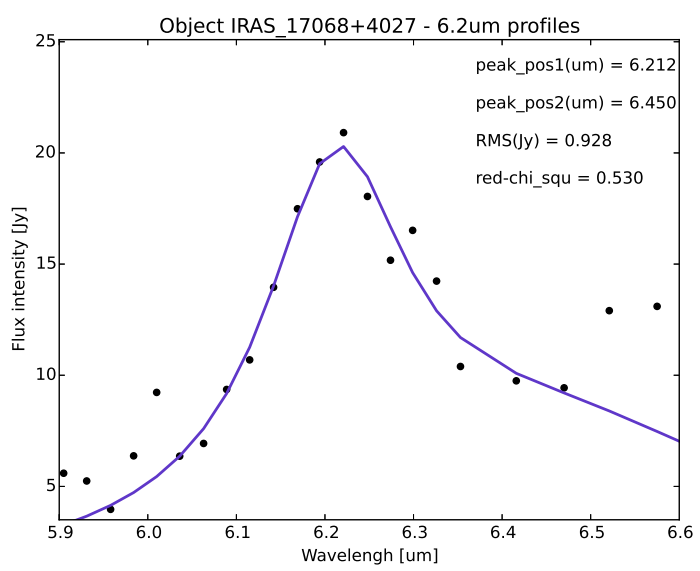

Figura C.36: Perfil da banda de $6.2 \mu m$ do objeto IRAS_17068+4027 ajustado com minimize. 


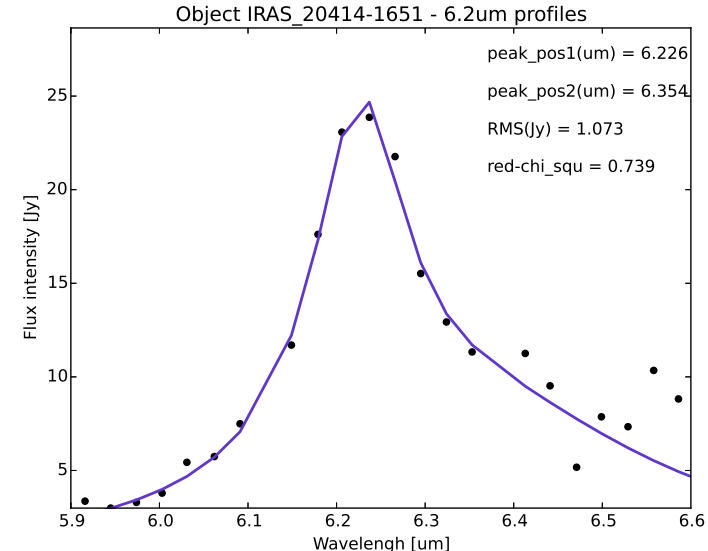

Figura C.37: Perfil da banda de $6.2 \mu m$ do objeto IRAS_20414-1651 ajustado com minimize.

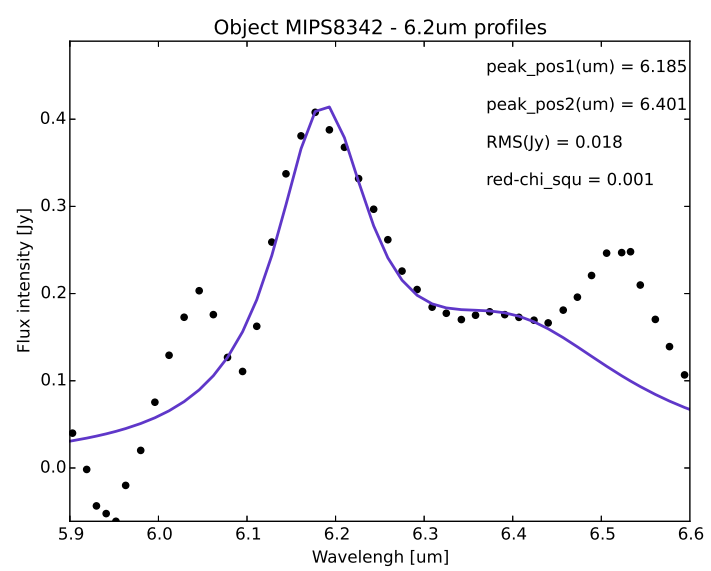

Figura C.39: Perfil da banda de $6.2 \mu m$ do objeto MIPS8342 ajustado com minimize e para $6.08 \leq \lambda \leq 6.44,6.10 \leq \lambda_{i 1} \leq 6.25$ e $6.33 \leq$ $\lambda_{i 2} \leq 6.42$.

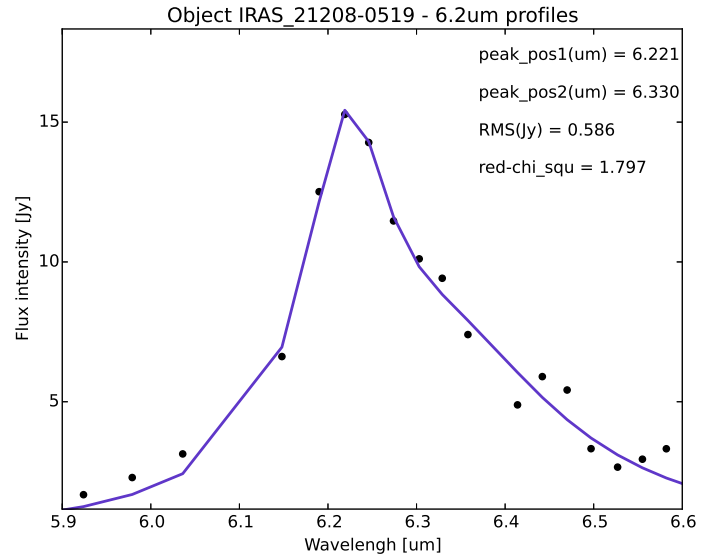

Figura C.38: Perfil da banda de $6.2 \mu m$ do objeto IRAS_21208-0519 ajustado com minimize.

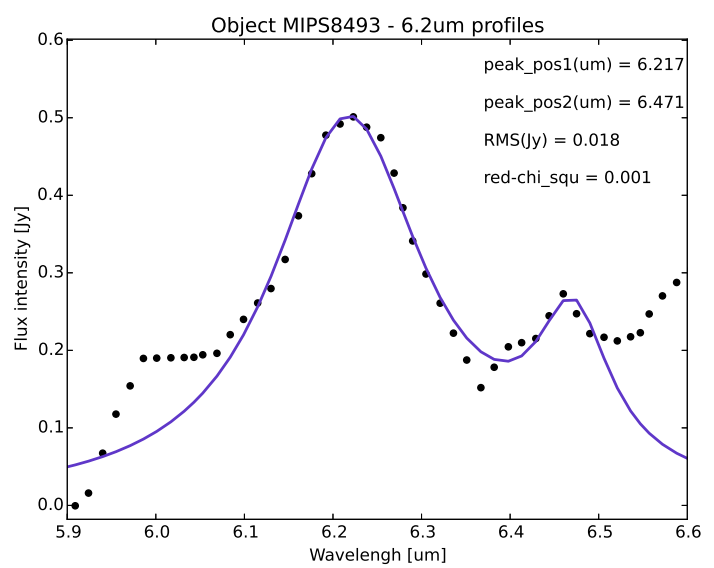

Figura C.40: Perfil da banda de $6.2 \mu \mathrm{m}$ do objeto MIPS8493 ajustado com minimize e para $6.06 \leq$ $\lambda \leq 6.51,6.10 \leq \lambda_{i 1} \leq 6.25,6.36 \leq \lambda_{i 2} \leq 6.48 \mathrm{e}$ $\lambda_{i 2}=6.45$. 


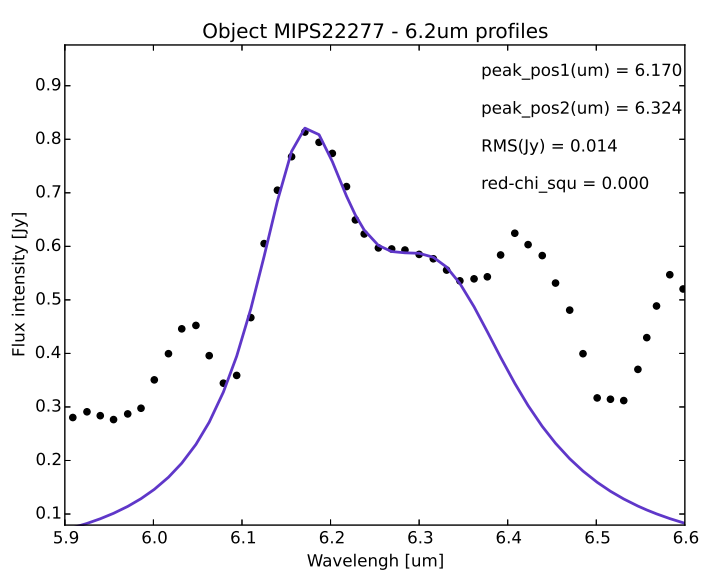

Figura C.41: Perfil da banda de $6.2 \mu m$ do objeto MIPS22277 ajustado com minimize e para $6.10 \leq \lambda_{i 1} \leq 6.24,6.241 \leq \lambda_{i 2} \leq 6.34$, $\lambda_{i 1}=6.18$ e $\lambda_{i 2}=6.29$.

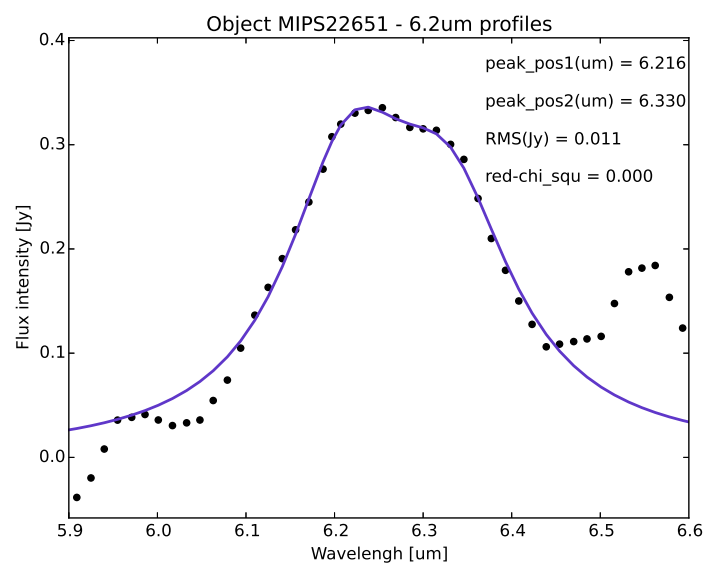

Figura C.43: Perfil da banda de $6.2 \mu m$ do objeto MIPS22651 ajustado com minimize.

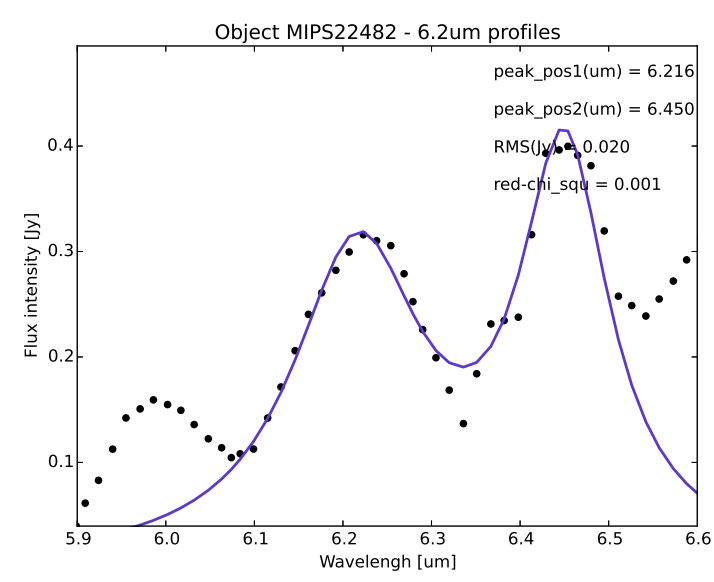

Figura C.42: Perfil da banda de $6.2 \mu m$ do objeto MIPS22482 ajustado com minimize.

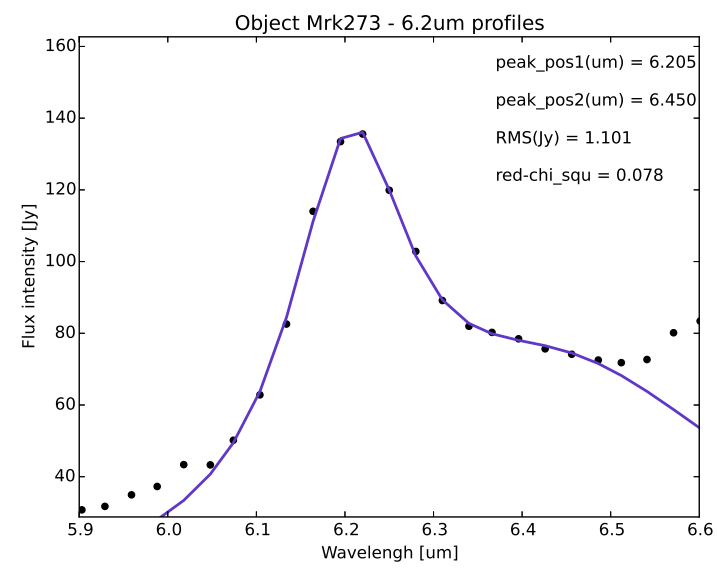

Figura C.44: Perfil da banda de $6.2 \mu m$ do objeto Mrk273 ajustado com minimize. 


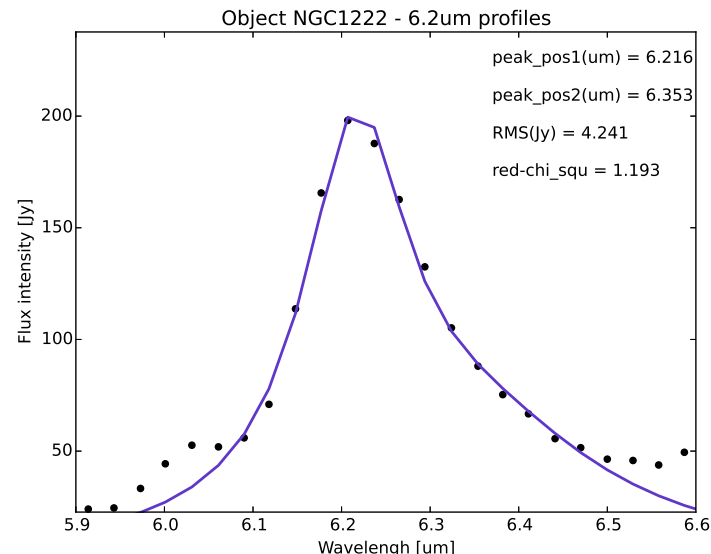

Figura C.45: Perfil da banda de $6.2 \mu m$ do objeto NGC1222 ajustado com minimize.

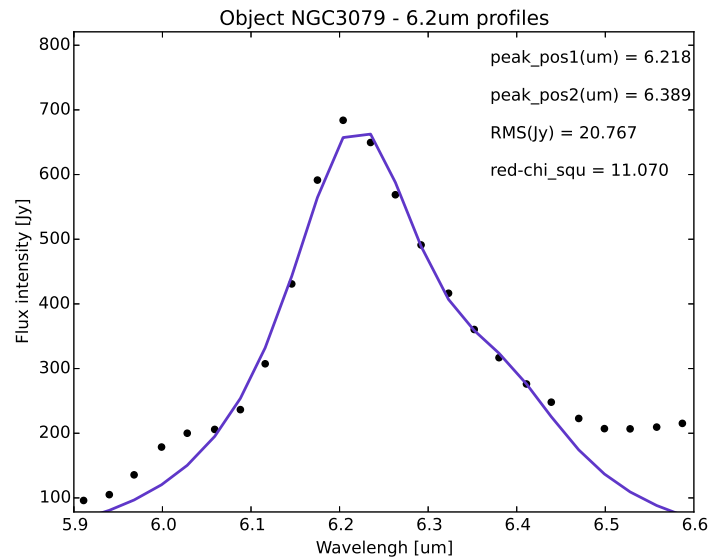

Figura C.47: Perfil da banda de $6.2 \mu \mathrm{m}$ do objeto NGC3079 ajustado com minimize e para $6.07 \leq \lambda \leq 6.49,6.10 \leq \lambda_{i 1} \leq 6.235,6.22 \leq$ $\lambda_{i 2} \leq 6.40$ e $\lambda_{i 1}=6.20$.

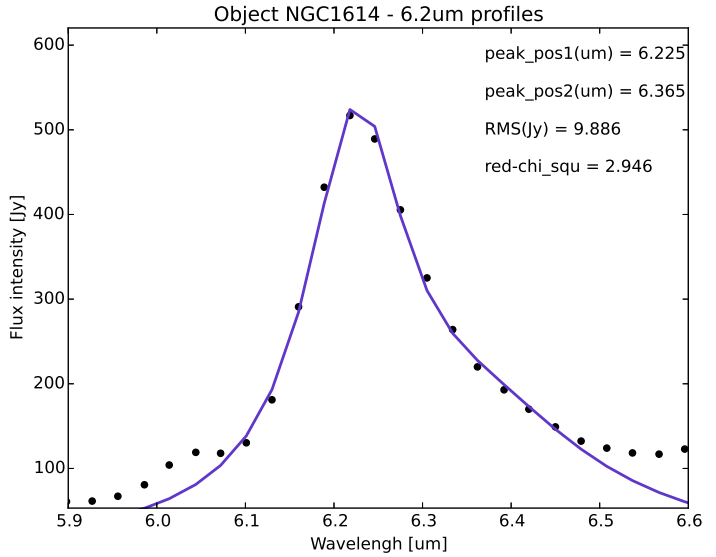

Figura C.46: Perfil da banda de $6.2 \mu \mathrm{m}$ do objeto NGC1614 ajustado com minimize e para $6.08 \leq$ $\lambda \leq 6.50,6.10 \leq \lambda_{i 1} \leq 6.24,6.24 \leq \lambda_{i 2} \leq 6.40$, e $\lambda_{i 2}=6.26$.

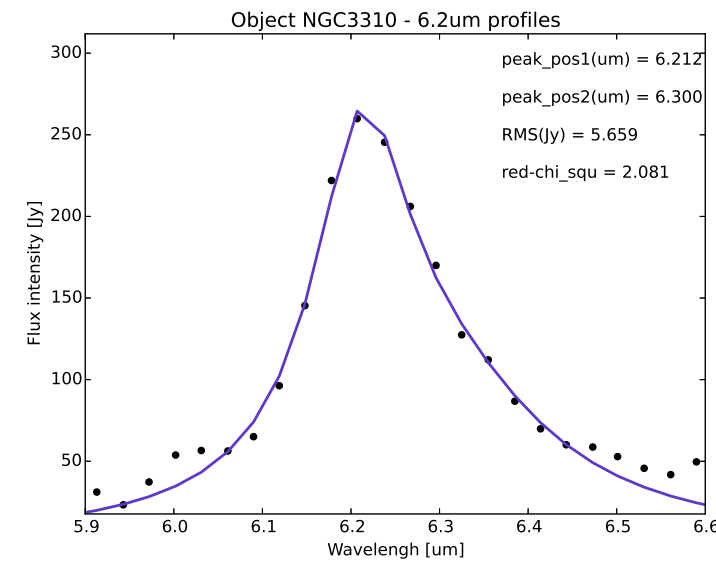

Figura C.48: Perfil da banda de $6.2 \mu m$ do objeto NGC3310 ajustado com minimize e para $6.10 \leq$ $\lambda \leq 6.49,6.10 \leq \lambda_{i 1} \leq 6.23,6.3 \leq \lambda_{i 2} \leq 6.47$, $\lambda_{i 1}=6.21, \lambda_{i 2}=6.35$ e $\gamma_{i 2}=0.30$. 


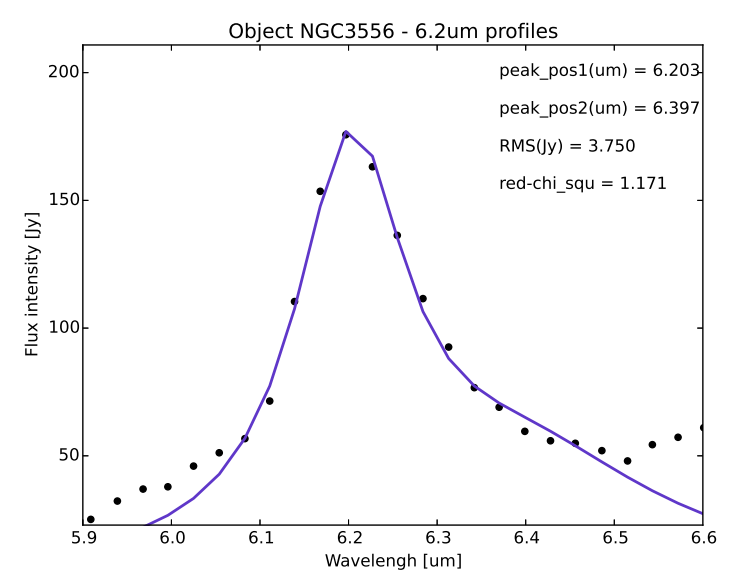

Figura C.49: Perfil da banda de $6.2 \mu \mathrm{m}$ do objeto NGC3556 ajustado com minimize.

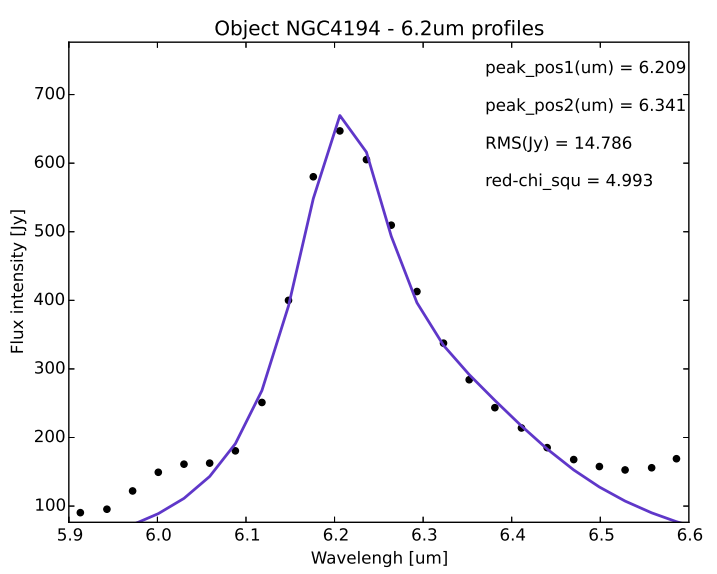

Figura C.51: Perfil da banda de $6.2 \mu m$ do objeto NGC4194 ajustado com minimize e para $6.07 \leq \lambda \leq 6.49,6.10 \leq \lambda_{i 1} \leq 6.23,6.25 \leq$ $\lambda_{i 2} \leq$ None, $\lambda_{i 1}=6.20$ e $\gamma_{i 2}=0.20$.

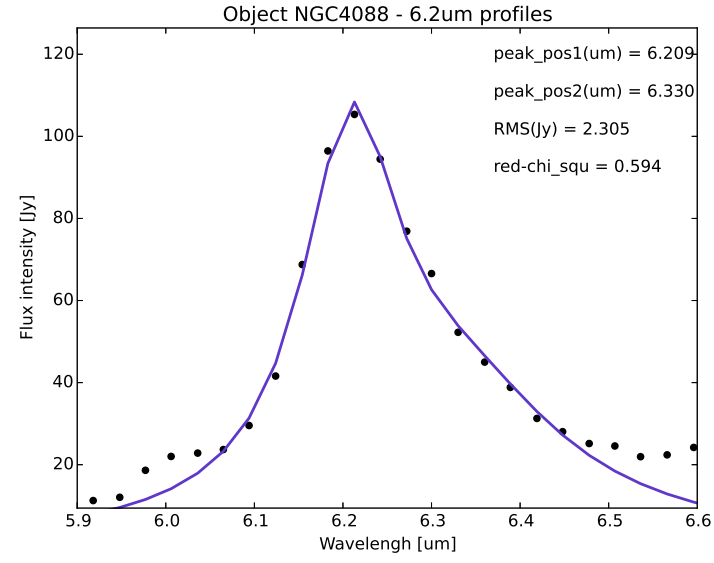

Figura C.50: Perfil da banda de $6.2 \mu m$ do objeto NGC4088 ajustado com minimize.

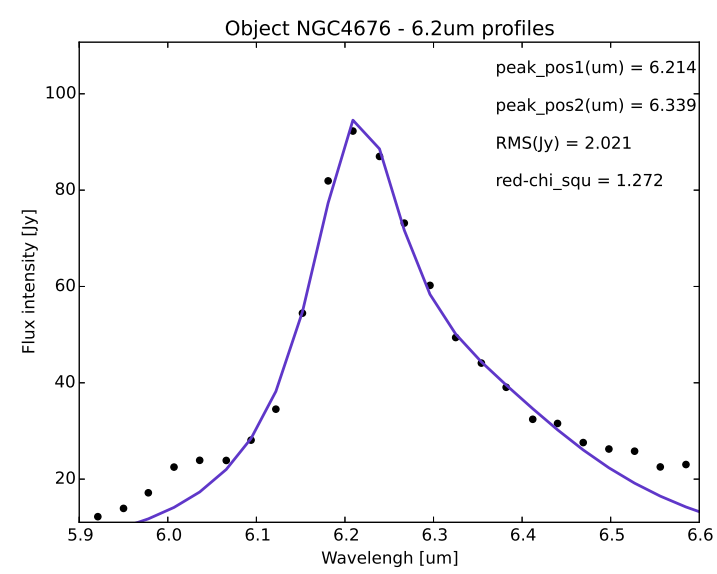

Figura C.52: Perfil da banda de $6.2 \mu \mathrm{m}$ do objeto NGC4676 ajustado com minimize e para $6041 \leq$ $\lambda \leq 6.49,6.10 \leq \lambda_{i 1} \leq 6.23,6.25 \leq \lambda_{i 2} \leq$ None, $\lambda_{i 1}=6.20$ e $\gamma_{i 2}=0.20$. 


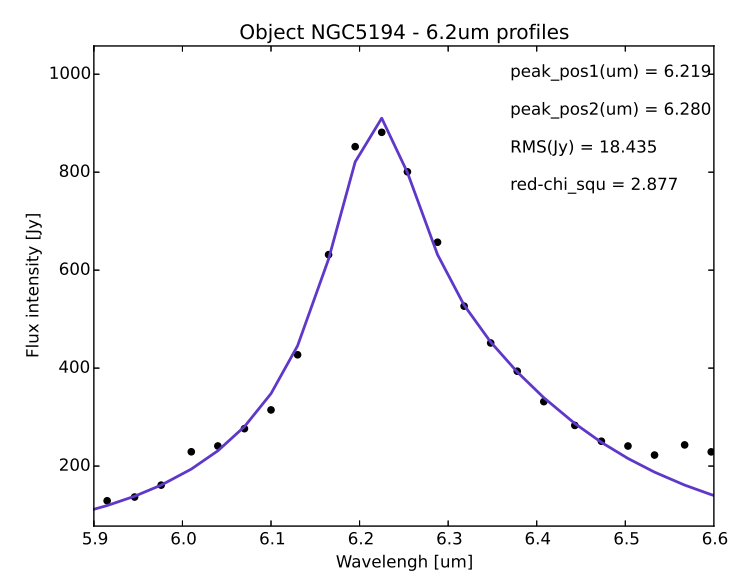

Figura C.53: Perfil da banda de $6.2 \mu \mathrm{m}$ do objeto NGC5194 ajustado com minimize e para $6.0 \leq \lambda \leq 6.49,6.09 \leq \lambda_{i 1} \leq 6.226$, None $\leq$ $\lambda_{i 2} \leq$ None e $\gamma_{i 2}=0.20$.

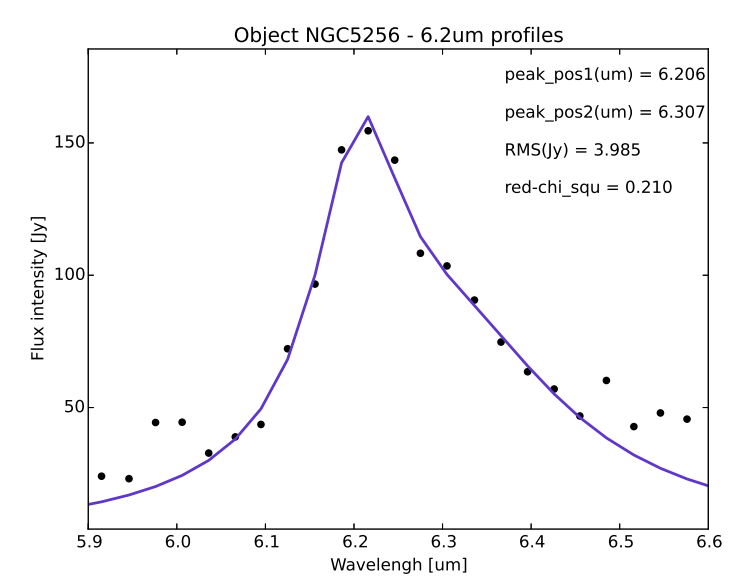

Figura C.54: Perfil da banda de $6.2 \mu m$ do objeto NGC5256 ajustado com minimize e para $6.02 \leq$ $\lambda \leq 6.46,6.09 \leq \lambda_{i 1} \leq 6.30$, None $\leq \lambda_{i 2} \leq$ None e $\gamma_{i 2}=0.20$. 


\section{C.3 Ajustes inconclusivos}

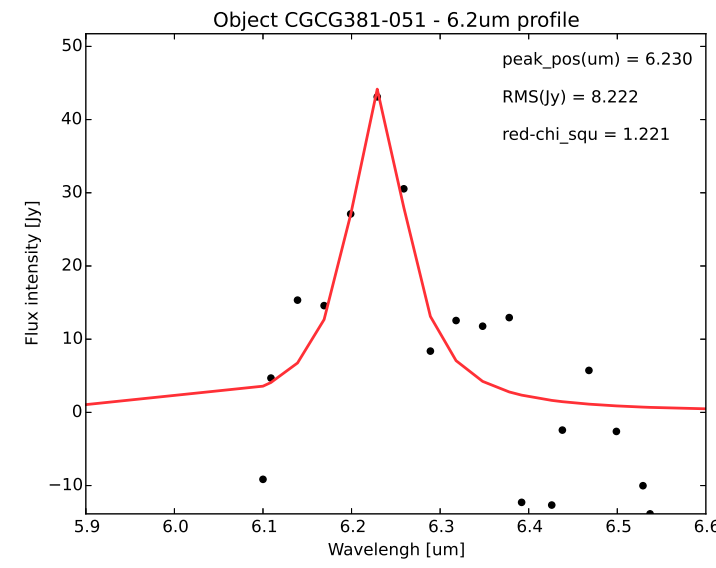

Figura C.55: Perfil da banda de $6.2 \mu \mathrm{m}$ do objeto CGCG381-051 ajustado com curve_fit.

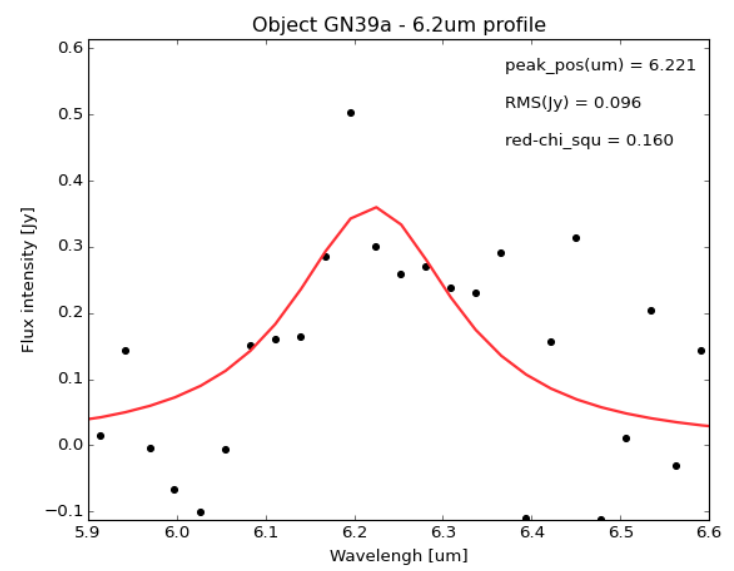

Figura C.57: Perfil da banda de $6.2 \mu \mathrm{m}$ do objeto GN39a ajustado com curve_fit.

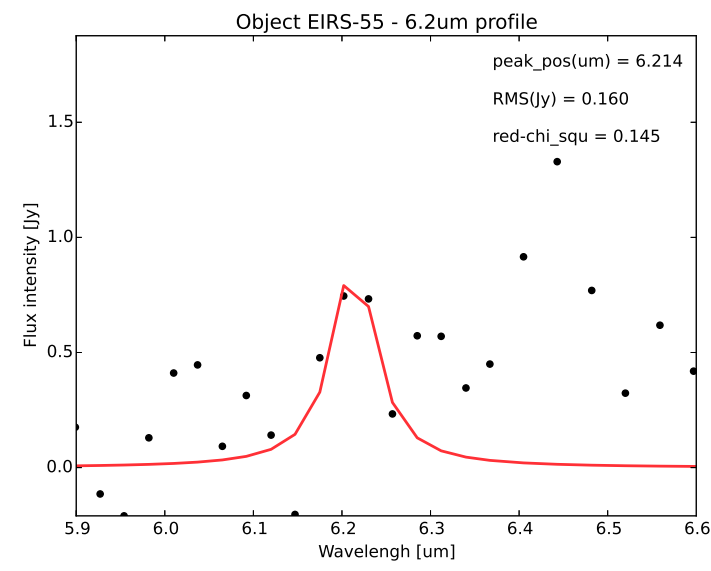

Figura C.56: Perfil da banda de $6.2 \mu m$ do objeto EIRS-55 ajustado com curve_fit e para $6.10 \leq \lambda \leq$ 6.28 .

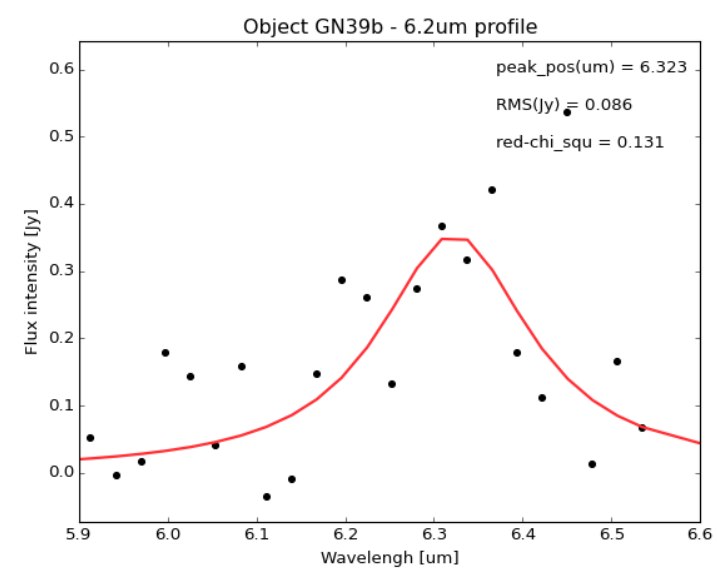

Figura C.58: Perfil da banda de $6.2 \mu m$ do objeto GN39b ajustado com curve_fit. 


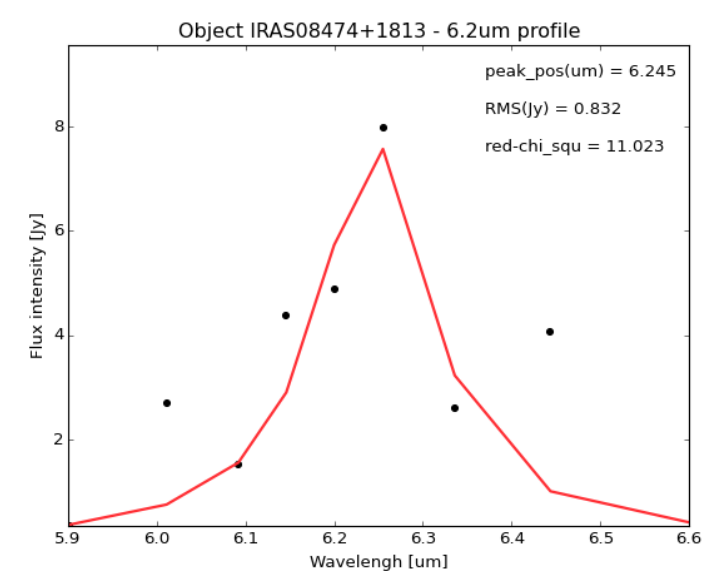

Figura C.59: Perfil da banda de $6.2 \mu m$ do objeto IRAS08474+1813 ajustado com curve_fit.

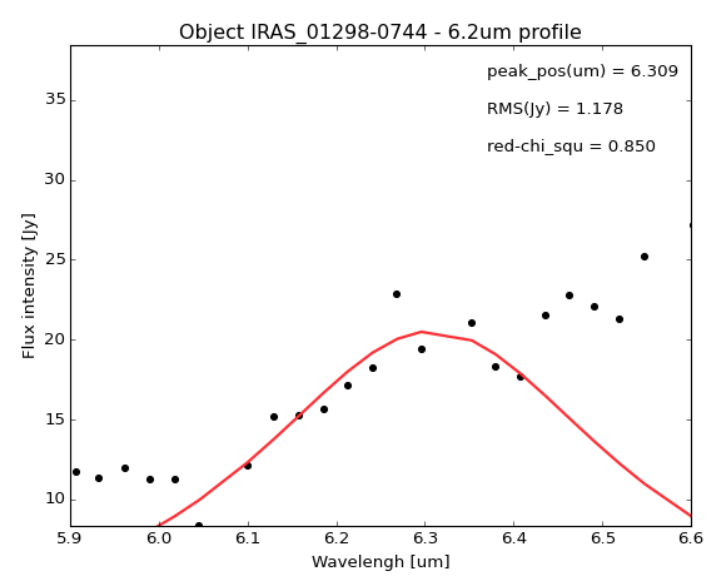

Figura C.61: Perfil da banda de $6.2 \mu m$ do objeto IRAS_01298-0744 ajustado com curve_fit.

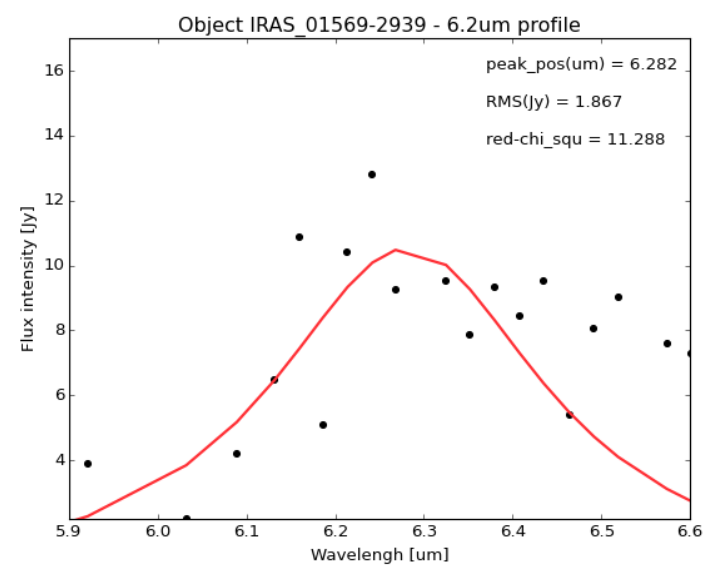

Figura C.63: Perfil da banda de $6.2 \mu m$ do objeto IRAS_01569-2939 ajustado com curve_fit.

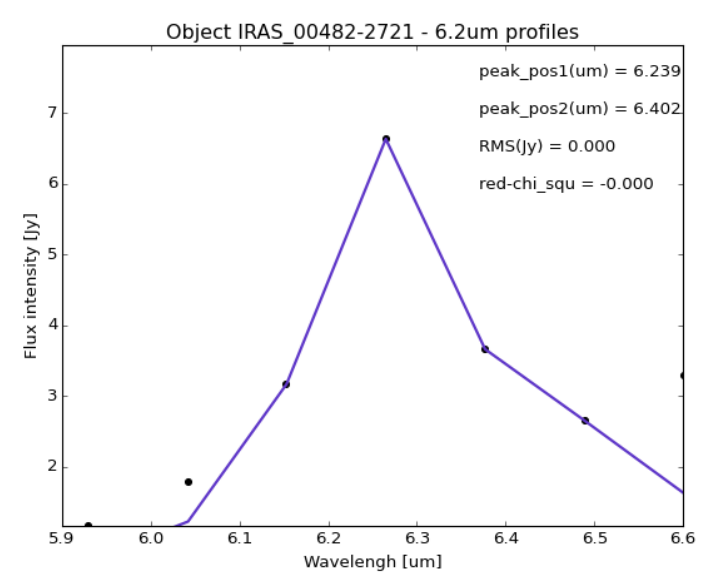

Figura C.60: Perfil da banda de $6.2 \mu m$ do objeto IRAS_00482-2721 ajustado com minimize.

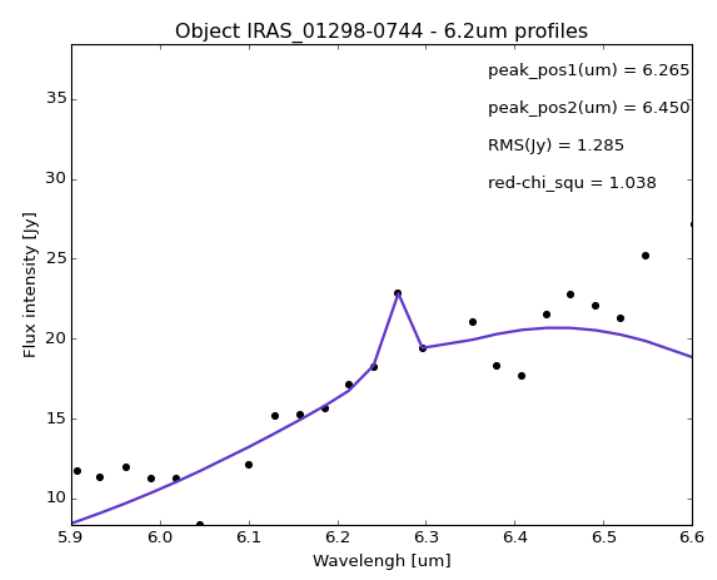

Figura C.62: Perfil da banda de $6.2 \mu m$ do objeto IRAS_01298-0744 ajustado com minimize.

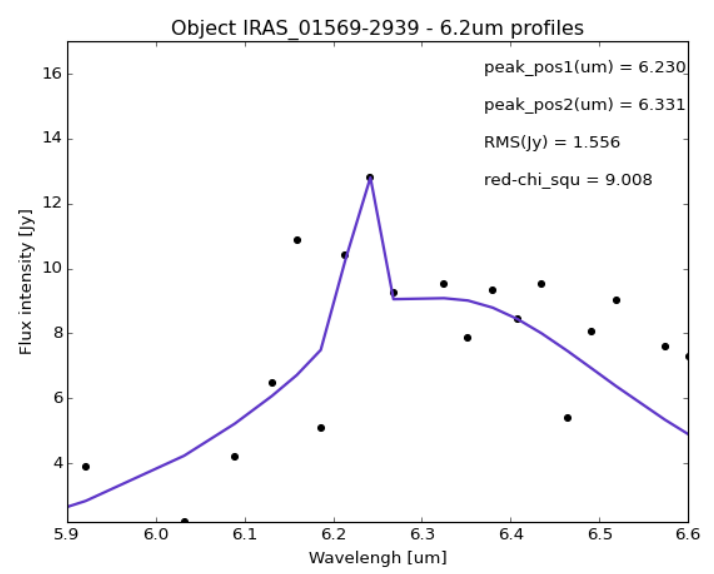

Figura C.64: Perfil da banda de $6.2 \mu m$ do objeto IRAS_01569-2939 ajustado com minimize. 


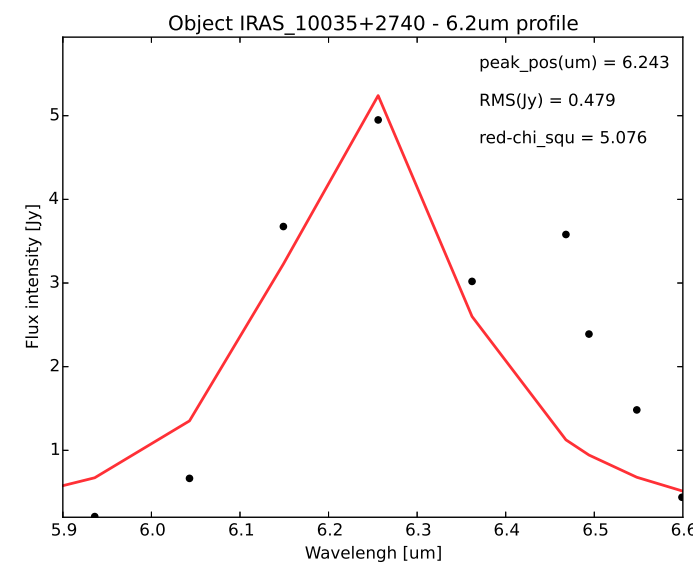

Figura C.65: Perfil da banda de $6.2 \mu m$ do objeto IRAS_10035+2740 ajustado com curve_fit e para $5.9 \leq \lambda \leq 6.4$.

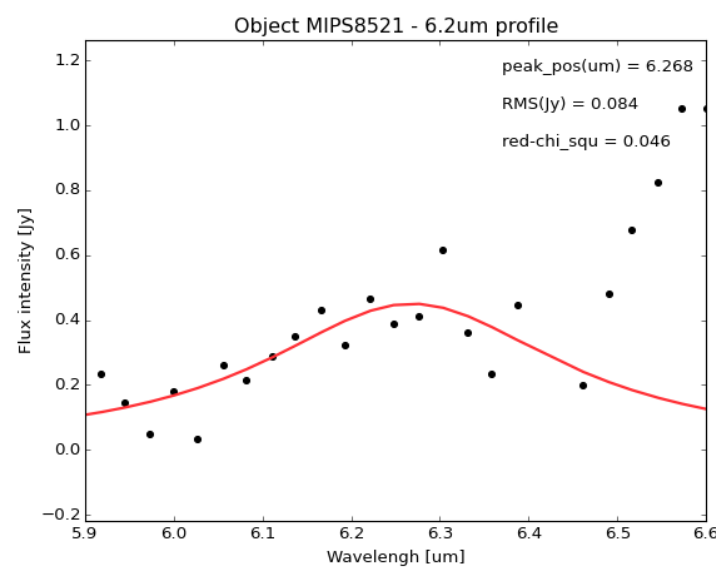

Figura C.67: Perfil da banda de $6.2 \mu \mathrm{m}$ do objeto MIPS8521 ajustado com curve_fit.

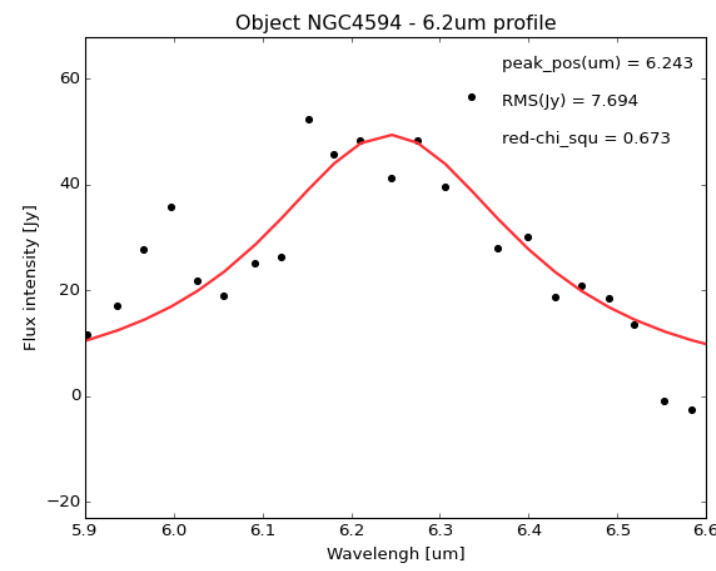

Figura C.69: Perfil da banda de $6.2 \mu \mathrm{m}$ do objeto NGC4594 ajustado com curve_fit.

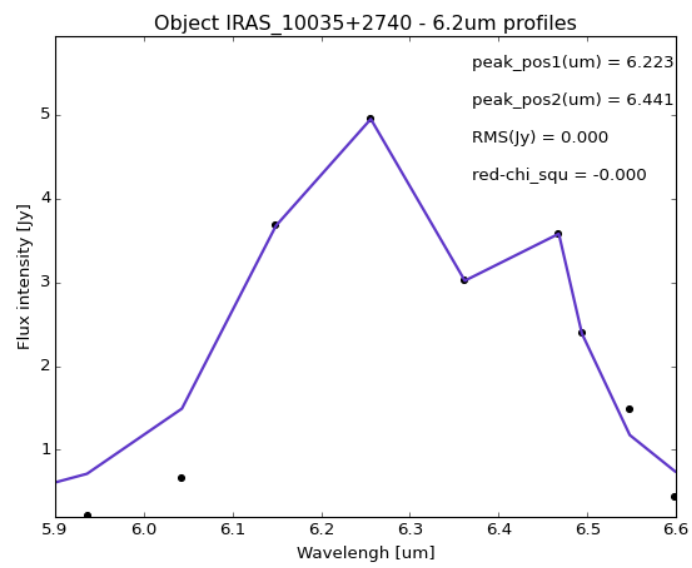

Figura C.66: Perfil da banda de $6.2 \mu \mathrm{m}$ do objeto IRAS_10035+2740 ajustado com minimize.

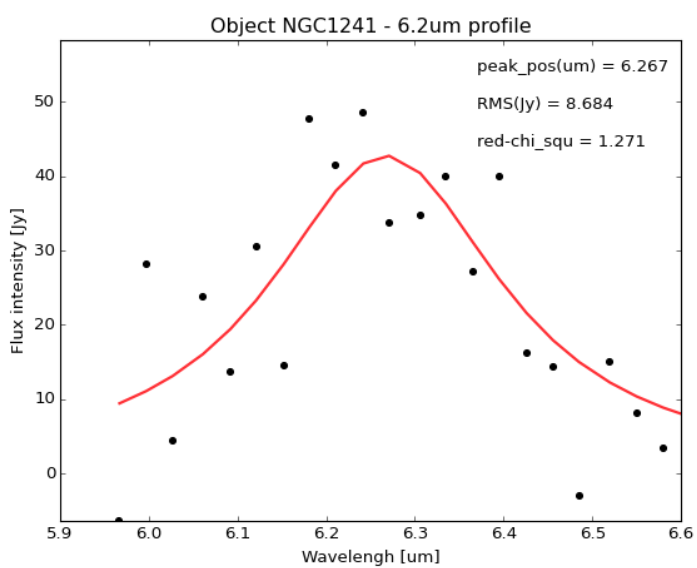

Figura C.68: Perfil da banda de $6.2 \mu m$ do objeto NGC1241 ajustado com curve_fit.

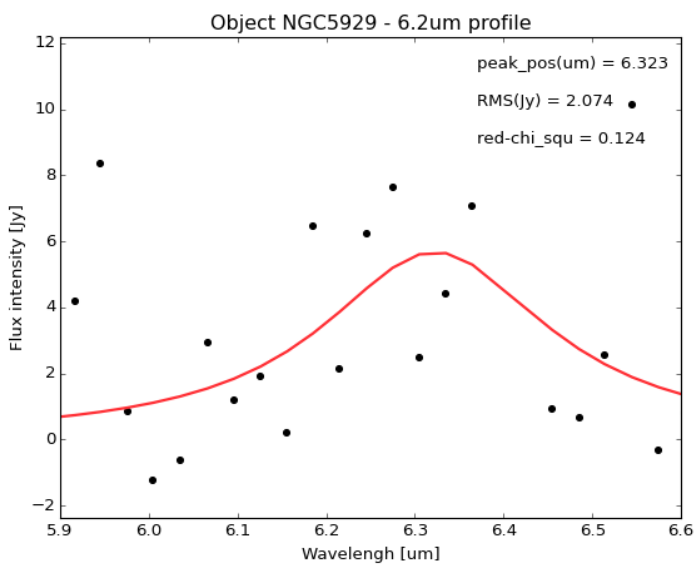

Figura C.70: Perfil da banda de $6.2 \mu \mathrm{m}$ do objeto NGC5929 ajustado com curve_fit. 


\section{Apêndice D}

\section{Fontes: identificação e propriedades derivadas}

Na segunda coluna da Tabela D.1 estão indicados os tipos das fontes, lembrando que elas foram extraídas da amostra $M I R_{-} S B$, cuja emissão dominante provém de starburst e mais algumas de Yan et al. (2007) (ver Seção 2.2 para maiores detalhes). As siglas são explicadas abaixo:

AGN Núcleo ativo de galáxia

FR galáxia Fanaroff-Riley

HII Região HII

IRgal Galáxia no infravermelho

LINER Regiões de emissão nuclear de baixa ionização

QSO Objeto quasi-estelar

SB galáxia Starburst

Sy galáxia Seyfert

SMG Galáxia no submilimétrico

ULIRG Galáxia no infravermelho ultra-luminosa 


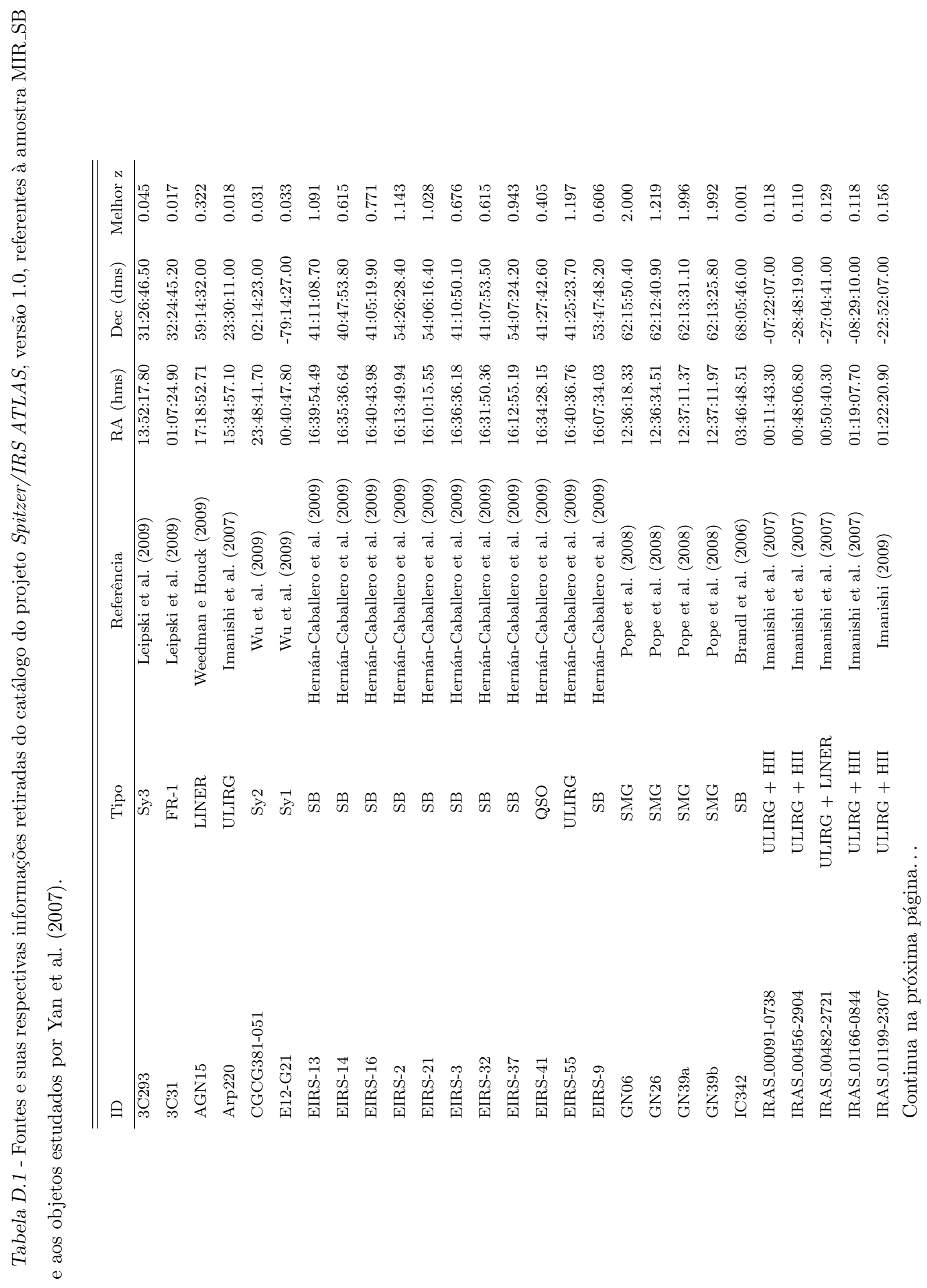




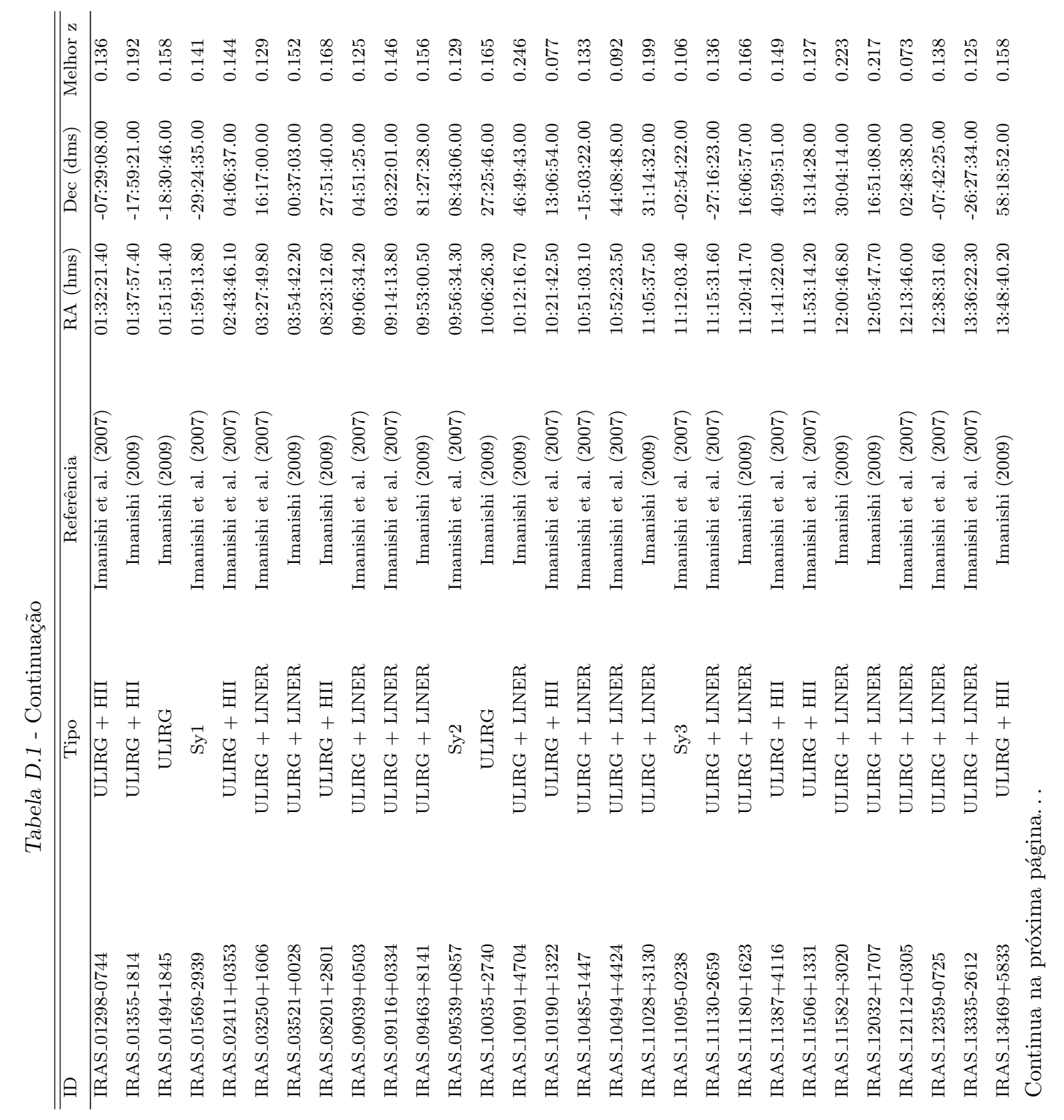




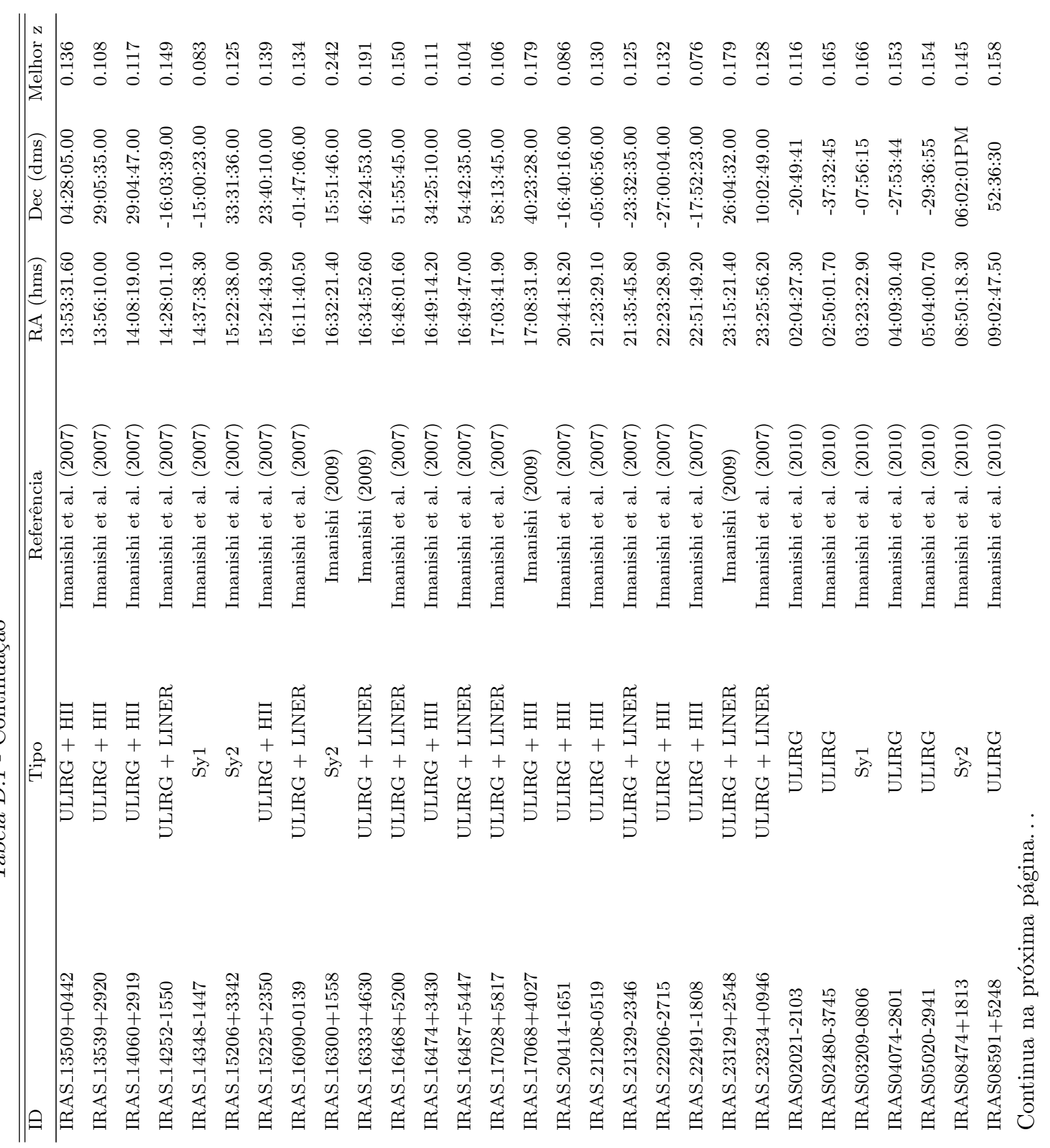




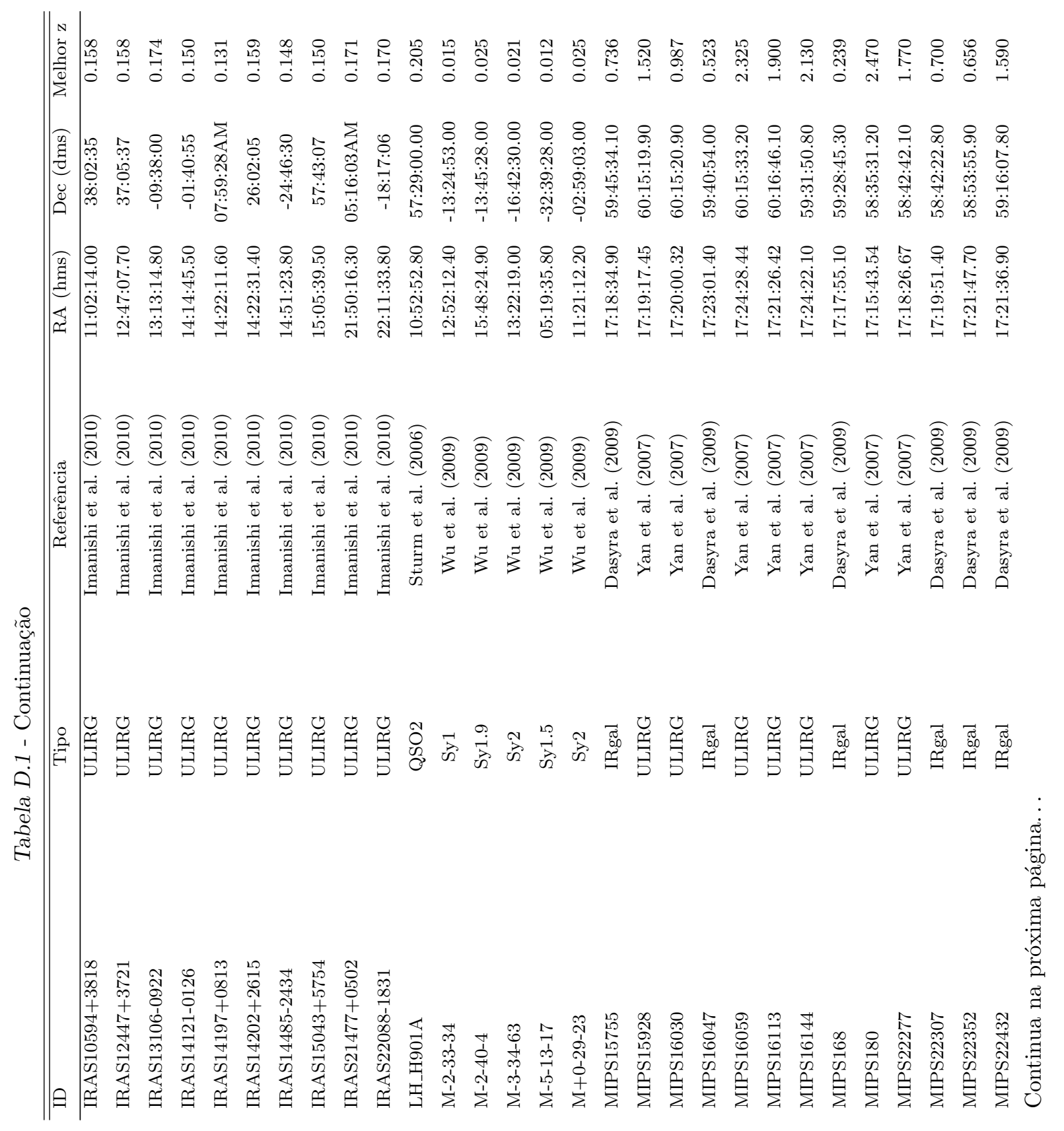




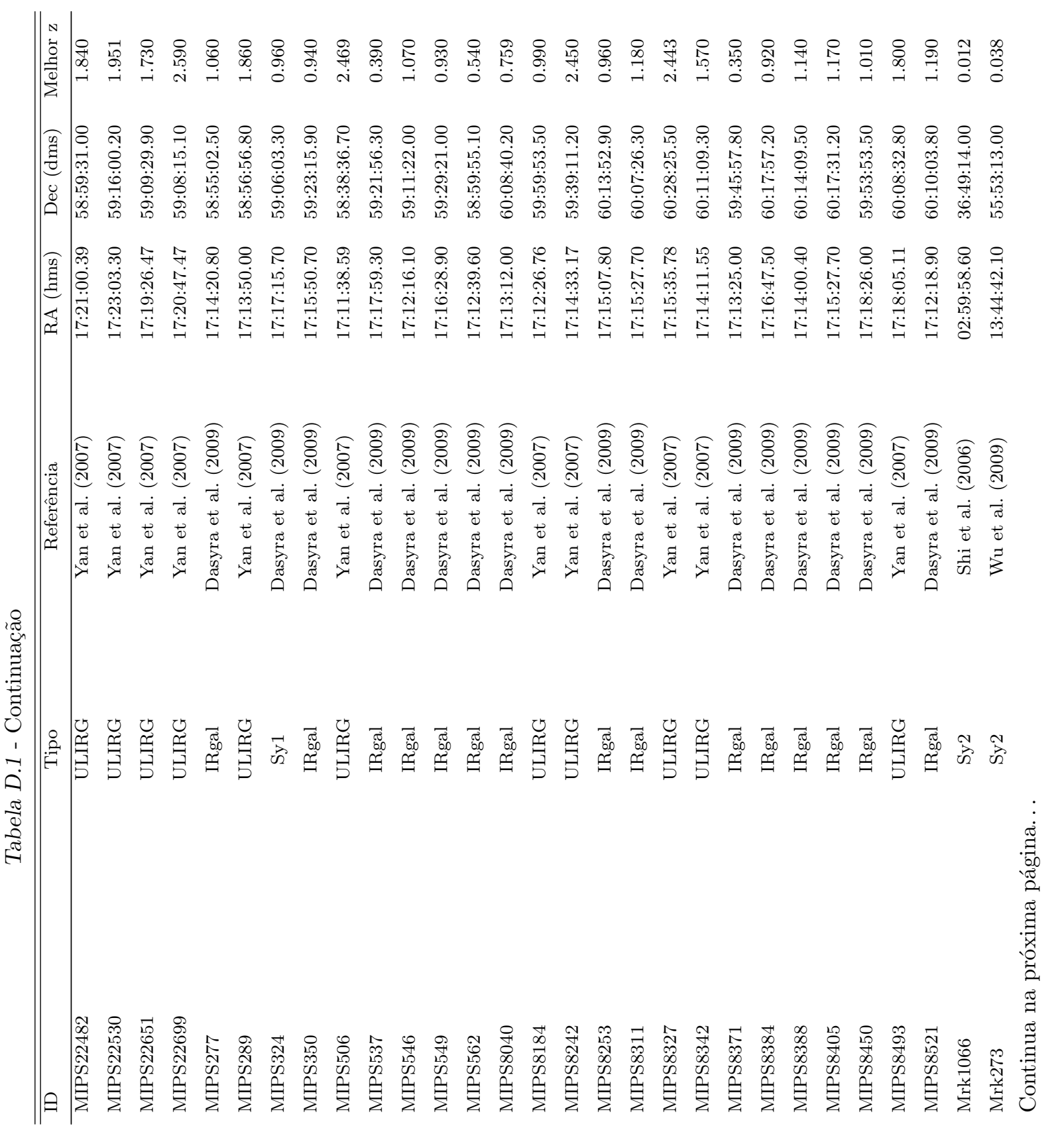




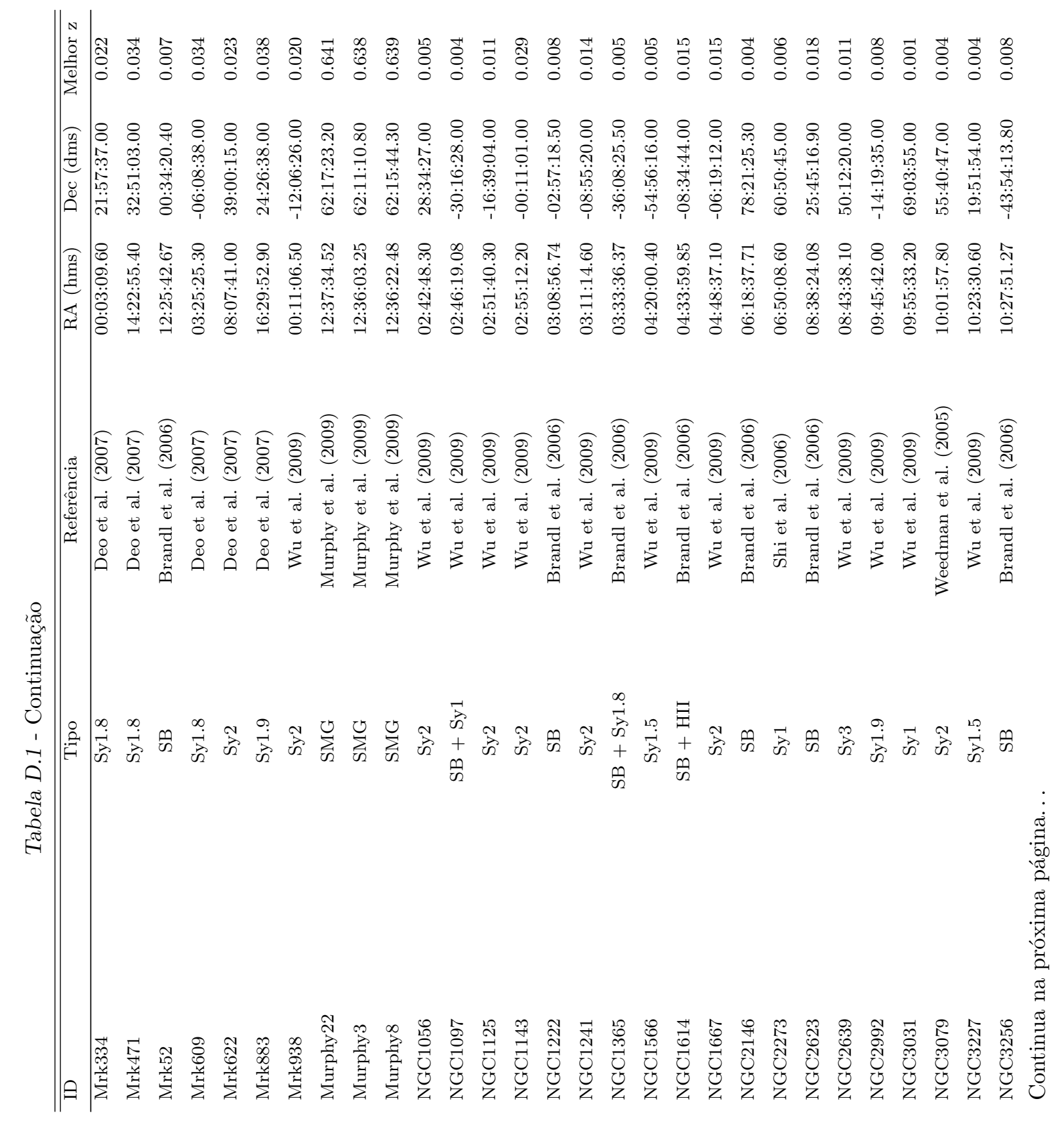




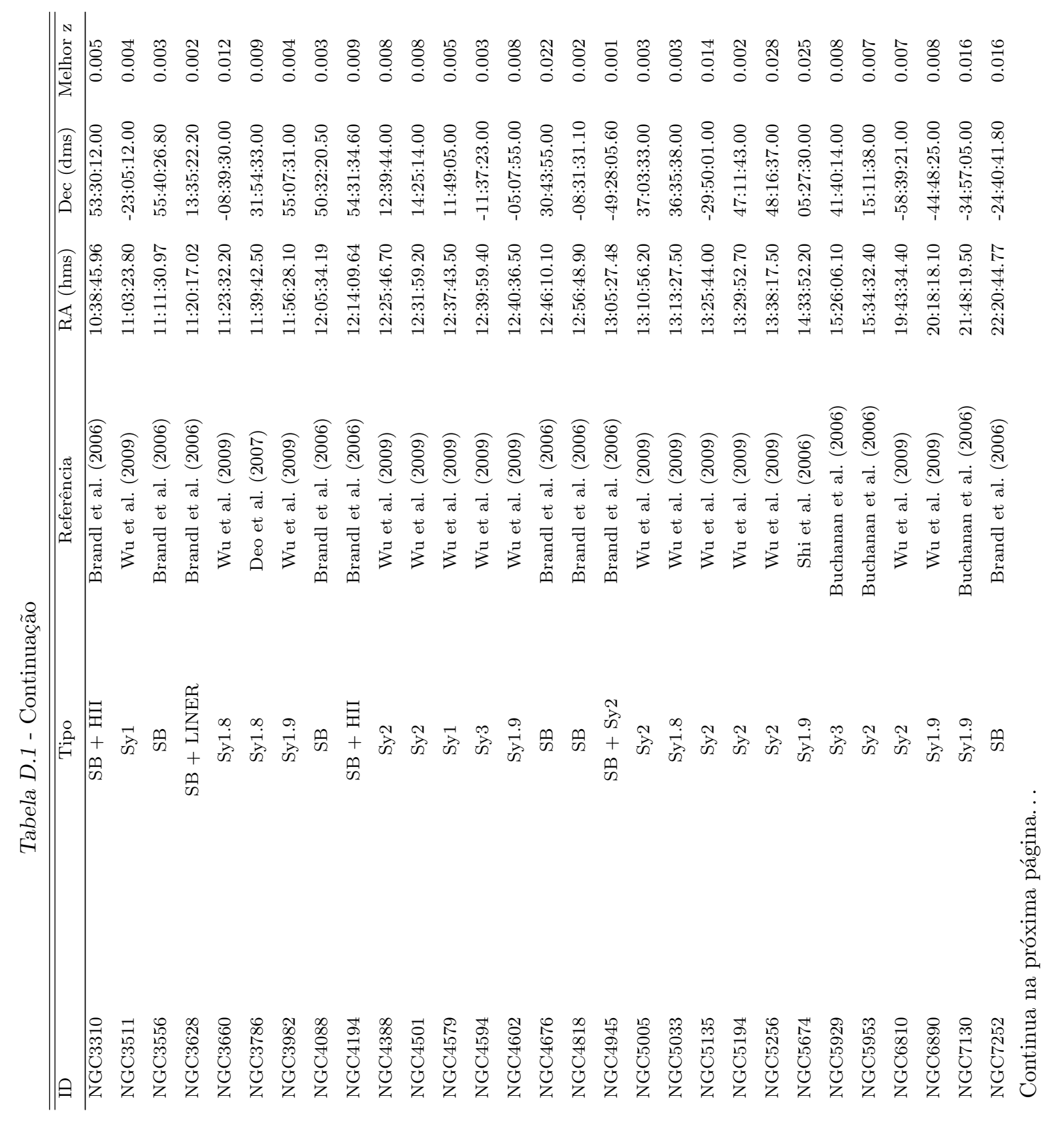




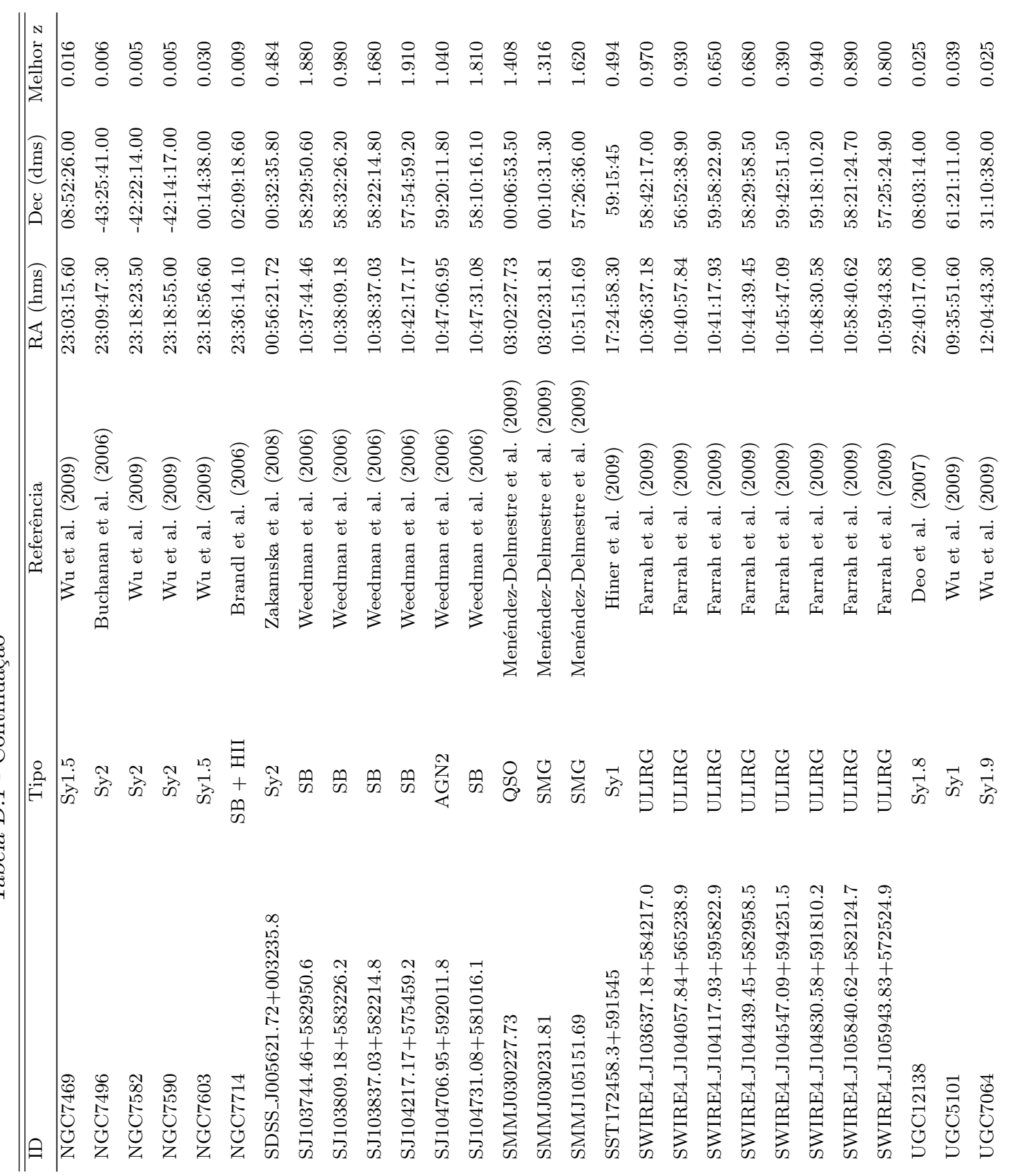


Tabela D.2 - Resultados parcias e totais de RMS.

\begin{tabular}{|c|c|c|c|}
\hline Objeto & Redshift & RMS total & RMS parcial \\
\hline 3C293 & 0.045 & 2.670 & 0.496 \\
\hline $3 \mathrm{C} 31$ & 0.017 & 1.441 & 0.712 \\
\hline AGN15 & 0.322 & 1.725 & 0.727 \\
\hline Arp220 & 0.018 & 288.388 & 22.281 \\
\hline CGCG381-051 & 0.031 & 11.165 & 8.505 \\
\hline E12-G21 & 0.033 & 6.741 & 6.206 \\
\hline EIRS-13 & 1.091 & 0.308 & 0.266 \\
\hline EIRS-14 & 0.615 & 0.553 & 0.243 \\
\hline EIRS-16 & 0.771 & 0.282 & 0.198 \\
\hline EIRS-2 & 1.143 & 0.263 & 0.181 \\
\hline EIRS-21 & 1.028 & 0.333 & 0.260 \\
\hline EIRS-3 & 0.676 & 0.419 & 0.203 \\
\hline EIRS-32 & 0.615 & 0.774 & 0.284 \\
\hline EIRS-37 & 0.943 & 0.388 & 0.354 \\
\hline EIRS-41 & 0.405 & 0.536 & 0.286 \\
\hline EIRS-55 & 1.197 & 0.504 & 0.404 \\
\hline EIRS-9 & 0.606 & 0.415 & 0.182 \\
\hline GN06 & 2.000 & 0.119 & 0.119 \\
\hline GN26 & 1.219 & 0.188 & 0.167 \\
\hline GN39a & 1.996 & 0.121 & 0.121 \\
\hline GN39b & 1.992 & 0.126 & 0.126 \\
\hline IC342 & 0.001 & 675.556 & 15.788 \\
\hline IRAS_00091-0738 & 0.118 & 10.304 & 2.424 \\
\hline IRAS_00456-2904 & 0.110 & 3.979 & 1.838 \\
\hline IRAS_00482-2721 & 0.129 & 2.924 & 0.597 \\
\hline IRAS_01166-0844 & 0.118 & 6.717 & 1.459 \\
\hline IRAS_01199-2307 & 0.156 & 5.868 & 1.355 \\
\hline IRAS_01298-0744 & 0.136 & 12.246 & 3.194 \\
\hline IRAS_01355-1814 & 0.192 & 5.165 & 0.673 \\
\hline IRAS_01494-1845 & 0.158 & 2.576 & 0.824 \\
\hline IRAS_01569-2939 & 0.141 & 5.908 & 1.651 \\
\hline IRAS_02411+0353 & 0.144 & 4.314 & 1.706 \\
\hline IRAS_03250+1606 & 0.129 & 3.364 & 1.231 \\
\hline IRAS_03521+0028 & 0.152 & 4.593 & 0.895 \\
\hline IRAS_08201+2801 & 0.168 & 5.497 & 1.605 \\
\hline IRAS_09039+0503 & 0.125 & 3.180 & 0.705 \\
\hline IRAS_09116+0334 & 0.146 & 2.110 & 0.913 \\
\hline IRAS_09463+8141 & 0.156 & 2.365 & 0.474 \\
\hline IRAS_09539+0857 & 0.129 & 5.613 & 1.066 \\
\hline IRAS_10035+2740 & 0.165 & 3.170 & 0.544 \\
\hline IRAS_10091+4704 & 0.246 & 2.476 & 0.756 \\
\hline IRAS_10190+1322 & 0.077 & 4.681 & 2.396 \\
\hline IRAS_10485-1447 & 0.133 & 5.161 & 1.242 \\
\hline
\end{tabular}


Tabela D.2 - Continuação

\begin{tabular}{|c|c|c|c|}
\hline Objeto & Redshift & RMS total & RMS parcial \\
\hline IRAS_10494+4424 & 0.092 & 3.588 & 1.702 \\
\hline IRAS_11028+3130 & 0.199 & 2.675 & 0.436 \\
\hline IRAS_11095-0238 & 0.106 & 17.558 & 4.264 \\
\hline IRAS_11130-2659 & 0.136 & 5.485 & 1.367 \\
\hline IRAS_11180+1623 & 0.166 & 3.469 & 1.009 \\
\hline IRAS_11387+4116 & 0.149 & 1.663 & 0.699 \\
\hline IRAS_11506+1331 & 0.127 & 7.182 & 2.081 \\
\hline IRAS_11582+3020 & 0.223 & 4.700 & 1.610 \\
\hline IRAS_12032+1707 & 0.217 & 4.326 & 1.662 \\
\hline IRAS_12112+0305 & 0.073 & 14.348 & 2.877 \\
\hline IRAS_12359-0725 & 0.138 & 4.215 & 0.912 \\
\hline IRAS_13335-2612 & 0.125 & 2.346 & 0.972 \\
\hline IRAS_13469+5833 & 0.158 & 2.131 & 0.878 \\
\hline IRAS_13509+0442 & 0.136 & 2.641 & 1.459 \\
\hline IRAS_13539+2920 & 0.108 & 3.285 & 1.727 \\
\hline IRAS_14060+2919 & 0.117 & 2.720 & 1.510 \\
\hline IRAS_14252-1550 & 0.149 & 1.668 & 0.695 \\
\hline IRAS_14348-1447 & 0.083 & 12.295 & 2.990 \\
\hline IRAS_15206+3342 & 0.125 & 5.513 & 2.491 \\
\hline IRAS_15225+2350 & 0.139 & 4.079 & 1.533 \\
\hline IRAS_16090-0139 & 0.134 & 8.276 & 3.445 \\
\hline IRAS_16300+1558 & 0.242 & 4.126 & 1.136 \\
\hline IRAS_16333+4630 & 0.191 & 2.560 & 0.907 \\
\hline IRAS_16468+5200 & 0.150 & 2.584 & 0.962 \\
\hline IRAS_16474+3430 & 0.111 & 3.554 & 2.356 \\
\hline IRAS_16487+5447 & 0.104 & 5.275 & 1.137 \\
\hline IRAS_17028+5817 & 0.106 & 2.910 & 1.104 \\
\hline IRAS_17068+4027 & 0.179 & 5.187 & 1.593 \\
\hline IRAS_20414-1651 & 0.086 & 7.569 & 1.220 \\
\hline IRAS_21208-0519 & 0.130 & 1.767 & 0.683 \\
\hline IRAS_21329-2346 & 0.125 & 3.286 & 0.814 \\
\hline IRAS_22206-2715 & 0.132 & 2.634 & 1.059 \\
\hline IRAS_22491-1808 & 0.076 & 21.165 & 3.079 \\
\hline IRAS_23129+2548 & 0.179 & 5.158 & 1.605 \\
\hline IRAS_23234+0946 & 0.128 & 3.428 & 0.819 \\
\hline IRAS02021-2103 & 0.116 & 5.159 & 1.373 \\
\hline IRAS02480-3745 & 0.165 & 3.116 & 1.025 \\
\hline IRAS03209-0806 & 0.166 & 2.064 & 0.905 \\
\hline IRAS04074-2801 & 0.153 & 6.141 & 1.711 \\
\hline IRAS05020-2941 & 0.154 & 6.771 & 1.713 \\
\hline IRAS08474+1813 & 0.145 & 3.751 & 0.919 \\
\hline IRAS08591+5248 & 0.158 & 2.085 & 1.213 \\
\hline IRAS10594+3818 & 0.158 & 1.984 & 1.261 \\
\hline IRAS12447+3721 & 0.158 & 2.975 & 0.946 \\
\hline
\end{tabular}

Continua na próxima página... 
Tabela D.2 - Continuação

\begin{tabular}{|c|c|c|c|}
\hline Objeto & Redshift & RMS total & RMS parcial \\
\hline IRAS13106-0922 & 0.174 & 2.633 & 1.454 \\
\hline IRAS14121-0126 & 0.150 & 2.633 & 1.775 \\
\hline IRAS14197+0813 & 0.131 & 2.867 & 1.150 \\
\hline IRAS14202+2615 & 0.159 & 3.170 & 1.824 \\
\hline IRAS14485-2434 & 0.148 & 3.339 & 1.175 \\
\hline IRAS15043+5754 & 0.150 & 2.074 & 0.992 \\
\hline IRAS21477+0502 & 0.171 & 2.864 & 0.738 \\
\hline IRAS22088-1831 & 0.170 & 5.734 & 1.051 \\
\hline LH_H901A & 0.205 & 0.077 & 0.077 \\
\hline M-2-33-34 & 0.015 & 8.732 & 7.150 \\
\hline M-2-40-4 & 0.025 & 12.028 & 8.522 \\
\hline M-3-34-63 & 0.021 & 5.954 & 5.470 \\
\hline M-5-13-17 & 0.012 & 7.788 & 8.215 \\
\hline$M+0-29-23$ & 0.025 & 11.749 & 7.632 \\
\hline MIPS15755 & 0.736 & 0.238 & 0.216 \\
\hline MIPS15928 & 1.520 & 0.144 & 0.068 \\
\hline MIPS16030 & 0.987 & 0.138 & 0.094 \\
\hline MIPS16047 & 0.523 & 0.234 & 0.183 \\
\hline MIPS16059 & 2.325 & 0.123 & 0.123 \\
\hline MIPS16113 & 1.900 & 0.140 & 0.136 \\
\hline MIPS16144 & 2.130 & 0.152 & 0.142 \\
\hline MIPS168 & 0.239 & 0.214 & 0.120 \\
\hline MIPS180 & 2.470 & 0.125 & 0.125 \\
\hline MIPS22277 & 1.770 & 0.158 & 0.140 \\
\hline MIPS22307 & 0.700 & 0.155 & 0.152 \\
\hline MIPS22352 & 0.656 & 0.153 & 0.156 \\
\hline MIPS22432 & 1.590 & 0.144 & 0.139 \\
\hline MIPS22482 & 1.840 & 0.128 & 0.100 \\
\hline MIPS22530 & 1.951 & 0.128 & 0.097 \\
\hline MIPS22651 & 1.730 & 0.198 & 0.085 \\
\hline MIPS22699 & 2.590 & 0.097 & 0.097 \\
\hline MIPS277 & 1.060 & 0.193 & 0.209 \\
\hline MIPS289 & 1.860 & 0.190 & 0.121 \\
\hline MIPS324 & 0.960 & 0.169 & 0.142 \\
\hline MIPS350 & 0.940 & 0.214 & 0.188 \\
\hline MIPS506 & 2.469 & 0.101 & 0.101 \\
\hline MIPS537 & 0.390 & 0.176 & 0.100 \\
\hline MIPS546 & 1.070 & 0.167 & 0.158 \\
\hline MIPS549 & 0.930 & 0.180 & 0.207 \\
\hline MIPS562 & 0.540 & 0.172 & 0.129 \\
\hline MIPS8040 & 0.759 & 0.224 & 0.193 \\
\hline MIPS8184 & 0.990 & 0.177 & 0.080 \\
\hline MIPS8242 & 2.450 & 0.206 & 0.206 \\
\hline MIPS8253 & 0.960 & 0.177 & 0.152 \\
\hline
\end{tabular}

Continua na próxima página... 
Tabela D.2 - Continuação

\begin{tabular}{|c|c|c|c|}
\hline Objeto & Redshift & RMS total & RMS parcial \\
\hline MIPS8311 & 1.180 & 0.172 & 0.169 \\
\hline MIPS8327 & 2.443 & 0.181 & 0.181 \\
\hline MIPS8342 & 1.570 & 0.163 & 0.070 \\
\hline MIPS8371 & 0.350 & 0.186 & 0.104 \\
\hline MIPS8384 & 0.920 & 0.149 & 0.140 \\
\hline MIPS8388 & 1.140 & 0.200 & 0.193 \\
\hline MIPS8405 & 1.170 & 0.186 & 0.182 \\
\hline MIPS8450 & 1.010 & 0.197 & 0.215 \\
\hline MIPS8493 & 1.800 & 0.125 & 0.084 \\
\hline MIPS8521 & 1.190 & 0.230 & 0.169 \\
\hline Mrk1066 & 0.012 & 22.851 & 8.093 \\
\hline Mrk273 & 0.038 & 42.957 & 8.152 \\
\hline Mrk334 & 0.022 & 10.819 & 6.782 \\
\hline Mrk471 & 0.034 & 1.946 & 1.582 \\
\hline Mrk52 & 0.007 & 37.602 & 4.024 \\
\hline Mrk609 & 0.034 & 6.800 & 2.236 \\
\hline Mrk622 & 0.023 & 4.002 & 1.356 \\
\hline Mrk883 & 0.038 & 3.149 & 0.947 \\
\hline Mrk938 & 0.020 & 43.803 & 11.501 \\
\hline Murphy22 & 0.641 & 0.075 & 0.075 \\
\hline Murphy3 & 0.638 & 0.112 & 0.107 \\
\hline Murphy8 & 0.639 & 0.086 & 0.085 \\
\hline NGC1056 & 0.005 & 9.243 & 8.844 \\
\hline NGC1097 & 0.004 & 42.654 & 53.326 \\
\hline NGC1125 & 0.011 & 10.567 & 7.911 \\
\hline NGC1143 & 0.029 & 9.012 & 9.655 \\
\hline NGC1222 & 0.008 & 71.973 & 5.436 \\
\hline NGC1241 & 0.014 & 6.800 & 8.240 \\
\hline NGC1365 & 0.005 & 76.113 & 9.382 \\
\hline NGC1566 & 0.005 & 12.882 & 13.780 \\
\hline NGC1614 & 0.015 & 185.765 & 19.488 \\
\hline NGC1667 & 0.015 & 7.565 & 7.881 \\
\hline NGC2146 & 0.004 & 604.133 & 59.487 \\
\hline NGC2273 & 0.006 & 13.430 & 6.770 \\
\hline NGC2623 & 0.018 & 173.568 & 6.478 \\
\hline NGC2639 & 0.011 & 6.994 & 7.454 \\
\hline NGC2992 & 0.008 & 17.586 & 8.796 \\
\hline NGC3031 & 0.001 & 31.884 & 35.281 \\
\hline NGC3079 & 0.004 & 29.817 & 19.650 \\
\hline NGC3227 & 0.004 & 29.743 & 10.108 \\
\hline NGC3256 & 0.008 & 275.477 & 73.307 \\
\hline NGC3310 & 0.005 & 84.034 & 7.949 \\
\hline NGC3511 & 0.004 & 5.943 & 7.331 \\
\hline NGC3556 & 0.003 & 43.440 & 12.710 \\
\hline
\end{tabular}

Continua na próxima página... 
Tabela D.2 - Continuação

\begin{tabular}{|c|c|c|c|}
\hline Objeto & Redshift & RMS total & RMS parcial \\
\hline NGC3628 & 0.002 & 47.267 & 18.450 \\
\hline NGC3660 & 0.012 & 6.540 & 7.187 \\
\hline NGC3786 & 0.009 & 3.739 & 2.530 \\
\hline NGC3982 & 0.004 & 8.425 & 8.941 \\
\hline NGC4088 & 0.003 & 18.695 & 3.437 \\
\hline NGC4194 & 0.009 & 171.926 & 15.427 \\
\hline NGC4388 & 0.008 & 33.772 & 11.529 \\
\hline NGC4501 & 0.008 & 6.629 & 7.825 \\
\hline NGC4579 & 0.005 & 11.077 & 15.263 \\
\hline NGC4594 & 0.003 & 7.825 & 10.560 \\
\hline NGC4602 & 0.008 & 6.110 & 6.328 \\
\hline NGC4676 & 0.022 & 12.806 & 2.409 \\
\hline NGC4818 & 0.002 & 135.281 & 10.650 \\
\hline NGC4945 & 0.001 & 2204.977 & 84.899 \\
\hline NGC5005 & 0.003 & 11.007 & 9.529 \\
\hline NGC5033 & 0.003 & 22.754 & 25.822 \\
\hline NGC5135 & 0.014 & 36.372 & 10.488 \\
\hline NGC5194 & 0.002 & 30.069 & 27.422 \\
\hline NGC5256 & 0.028 & 12.977 & 7.652 \\
\hline NGC5674 & 0.025 & 0.826 & 0.760 \\
\hline NGC5929 & 0.008 & 14.341 & 3.167 \\
\hline NGC5953 & 0.007 & 16.252 & 9.301 \\
\hline NGC6810 & 0.007 & 33.264 & 12.370 \\
\hline NGC6890 & 0.008 & 8.030 & 7.583 \\
\hline NGC7130 & 0.016 & 26.020 & 11.688 \\
\hline NGC7252 & 0.016 & 21.373 & 4.906 \\
\hline NGC7469 & 0.016 & 65.468 & 13.111 \\
\hline NGC7496 & 0.006 & 51.277 & 12.181 \\
\hline NGC7582 & 0.005 & 74.800 & 21.245 \\
\hline NGC7590 & 0.005 & 6.737 & 8.134 \\
\hline NGC7603 & 0.030 & 7.479 & 8.679 \\
\hline NGC7714 & 0.009 & 78.229 & 7.019 \\
\hline SDSS_J005621.72+003235.8 & 0.484 & 0.561 & 0.503 \\
\hline SJ103744.46+582950.6 & 1.880 & 0.165 & 0.165 \\
\hline SJ103809.18+583226.2 & 0.980 & 0.154 & 0.151 \\
\hline SJ103837.03+582214.8 & 1.680 & 0.216 & 0.218 \\
\hline SJ104217.17+575459.2 & 1.910 & 0.145 & 0.145 \\
\hline SJ104706.95+592011.8 & 1.040 & 0.158 & 0.161 \\
\hline SJ104731.08+581016.1 & 1.810 & 0.180 & 0.180 \\
\hline SMMJ030227.73 & 1.408 & 0.066 & 0.064 \\
\hline SMMJ030231.81 & 1.316 & 0.155 & 0.153 \\
\hline SMMJ105151.69 & 1.620 & 0.053 & 0.053 \\
\hline SST172458.3+591545 & 0.494 & 0.246 & 0.107 \\
\hline SWIRE4_J103637.18+584217.0 & 0.970 & 0.189 & 0.160 \\
\hline
\end{tabular}

Continua na próxima página... 
Tabela D.2 - Continuação

\begin{tabular}{lccc}
\hline \hline Objeto & Redshift & RMS total & RMS parcial \\
\hline SWIRE4_J104057.84+565238.9 & 0.930 & 0.046 & 0.046 \\
SWIRE4_J104117.93+595822.9 & 0.650 & 0.069 & 0.069 \\
SWIRE4_J104439.45+582958.5 & 0.680 & 0.067 & 0.058 \\
SWIRE4_J104547.09+594251.5 & 0.390 & 0.073 & 0.049 \\
SWIRE4_J104830.58+591810.2 & 0.940 & 0.128 & 0.128 \\
SWIRE4_J105840.62+582124.7 & 0.890 & 0.207 & 0.164 \\
SWIRE4_J105943.83+572524.9 & 0.800 & 0.219 & 0.133 \\
UGC12138 & 0.025 & 3.853 & 1.639 \\
UGC5101 & 0.039 & 9.304 & 4.873 \\
UGC7064 & 0.025 & 8.116 & 8.826 \\
& & & \\
\hline
\end{tabular}




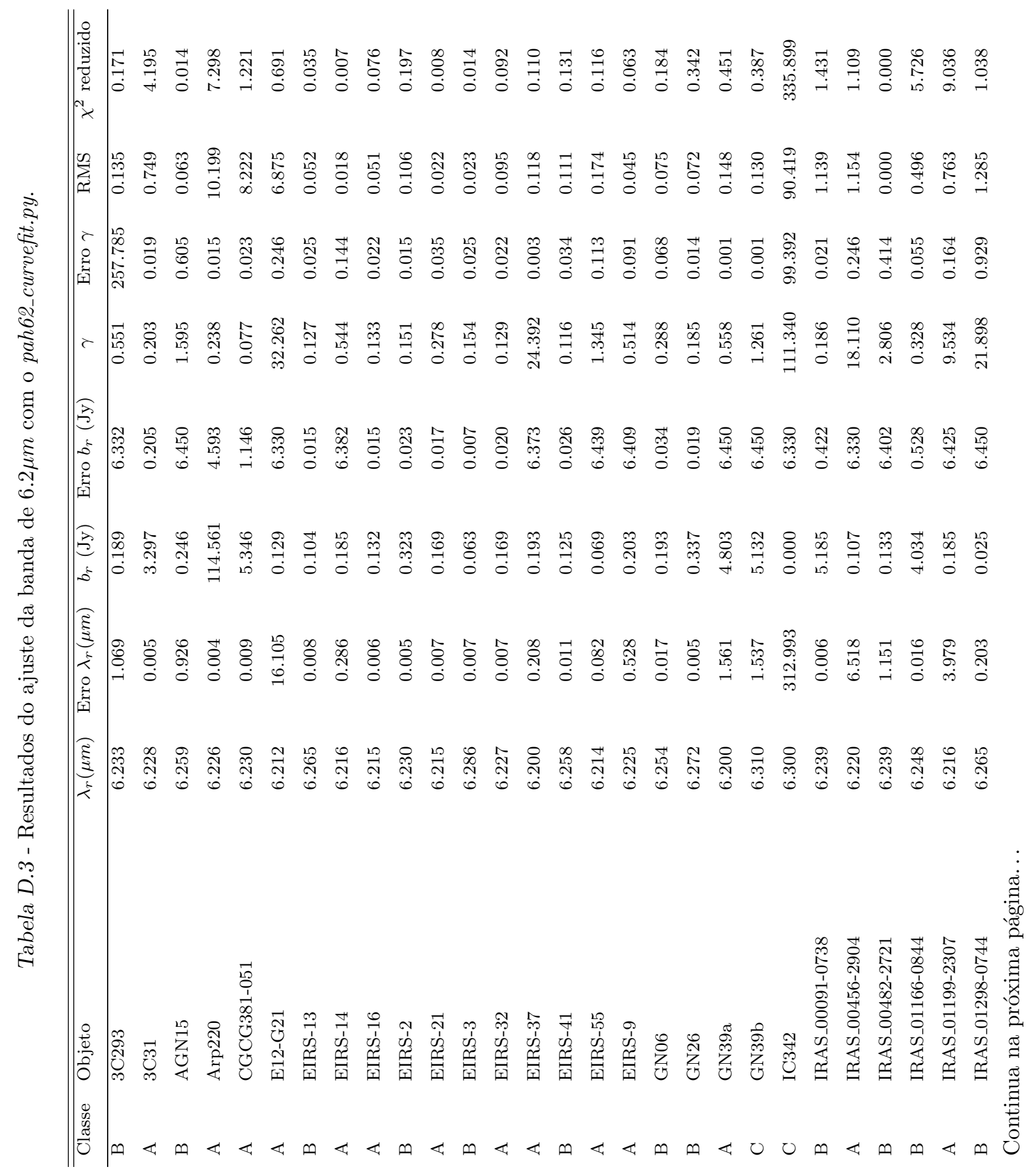




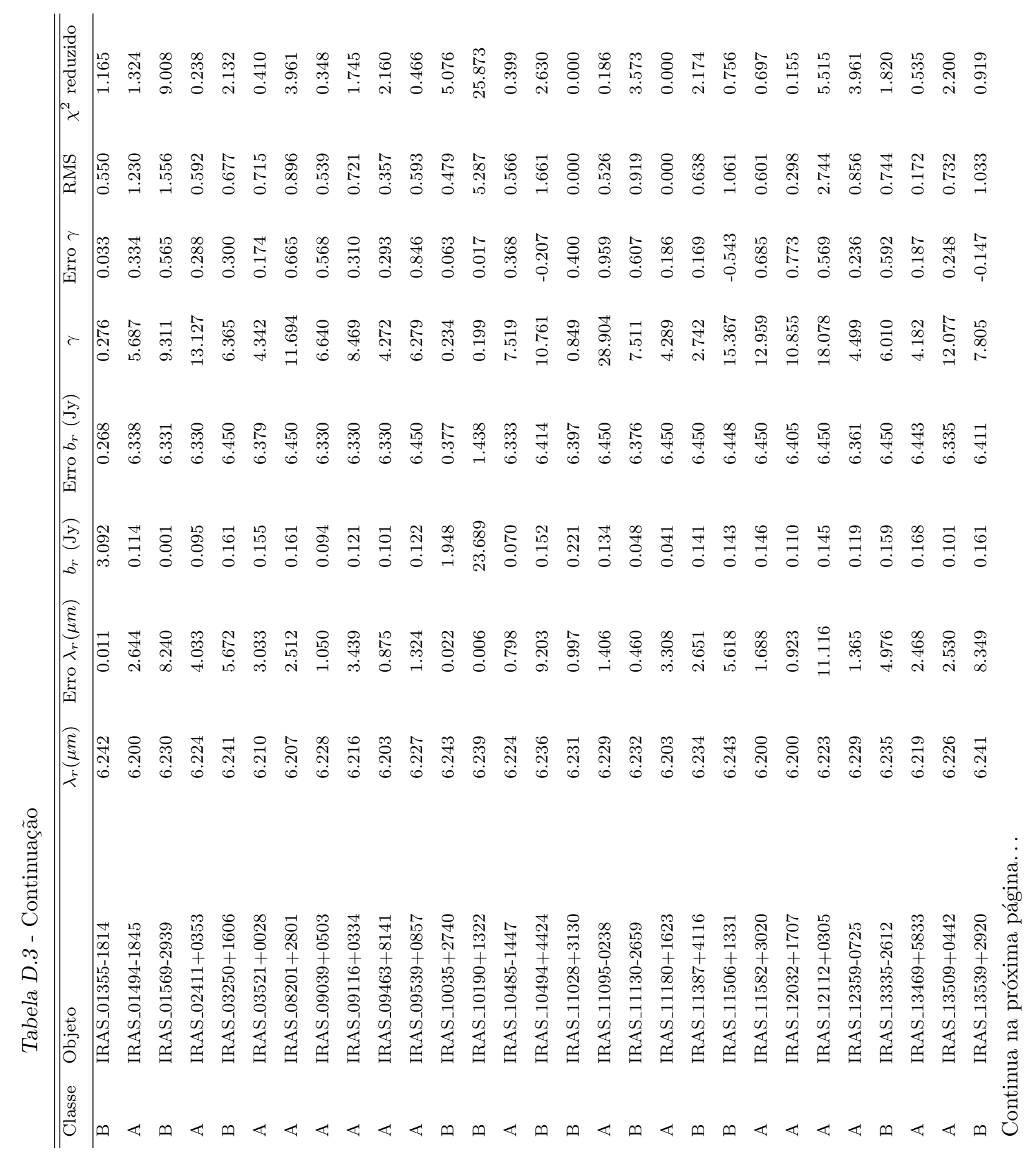




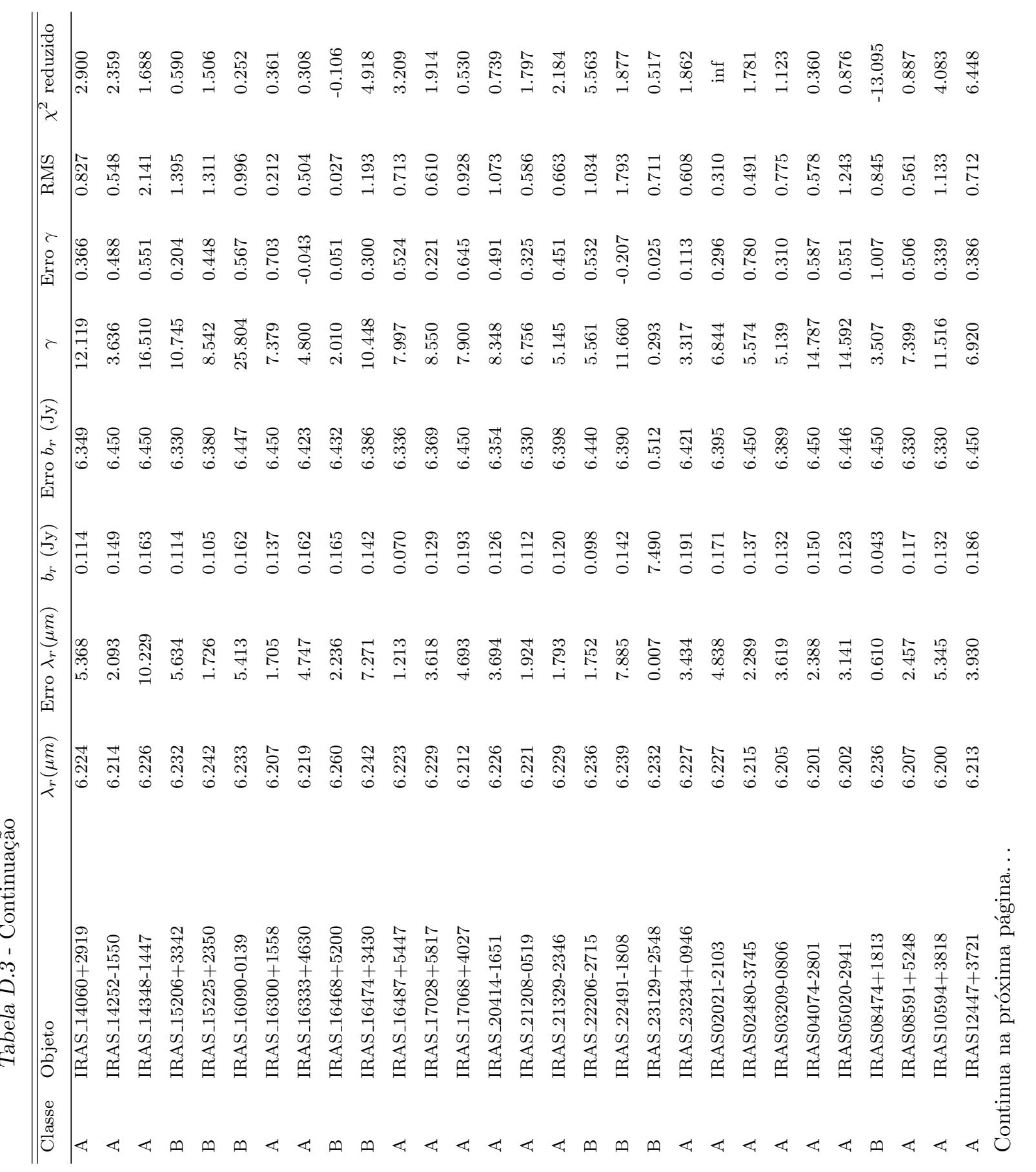




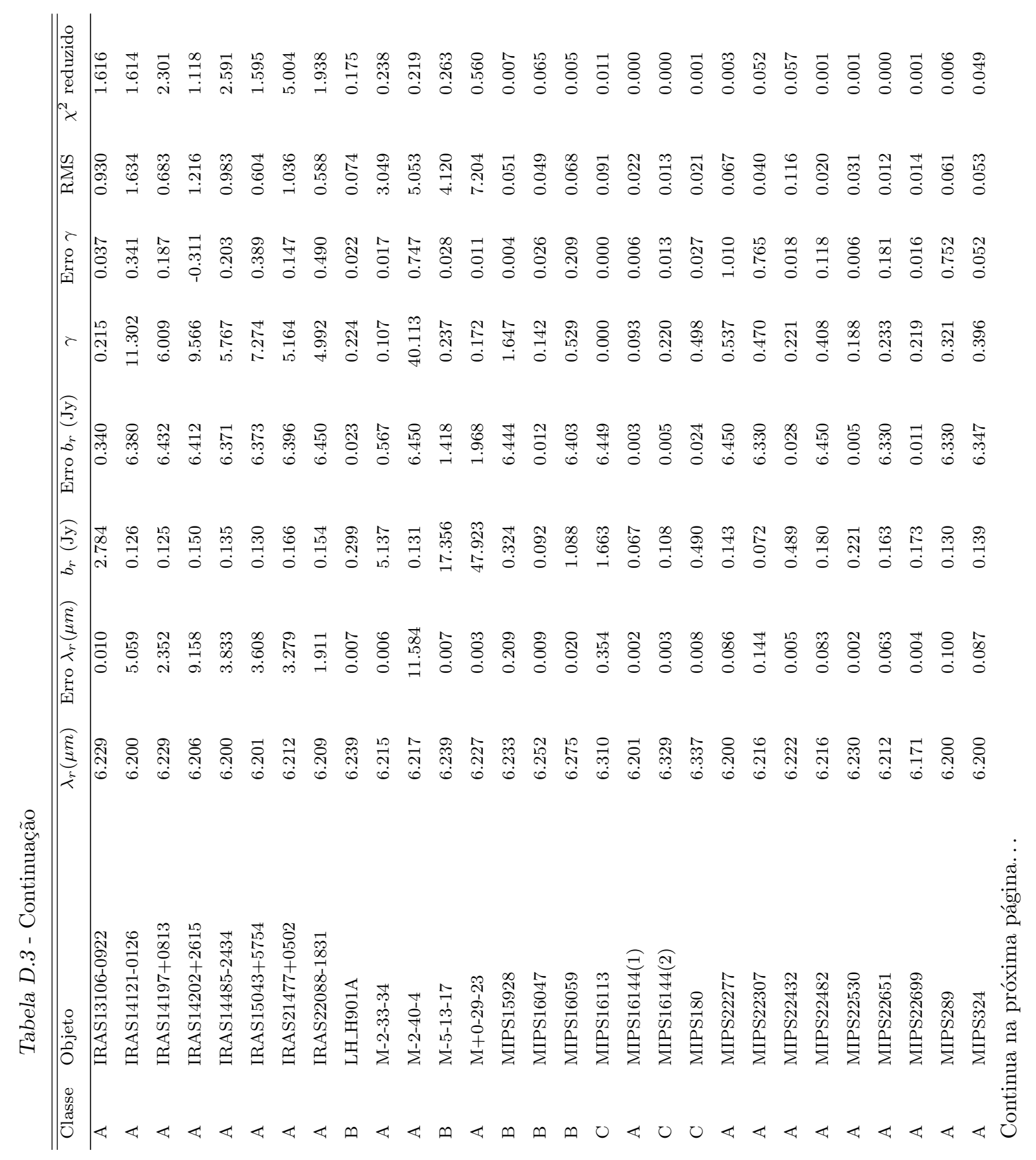




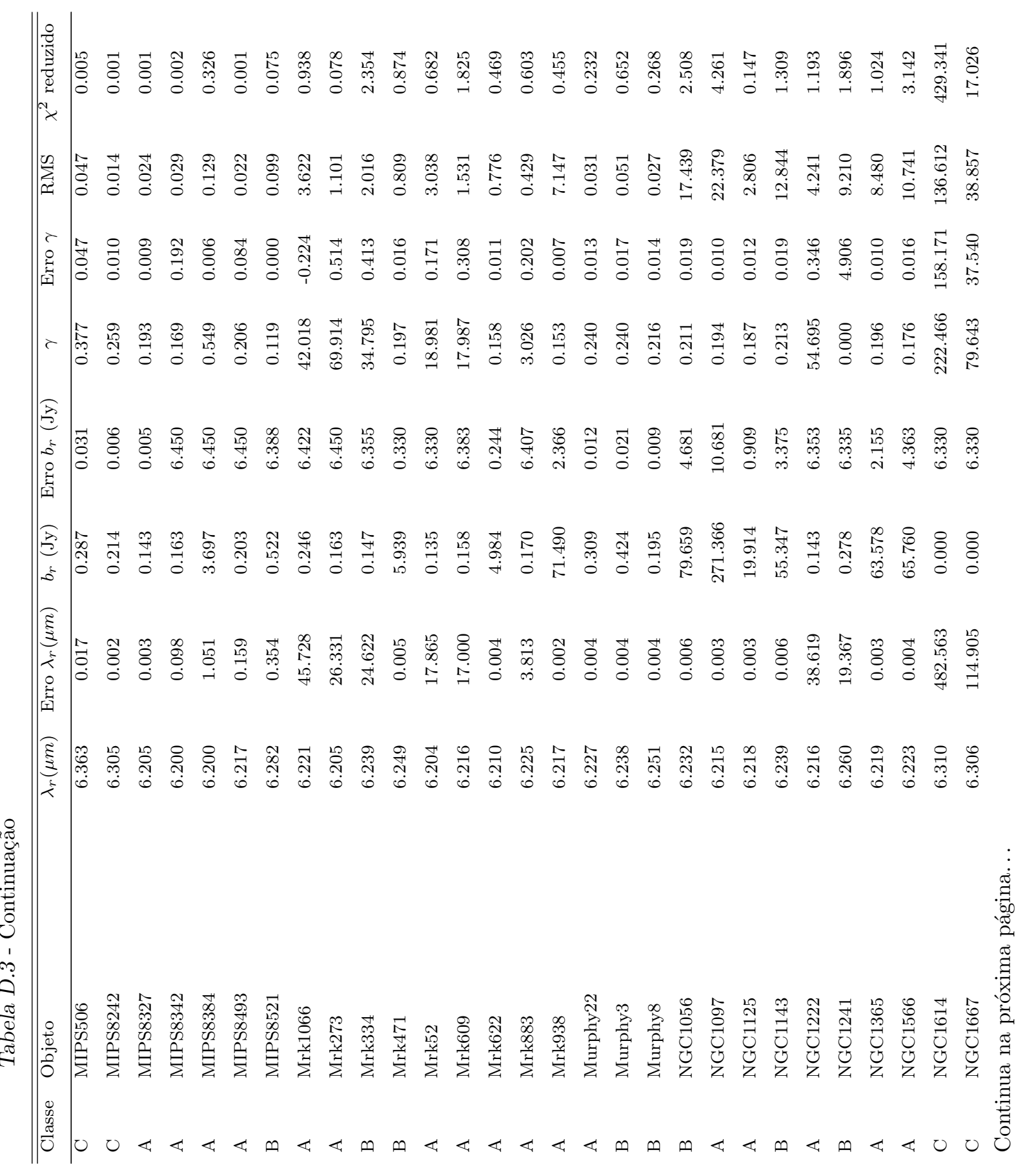




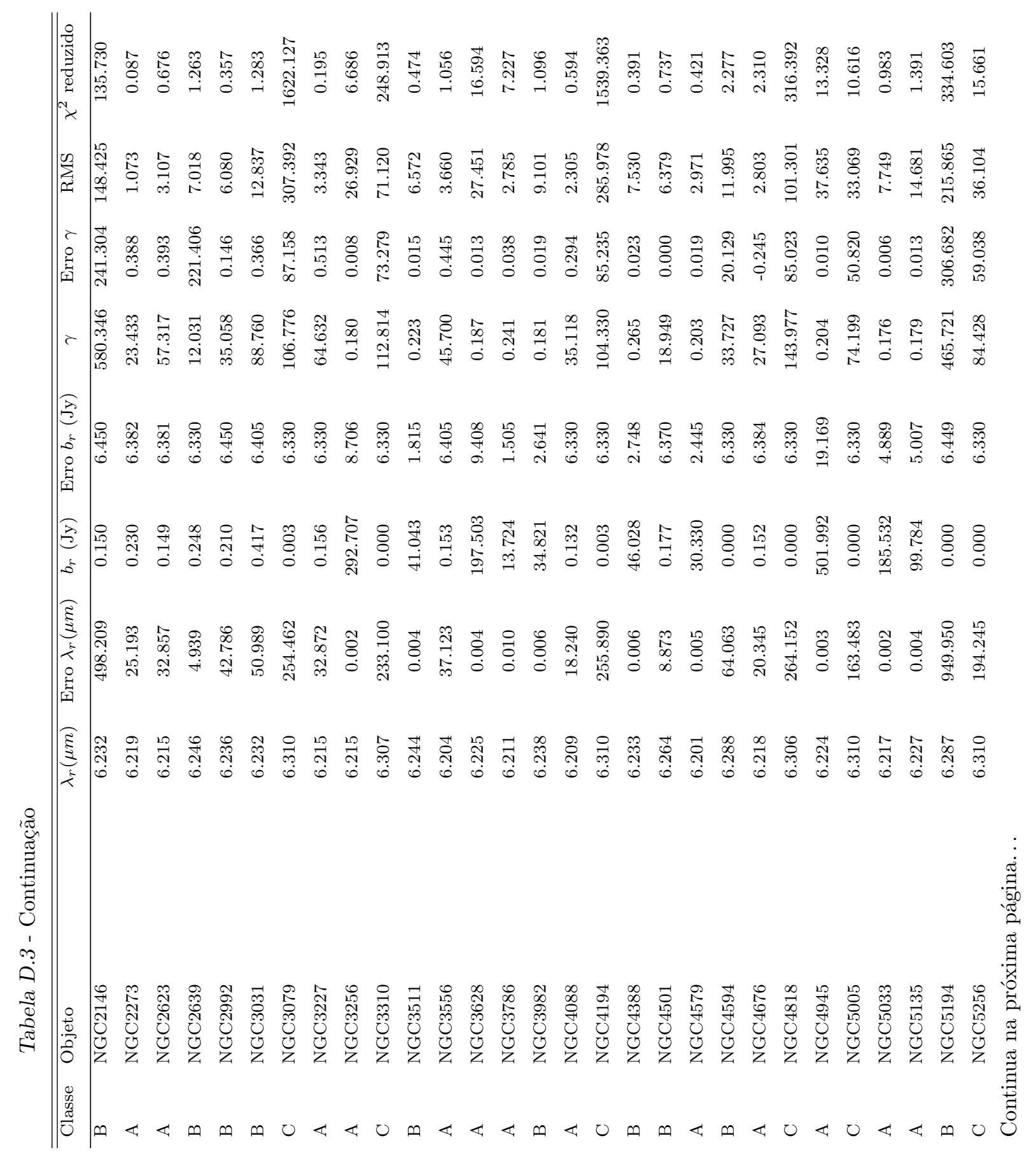




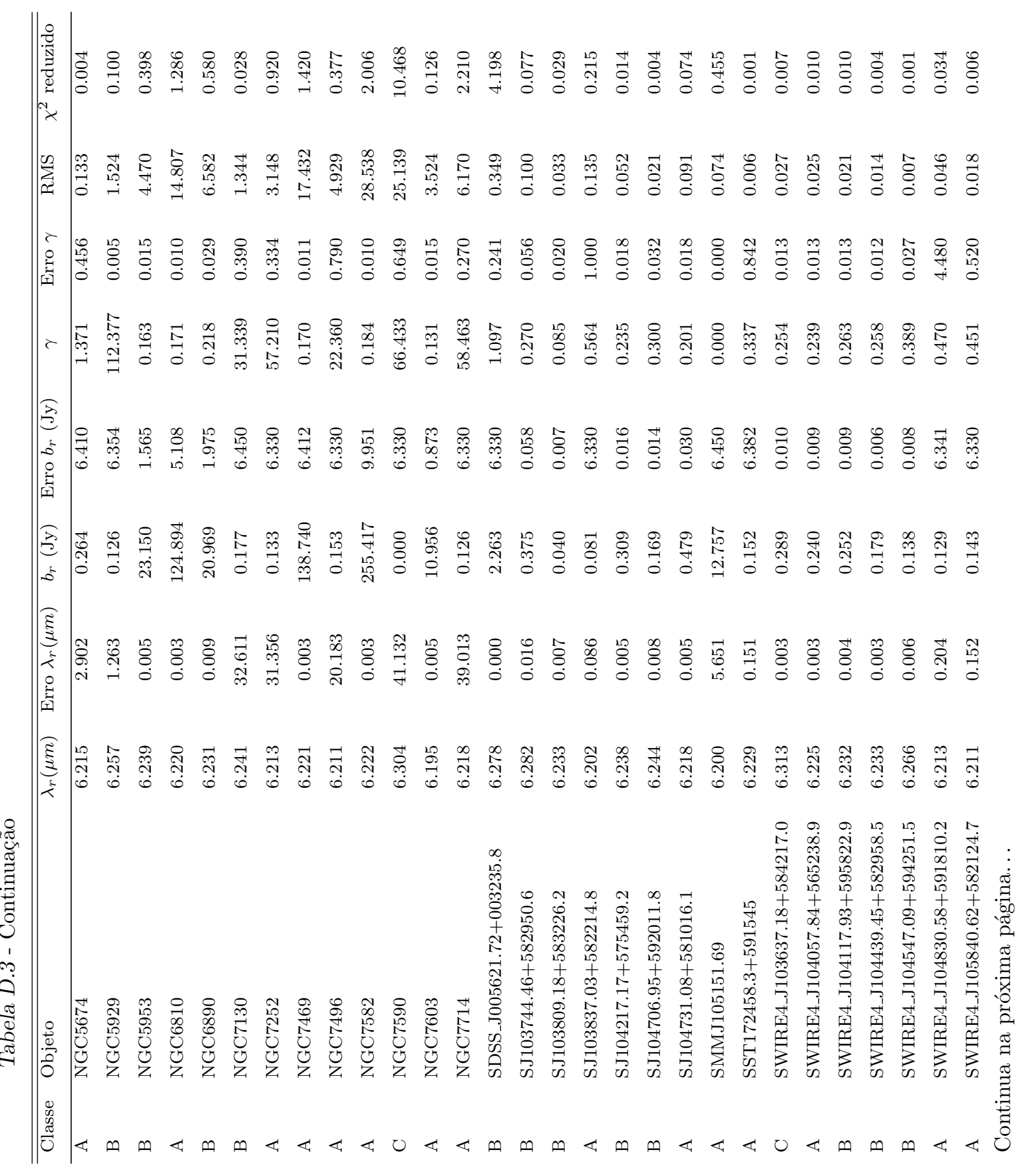




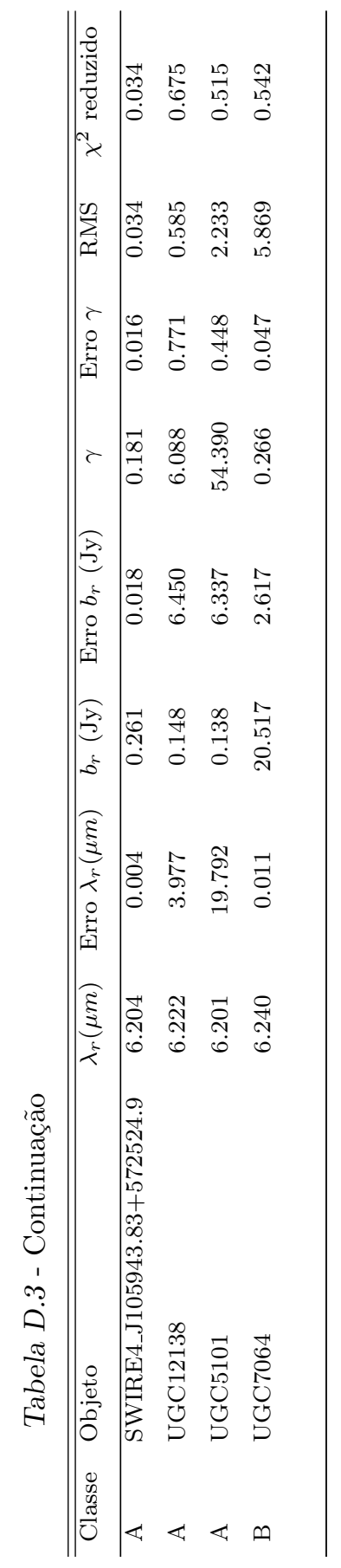




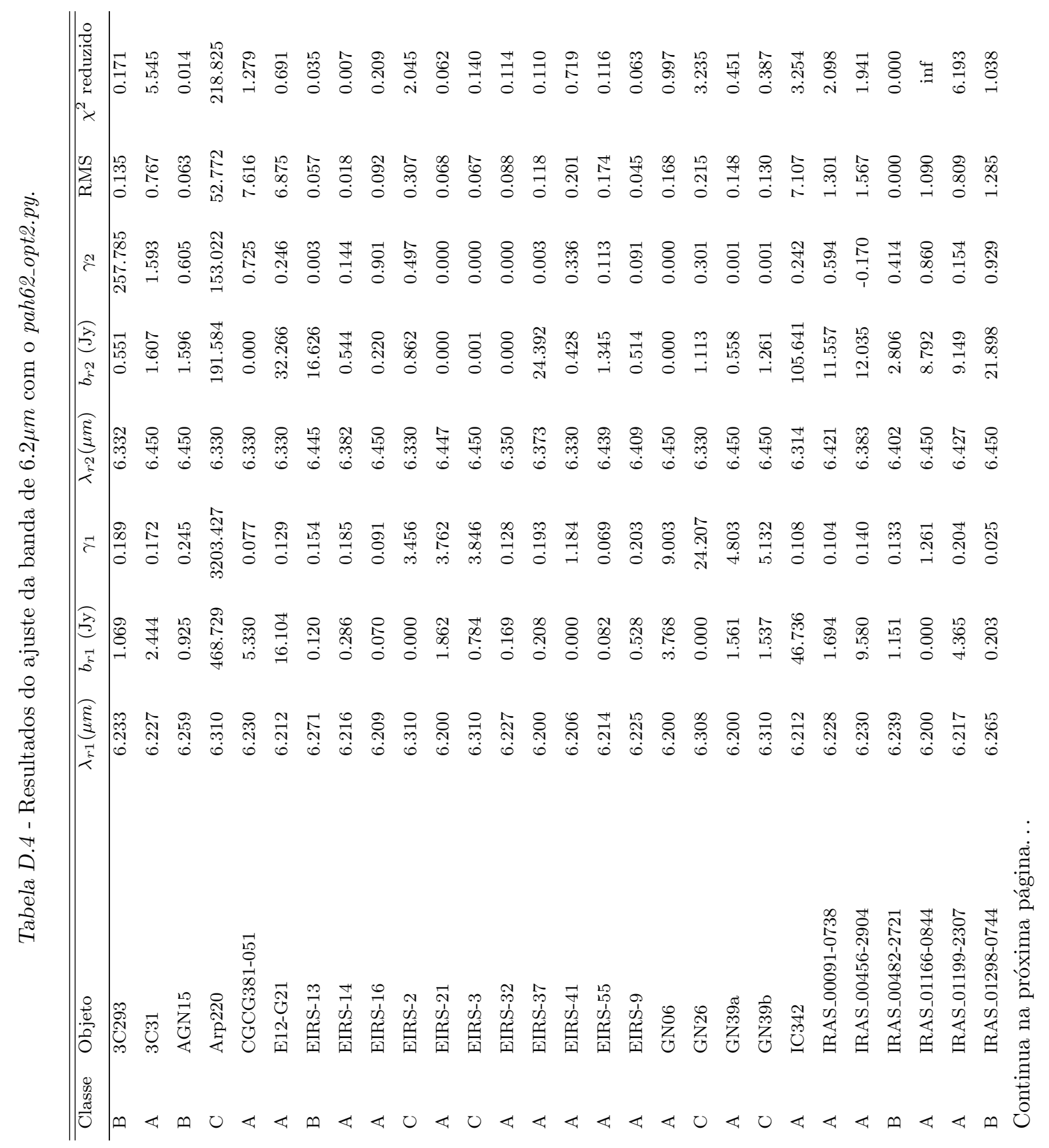




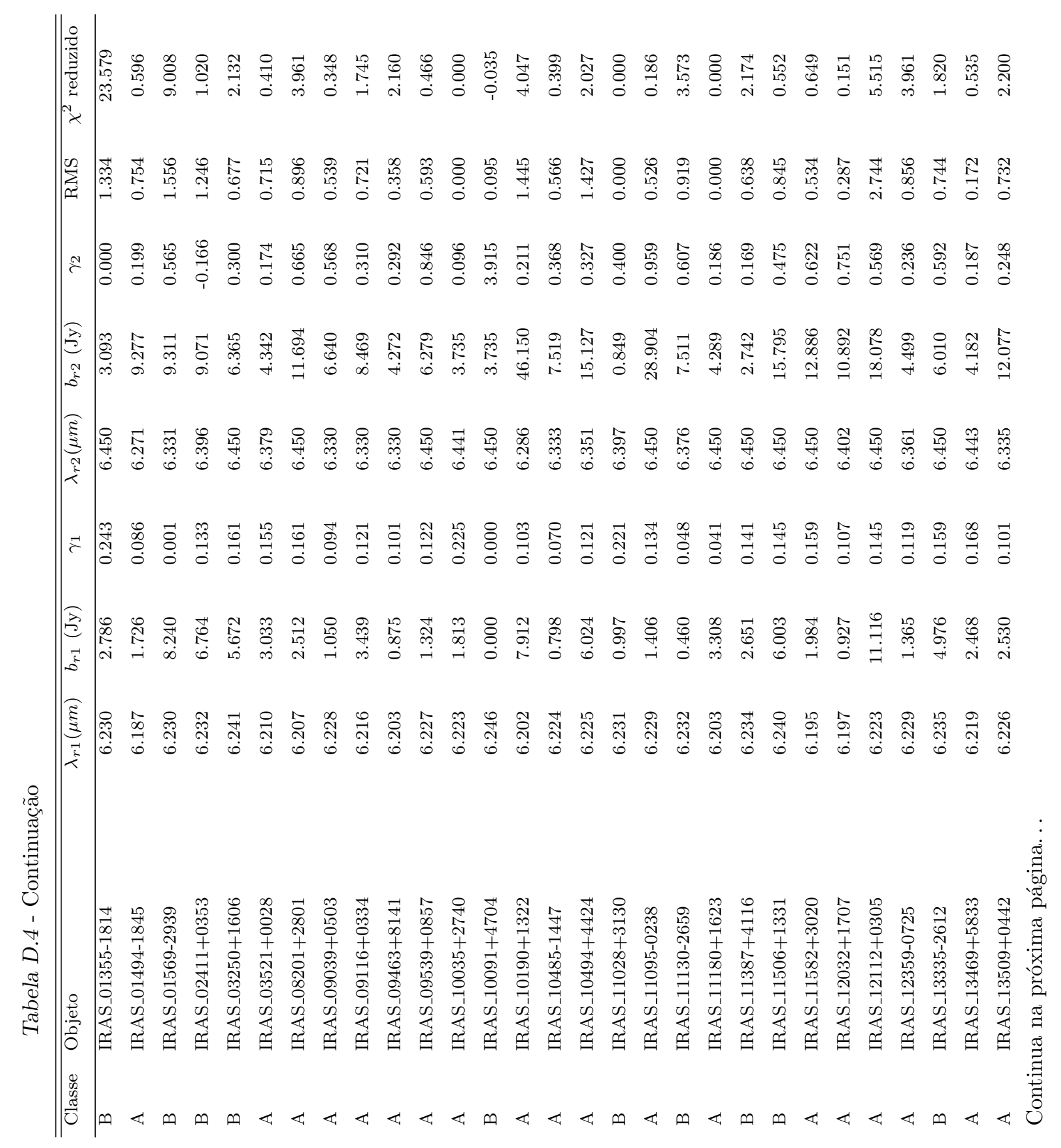




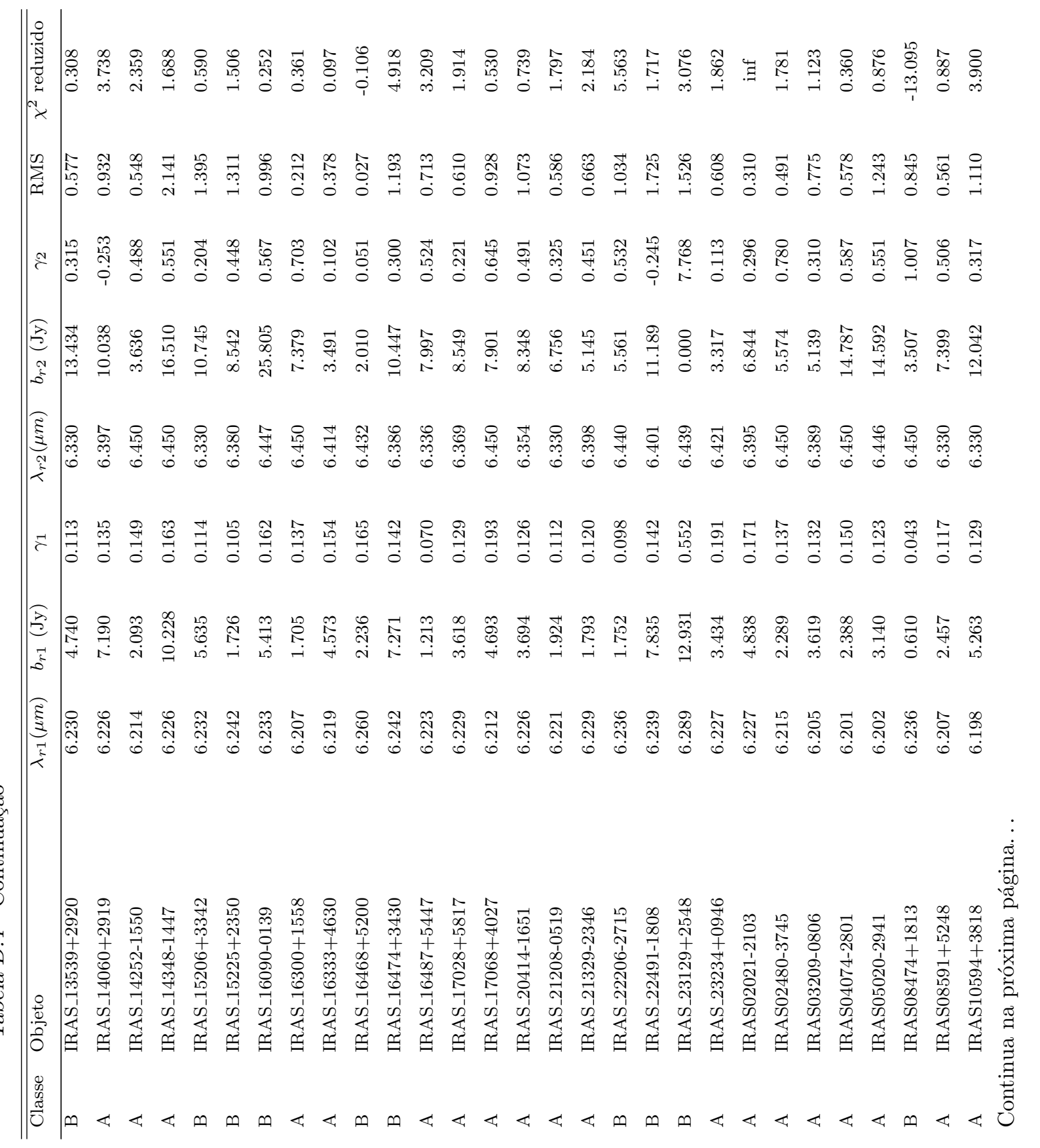




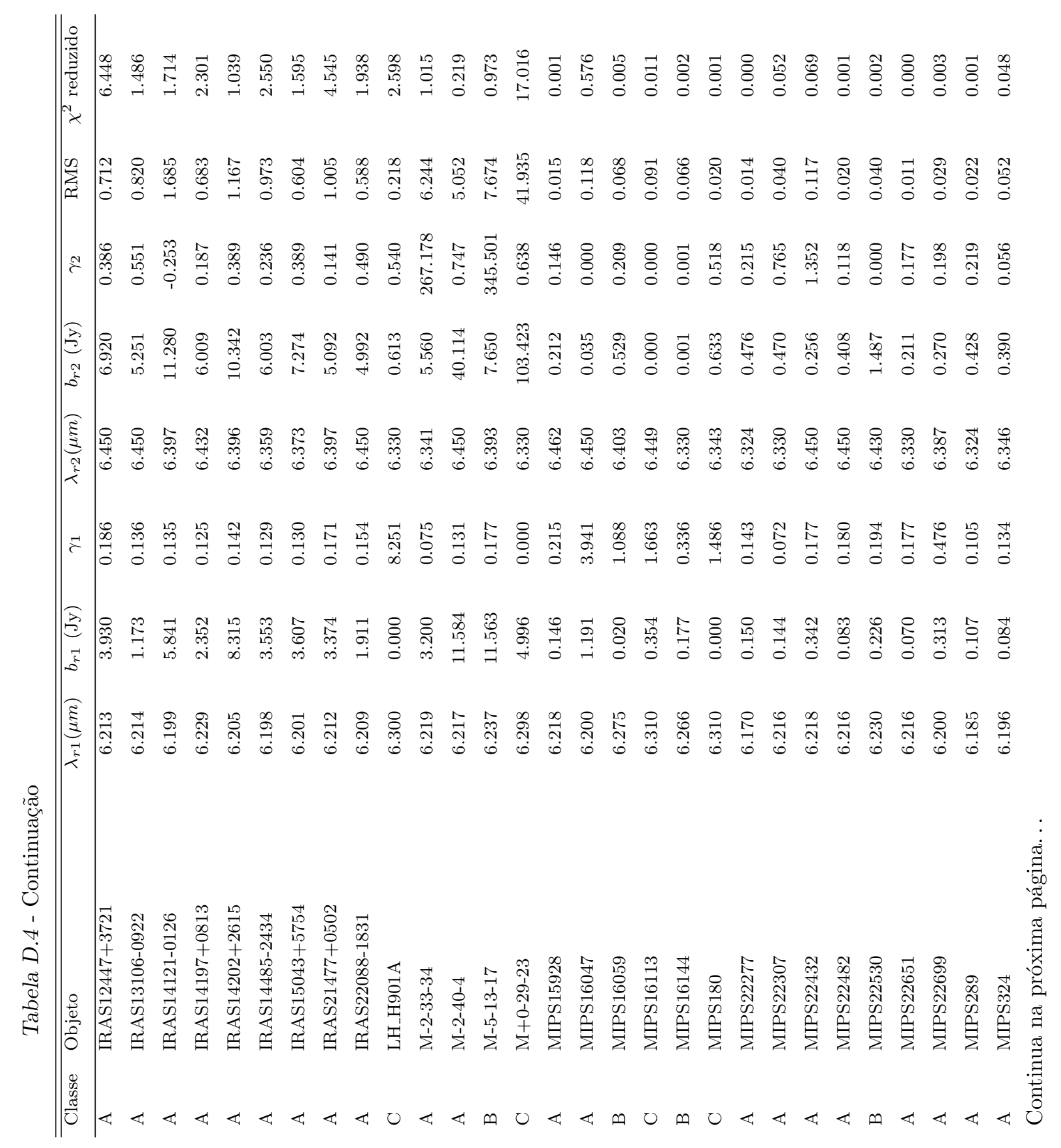




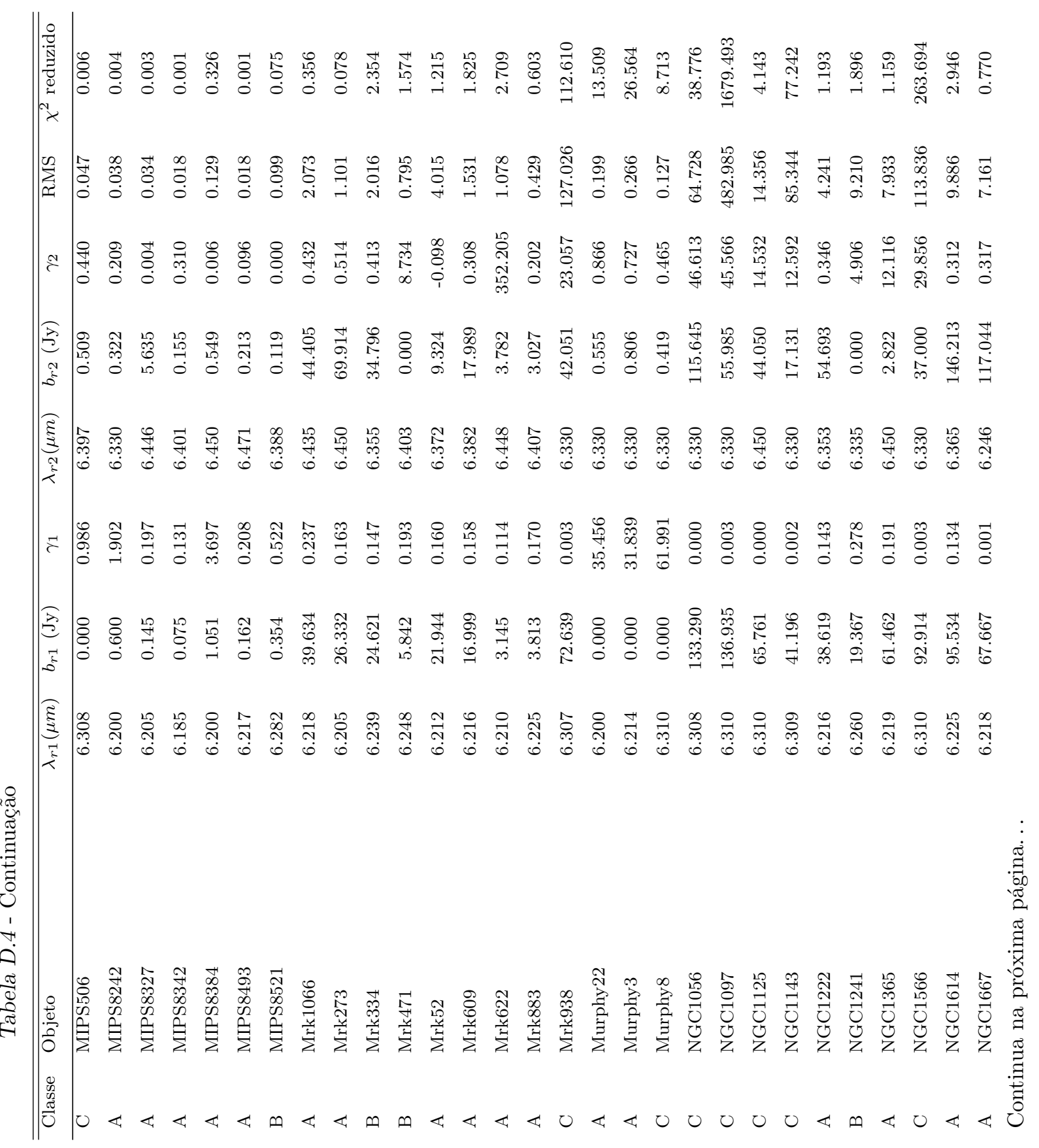




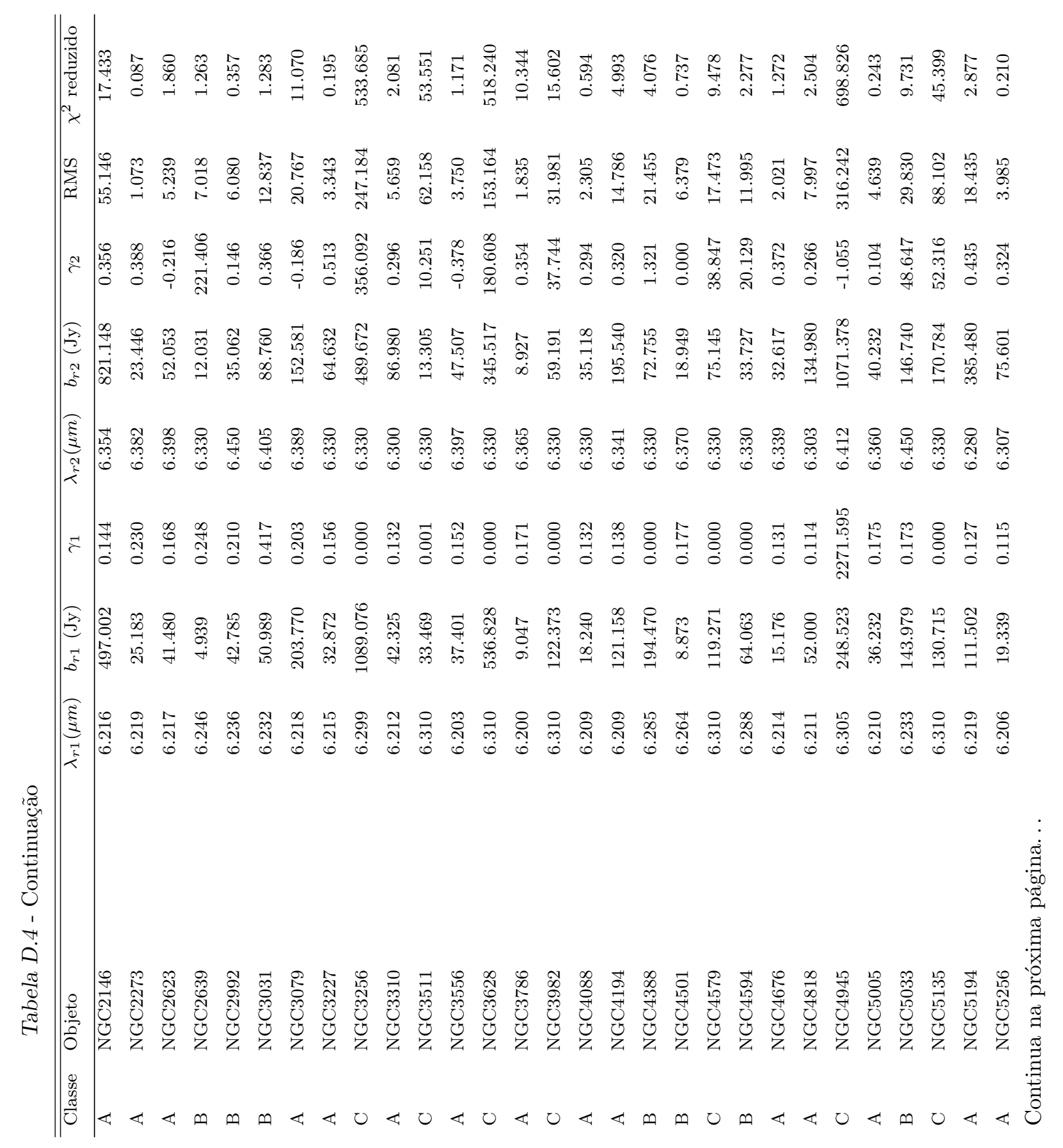




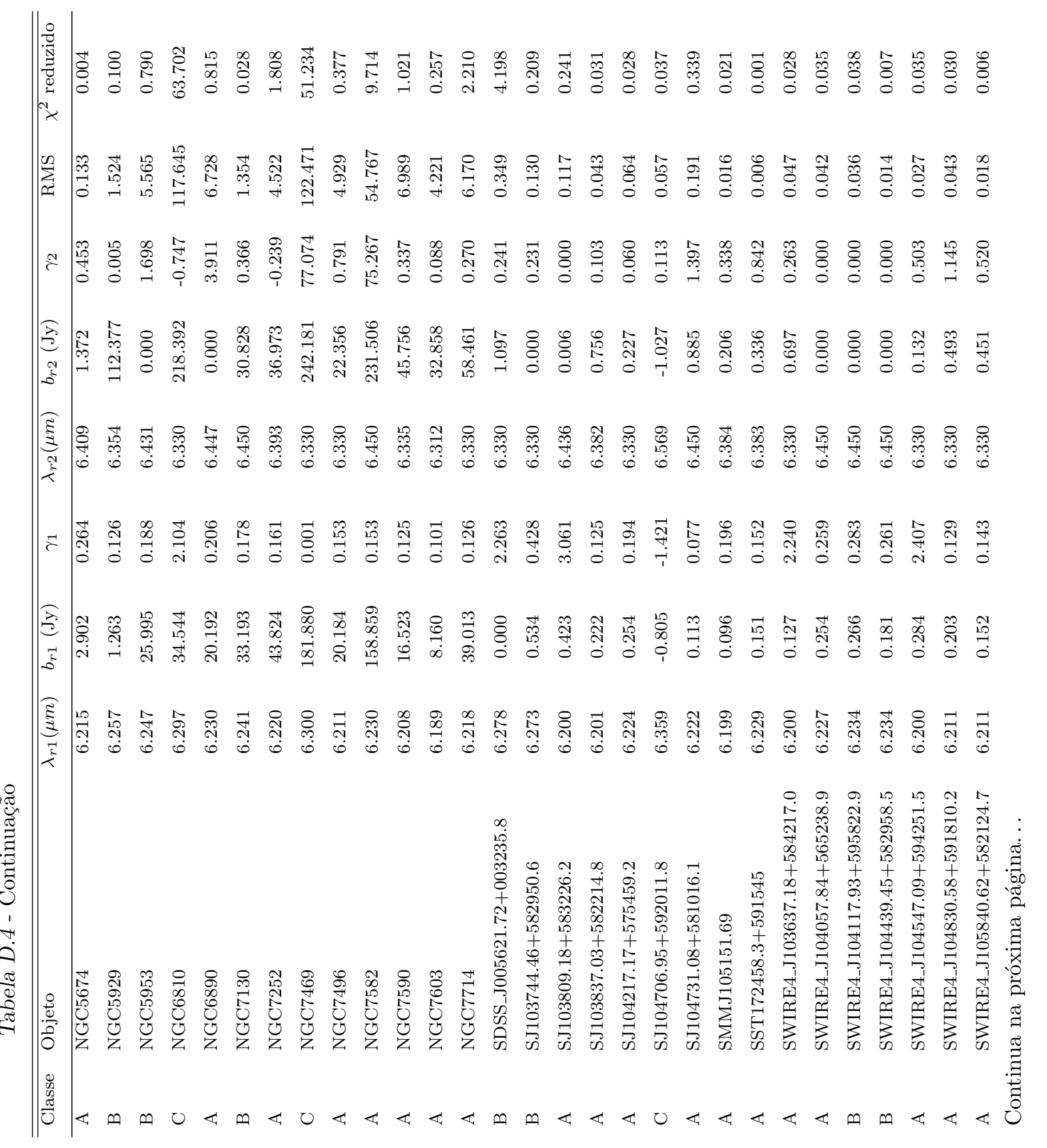




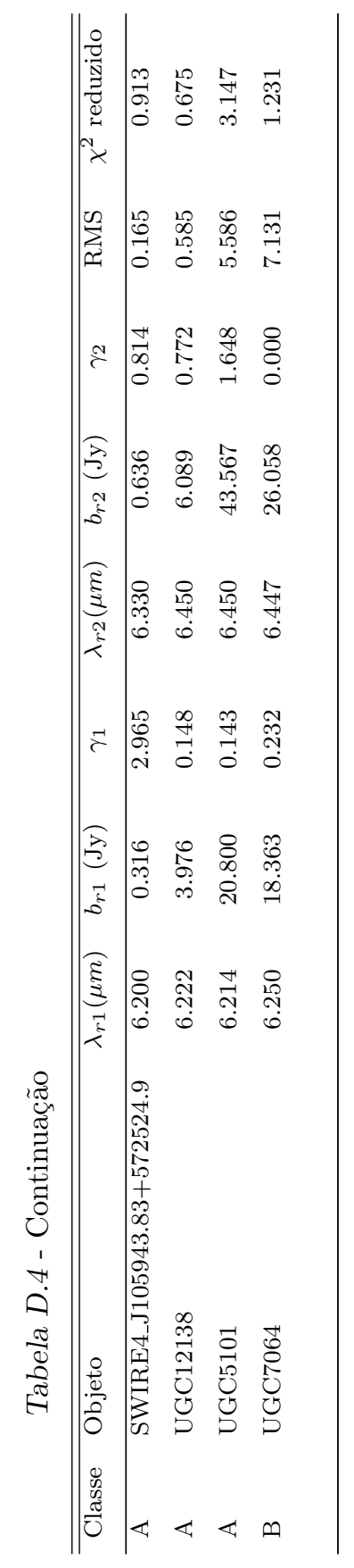


Tabela D.5 - Resultados das galáxias que apresentaram os melhores ajustes. Aquelas que não contêm as incertezas da posição central do pico foram ajustadas com minimize, as demais são do curve_fit.

\begin{tabular}{|c|c|c|c|c|c|}
\hline Tipo & Classe & Objeto & $\lambda_{r}(\mu m)$ & Erro $\lambda_{r}(\mu m)$ & Redshift \\
\hline AGN2 & $\mathrm{B}$ & SJ104706.95+592011.8 & 6.244 & 0.008 & 1.04 \\
\hline FR-1 & $\mathrm{A}$ & 3C31 & 6.228 & 0.005 & 0.017 \\
\hline IRgal & $\mathrm{B}$ & MIPS16047 & 6.252 & 0.009 & 0.52319 \\
\hline IRgal & $\mathrm{A}$ & MIPS22307 & 6.216 & - & 0.7 \\
\hline IRgal & $\mathrm{A}$ & MIPS22432 & 6.222 & 0.005 & 1.59 \\
\hline LINER & $\mathrm{B}$ & AGN15 & 6.259 & - & 0.322 \\
\hline QSO & $\mathrm{B}$ & EIRS-41 & 6.258 & 0.011 & 0.405 \\
\hline QSO2 & $\mathrm{B}$ & LH_H901A & 6.239 & 0.007 & 0.205 \\
\hline SB & $\mathrm{B}$ & EIRS-13 & 6.265 & 0.008 & 1.0913 \\
\hline SB & $\mathrm{A}$ & EIRS-14 & 6.216 & - & 0.6154 \\
\hline SB & $\mathrm{A}$ & EIRS-16 & 6.215 & 0.006 & 0.7714 \\
\hline SB & $\mathrm{B}$ & EIRS-2 & 6.23 & 0.005 & 1.1433 \\
\hline SB & $\mathrm{A}$ & EIRS-21 & 6.215 & 0.007 & 1.028 \\
\hline SB & B & EIRS-3 & 6.286 & 0.007 & 0.6761 \\
\hline SB & A & EIRS-32 & 6.227 & 0.007 & 0.6154 \\
\hline SB & A & EIRS-9 & 6.225 & - & 0.6055 \\
\hline SB & A & IC342 & 6.212 & - & 0.001 \\
\hline SB & A & Mrk52 & 6.212 & - & 0.0071 \\
\hline SB & A & NGC1222 & 6.216 & - & 0.0076 \\
\hline SB & $\mathrm{A}$ & NGC2146 & 6.216 & - & 0.0039 \\
\hline SB & $\mathrm{A}$ & NGC2623 & 6.217 & - & 0.0185 \\
\hline SB & A & NGC3256 & 6.215 & 0.002 & 0.0084 \\
\hline SB & $\mathrm{A}$ & NGC3556 & 6.203 & - & 0.0032 \\
\hline SB & A & NGC4088 & 6.209 & - & 0.0031 \\
\hline SB & A & NGC4676 & 6.214 & - & 0.0225 \\
\hline SB & $\mathrm{A}$ & NGC4818 & 6.211 & - & 0.0022 \\
\hline SB & $\mathrm{A}$ & NGC7252 & 6.22 & - & 0.0158 \\
\hline SB & $\mathrm{A}$ & SJ103837.03+582214.8 & 6.201 & - & 1.68 \\
\hline SB & $\mathrm{B}$ & SJ104217.17+575459.2 & 6.238 & 0.005 & 1.91 \\
\hline SB & $\mathrm{A}$ & SJ104731.08+581016.1 & 6.218 & 0.005 & 1.81 \\
\hline $\mathrm{SB}+\mathrm{HII}$ & $\mathrm{A}$ & NGC1614 & 6.225 & - & 0.0149 \\
\hline $\mathrm{SB}+\mathrm{HII}$ & $\mathrm{A}$ & NGC3310 & 6.212 & - & 0.0047 \\
\hline $\mathrm{SB}+\mathrm{HII}$ & $\mathrm{A}$ & NGC4194 & 6.209 & - & 0.0095 \\
\hline $\mathrm{SB}+\mathrm{HII}$ & $\mathrm{A}$ & NGC7714 & 6.218 & - & 0.009 \\
\hline SB + LINER & $\mathrm{A}$ & NGC3628 & 6.225 & 0.004 & 0.0023 \\
\hline $\mathrm{SB}+\mathrm{Sy} 1$ & $\mathrm{~A}$ & NGC1097 & 6.215 & 0.003 & 0.004 \\
\hline $\mathrm{SB}+\mathrm{Sy} 1.8$ & $\mathrm{~A}$ & NGC1365 & 6.219 & 0.003 & 0.0054 \\
\hline $\mathrm{SB}+\mathrm{Sy} 2$ & $\mathrm{~A}$ & NGC4945 & 6.224 & 0.003 & 0.0009 \\
\hline SMG & B & GN06 & 6.254 & 0.017 & 2 \\
\hline SMG & B & GN26 & 6.272 & 0.005 & 1.219 \\
\hline SMG & $\mathrm{A}$ & Murphy22 & 6.227 & 0.004 & 0.641 \\
\hline
\end{tabular}

Continua na próxima página... 
Tabela D.5 - Continuação

\begin{tabular}{|c|c|c|c|c|c|}
\hline Tipo & Classe & Objeto & $\lambda_{r}(\mu m)$ & Erro $\lambda_{r}(\mu m)$ & Redshift \\
\hline SMG & $\mathrm{B}$ & Murphy3 & 6.238 & 0.004 & 0.638 \\
\hline SMG & B & Murphy8 & 6.251 & 0.004 & 0.639 \\
\hline SMG & $\mathrm{A}$ & SMMJ105151.69 & 6.199 & - & 1.62 \\
\hline Sy1 & A & E12-G21 & 6.212 & - & 0.0328 \\
\hline Sy1 & $\mathrm{A}$ & IRAS_14348-1447 & 6.226 & - & 0.083 \\
\hline Sy1 & $\mathrm{A}$ & IRAS03209-0806 & 6.205 & - & 0.1664 \\
\hline Sy1 & A & M-2-33-34 & 6.215 & 0.006 & 0.015 \\
\hline Sy1 & $\mathrm{A}$ & MIPS324 & 6.196 & - & 0.96 \\
\hline Sy1 & $\mathrm{A}$ & NGC2273 & 6.219 & - & 0.006 \\
\hline Sy1 & B & NGC3511 & 6.244 & 0.004 & 0.004 \\
\hline Sy1 & $\mathrm{A}$ & NGC4579 & 6.201 & 0.005 & 0.005 \\
\hline Sy1 & A & SST172458.3+591545 & 6.229 & - & 0.494 \\
\hline Sy1 & $\mathrm{A}$ & UGC5101 & 6.214 & - & 0.039 \\
\hline Sy 1.5 & B & M-5-13-17 & 6.239 & 0.007 & 0.012 \\
\hline Sy 1.5 & $\mathrm{~A}$ & NGC1566 & 6.223 & 0.004 & 0.005 \\
\hline Sy 1.5 & A & NGC3227 & 6.215 & - & 0.004 \\
\hline Sy1.5 & $\mathrm{A}$ & NGC7469 & 6.221 & 0.003 & 0.016 \\
\hline Sy 1.8 & B & Mrk334 & 6.239 & - & 0.022 \\
\hline Sy1.8 & B & Mrk471 & 6.249 & 0.005 & 0.034 \\
\hline Sy1.8 & A & Mrk609 & 6.216 & - & 0.034 \\
\hline Sy1.8 & $\mathrm{A}$ & NGC3786 & 6.211 & 0.01 & 0.009 \\
\hline Sy 1.8 & A & NGC5033 & 6.217 & 0.002 & 0.003 \\
\hline Sy1.8 & A & UGC12138 & 6.222 & - & 0.025 \\
\hline Sy1.9 & $\mathrm{A}$ & M-2-40-4 & 6.217 & - & 0.025 \\
\hline Sy1.9 & $\mathrm{A}$ & Mrk883 & 6.225 & - & 0.038 \\
\hline Sy1.9 & B & NGC2992 & 6.236 & - & 0.008 \\
\hline Sy1.9 & B & NGC3982 & 6.238 & 0.006 & 0.004 \\
\hline Sy1.9 & $\mathrm{A}$ & NGC5674 & 6.215 & - & 0.025 \\
\hline Sy1.9 & B & NGC6890 & 6.231 & 0.009 & 0.008 \\
\hline Sy1.9 & B & NGC7130 & 6.241 & - & 0.016 \\
\hline Sy1.9 & B & UGC7064 & 6.24 & 0.011 & 0.025 \\
\hline Sy2 & A & IRAS_09539+0857 & 6.227 & - & 0.129 \\
\hline Sy2 & B & IRAS_15206+3342 & 6.232 & - & 0.125 \\
\hline Sy 2 & $\mathrm{~A}$ & IRAS_16300+1558 & 6.207 & - & 0.242 \\
\hline Sy2 & A & $M+0-29-23$ & 6.227 & 0.003 & 0.025 \\
\hline Sy2 & $\mathrm{A}$ & Mrk1066 & 6.218 & - & 0.012 \\
\hline Sy2 & $\mathrm{A}$ & Mrk273 & 6.205 & - & 0.038 \\
\hline Sy2 & $\mathrm{A}$ & Mrk622 & 6.21 & 0.004 & 0.023 \\
\hline Sy2 & $\mathrm{A}$ & Mrk938 & 6.217 & 0.002 & 0.02 \\
\hline Sy2 & B & NGC1056 & 6.232 & 0.006 & 0.005 \\
\hline Sy2 & $\mathrm{A}$ & NGC1125 & 6.218 & 0.003 & 0.011 \\
\hline Sy 2 & B & NGC1143 & 6.239 & 0.006 & 0.029 \\
\hline Sy2 & $\mathrm{A}$ & NGC1667 & 6.218 & - & 0.015 \\
\hline Sy2 & A & NGC3079 & 6.218 & - & 0.004 \\
\hline Sy2 & $\mathrm{B}$ & NGC4388 & 6.233 & 0.006 & 0.008 \\
\hline
\end{tabular}

Continua na próxima página... 
Tabela D.5 - Continuação

\begin{tabular}{|c|c|c|c|c|c|}
\hline Tipo & Classe & Objeto & $\lambda_{r}(\mu m)$ & Erro $\lambda_{r}(\mu m)$ & Redshift \\
\hline Sy2 & A & NGC5005 & 6.21 & - & 0.003 \\
\hline Sy2 & $\mathrm{A}$ & NGC5135 & 6.227 & 0.004 & 0.014 \\
\hline Sy2 & A & NGC5194 & 6.219 & - & 0.002 \\
\hline Sy2 & $\mathrm{A}$ & NGC5256 & 6.206 & - & 0.028 \\
\hline Sy2 & B & NGC5953 & 6.239 & 0.005 & 0.007 \\
\hline Sy2 & A & NGC6810 & 6.22 & 0.003 & 0.007 \\
\hline Sy2 & A & NGC7496 & 6.211 & - & 0.006 \\
\hline Sy2 & A & NGC7582 & 6.222 & 0.003 & 0.005 \\
\hline Sy2 & A & NGC7590 & 6.208 & - & 0.005 \\
\hline ULIRG & A & Arp220 & 6.226 & 0.004 & 0.018 \\
\hline ULIRG & A & IRAS_01494-1845 & 6.187 & - & 0.158 \\
\hline ULIRG & A & IRAS02021-2103 & 6.227 & - & 0.116 \\
\hline ULIRG & A & IRAS02480-3745 & 6.215 & - & 0.165 \\
\hline ULIRG & A & IRAS04074-2801 & 6.201 & - & 0.153 \\
\hline ULIRG & A & IRAS05020-2941 & 6.202 & - & 0.154 \\
\hline ULIRG & A & IRAS08591+5248 & 6.207 & - & 0.158 \\
\hline ULIRG & A & IRAS10594+3818 & 6.198 & - & 0.158 \\
\hline ULIRG & A & IRAS12447+3721 & 6.213 & - & 0.158 \\
\hline ULIRG & A & IRAS13106-0922 & 6.229 & 0.01 & 0.1745 \\
\hline ULIRG & A & IRAS14121-0126 & 6.199 & - & 0.1502 \\
\hline ULIRG & A & IRAS14197+0813 & 6.229 & - & 0.131 \\
\hline ULIRG & A & IRAS14202+2615 & 6.205 & - & 0.1587 \\
\hline ULIRG & A & IRAS14485-2434 & 6.198 & - & 0.148 \\
\hline ULIRG & A & IRAS15043+5754 & 6.201 & - & 0.1505 \\
\hline ULIRG & A & IRAS21477+0502 & 6.212 & - & 0.171 \\
\hline ULIRG & A & IRAS22088-1831 & 6.209 & - & 0.1702 \\
\hline ULIRG & A & MIPS15928 & 6.218 & - & 1.52 \\
\hline ULIRG & $\mathrm{C}$ & MIPS180 & 6.337 & 0.008 & 2.47 \\
\hline ULIRG & A & MIPS22277 & 6.17 & - & 1.77 \\
\hline ULIRG & A & MIPS22482 & 6.216 & - & 1.84 \\
\hline ULIRG & A & MIPS22530 & 6.23 & 0.002 & 1.951 \\
\hline ULIRG & $\mathrm{A}$ & MIPS22651 & 6.216 & - & 1.73 \\
\hline ULIRG & A & MIPS289 & 6.185 & - & 1.86 \\
\hline ULIRG & $\mathrm{C}$ & MIPS8242 & 6.305 & 0.002 & 2.45 \\
\hline ULIRG & A & MIPS8327 & 6.205 & 0.003 & 2.443 \\
\hline ULIRG & A & MIPS8342 & 6.185 & - & 1.57 \\
\hline ULIRG & A & MIPS8493 & 6.217 & - & 1.8 \\
\hline ULIRG & $\mathrm{C}$ & SWIRE4_J103637.18+584217.0 & 6.313 & 0.003 & 0.97 \\
\hline ULIRG & A & SWIRE4_J104057.84+565238.9 & 6.225 & 0.003 & 0.93 \\
\hline ULIRG & B & SWIRE4_J104117.93+595822.9 & 6.232 & 0.004 & 0.65 \\
\hline ULIRG & $\mathrm{B}$ & SWIRE4_J104439.45+582958.5 & 6.233 & 0.003 & 0.68 \\
\hline ULIRG & $\mathrm{B}$ & SWIRE4_J104547.09+594251.5 & 6.266 & 0.006 & 0.39 \\
\hline ULIRG & A & SWIRE4_J104830.58+591810.2 & 6.211 & - & 0.94 \\
\hline ULIRG & A & SWIRE4_J105840.62+582124.7 & 6.211 & - & 0.89 \\
\hline ULIRG & $\mathrm{A}$ & SWIRE4_J105943.83+572524.9 & 6.204 & 0.004 & 0.8 \\
\hline
\end{tabular}

Continua na próxima página... 
Tabela D.5 - Continuação

\begin{tabular}{|c|c|c|c|c|c|}
\hline Tipo & Classe & Objeto & $\lambda_{r}(\mu m)$ & Erro $\lambda_{r}(\mu m)$ & Redshift \\
\hline ULIRG + HII & $\mathrm{A}$ & IRAS_00456-2904 & 6.23 & - & 0.11 \\
\hline ULIRG + HII & $\mathrm{A}$ & IRAS_01199-2307 & 6.217 & - & 0.156 \\
\hline ULIRG + HII & B & IRAS_01355-1814 & 6.242 & 0.011 & 0.192 \\
\hline ULIRG + HII & B & IRAS_02411+0353 & 6.232 & - & 0.144 \\
\hline ULIRG + HII & A & IRAS_08201+2801 & 6.207 & - & 0.168 \\
\hline ULIRG + HII & A & IRAS_10190+1322 & 6.202 & - & 0.077 \\
\hline ULIRG + HII & B & IRAS_11506+1331 & 6.24 & - & 0.127 \\
\hline ULIRG + HII & A & IRAS_13469+5833 & 6.219 & - & 0.158 \\
\hline ULIRG + HII & A & IRAS_13509+0442 & 6.226 & - & 0.136 \\
\hline ULIRG + HII & B & IRAS_13539+2920 & 6.23 & - & 0.108 \\
\hline ULIRG + HII & A & IRAS_14060+2919 & 6.226 & - & 0.117 \\
\hline ULIRG + HII & B & IRAS_16474+3430 & 6.242 & - & 0.111 \\
\hline ULIRG + HII & A & IRAS_17068+4027 & 6.212 & - & 0.179 \\
\hline $\mathrm{ULIRG}+\mathrm{HII}$ & A & IRAS_20414-1651 & 6.226 & - & 0.086 \\
\hline ULIRG + HII & A & IRAS_21208-0519 & 6.221 & - & 0.13 \\
\hline ULIRG + HII & B & IRAS_22206-2715 & 6.236 & - & 0.132 \\
\hline ULIRG + HII & B & IRAS_22491-1808 & 6.239 & - & 0.076 \\
\hline ULIRG + LINER & B & IRAS_03250+1606 & 6.241 & - & 0.129 \\
\hline ULIRG + LINER & A & IRAS_03521+0028 & 6.21 & - & 0.152 \\
\hline ULIRG + LINER & A & IRAS_09039+0503 & 6.228 & - & 0.125 \\
\hline ULIRG + LINER & A & IRAS_09116+0334 & 6.216 & - & 0.146 \\
\hline ULIRG + LINER & A & IRAS_09463+8141 & 6.203 & - & 0.156 \\
\hline ULIRG + LINER & A & IRAS_10485-1447 & 6.224 & - & 0.133 \\
\hline ULIRG + LINER & A & IRAS_10494+4424 & 6.225 & - & 0.092 \\
\hline ULIRG + LINER & B & IRAS_11130-2659 & 6.232 & - & 0.136 \\
\hline ULIRG + LINER & A & IRAS_11582+3020 & 6.195 & - & 0.223 \\
\hline ULIRG + LINER & A & IRAS_12032+1707 & 6.197 & - & 0.217 \\
\hline ULIRG + LINER & A & IRAS_12112+0305 & 6.223 & - & 0.073 \\
\hline ULIRG + LINER & A & IRAS_12359-0725 & 6.229 & - & 0.138 \\
\hline ULIRG + LINER & B & IRAS_13335-2612 & 6.235 & - & 0.125 \\
\hline ULIRG + LINER & $\mathrm{A}$ & IRAS_14252-1550 & 6.2 & - & 0.149 \\
\hline ULIRG + LINER & B & IRAS_16090-0139 & 6.233 & - & 0.134 \\
\hline ULIRG + LINER & A & IRAS_16333+4630 & 6.219 & - & 0.191 \\
\hline ULIRG + LINER & A & IRAS_16487+5447 & 6.223 & - & 0.104 \\
\hline ULIRG + LINER & A & IRAS_17028+5817 & 6.229 & - & 0.106 \\
\hline ULIRG + LINER & A & IRAS_21329-2346 & 6.229 & - & 0.125 \\
\hline ULIRG + LINER & B & IRAS_23129+2548 & 6.232 & 0.007 & 0.179 \\
\hline ULIRG + LINER & $\mathrm{A}$ & IRAS_23234+0946 & 6.227 & - & 0.128 \\
\hline
\end{tabular}

